\title{
The Microstructure and Rheology of Complex Fluids Containing Na-caseinate
}

By

Hui Lin Tan

\author{
A thesis \\ submitted to the Victoria University of Wellington \\ in fulfilment of the requirements for the degree of \\ Doctor of Philosophy \\ in Chemistry
}


Parts of the content of this thesis have been published in the following article

Tan, Hui Lin; McGrath, Kathryn M. The microstructural and rheological properties of Nacaseinate dispersions. Journal of Colloid and Interface Science (2010), 342(2), 399-406.

Tan, Hui Lin; Feindel, Kirk W.; McGrath, Kathryn M. Shear banding in concentrated Nacaseinate emulsions. Soft Matter (2010), 6(15), 3643-3653. 


\section{Abstract}

Emulsions are widely utilised in commercial environments, such as in the food and cosmetic industries. In their simplest form, emulsions are a system consisting of two immiscible liquids in the presence of emulsifiers. To form an emulsion, an input of energy is required. In this thesis, Na-caseinate was used as the emulsifier and three systems were studied: soybean oil/Na-caseinate/water, palm oil/Na-caseinate/water and tetradecane/Nacaseinate/water.

Four main techniques were used to characterise the stabilised emulsions: laser diffraction particle sizing, PGSTE-NMR, rheology and cryo-SEM.

Emulsion systems are extremely complex making control and predictability over their phase behaviour practically difficult. This is because the required overall characteristics of these colloids are strongly dependent on both the energy of formulation and the choice of an appropriate combination of emulsifier, dispersed phase and continuous phase. A full understanding of the microstructure, stability and physicochemical properties of caseinatestabilised emulsions has as yet not been achieved. For example, how does caseinate selfassembly control emulsion stability? How do concentrated caseinate-based emulsions differ from dilute ones and how do the different oils (food grade oils vs. straight chain hydrocarbon) affect the formation of emulsions? The aim of this PhD programme was to obtain data to allow a better fundamental understanding of the underlying parameters defining emulsion behaviour to be obtained.

As a prelude to investigating concentrated Na-caseinate emulsions, the self-assembly of casein in buffer solution was studied. The influence of Na-caseinate concentration on water self-diffusion can be explained by obstruction and tortuosity effects arising due to the presence of casein aggregates. Unrestricted diffusion of casein aggregates was measured for all concentrations at short observation times only ( $20 \mathrm{~ms})$. Despite a near uniform size distribution on increasing Na-caseinate concentration the measured unrestricted diffusion coefficient is a decreasing function of Na-caseinate concentration. Enhanced packing interactions between the aggregates and the slight augmentation of the large tail in the size distribution are the main reasons for this observed decrease. On increasing the experimental observation times restricted diffusion of the aggregates is measured. 
The rheological response of the dispersions was also investigated. The stress evolution of the sample with time exhibits three distinct behaviours, irrespective of Na-caseinate concentration and applied shear rate: a spontaneous increase of stress at very short times $(<1$ s), followed by a plateau with fine structure superposed and lastly a sharp increase in stress attributed to irreversible gelation. The latter occurs at significantly reduced times on increasing Na-caseinate concentration. Linear rheology measurements indicate that the dispersions are predominantly locally liquid-like while being macroscopically in a gelled state.

For all systems investigated, oil-in-water emulsions are formed. The emulsion phase diagrams for the soybean oil, palm oil/Na-caseinate/water systems are generally more similar to each other than to the emulsion phase diagram of the tetradecane/Na-caseinate/water system. Domains are distinguished by both their macroscopic stability and their microstructure. At low Na-caseinate concentration, bridging between oil droplets occurs due to insufficient emulsifier coverage. Upon increasing protein concentration, full coverage of the oil droplet surfaces is achieved, emulsion stability is enhanced and individual droplets can be visualised. Further incrementing protein concentration reduces emulsion stability to its lowest levels due to the inducement of depletion flocculation. The final emulsion domain, occurring at the highest Na-caseinate and oil concentrations is the most stable. Here the aqueous continuous phase is comprised of a three-dimensional self-assembled protein network.

Irrespective of the type of oils and the oil and Na-caseinate concentrations, the particle size distributions of the three systems remain very similar to each other. The mean droplet diameters of the bimodal distributions are $\sim 0.12$ and $\sim 1.3 \mu \mathrm{m}$. The variation in macroscopic stability is predominantly determined by the Na-caseinate content, i.e., the extent of packing of the oil droplets, the state of the interfacial structure and the self-assembly of Na-caseinate as opposed to the oil volume fraction. Cryo-SEM micrographs reveal the difference in the microstructure of the emulsions which controls and defines the rates of phase separation. Water diffusion critically depends on the microstructure and tortuosity in addition to obstruction and/or hydration effects, which dominate the systems on increasing the oil and/or Na-caseinate content. The extracted distribution of diffusion coefficients correlates to different types of diffusion. In particular, at a fixed Na-caseinate concentration by incrementing the oil concentration, the relative intensity of the oil diffusion peak that is due to pure restricted diffusion increases, because of the already enhanced packing of the oil 
droplets and hence the oil droplets encounter each other on average more frequently.

The response of each of the different microstructures on application of shear was also examined. The form of the flow curves was not markedly altered. Competition between ageing and rejuvenation is believed to be the origin of the time evolution of the stress response, though the underlying microstructure changes are not evidenced in the flow curves. At low shear rates, all emulsions exhibit shear-thinning behaviour. At higher shear rates, a stress plateau, indicative of shear banding, is reflected in the flow curves before a Newtonian response is achieved. The results of oscillatory rheology show the emulsions formed are predominantly solid-like. The viscoelastic behaviour is Na-caseinate concentration dependence mirroring a difference in the microstructure between the emulsions and an enhancement in the viscosity. 


\section{Acknowledgements}

First and foremost, I am heartily thankful to my supervisor, Assoc. Prof. Kathryn McGrath, whose encouragement and guidance from the initial to the final level enabled me to develop an understanding on emulsions. She has supported me throughout my thesis with her patience and knowledge whilst allowing me to work in my own way. Without her this thesis would not have been completed.

A special thanks goes to Prof. Paul Callaghan for his kind support with regard to the NMR instrument. I would like to also thank Allan Raudsepp, Antoine Lutti, Bradley Douglass, Kirk Feindel, Simon Rogers and Mark Hunter for giving me a helping hand in the NMR lab and for their valuable discussions.

In the various laboratories, I have been aided in running the equipment. I am grateful to David Flynn, who helped tutor me in the techniques necessary to run scanning electron microscopy and Alan Rennie and Manu Pouajen-Blakiston for their ingenious sample holder construction. As well as ordering chemicals for me, Sally Wisheart and Lisa Meredith have also without fail provided a friendly smile and a hello every time we met.

In my daily work I have been blessed with helpful and cheerful group members: Nelly Bulgarelli, Conrad Lendrum, Benjamin Matthewson, Ben Murray, Sheinach Dunn, Nick Monahan, Ash Dangerfield, Agathe Fournier, Natasha Munro, Riyad Mucadam, Nguyen Nguyen and Catherine Davis. You will all be remembered. Thank you also to Ying Tang who has been a good companion over the last couple of years.

Special thanks to Emeritus Prof. Roger Hopkins and Ruth who always cared about my progress and have offered much advice and assistance during my stay in New Zealand. I owe my deepest gratitude to Siong Sin Wong for everything he has done for me and to my mum for providing a home in which to complete my writing up.

Lastly, I offer my regards and wishes to all of those who supported me in any respect during the completion of the project.

Hui Lin Tan

9 June 2010 


\section{Table of Contents}

1 Introduction 1

1.1 Casein Proteins 1

1.1.1 Casein Micelle Models 5

$\begin{array}{ll}\text { 1.1.2 Aggregation of Commercial Casein } & 7\end{array}$

1.2 Emulsions 9

1.3 Stability of Emulsion 11

$\begin{array}{lll}\text { 1.3.1 Gibbs Elasticity } & 11\end{array}$

$\begin{array}{lll}\text { 1.3.2 Gaussian Curvature } & 12\end{array}$

$\begin{array}{ll}\text { 1.3.3 Bending Energy } & 14\end{array}$

$\begin{array}{lll}\text { 1.3.4 Laplace Pressure } & 15\end{array}$

1.4 Casein-stabilised Emulsions 15

1.5 References 19

2 Experimental $\quad 27$

2.1 Materials 27

2.2 Emulsion Characterisation 28

2.2.1 Laser Diffraction Particle Sizing 28

2.2.1.1 The Equivalent Sphere, and The Mean Particle Diameter 28

$\begin{array}{lll}2.2 .1 .2 & \text { Light Scattering } 30\end{array}$

2.2.1.3 Mie Theory 31

2.2.1.4 Experimental Parameters 32

2.2.2 Nuclear Magnetic Resonance (NMR) 32

2.2.2.1 Nuclear Spin and Zeeman Interaction 32

2.2.2.2 Pulses and Signal Detection 34

2.2.2.3 Relaxation Measurements 36

2.2.2.3.1 Saturation Recovery 36

2.2.2.3.2 Carr-Purcell-Meiboom-Gill (CPMG) 37

2.2.2.4 Pulsed Gradient Stimulated Echo (PGSTE) NMR 38 
2.2.2.4.1 Experimental Parameters

2.2.2.5 Inverse Laplace Transformation

2.2.2.5.1 Regularisation

2.2.2.6 Imaging

2.2.2.7 NMR Velocimetry

2.2.2.7.1 Experimental Parameters

2.2.3 Rheology 46

2.2.3.1 Elastic and Viscous, Newtonian and non-Newtonian 46

2.2.3.2 Geometries 48

2.2.3.2.1 Cone-and-Plate 48

2.2.3.2.2 Concentric-cylinder Couette

2.2.3.3 Experimental Parameters

2.2.3.4 Linear Rheology

2.2.3.4.1 Experimental Parameters

2.2.4 Cryo-Scanning Electron Microscopy (Cryo-SEM)

2.2.4.1 Resolution and Depth of Field 55

2.2.4.2 Experimental Parameters 56

2.3 References

3 Na-caseinate Dispersions

3.1 Introduction

3.2 Results 60

3.2.1 Laser Diffraction Particle Sizing 61

3.2.2 PGSTE-NMR 61

3.2.3 Rheology 65

3.3 Discussion 72

3.3.1 PGSTE-NMR 72

3.3.2 Rheology 77

3.4 Conclusions 79

3.5 References 
4.2 Results 85

$\begin{array}{lll}4.3 & \text { Discussion } & 94\end{array}$

$\begin{array}{lll}4.4 & \text { Conclusions } & 101\end{array}$

$\begin{array}{llr}4.5 & \text { References } & 102\end{array}$

5 Soybean OilEmulsions 106

$\begin{array}{lll}5.1 & \text { Introduction } & 106\end{array}$

$\begin{array}{lll}5.2 & \text { Results } & 106\end{array}$

$\begin{array}{lll}\text { 5.2.1 Laser Diffraction Particle Sizing } & 107\end{array}$

$\begin{array}{lll}\text { 5.2.2 PGSTE-NMR } & 110\end{array}$

$\begin{array}{lll}5.2 .3 \text { Rheology } & 119\end{array}$

$\begin{array}{lll}\text { 5.2.4 Cryo-SEM } & 121\end{array}$

$\begin{array}{lll}5.3 & \text { Discussion } & 125\end{array}$

5.3.1 Laser Diffraction Particle Sizing 125

$\begin{array}{lll}\text { 5.3.2 } & \text { PGSTE-NMR } & 128\end{array}$

$\begin{array}{lll}\text { 5.3.3 Rheology } & 135\end{array}$

$\begin{array}{lll}\text { 5.3.4 Cryo-SEM } & 137\end{array}$

5.4 Conclusions 138

$\begin{array}{lll}5.5 & \text { References } & 140\end{array}$

6 Shear Banding 144

6.1 Introduction 144

$\begin{array}{lll}6.2 & \text { Results } & 146\end{array}$

$\begin{array}{lll}\text { 6.2.1 Rheology } & 146\end{array}$

6.2.2 NMR Velocimetry 153

$\begin{array}{lll}6.3 & \text { Discussion } & 156\end{array}$

$\begin{array}{lll}6.4 & \text { Conclusions } & 159\end{array}$

6.5 References 161

7 Palm OilEmulsions 164

$\begin{array}{lll}7.1 & \text { Introduction } & 164\end{array}$

$\begin{array}{lll}7.2 & \text { Results } & 165\end{array}$ 
7.2.1 Laser Diffraction Particle Sizing

7.2.2 PGSTE-NMR

7.2.3 Rheology

7.2.4 Cryo-SEM

7.3 Discussion

7.3.1 PGSTE-NMR

7.3.2 Rheology

7.3.3 Cryo-SEM

7.4 Conclusions

7.5 References

8 Tetradecane Emulsions

$\begin{array}{lll}\text { 8.1 Introduction } & 186\end{array}$

$\begin{array}{lll}8.2 & \text { Results } & 187\end{array}$

$\begin{array}{lll}\text { 8.2.1 } & \text { Laser Diffraction Particle Sizing } & 187\end{array}$

$\begin{array}{lll}\text { 8.2.2 PGSTE-NMR } & 189\end{array}$

$\begin{array}{lll}\text { 8.2.3 Rheology } & 192\end{array}$

8.2.4 Cryo-SEM and Confocal Microscopy 196

$\begin{array}{lll}8.3 \text { Discussion } & 198\end{array}$

$\begin{array}{lll}\text { 8.3.1 Laser Diffraction Particle Sizing } & 198\end{array}$

$\begin{array}{lll}\text { 8.3.2 PGSTE-NMR } 200 & 200\end{array}$

$\begin{array}{lll}\text { 8.3.3 Rheology } 203 & 203\end{array}$

$\begin{array}{lll}8.4 & \text { Conclusions } 205\end{array}$

$\begin{array}{lll}8.5 & \text { References } & 206\end{array}$

9 Conclusions and Future Work 208

$\begin{array}{lll}9.1 & \text { Conclusions } & 208\end{array}$

$\begin{array}{lll}9.2 & \text { Future Work } & 210\end{array}$

$\begin{array}{lll}9.3 & \text { References } & 213\end{array}$

Appendix $\quad 214$ 


\section{A66reviations}

CCP - colloidal calcium phosphate

CPMG - Carr-Purcell-Meiboom-Gill

CRT - cathode ray tube

CYCLOPS - cyclically ordered phase sequence

DLS - dynamic light scattering

DWS - diffusing wave spectroscopy

EACN - equivalent alkane carbon number

FID - free induction decay

NNLS - non-negative least squares

$O / W$ - oil-in-water

PEEK - polyether ether ketone

PGSTE-NMR - pulsed gradient stimulated echo nuclear magnetic resonance

r.f. - radio-frequency

SAXS - small angle $x$-ray scattering

SEI - secondary electron imaging

SEM - scanning electron microscopy

SGR - soft glassy rheology

W/O - water-in-oil 


\section{Chapter 1}

\section{Introduction}

\subsection{Casein Proteins}

Proteins may be divided simplistically into two structural classes, namely those which are essentially unstructured and flexible, such as the caseins, and those which possess welldefined three-dimensional structures in solution. The casein family of proteins is the major milk protein family. Caseins, in their soluble and dispersed forms, have excellent emulsifying properties due to their good solubility, surface activity, heat resistance, and water-holding capabilities, making them important ingredients in the food industry ${ }^{1-5}$.

Na-caseinate which has been used exclusively in this $\mathrm{PhD}$ programme is composed of a soluble mixture of disordered hydrophobic caseins. It is precipitated out from milk by reducing the $\mathrm{pH}$ of milk to 4.6 followed by readjustment with sodium hydroxide $(\mathrm{NaOH})^{6,7}$. The phosphate residue positions of individual caseins, i.e., $\alpha_{\mathrm{s} 1^{-}}, \alpha_{\mathrm{s} 2^{-}}, \beta-$, and $\kappa$-casein are shown in Fig. 1.1. $\kappa$-casein is calcium-insensitive while $\alpha_{\mathrm{s} 1^{-}}, \alpha_{\mathrm{s} 2^{-}}$and $\beta$-casein are the three calcium-sensitive members. The calcium-sensitive caseins are highly phosphoserylated and the phosphoseryl residues tend to be found in two, three or four clusters. The amphipathic nature of the caseins is preserved across species (in the milk of all mammals) with each of the caseins showing their own pattern of segregation into hydrophobic and hydrophilic regions, the latter containing the phosphoseryl clusters.

The individual caseins are described as block copolymers. The rationale of such a representation comes from the fact that caseins share some aspects of solution behaviour with synthetic block copolymers. Block copolymers have been shown to form structured mesophases at sufficiently high polymer concentration ${ }^{8}$. Such behaviour is mirrored in casein self-assembly but additionally the caseins have the ability to form plastic-like structures (casein-buttons and casein-films) suggesting that they may exhibit ordered structures at high concentration. 


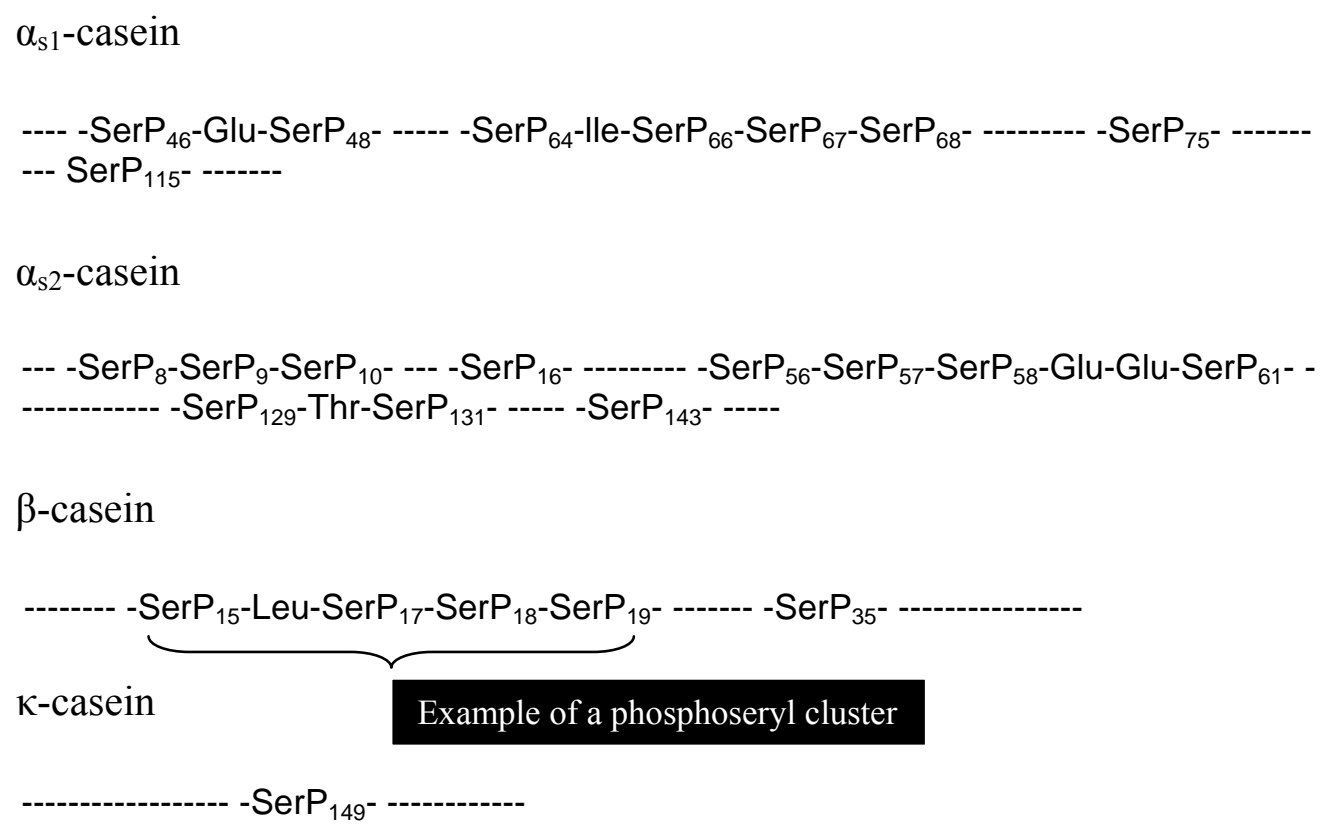

Figure 1.1: Phosphate residue positions in the bovine caseins, indicating the phosphoseryl clusters taken from 9.

The caseins exhibit strong tendencies to self-associate ${ }^{10,11}$. Using thermodynamically equivalent models, $\beta$-casein and reduced $\kappa$-casein, $\alpha_{\mathrm{s} 2}-$ and $\alpha_{\mathrm{s} 1}$-casein associate according to the equilibria:

$$
\begin{aligned}
i \beta & \rightleftarrows \beta_{i}, \\
\left(\alpha_{\mathrm{s} 2}\right)_{i}+\alpha_{\mathrm{s} 2} & \rightleftarrows\left(\alpha_{\mathrm{s} 2}\right)_{i+1}{ }^{12} \text { and } \\
\alpha_{i}+\alpha_{1} & \rightleftarrows \alpha_{i+1}{ }^{13},
\end{aligned}
$$

respectively where $i>1^{14}$. Under certain conditions, $\alpha_{\mathrm{s} 1}$-casein has been observed to form worm-like chains where the hydrophobic regions of the individual chains join end-to-end ${ }^{15}$. This is depicted in Fig. 1.2. It has also been hypothesised that $\alpha_{\mathrm{s} 1}$-casein might form a flower-like structure from petal-like loops where the two hydrophobic ends of the chains selfassociate. Much less is known about $\beta$ - and $\alpha_{\mathrm{s} 2}$-casein $^{16}$. The shape of self-associated $\beta$ - and $\alpha_{\mathrm{s} 2}$-casein have been speculated to be ellipsoid ${ }^{8,17}$ and linear (i.e., similar to $\alpha_{\mathrm{s} 1}$-casein) ${ }^{10}$, respectively.

$\alpha_{\mathrm{s} 1^{-}}$and $\beta$-casein are the two major individual caseins comprising around $75 \%$ of the total milk casein. In solution, at neutral $\mathrm{pH}$, both protein monomers adopt flexible disordered conformations with a net negative charge and a higher net charge density on the adsorbed $\alpha_{\mathrm{s} 1^{-}}$ casein layer. The caseins are highly surface active ${ }^{18}$. This argument is based on the high proportion of accessible non-polar residues in their amino-acid sequences (Fig. 1.1) ${ }^{19}$. The individual caseins have different surface properties. $\beta$-casein, in particular, has a low surface 
viscosity; 15 times lower than that of Na-caseinate ${ }^{20} . \quad \alpha_{s 1^{-}}$and $\beta$-casein are strongly amphiphilic. They contain phosphoseryl clusters of residues and a high proportion of accessible non-polar (i.e., hydrophobic) residues which implies a strong tendency to adsorb at hydrophobic surfaces ${ }^{21}$. The adsorption of these two proteins at oil/water interfaces will be discussed in the following section.

$\beta$-casein

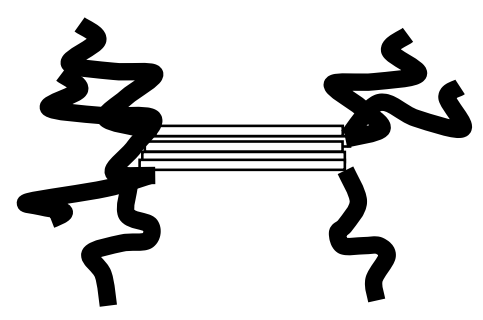

detergent-like

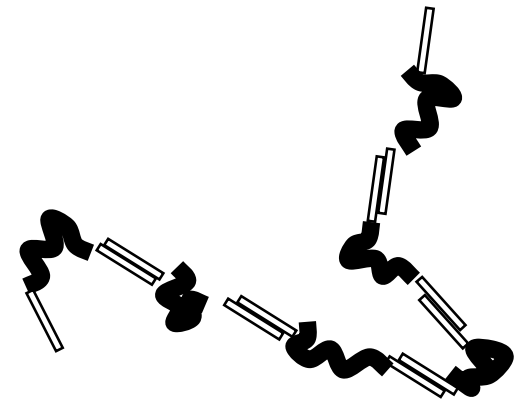

worm-like (observed) $\alpha_{\mathrm{s} 1}$-casein

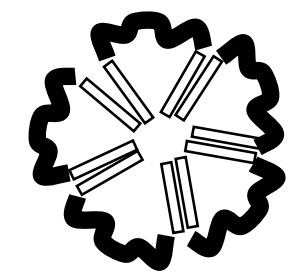

flower-like (speculative)

Figure 1.2: Structures observed and proposed for individual casein self-association taken from 22.

The simplest model to represent the adsorbed layer structure of caseins is the train-looptail model ${ }^{23,24}$. The total effective adsorbed layer thickness is mainly determined by the distribution of 'loops' and 'tails'. For a complex molecule like $\beta$-casein, the tail/loop distribution depends on the average segment density profile which is a weighted balance of wall-segment, solvent-segment and segment-segment interactions (ionic, hydrophobic, van der Waals, etc.). A sketch picture of the adsorbed $\beta$-casein molecule on a hydrophobic surface is shown in Fig. 1.3 (a). The protein exists as a highly charged tail dangling away from the surface and the remaining chain which is predominantly hydrophobic, existing as trains and small loops. In emulsions, $\beta$-casein is preferentially adsorbed at the oil/water interface formed with relatively low levels of Na-caseinate as opposed to the case that contains substantial levels of unadsorbed $\operatorname{protein}^{25-27}$.

In comparison to adsorbed $\beta$-casein, the hydrophilic/hydrophobic segment distribution in $\alpha_{\mathrm{s} 1}$-casein, shown in Fig. 1.3 (b), lies in closer association with the surface. That part lying furthest away is the highly charged, hydrophilic region. This region, which is in the middle of the chain, is separated from the two patches of hydrophobic region. These patches anchor 
the molecule firmly at the surface, forming a loop rather than an extended tail.

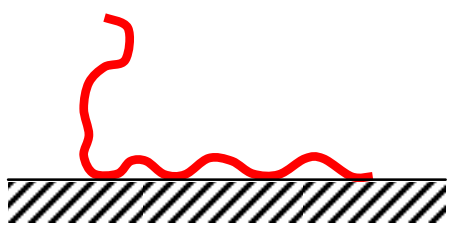

(a)

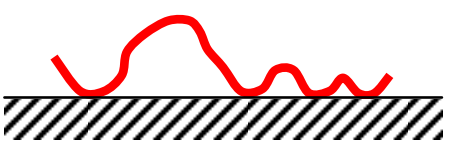

(b)

Figure 1.3: Sketch of proposed typical adsorbed configurations at a solid hydrophobic surface for (a) $\beta$-casein and (b) $\alpha_{\mathrm{s} 1}$-casein ${ }^{28}$.

Protein adsorption at oil/water interfaces has been widely discussed ${ }^{29-34}$. In gaining some insights into the properties of emulsions (presented in the following chapters), this section focuses on the conformational states of $\alpha_{\mathrm{s} 1}$ - and $\beta$-casein which define the structure of the adsorbed layers.

Using small angle x-ray scattering (SAXS) and neutron reflectance, Dickinson et al. showed that most of the mass of adsorbed $\beta$-casein is close to the interface ${ }^{35}$. However, dynamic light scattering (DLS) experiments carried out by Dalgleish et al. revealed that the hydrodynamic thickness of the adsorbed $\beta$-casein is quite large ${ }^{36}$. Collectively, this indicates that the adsorbed layer is not a multilayer because its dimensions are not altered by dissociating buffer solutions ${ }^{37}$. Nylander et al. suggested that adsorbed $\beta$-casein forms a rather tenuous, extended layer at oil/water interfaces, and this extended layer prevents further sequential adsorption of proteins ${ }^{38}$. At high protein loads, i.e., in excess of $3 \mathrm{mg} \mathrm{m}^{-2}$, multilayers can be formed ${ }^{39}$.

Dissociation of $\kappa$-casein by treatment with 2 -mercaptoethanol, either before or after adsorption, results in a thicker rather than thinner adsorbed layer ${ }^{36,40}$. Presumably the dissociation of the oligomeric protein causes a conformational change in the monomers, such that they protrude further into solution, or adsorb via different parts of their structures.

Fang et al. demonstrated that the adsorbed layer thickness of $\beta$-casein and whole casein (the natural mixture of the four caseins) in emulsions is similar ${ }^{41}$. The conformational state of the adsorbed casein is highly dependent on the ratio of protein-to-oil. At low protein-to-oil ratio, the casein must necessarily spread over a maximum area. In the presence of excess casein, there is sufficient protein to cover the oil surface without spreading. Thus, there are two conformations of the adsorbed casein at the interface, i.e., whether the molecules are spread or packed ${ }^{42}$. As the caseins are rather flexible, it is likely that they change conformation upon adsorption. 
Proteolysis may also be used to obtain information on the structures of adsorbed proteins. A particular proteolytic enzyme is used to attack the sensitive residues in the $\operatorname{protein}^{43}$. For instance, when a solution of monomeric $\beta$-casein (at $4^{\circ} \mathrm{C}$ to prevent aggregation) is incubated with trypsin, the susceptible sites, i.e., lysine and arginine residues are attacked at about the same rate ${ }^{44}$. The protein substrate is then adsorbed to an interface. Some locations in the adsorbed protein project from the surface and will be accessible while others are protected from trypsinolysis. Therefore, by determining which parts of the adsorbed protein fall into these classes, it is possible to define which parts of the molecule are on the interface.

The hydrophilic region of $\beta$-casein, containing the charged residues and all of the five phosphoseryl groups, is least likely to participate in the adsorption process. Shimuzu et al. suggested that it is the middle of the $\alpha_{\mathrm{s} 1}$-casein molecule that adsorbs to oil/water interfaces ${ }^{43}$, rather than the end as in $\beta$-casein causing the adsorbed layers of $\alpha_{\mathrm{s} 1}$-casein to be thinner than $\beta$-casein. Sketches of the adsorbed $\alpha_{\mathrm{s} 1}$ - and $\beta$-casein are shown in Fig. 1.4.

(a)
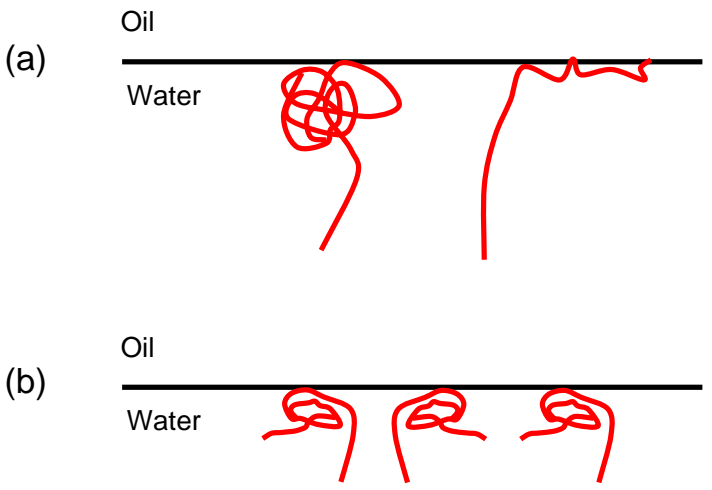

Figure 1.4: Sketches of conformations for $\beta$ - (a) and $\alpha_{\mathrm{s} 1}$-caseins (b) at oil/water interface. For $\beta$-casein, the two proposed conformations indicate most of the mass is near the interface with the hydrophilic tail protruding into the solution. For $\alpha_{\mathrm{s} 1}$-casein, the point of attachment is near the middle of the molecule and the tails protrude less ${ }^{42}$.

\subsubsection{Casein Micelle Models}

In bovine milk, the caseins exist as approximately spherical, large macro-molecular $\left(10^{8} \mathrm{Da}\right.$, $\sim 200 \mathrm{~nm}$ diameter) associates in colloidal dispersion, historically called casein micelles ${ }^{45-47}$. These native casein micelles are composed of the four casein proteins $\left(\alpha_{\mathrm{s} 1^{-}}, \alpha_{\mathrm{s} 2^{-}}, \beta-\right.$, and $\kappa-$ casein) in a ratio of $4: 1: 4: 1$ and essential nanoclusters of calcium phosphate ${ }^{48}$, called colloidal calcium phosphate (CCP), which are involved in the integrity of the micelle together with 
hydrophobic attractions.

These casein micelles are very stable, they can be frozen and even dried ${ }^{49}$. The extreme stability of casein micelles in various conditions is due to the hairy layer of hydrophilic peptides of $\kappa$-casein protruding on the surface of the micelle ${ }^{8}$. These protuberances appear to be formed from cylindrical or tubular structures, between 10 and $20 \mathrm{~nm}$ in diameter with lengths as long as $40 \mathrm{~nm}$. Bunches of collapsed $\kappa$-casein hairs linked by fixative have also been seen in electron micrographs of native casein micelles. The so-called steric stabilisation is (mainly) entropic in nature and would be absent if the hairy layer was not sufficiently dense $^{8}$.

Various models for the structure of casein micelles have been proposed over the last few decades with the progress recently reviewed by Fox ${ }^{50}$. Though with refinement and tinkering, the submicelle model described by Slattery et al. ${ }^{51,52}$ with chief elaboration by Schmidt et al. $^{53}$ remains one of the most enduring models. In this submicelle model, the caseins first aggregate via the hydrophobic interaction into subunits of 15 to 20 molecules each with a variation in the $\kappa$-casein content of these submicelles. Those rich in $\kappa$-casein congregate on the micelle surface, those submicelles poor or totally deficient in $\kappa$-casein are located in the interior of the micelle as depicted in Fig. 1.5. The elaboration of Schmidt et al. is to link these interior submicelles by colloidal calcium phosphate.
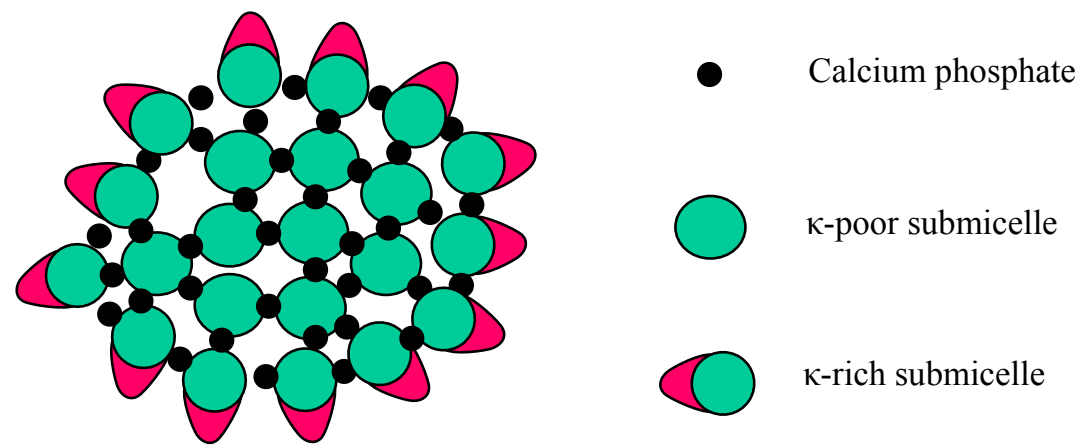

Figure 1.5: The schematic of the submicelle model of the casein micelle taken from 18.

In the later refinements of the model produced by Holt et al. ${ }^{54,55}$, the calcium phosphate is in the form of nanoclusters and the interaction sites on the caseins are the phosphoseryl clusters of the calcium-sensitive caseins (Fig. 1.1). Because $\alpha_{s^{-}}$and $\alpha_{s 2}$-caseins have more than two such clusters, they are able to cross-link the nanoclusters into extended threedimensional network structures, as illustrated in Fig. 1.6. The monofunctional $\beta$-casein closes off the facet to which it attaches and prevents further growth in that direction. $\kappa$-casein 
does not feature in this assembly process because it does not possess a phosphoseryl cluster grouping with which it could interact with the nanocluster. The micelle size, or at least network size, should be a function of the number or proportion of multifunctional casein molecules in the system. Loop formation is a random event, and therefore micelle size should also be random and occur in a range of sizes.

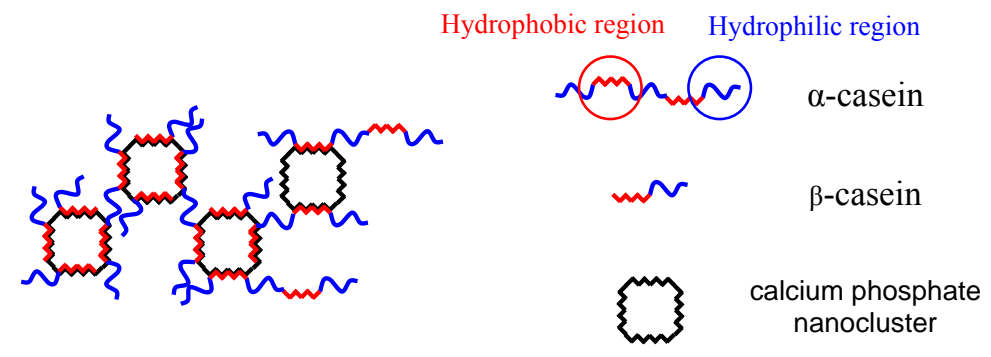

Figure 1.6: Illustration of network formation in the Holt model ${ }^{50}$. $\alpha$ - (both $\alpha_{\mathrm{s} 1}$ - and $\alpha_{\mathrm{s} 2}$-casein) and $\beta$-casein are shown as bi-functional and monofunctional, respectively. The calcium phosphate nanocluster is drawn as having four closure sites. The $\alpha$-casein act as bridges through binding to different nanoclusters and allows the chain to grow further through branch development. This is shown on the right hand side of the illustration. On the left hand side, the chain has closed.

Recently, Horne proposed the dual bonding model and claimed that the self-association of the caseins is also driven by electrostatic repulsive interactions in addition to hydrophobic interactions $^{10,56}$. Two types of linkage between protein molecules are postulated. First, two or more hydrophobic regions from different molecules form a bonded cluster. Further growth is inhibited by the protein charged residues. Second, he viewed the micellar calcium phosphate not just as cross-links but also as neutralising agents which, being positively charged $^{57}$, bind to negatively charged phosphoseryl clusters leading to the domination of the attractive interactions between the hydrophobic regions of the caseins. It is considered that up to four or more from different casein molecules can be accommodated at each calcium phosphate nanocluster ${ }^{58}$. The growth of the micelle is thus envisaged as depicted in Fig. 1.7.

\subsubsection{Aggregation of Commercial Casein}

Caseinate derived from native casein are mixtures of $\alpha-, \beta$-, and $\kappa$-casein in the ratios of $5: 4: 1^{59}$. Commercial casein protein molecules in aqueous dispersions exist as a polydisperse mixture of monomers, complexes and aggregates ${ }^{60}$ exhibiting compact quaternary structure through formation of subparticles via hydrophobic interactions (and hydrogen-bonding). It 
was shown, based on multiangle laser light scattering data that $\mathrm{Na}$-caseinate in solutions have radii of gyration $\left(\mathrm{R}_{\mathrm{g}}\right)$ values ranging from $\sim 50$ to $120 \mathrm{~nm}^{60}$. In charged subparticles, strong local attractive forces are expected to counteract the overall repulsive force that exists between molecules, suggesting subparticle aggregation. It has been found that the association of subparticles leads to the formation of complex supramolecular structure ${ }^{61}$.

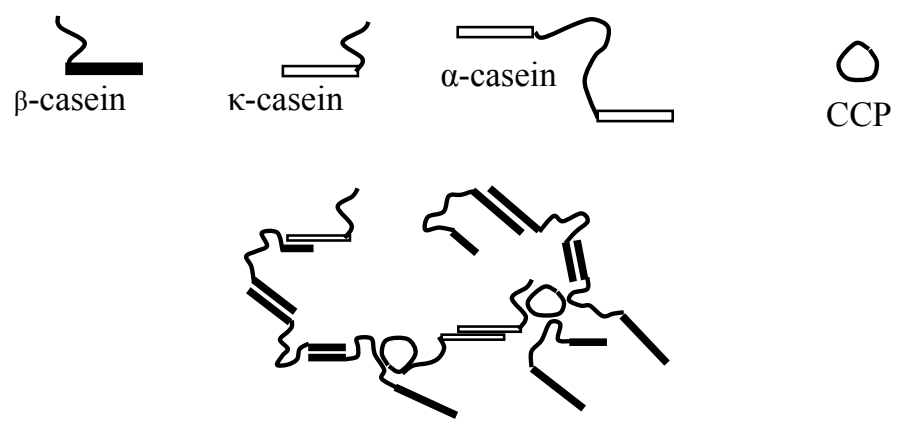

Figure 1.7: Dual bonding model for the structure of the casein micelle taken from 10, with $\alpha$-, $\beta$ - and $\kappa$-casein depicted as indicated. Bonding occurs between the hydrophobic regions, shown as rectangular bars, and by linkage of hydrophilic regions containing phophoseryl clusters to colloidal calcium phosphate clusters (CCP).

Farrer and Lips presented a detailed study of the osmotic pressure of semi-dilute and highly concentrated Na-caseinate solutions ${ }^{59}$. For the semi-dilute regime, the data at higher temperature $\left(>30^{\circ} \mathrm{C}\right)$ is considered in relation to the adhesive hard sphere model. The polycondensation theory provides better fits for data obtained at $30^{\circ} \mathrm{C}$. Both models suggest small submicellar building blocks of diameter c.a. $11 \mathrm{~nm}$ and aggregation number of 4 to 5 caseinate molecules with implicit voluminosity comparable to that of native casein submicelles. In the highly concentrated regime, a rigid lattice model is proposed which suggests "soft sphere" potentials for the submicelles.

The aggregation of casein increases with a decrease in ionic strength and/or an increase of $\mathrm{pH}$, the latter being quantitatively the most significant ${ }^{61}$. Higher $\mathrm{pH}$ increases the net negative charge and particle repulsion. However, the increase in negative charges induces particle expansion. This is believed to expose positively charged residues, responsible for subparticle association. The schematic representation of this argument is depicted in Fig. 1.8.

Ionisable residues passing through a maximum on increasing $\mathrm{pH}$ above the isoelectric point and van der Waals forces, i.e., permanent dipole-permanent dipole forces, permanent dipole-induced dipole forces and induced dipole-induced dipole forces contribute to the attraction energy between subparticles ${ }^{62}$. In dispersions with different ionic strength and $\mathrm{pH}$, solvent properties (solvent activity) complicate the variation of these forces. 


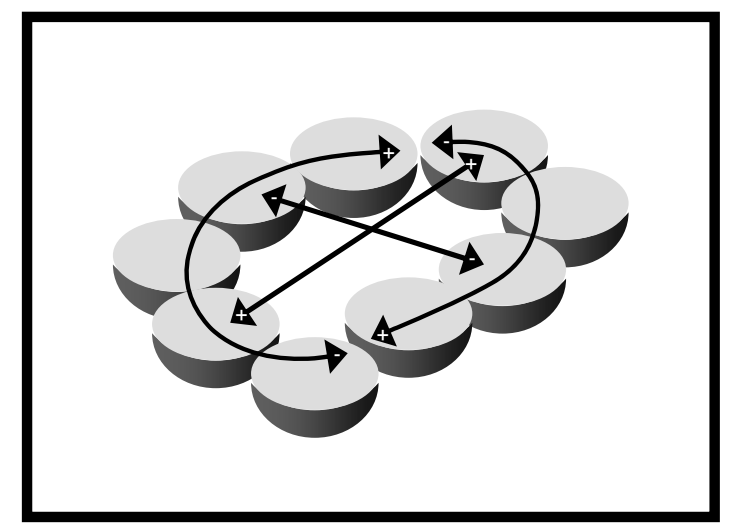

Figure 1.8: Schematic cross section of aggregated subparticles showing attractive and repulsive electrostatic forces stabilising the structure ${ }^{61}$.

\section{$1.2 \quad$ Emulsions}

As casein proteins are an important ingredient used in the formulation of food emulsions, what follows is a discussion on the concept of emulsions.

An emulsion consists of droplets of oil that are stabilised in an aqueous continuum by the presence of a series of emulsifying agents, which reside at the oil/water interface ${ }^{63}$. These emulsifiers can take the form of simple surfactants (charged or non-charged), polymers ${ }^{62,64}$, proteins $^{65}$ or finely divided solid ${ }^{66}$. Micro- and macroemulsions are among the major types of emulsions used in industry, including in food, paints, cosmetics and pharmaceuticals ${ }^{67-71}$. A comparison between the two different emulsion classes is given in Table 1.1.

When macroemulsions (here after referred to as just emulsions) are formed, the change in Gibbs energy, $\Delta G_{f}$ is expressed as

$$
\Delta G_{f}=\gamma \Delta A-T \Delta S
$$

where $\gamma$ is the interfacial tension between the oil and the water, $\Delta A$ is the change in interfacial area upon emulsification, $T$ is the temperature and $\Delta S$ is the change in entropy of the system. In emulsions the interfacial area between the oil and water domains is very large and the addition of an emulsifier is used to sufficiently reduce the interfacial tension thereby ensuring a limited emulsion lifetime ${ }^{72}$. The $\gamma \Delta A$ term is therefore the dominant favourable component and a positive energy is achieved. As such, emulsions are thermodynamically unstable but kinetically ${ }^{73,74}$ stable, requiring energy input to induce their formation, i.e., the process is not spontaneous ${ }^{75-77}$. 
Table 1.1: Some characteristic of microemulsions and (macro-) emulsions ${ }^{78}$

\begin{tabular}{lll}
\hline & Microemulsions & Emulsions \\
\hline appearance & clear & turbid-milky \\
diameter (nm) & 8 to 15 & 100 to 10000 \\
thermodynamic stability & stable & unstable \\
surface area & large & small \\
curvature & high & low \\
dispersity & monodisperse & polydisperse \\
solubilisation capability & high & low \\
\hline
\end{tabular}

Emulsions can be characterised as Winsor I, II, III and IV. In Fig. 1.9 are shown the four types of Winsor emulsions. Other types of emulsions include multiemulsions, e.g., water-oil-water $^{79,80}$ or oil-water-oil ${ }^{81}$ emulsions, biliquid foams or the so-called gel emulsions $^{82-85}$ which are formed at high volume fractions $>0.74$ in monodispersed undistorted systems, and nanoemulsions ${ }^{86,87}$ that are transparent with low viscosity but are still metastable. In this work, we focus on Winsor IV emulsions which contain no excess oil or water and are macroscopically monophasic upon preparation.

(a)

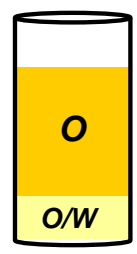

(b)

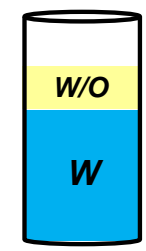

(c)

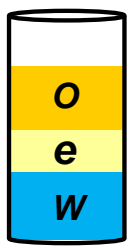

(d)

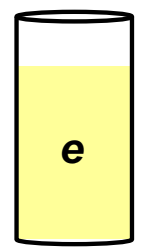

Figure 1.9: (a) Winsor I, (b) II, (c) III and (d) IV emulsions. Winsor IV emulsions may be oil-in-water (O/W) or water-in-oil $(W / O)$ emulsions. Note that $O, W$ and $e$ represent oil, water and emulsion, respectively.

Depending on the types of emulsions required, the ease of use, availability and cost, emulsions can be formed via shaking, stirring, milling and/or homogenisation. All methods used in emulsion formulation create either bounded or unbounded flow. Bounded flow is induced if the droplets formed are much smaller than the compartment of the apparatus, e.g., pipe flow, static mixer and stirrer. Unbounded flow occurs when the emulsion is formed in an apparatus with a dimension similar to the droplet size of the droplets formed, e.g., homogeniser. The latter was used exclusively throughout this research for the formation of emulsions based on a mixture of oil and Na-caseinate; the dispersion is passed through a very 
narrow slit and the homogeniser converts potential energy to kinetic energy.

\subsection{Stability of Emulsion}

Emulsions will, given sufficient time, phase separate. Hence, emulsion lifetime is one of the factors of most concern ${ }^{64,71,88}$. The stability of emulsions generally depends on the Gibbs elasticity $^{89-91}$, Gaussian curvature ${ }^{92,93}$, bending energy, Laplace pressure differential ${ }^{94}$ and intermolecular interactions between the emulsifier and the oil. Each of these varies as a function of concentration and may be codependent. If these controlling factors were not mutually satisfied, emulsions could not be stabilised. We will consider each of these in turn.

\subsubsection{Gibbs Elasticity}

Elasticity is normally referred to as the ratio of stress to strain. It is a measure of the ability of the system to "self-heal" against external disturbance, i.e., to return to an initial state or form following deformation such as stretching. In the context of an emulsion, the elasticity, i.e., the strength of a thin film formed at an oil/water interface is often denoted as the Gibbs elasticity $(E)$, the ratio of the change in surface tension to the change in film surface area:

$$
E=2 A \frac{d \gamma}{d c} \frac{d c}{d A}
$$

where $A$ is the film surface area, $\gamma$ is the interfacial tension and $c$ is the bulk surfactant concentration.

Christenson and Yaminsky ${ }^{95}$ made a simplify assumption and derived a model to calculate Gibbs elasticities from surface tension gradients $\left(\frac{d \gamma}{d c}\right)$. Using the Gibbs adsorption isotherm, Wang and Yoon ${ }^{96}$ developed a generalised model that can be applied to films at any surfactant concentration. They expressed $E$ as follows:

$$
E=\frac{4 R T \Gamma^{2}}{c\left(H+2 \frac{d \Gamma}{d c}\right)}
$$

where $R$ is the gas constant, $T$ is the temperature, $\Gamma$ is the surface excess of a surfactant at 
an air/water interface and $H$ is the film thickness. Equation 1.4 can be transformed into a more useful form ${ }^{96}$ :

$$
E=\frac{4 R T \Gamma_{m}^{2} K_{L}^{2} c}{H\left(1+K_{L} c\right)^{2}+2 \Gamma_{m} K_{L}}
$$

where $\Gamma_{m}$ is the maximum adsorption density and $K_{L}$ is the equilibrium adsorption constant. By fitting the surface tension data to the Langmuir isotherm, $\Gamma=\frac{\Gamma_{m} K_{L} c}{1+K_{L} c}$ and determining $\Gamma_{m}$ and $K_{L}$, the Gibbs elasticity can be obtained from equation 1.5.

Considering equation 1.3, a thin film formed at an oil/water interface, which locally has a lower emulsifier concentration and hence a higher interfacial tension posses a high elasticity. In other words, Gibbs elasticity reflects the preferential adsorption of emulsifier. For stabilisation of emulsions against flocculation and coalescence, strong adsorption (or "anchoring") of the emulsifier molecule to the surface of the droplet is required. At low emulsifier concentration, Gibbs elasticity increases due to the increase in adsorbed matter. The interfacial film automatically strengthens itself wherever a break may occur. The higher molecular weight of the emulsifier would mean a lower diffusion rate of the emulsifier, and therefore the dynamic interfacial tension would be higher than the equilibrium value ${ }^{97}$.

\subsubsection{Gaussian Curvature}

In order to understand Gaussian curvature, $K$, we must first understand the concept of curvature. At a given (and any) point on a curve or surface, the line best approaching the curve that passes through the point is the tangent line. Similarly, the best approximating circle that passes through that point is also tangent to the curve. The reciprocal of the radius of this circle is the curvature of the curve at that point. The best approximating circle may lie either to the left or to the right of the curve. Giving the curvature a positive or a negative sign if the circle lies to the left and to the right of the curve, respectively, this is known as the signed curvature. In the context of an oil/water interface, a positive or a negative signed curvature is a curvature that curves toward the oil and the water domains, respectively.

At a given point on the surface and for a direction lying in the tangent plane of the surface at that point, the normal section curvature can be computed by intersecting the surface with the plane spanned by the point, the normal to the surface at that point, and the 
direction (lying in the tangent plane of the surface) are then determined. The normal section curvature is the signed curvature of the curve at the point of interest. Considering all directions in the tangent plane to the surface at that point, one can compute the normal section curvature in all these directions. A maximum and a minimum value will be obtained. Gaussian curvature ${ }^{98,99}$ is then the product of these maximum and minimum values.

The Gaussian curvature, $K=K_{1} K_{2}$ where $K_{1}$ and $K_{2}$ are the principal curvatures, $K_{1}=\frac{1}{R_{1}}, K_{2}=\frac{1}{R_{2}}$ with $R_{1}$ and $R_{2}$ are the principal radii of curvatures, named after Carl Friedrich Gauss, has the dimension of $\frac{1}{\text { length }^{2}}$. It is positive (from the product of two maximum or two minimum values) for spheres, negative (from the product of one maximum and one minimum values) for one-sheet hyperboloids and zero for planes and cylinders. It determines whether a surface is locally convex, i.e., either a peak or a valley (when it is positive) or locally saddle (when it is negative). An illustration on the concept of Gaussian curvature is shown in Fig. 1.10.

(a)

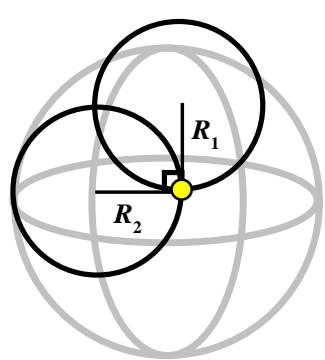

(b)

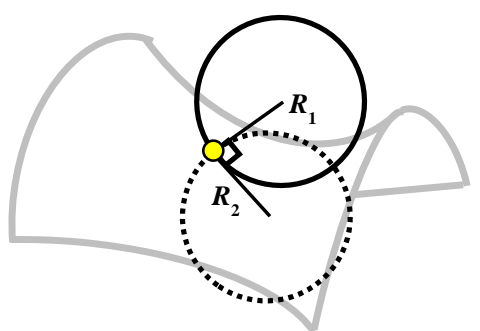

Figure 1.10: Illustration of the concept of Gaussian curvature. The reference point on a surface (sphere and saddle) is denoted as a yellow dot. Consider: (a) two circles (solid black) that are located above the sphere, perpendicular to each other and pass through the reference point, the product of the reciprocal radii of the two circles yields a positive Gaussian curvature and (b) two circles that are located above (solid black) and below (dotted black) the saddle, perpendicular to each other and pass through the reference point, the product of the reciprocal radii of the two circles yields a negative Gaussian curvature.

In emulsions, a positive and negative signed curvature corresponds to curvatures that curve toward the oil and the water domain, respectively. A positive and/or a negative signed curvature could exist simultaneously in an emulsion. Positive, negative and near zero Gaussian curvatures are realisable and correspond to oil-in-water emulsions, water-in-oil emulsions and bicontinuous emulsions and lamellar phases. Gaussian curvature also 
determines the stability of emulsions. In particular, when coalescence occurs, i.e., the fusion of two droplets, one of which has a positive signed curvature (curvature curves towards the oil domain) and the other has a negative signed curvature (curvature curves towards the water domain), the Gaussian curvature is negative. By determining the destabilisation rate, one can indirectly identify the types of Gaussian curvature formed in the system.

\subsubsection{Bending Energy}

Interfacial curvature and bending energy are two inter-related variables. Variation of the curvature causes a change in bending energy ${ }^{100,101}$. The bending energy of a spherical interface, for example, can be expressed as:

$$
W_{c}=2 \pi a^{2} B_{0} H
$$

where $a$ is the film radius, $B_{0}=-4 k_{c} H_{0}$, is the interfacial bending moment of a flat interface with $k_{c}$ as the bending elasticity constant, $H_{0}=-\frac{1}{R_{0}}$, as the spontaneous curvature of the interface and $R_{0}$ is the principal radius of curvature. $H=-\frac{1}{r}$, is the interfacial curvature with $r$ as the droplet radius and $\left(\frac{a}{r}\right) \ll 1$.

It has been suggested that the films of two droplets in near contact flatten on approaching each other, the thickness of the films then decrease before they rupture and the two droplets coalesce ${ }^{102}$. Positive $B_{0}$ opposes the flattening of the two droplet surfaces in the zone of collision, but negative $B_{0}$ favours the flattening. For oil-in-water emulsions, the droplets serve as adhesives for the other emulsion droplets. The collision of two droplets leads to the spontaneous formation of a doublet of two droplets due to the effect of bending energy and flocculation occurs. In contrast, for water-in-oil emulsions the local dilatation of the interface in the zone of contact favours the appearance of bridging particles causing coalescence of the two droplets. 


\subsubsection{Laplace Pressure}

Laplace's law describes the relationship between the transmural pressure difference and the tension, radius, and thickness of closed films:

$$
T=\frac{(P \cdot R)}{M}
$$

where $T$ is the tension in the walls, $P$ is the pressure difference across the wall, $R$ is the radius of the films and $M$ is the thickness of the wall. Obviously, the higher the pressure difference and the larger the radius, the more tension there will be. On the other hand, the thicker the wall the less tension there is.

Consider the Laplace pressure differential $(\Delta p)$ between the inside and outside of a spherical droplet. It has the following form:

$$
\Delta p=\frac{2 \gamma}{R}
$$

where $\gamma$ is the interfacial tension and $R$ is the radius of the spherical droplet. The most stable system is when $\Delta p=0$. In practice, small droplets coated with sufficient emulsifier forming a reasonably thick "wall" are preferred to minimise the effect of Laplace pressure, resulting in a stable emulsion. Laplace pressure controls droplet deformation. During destabilisation processes, for example in an oil-in-water emulsion, small droplets lose oil causing compression of the adsorbed particle layer with a concomitant lowering of interfacial tension. Large droplets swell with additional oil and adsorption of emulsifier from the bulk to cover the new interfaces, the interfacial tension will increase. The whole process is arrested when the Laplace pressure of different size droplets becomes equal, thereby reducing the driving force for destabilisation to zero.

\subsection{Casein-stabilised Emulsions}

Protein-stabilised food emulsions constitute the most important class of food colloids ${ }^{103,104}$. In particular, the caseinates, composed of $\alpha_{\mathrm{s} 1^{-}}, \alpha_{\mathrm{s} 2^{-}}, \beta-$, and $\kappa$-casein are the most widely used milk proteins in emulsion formation. The properties of the caseins have been examined and reported in the literature. For example, in water dispersions, large casein complexes were found to form comprised of 16 and even 50 casein monomers ${ }^{13}$. Centrifugal sedimentation, 
size-exclusion chromatography, electron microscopy and light scattering techniques have been used to study the aggregation behaviour of casein monomers ${ }^{17,105-114}$.

Tai et al. ${ }^{106}$ used and extended the shell model to predict the moving boundary sedimentation behaviour of micellar $\beta$-casein. The observed sedimentation patterns were satisfactorily described with the shell model, based on a continuous distribution of intermediates between monomers and the largest possible spherical micelles.

Using light scattering, Schmidt et al. ${ }^{107}$ investigated the association behaviour of $\alpha_{\mathrm{s} 1}{ }^{-}$ and $\beta$-casein at very low protein concentrations. The association behaviour of both $\alpha_{\mathrm{s} 1}$ - and $\beta$-casein appeared to be quite different. $\alpha_{\mathrm{s} 1}$-casein undergoes a series of consecutive association steps whereas $\beta$-casein forms polymers of a very high degree of polymerisation. From thermodynamic considerations it was inferred that hydrophobic bonding is the main driving force in the association of the caseins. Gel filtration chromatography ${ }^{105}$ at $36^{\circ} \mathrm{C}$ showed that $\beta$-casein is large with a molecular weight of $1.34 \times 10^{6}$. Small-angle neutron scattering profiles further show that $\beta$-casein micelles are spherical ${ }^{17,114}$ with an almost constant radius of $135 \AA$. They consist of a relatively large and dense core surrounded by a shell of much lower density. Though its density increases with the aggregation number it is never compact. Evans et al. ${ }^{109}$ and Berry et al. ${ }^{105}$ on the other hand studied the effect of modifying the hydrophobic/hydrophilic balance on the micellisation of $\beta$-casein. This modification drastically reduces the ability of the $\beta$-casein molecule to form micelles.

While it is known that casein proteins self-assemble to form micelles in aqueous calcium solution, their behaviour in native buffer solution has not been widely investigated and certainly not beyond the dilute regime. The formation of higher orders of casein structures in aqueous solution were generally recognised to have considerable influence over either/both the formulation characteristics for emulsion synthesis or the final characteristics of the emulsion ${ }^{115,116}$. As such, the first objective of this programme was to determine the relationship between the extent of aggregation and gelation of casein aggregates and the state of water molecules resulting from water-protein as well as protein-protein interactions in aqueous dispersions which then determine the microstructure and flow behaviours of the resulting soft matter.

Owing to the importance of caseins in industrial applications ${ }^{4,117-119}$, studies have been carried out in model emulsions ${ }^{20,21,25,26,120-131}$ to understand the adsorption behaviour of individual caseins and/or casein aggregates at the surface of newly formed droplets and its correlation with the emulsion stability ${ }^{2}, 19,125,132,133$. Physical properties of oil-in-water 
emulsions stabilised by caseins are determined largely by the nature of the adsorbed layer at the surface of the dispersed droplets. Analysis of the aqueous phase after centrifugation of fresh emulsions made with a mixture of $\alpha_{\mathrm{s} 1}$ - and $\beta$-casein shows that $\beta$-casein predominates at the interface ${ }^{26}$. Addition of calcium chloride to Na-caseinate solutions above $0.08 \% \mathrm{w} / \mathrm{w}$ resulted in formation of large casein particles/aggregates which adsorbed to on the droplet surface causing higher surface protein concentrations.

Dickinson et al. ${ }^{133}$ found that the creaming kinetics of oil-in-water emulsions (30 or 45 vol\% oil) has a complex dependence on Na-caseinate content. At low protein content, the emulsion is destabilised by bridging flocculation due to low oil droplet surface coverage. At higher protein content, the emulsion has good stability. Further increasing protein content reduces the emulsion stability. This is attributed to depletion flocculation caused by unadsorbed caseinate. Once the caseinate concentration reaches a high value, it produces a very strong emulsion droplet network and hence the emulsion is much more stable. At caseinate concentration below $2 \mathrm{wt} \%, \beta$-casein was found to adsorb at the surface of oil droplets in preference to other caseins ${ }^{25}$. Increasing the oil concentration from 10 to $30 \mathrm{wt} \%$ decreased the protein load at droplet surfaces from 3.7 to $1.4 \mathrm{mg} \mathrm{m}^{-2}$, but further increases in oil concentration had much less effect. A decrease in protein load was observed as the homogenisation pressure increased from 34 to 136 bar.

Moreover, useful insight into properties of adsorbed layers at oil/water interface has been obtained by examining the flow response of emulsions ${ }^{132,134}$. It was demonstrated that the transition of the state of the adsorbed layer due to, for instance, the formation of flocs and emulsion networks induced by the presence of non-adsorbing proteins ${ }^{135,136}$ is accompanied by a change in the flow pattern of emulsions ${ }^{137,138}$. Rheology of dilute caseinate emulsions reported in the literature is often limited to a narrow range of shear rates, based on this data at low shear rates, the system is shear-thinning but is Newtonian at higher shear rates ${ }^{137-141}$. Note that shear-thinning behaviour is observed when the domains of aggregated structure break up resulting in the release of temporarily trapped continuous phase of the emulsion. This is indicated by a reduction in the viscosity on increasing shear rates.

Formation of a viscoelastic layer due to protein adsorption ${ }^{142-145}$ and entanglements ${ }^{146-}$ 148 at the interface in emulsions has been previously reported. In particular, smalldeformation rheology showed that salad dressing type emulsions ${ }^{149}{ }^{150}$, commercial mayonnaise ${ }^{150-154}$ and pea-protein stabilised concentrated oil-in-water emulsions ${ }^{152}$ exhibit solid-like behaviour. Non-linear rheology studies of concentrated emulsions showed that 
yield stress may be present in the system ${ }^{155,156}$. Moreover, the flow behaviour of these systems is rather complex because recently it was observed that concentrated emulsions undergo a transition from solid-like to fluid-like under shear ${ }^{157-164}$.

Compared to dilute emulsions, rheology of concentrated emulsions containing $>50 \mathrm{wt} \%$ oil has received less attention. In addition, rheology of concentrated emulsions is also restricted to a narrow range of shear rates. While concentrated emulsions are known to have similar properties with gels, foams, pastes and suspensions, the detailed emulsion characteristics $^{165,166}$ which are defined by the emulsifier ${ }^{167}$, dispersed phase and continuous phase $^{80,82,87}$ has yet to be fully elucidated. Therefore, the second objective of this programme is an investigation of the effect of casein concentration on the stability, microstructure and flow properties in concentrated emulsions comprised of a long-chain oil (tetradecane) or commercially-used oils (soybean oil and palm oil) on the formation and stability of the casein-stabilised emulsions. Comparison of the surface and emulsifying characteristics of Na-caseinate in the presence of different types of oils is worthwhile for two reasons. Firstly, since straight chain oils cannot be used in dairy-based food products, it is interesting to compare their behaviour with that of food grade oil. Secondly, there is a practical issue of ingredient development. Continuing oil refinements in separation technology offers for future commercial use new oil fractions, e.g., palm oil, which may be substantially enriched in dairy food.

By evaluating the as-prepared emulsions and relating the combined effects to the physicochemical properties of the system, it is envisaged that a better understanding of the nature of the microstructure in directing emulsion characteristics can be obtained. To achieve this, the $\mathrm{pH}$, ionic strength, temperature and concentration must be at a level such that emulsion microstructure and stability is thermodynamically controlled. Working in this area should emphasise the role of the different modes of complementarity on the interfacial domain and thereby the stability of the concentrated emulsions. Four main techniques will be used to characterise both the casein in buffer system and the concentrated emulsions; details of each are given in the following chapter. 


\subsection{References}

1. G. Doxastakis, in Food emulsifiers, edited by G. Charalambous and G. Doxastakis (Elsevier, Amsterdam, 1989), pp. 9-62.

2. E. Dickinson, An introduction to food colloids. (Oxford University Press, Oxford, 1992).

3. J. E. Kinsella, CRC Crit. Rev. Food Sci. Nutr. 21, 197-262 (1984).

4. C. V. Morr, in Developments in dairy chemistry-1, edited by P. F. Fox (Applied Science, London, 1982), pp. 375-399.

5. O. Tossavainen, P. Pyykkönen, P. Vastamäki and H. Huotari, Int. Dairy J. 6, 171-184 (1996).

6. J. E. Kinsella, CRC Crit. Rev. Food Sci. Nutr. 21, 197-262 (1988).

7. L. L. Muller, in Developments in dairy chemistry-1, edited by P. F. Fox (Applied Science, London, 1982), pp. 315-337.

8. D. G. Dalgleish, Int. Dairy J. 14, 1025-1031 (2004).

9. D. S. Horne, Curr. Opin. Colloid Interface Sci. 11, 148-153 (2006).

10. D. S. Horne, Int. Dairy J. 8, 171-177 (1998).

11. M. Panouillé, D. Durand, T. Nicolai, E. Larquet and N. Boisset, J. Colloid Interface Sci. 287, 85-93 (2005).

12. T. H. M. Snoeren, B. V. Markwijk and R. V. Montfort, Biochim. Biophys. Acta 622, 268-276 (1980).

13. D. G. Schmidt, in Developments in dairy chemistry-1, edited by P. F. Fox (Applied Science Publishers, England, 1982), pp. 61-86.

14. T. A. J. Payens, J. Brinkhuis and B. W. V. Markwijk, Biochim. Biophys. Acta 175, 434-437 (1969).

15. A. Thurn, W. Burchard and R. Niki, Colloid Polym. Sci. 265, 897-902 (1987).

16. H. E. Swaisgood, in Developments in dairy chemistry, edited by P. F. Fox (Elsevier, London, 1982), Vol. 1, pp. 1-59.

17. A. Thurn, W. Burchard and R. Niki, Colloid Polym. Sci. 265, 653-666 (1987).

18. H. E. Swaisgood, in Advanced dairy chemistry, edited by P. F. Fox and P. L. H. McSweeney (Kluwer Academic/Plenum Publishers, New York, 2003), pp. 139-201.

19. E. Dickinson, J. Chem. Soc., Faraday Trans. 88, 2973-2982 (1992). 
20. E. Dickinson, J. Dairy Res. 56, 471-477 (1989).

21. E. W. Robson and D. G. Dalgleish, J. Food Sci. 52, 1694-1698 (1987).

22. D. S. Horne, Curr. Opin. Colloid Interface Sci. 7, 456-461 (2002).

23. E. Dickinson and M. Lal, Adv. Mol. Related Interaction Pro. 17, 1-87 (1980).

24. G. J. Fleer, M. A. C. Stuart, J. M. H. M. Scheutjens, E. Cosgrove and B. Vincent, Polymers at interface. (Chapman \& Hall, London, 1993).

25. M. Srinivasan, H. Singh and P. A. Munro, J. Agric. Food Chem. 44, 3807-3811 (1996).

26. E. Dickinson, S. E. Rolfe and D. G. Dalgleish, Food Hydrocolloids 2, 397-405 (1988).

27. J.-L. Courthaudon, E. Dickinson and D. G. Dalgleish, J. Colloid Interface Sci. 145, 390-395 (1991).

28. E. Dickinson, Int. Dairy J. 9, 305-312 (1999).

29. V. B. Fainerman, R. Miller and R. Wüstneck, J. Colloid Interface Sci. 183, 26-34 (1996).

30. E. Dickinson, S. E. Rolfe and D. G. Dalgleish, Int. J. Biol. Macromol. 12, 189-194 (1990).

31. D. C. Clark, F. Husband, P. J. Wilde, M. Cornec, R. Miller, J. Krägel and R. Wüstneck, J. Chem. Soc., Faraday Trans. 91, 1991-1996 (1995).

32. E. Dickinson, B. S. Murray and G. Stainsby, J. Chem. Soc., Faraday Trans. 84, 871883 (1988).

33. D. C. Clark, A. R. Mackie, P. J. Wilde and D. R. Wilson, in Food proteins: structure and functionality, edited by K. D. Schwenke and R. Mothes (VCH, Weinheim, New York, 1993), pp. 263-269.

34. D. C. Clark, P. J. Wilde, D. J. M. Bergink-Martens, A. J. J. Kokelaar and A. Prins, in Food colloid and polymers: stability and mechanical properties, edited by E. Dickinson and P. Walstra (Royal Society of Chemistry, Cambridge, 1993), pp. 354364.

35. E. Dickinson, D. S. Horne, J. S. Phipps and R. M. Richardson, Langmuir 9, 242-248 (1993).

36. D. G. Dalgleish, Colloids Surfs. B 1, 1-8 (1993).

37. D. G. Dalgleish, Colloids Surfs. 46, 141-155 (1990).

38. T. Nylander and M. Wahlgren, J. Colloid Interface Sci. 162, 151-162 (1994).

39. M. Britten and H. J. Giroux, J. Agric. Food Chem. 41, 1187-1191 (1993). 
40. J. Leaver, D. V. Brooksbank and D. S. Horne, J. Colloid Interface Sci. 162, 463-469 (1994).

41. Y. Fang and D. G. Dalgleish, J. Colloid Interface Sci. 156, 329-334 (1993).

42. D. G. Dalgleish, Food Res. Int. 29, 541-547 (1996).

43. M. Shimizu, A. Ametani, S. Kaminogawa and K. Yamauchi, 869, 259-264 (1986).

44. J. Leaver and D. G. Dalgleish, Biochim. Biophys. Acta 1041, 217-222 (1990).

45. J. E. O'Connell, V. Y. Grinberg and C. G. de Kruif, J. Colloid Interface Sci. 258, 3339 (2003).

46. D. G. Schmidt, in Developments in dairy chemistry-1, edited by P. F. Fox (Applied Science Publishers, London, 1982), pp. 61-82.

47. P. Walstra, J. Dairy Sci. 73, 1965-1979 (1990).

48. C. Holt, P. A. Timmins, N. Errington and J. Leaver, Eur. J. Biochem. 252, 73-78 (1998).

49. C. G. deKruif, Int. Dairy J. 9, 183-188 (1999).

50. P. F. Fox, in Advanced dairy chemistry, edited by P. F. Fox and P. L. H. McSweeney (Kluwer Academic/Plenum Publishers, New York, 2003), pp. 1-48.

51. C. W. Slattery and R. Evard, Biochim. Biophys. Acta 317, 529-538 (1973).

52. C. W. Slattery, Biophys. Chem. 6, 59-64 (1977).

53. D. G. Schmidt, Neth. Milk Dairy J. 34, 42-64 (1980).

54. C. G. deKruif and C. Holt, in Advanced dairy chemistry, edited by P. F. Fox and P. L. H. McSweeney (Kluwer Academic/Plenum Publishers, New York, 2003), pp. 213276.

55. C. Holt, Eur. Biophys. J. 33, 421-434 (2004).

56. D. S. Horne, in Milk proteins: from expression to food, edited by A. Thompson, M. Boland and H. Singh (Academic Press, Elsevier, 2008).

57. D. G. Schmidt, in Developments in dairy chemistry 1-Proteins, edited by P. F. Fox (Applied Science Publishers, London, 1982), pp. 61-85.

58. C. Holt, in Advances in protein chemistry, edited by C. B. Anfinsen, J. D. Edsall, F. R. Richards and D. S. Eisenberg (Academic Press, San Diego, 1992), Vol. 43, pp. 63 151.

59. D. Farrer and A. Lips, Int. Dairy J. 9, 281-286 (1999).

60. J. A. Lucey, M. Srinivasan, H. Singh and P. A. Munro, J. Agric. Food Chem. 48, 1610-1616 (2000). 
61. M. Boulet, M. Britten and F. Lamarche, Food Hydrocolloids 14, 135-144 (2000).

62. J. Lindman and H. Kronberg, Surfactants and polymers in aqueous solution. (John Wiley \& Sons, New York, 1999).

63. V. Schmitt, C. Cattelet and F. Leal-Calderon, Langmuir 20, 46-52 (2004).

64. S. S. Davis, J. Colloid Interface Sci. 35, 665-674 (1971).

65. T. D. Dimitrova and F. Leal-Calderon, Langmuir 15, 8813-8821 (1999).

66. D. E. Tambe and M. M. Sharma, J. Colloid Interface Sci. 162, 1-10 (1994).

67. G. M. Eccleston, J. Colloid Interface Sci. 57, 66-74 (1976).

68. N. Diftis and V. Kiosseoglou, Food Hydrocolloids 18, 639-646 (2004).

69. J. John, M. Bhattacharya and P. C. Raynor, Colloids Surfs. A 237, 141-150 (2004).

70. E. Tsaliki, S. Pegiadou and G. Doxastakis, Food Hydrocolloids 18, 631-637 (2004).

71. E. Dickinson, J. Dairy Sci. 80, 2607-2619 (1997).

72. M. P. Aronson, Langmuir 5, 494-501 (1989).

73. R. Aveyard, B. P. Binks, J. Esquena, P. D. I. Fletcher, P. Bault and P. Villa, Langmuir 18, 3487-3494 (2002).

74. R. Aveyard, B. P. Binks, J. Esquena, P. D. I. Fletcher, R. Buscall and S. Davies, Langmuir 15, 970-980 (1999).

75. S. S. Dukhin, G. Kretzschmar and R. Miller, in Studies in interface science, edited by D. Möbius and R. Miller (Elsevier, Amsterdam, 1995), Vol. 1.

76. J. Benjamins and E. H. Lucassen-Reynders, in Studies in interface science, edited by D. Möbius and R. Miller (Elsevier, Amsterdam, 1998), Vol. 7, pp. 341-384.

77. M.-J. Rang and C. A. Miller, J. Colloid Interface Sci. 209, 179-192 (1999).

78. E. Dickinson, Food colloids. (The Royal Society of Chemistry, UK, 2005).

79. I. Lönnqvist, B. Hakansson, B. Balinov and O. Söderman, J. Colloid Interface Sci. 192, 66-73 (1997).

80. F. Cournarie, V. Rosilio, M. Cheron, C. Vauthier, B. Lacour, J.-L. Grossiord and M. Seiller, Colloid Polym. Sci. 282, 562-568 (2004).

81. R. Pal, Langmuir 12, 2220-2225 (1996).

82. C. Solans, J. Esquena and N. Azemar, Curr. Opin. Colloid Interface Sci. 8, 156-163 (2003).

83. O. Sonneville-Aubrun, V. Bergeron, T. Gulik-Krzywicki, B. Jönsson, H. Wennerström, P. Lindner and B. Cabane, Langmuir 16, 1566-1579 (2000). 
84. R. Mezzenga, J. Ruokolainen, G. H. Fredrickson and E. J. Kramer, Macromolecules 36, 4466-4471 (2003).

85. M. S. Spector, J. A. Zasadzinski and M. B. Sankaram, Langmuir 12, 4704-4708 (1996).

86. D. Morales, J. M. Gutierrez, M. J. Garcia-Celma and Y. C. Solans, Langmuir 19, 7196-7200 (2003).

87. O. Sonneville-Aubrun, J.-T. Simonnet and F. L'Alloret, Adv. Colloid Interface Sci. 108-109, 145-149 (2004).

88. T. D. Dimitrova, F. Leal-Calderon, T. D. Gurkov and B. Campbell, Langmuir 17, 8069-8077 (2001).

89. E. H. Lucassen-Reynders, A. Cagna and J. Lucassen, Colloids Surfs. A 186, 63-72 (2001).

90. A. I. Rusanov, V. V. Krotov and A. G. Nekrasov, Langmuir 20, 1511-1516 (2004).

91. A. Kabalnov and H. Wennerströem, Langmuir 12, 276-292 (1996).

92. A. Cacciuto, S. Auer and D. Frenkel, J. Chem. Phys. 119, 7467-7470 (2003).

93. S. Lee, D. H. Kim and D. Needham, Langmuir 17, 5537-5543 (2001).

94. D. Fennell Evans and H. Wennerström, The colloidal domain: where physics, chemistry, biology, and technology meet. (Wiley VCH, New York, 1994).

95. H. K. Christenson and V. V. Yaminsky, J. Phys. Chem. 99, 10420 (1995).

96. L. Wang and R.-H. Yoon, Colloids Surfs. A 282-283, 84-91 (2006).

97. E. H. Lucassen-Reynders, in Anionic surfactants: physical chemistry of surfactant action, edited by E. H. Lucassen-Reynders (Marcel Dekker, New York, 1981).

98. W. Kühnel, (American Mathematical Society, United States of America, 2006).

99. A. Gray, in Modern differential geometry of curves and surfaces with mathematica (CRC Press, Boca Raton, FL, 1997), pp. 373-380 and 481-500.

100. D. N. Petsev, N. D. Denkov and P. A. Kralchevsky, J. Colloid Interface Sci. 176, 201 213 (1995).

101. W. Helfrich, Z. Naturforsch. C 28, 693-703 (1973).

102. K. D. Danov, N. D. Denkov, D. N. Petsev, I. B. Ivanov and R. Borwankar, Langmuir 9, 1731-1740 (1993).

103. E. Dickinson, J. Chem. Soc., Faraday Trans. 88, 2973-2983 (1992).

104. P. J. Halling, CRC Crit. Rev. Food Sci. Nutr. 13, 155-203 (1981).

105. G. P. Berry and L. K. Creamer, Biochem. 14, 3542-3545 (1975). 
106. M. S. Tai and G. Kegelas, Biophys. Chem. 20, 81-87 (1984).

107. D. G. Schmidt and T. A. J. Payens, J. Colloid Interface Sci. 39, 655-662 (1972).

108. A. L. Andrews, D. Atkinson, M. T. A. Evans, E. G. Finer, J. P. Green, M. C. Phillips and R. N. Robertson, Biopolym. 18, 1105-1121 (1979).

109. M. T. A. Evans, M. C. Phillips and M. N. Jones, Biopolym. 18, 1123-1140 (1979).

110. S. Arima, R. Niki and K. Takase, J. Dairy Res. 46, 281-282 (1979).

111. W. Buchheim and D. G. Schmidt, J. Dairy Res. 46, 277-280 (1979).

112. K. Kajiwara, R. Niki, H. Urakana, Y. Hirayi, N. Donkai and M. Nagura, Biochim. Biophys. Acta 955, 128-134 (1988).

113. E. Leclerc and P. Calmettes, Phys. B 234, 207-209 (1997).

114. E. Leclerc and P. Calmettes, Phys. B 241, 1141-1143 (1998).

115. S. Damodaran, in Food proteins, edited by J. E. Kinsella and W. G. Soucie (The American Oil Chemists' Society, Champaign, Il, 1988), pp. 21-51.

116. J. E. Kinsella, in Food protein deterioration, edited by J. P. Cherry (American Chemical Society, Washington, DC, 1982), pp. 301-325.

117. D. M. Mulvihill and P. F. Fox, in Developments in dairy chemistry-4, edited by P. F. Fox (Elsevier Applied Science, London, 1989), pp. 131-172.

118. L. G. Phillips, D. M. Whitehead and J. Kinsella, Structure-function properties of food proteins. (Academic Press, San Diego, 1994).

119. D. G. Dalgleish, in Food macromolecules and colloids, edited by E. Dickinson and D. Lorient (Royal Society of Chemistry, London, 1995), pp. 23-33.

120. E. Dickinson, A. Mauffret, S. Rolfe and C. M. Woskett, J. Soc. Dairy Technol. 42, 18-22 (1989).

121. P. Walstra, in Encyclopedia of emulsion technology, edited by P. Becher (Marcel Dekker, New York, 1983), Vol. 1, pp. 57-127.

122. P. Walstra and I. Smulders, in Food colloids: proteins, lipids and polysaccharides, edited by E. Dickinson and B. Bergenståhl (Royal Society of Chemistry, Cambridge, 1997), pp. 367-381.

123. D. M. Mulvihill and P. C. Murphy, Int. Dairy J. 1, 13-37 (1991).

124. E. Dickinson, J. Chem. Soc., Faraday Trans. 94, 1657-1669 (1998).

125. E. Dickinson, V. J. Pinfield, D. S. Horne and F. A. M. Leermakers, J. Chem. Soc., Faraday Trans. 93, 1785-1790 (1997).

126. M. Srinivasan, H. Singh and P. A. Munro, Int. Dairy J. 9, 337-341 (1999). 
127. S. Damodaran and A. Paraf, Food proteins and their applications. (Marcel Dekker, New York, 1977).

128. E. Tornberg, J. Sci. Food Agric. 29, 762-776 (1978).

129. J. A. Hunt and D. G. Dalgleish, Food Hydrocolloids 8, 175-187 (1994).

130. E. Dickinson, B. S. Murray and G. Stainsby, in Advances in food emulsions and foams, edited by E. Dickinson and G. Stainsby (Elsevier, Amsterdam, 1988), pp. 123162.

131. E. Tornberg, J. Sci. Food Agric. 46, 93-114 (1978).

132. E. Dickinson and G. Stainsby, Colloids in food. (Applied Science, London, 1982).

133. E. Dickinson, M. Golding and M. J. W. Povey, J. Colloid Interface Sci. 185, 515-529 (1997).

134. B. S. Murray and E. Dickinson, Food Sci. Technol. Int. (Japan) 2, 131-145 (1996).

135. E. Dickinson, M. I. Goller and D. J. Wedlock, J. Colloid Interface Sci. 172, 192-202 (1995).

136. R. Pal, Colloids Surfs. A 64, 207-215 (1992).

137. E. Dickinson and J. Chen, J. Agric. Food Chem. 16, 91-97 (1998).

138. E. Dickinson and M. Golding, J. Colloid Interface Sci. 197, 133-141 (1998).

139. G. B. Thurston and S. S. Davis, J. Colloid Interface Sci. 69, 199-208 (1979).

140. C. G. Quintero, C. Noik, C. Dalmazzone and J.-L. Grossiord, Rheol. Acta 47, 417-424 (2008).

141. A. R. Taherian, P. Fustier and H. S. Ramaswamy, Int. J. Food Prop. 11, 24-43 (2008).

142. P. Walstra and A. L. de Roos, Foods Revs. Int. 9, 503-525 (1993).

143. D. M. A. Buzza, C.-Y. D. Lu and M. E. Cates, J. Phys. II France 5, 37-52 (1995).

144. Y. Hemar, R. Hocquart and F. Lequeux, J. Phys. II France 5, 1567-1576 (1995).

145. J. Benjamins, Wageningen University, 2000.

146. J. M. Franco, A. Guerrero and C. Gallegos, Rheol. Acta 34, 513-524 (1995).

147. A. Guerrero, P. Partal and C. Gallegos, J. Rheol. 42, 1375-1388 (1998).

148. D. J. McClements, Food emulsions: principle, practice and techniques. (CRC Press, Boca Raton, 1999).

149. S. L. Turgeon, C. Sanchez, S. F. Gauthier and P. Paquin, Int. Dairy J. 6, 645-658 (1996).

150. L. B. Famuso, M. Corredig and C. C. Akoh, J. American Oil Chemists' Soc. 78, 771$774(2001)$. 
151. C. Gallegos, M. Berjano and L. Choplin, J. Rheol. 36, 465-478 (1992).

152. C. Bower, C. Gallegos, M. R. Mackley and J. M. Madiedo, Rheol. Acta 38, 145-149 (1999).

153. J. M. Franco, P. Partal, D. Ruiz-Marquez, B. Conde and C. Gallegos, J. American Oil Chemists' Soc. 77, 975-983 (2000).

154. J. E. Moros, J. M. Franco and C. Gallegos, J. American Oil Chemists' Soc. 79, 837843 (2002).

155. B. P. Binks, J. H. Clint and C. P. Whitby, Langmuir 21, 5307-5316 (2005).

156. I. Masalova and A. Y. Malkin, Colloid J. 69, 185-197 (2007).

157. F. Pignon, A. Magnin and J.-M. Piau, J. Rheol. 40, 573-587 (1996).

158. A. J. Liu and S. R. Nagel, Nature 396, 21-22 (1998).

159. W. Losert, L. Bocquet, T. C. Lubensky and J. P. Gollub, Phys. Rev. Lett. 85, 14281431 (2000).

160. D. M. Mueth, G. F. Debregeas, D. F. Karczmar, P. J. Eng, S. R. Nagel and H. M. Jaeger, Nature 406, 385-389 (2000).

161. G. Debrégeas, H. Tabuteau and J.-M. di Meglio, Phys. Rev. Lett. 87, 178305-178308 (2001).

162. P. Coussot, J. S. Raynaud, F. Bertrand, P. Moucheront, J. P. Guilbaud and H. T. Huynh, Phys. Rev. Lett. 88, 218301-218304 (2002).

163. T. Gibaud, C. Barentin and S. Manneville, Phys. Rev. Lett. 101, 258302-258305 (2008).

164. S. A. Rogers, D. Vlassopoulos and P. T. Callaghan, Phys. Rev. Lett. 100, 128304128307 (2008).

165. A. Pouchelon, J. Meunier, D. Langevin, D. Chatenay and A. M. Cazabat, Chem. Phys. Lett. 76, 277-281 (1980).

166. K. Shinoda, H. Kunieda, T. Arai and H. Saijo, J. Phys. Chem. 88, 5126-5129 (1984).

167. F. O. Opawale and D. J. Burgess, J. Colloid Interface Sci. 197, 142-150 (1998). 


\section{Chapter 2}

\section{Experimental}

\subsection{Materials}

Na-caseinate powder (from bovine milk) was obtained from Sigma-Aldrich. The aqueous phase; a phosphate buffer (0.05 M, pH 6.8) was prepared using Milli-Q water, disodium hydrogen orthophosphate (BDH Chemicals, AnalaR grade, 99\%) and potassium dihydrogen orthophosphate (BDH Chemicals, AnalaR grade, 99\%), with $0.01 \mathrm{wt} \%$ sodium azide (SigmaAldrich, 99\%) added as an antimicrobial agent. All chemicals were used without further purification.

For the study on Na-caseinate dispersions, the dispersions of between 2 to $20 \mathrm{wt} \%$ were prepared at room temperature by dissolving the Na-caseinate powder in the aqueous phosphate buffer under continuous stirring. Stirring was ceased after the Na-caseinate powder was fully dissolved. This was checked via visual inspection. The lowest and the highest Na-caseinate concentration take about 15 minutes and 2 hours, respectively, to be fully dissolved. The lowest concentration of Na-caseinate dispersion appears to be near transparent. On further increasing Na-caseinate concentration, the dispersions become bluish and finally opaque. The samples were studied without any further $\mathrm{pH}$ adjustment.

For the study of emulsions in the concentrated region, i.e., 50 to $70 \mathrm{wt} \%$ oil, three systems have been investigated: tetradecane (Sigma-Aldrich, AnalaR grade, 99\%), commercial soybean oil (brand: Simply, distributor: Wilmar Trading Pte. Ltd.) and commercial palm oil (brand: Seri Murni, distributor: FFM Marketing Sdn. Bhd.). The Nacaseinate powder was pre-dissolved in the aqueous phase prior to adding oil. Since palm oil tends to solidify at room temperature, it is also pre-dissolved in a warm water bath $\left(\sim 50^{\circ} \mathrm{C}\right)$ before formulation. The oil and the Na-caseinate dispersion were mixed by hand before transferring to the jet homogeniser ${ }^{1}$. Emulsions $(15 \mathrm{~g})$ were prepared at a pressure of 5 bar 
and six to eight passthroughs. The resulting emulsions presented as visually homogeneous white suspensions.

All experiments were repeated to ensure reproducibility and a freshly prepared sample was used each new experiment unless otherwise specified. Hundreds of samples were prepared for each phase space determination. To probe the phase diagram of each of the three systems studied, macroscopic phase separation was monitored at room temperature as a function of time by sealing the emulsions in glass tubes of diameter $2.5 \mathrm{~cm}$. The samples were studied without $\mathrm{pH}$ adjustment. The variation of the height of the expelled aqueous phase relative to the total eight of the sample was monitored as a function of time.

\subsection{Emulsion Characterisation}

What follows is a discussion on the concepts of the four characterisation techniques that are salient to this thesis and are of interest to the author. The combination of these techniques allowed a more complete understanding of these complex systems. Laser diffraction particle sizing probes the droplet size distribution whereas nuclear magnetic resonance yields information about the dynamic nature of the emulsion. Rheology experiments were performed to determine the flow behaviour while cryo-SEM gives a time frozen picture of the emulsion microstructure. For further information, the interested reader is directed to the references that were used in compiling this section: [2-5] for laser diffraction particle sizing, [6-9] for nuclear magnetic resonance, [10-13] for rheology and [14, 15] for cryo-scanning electron microscopy.

\subsubsection{Laser Diffraction Particle Sizing}

\subsubsection{The Equivalent Sphere, and The Mean Particle Diameter}

In order to understand laser diffraction particle sizing, it is important to first know the concept of the equivalent sphere. The only shape that can be described by one unique number is the sphere. The question to ask then is how do we describe a three-dimensional object that is not a sphere, for example a cube, with only one number? This can be achieved by measuring some properties of the object (maximum length, minimum length, weight, 
volume or surface area, for example) and assuming that they refer to a sphere. As such a unique number can be derived to describe any object. For instance, using a balance we can measure the weight of a cube. This mass is then assumed to be that of an equivalent sphere ( $\left.4 / 3 \pi r^{3} \cdot \rho\right)$. From this, a unique number can be obtained by calculating the diameter of the equivalent sphere $(2 r)$ that has of same weight as the cube. This is called the equivalent sphere theory.

In the following section is discussed the parameters measured by laser diffraction particle sizing. Consider three spheres of diameter $x, y$ and $z$. The mean diameter appears to be the sum of all the diameters divided by the number of objects $(n)$ which is

$$
\frac{x+y+z}{3}=\frac{\Sigma d}{n} .
$$

The diameter terms in the numerator are all linear in $d$ (i.e., $d^{1}$ ) and there are no diameter terms in the denominator (i.e., $d^{0}$ ), this is denoted as $D[1,0]$.

Similarly, to compare these spheres on the basis of surface area, given that the surface area of a sphere is $\pi d^{2}$, we first sum up the square of each diameter, divide by $n$ and then take the square root to obtain a mean diameter. Therefore, the number-surface mean is

$$
\sqrt{\frac{\left(x^{2}+y^{2}+z^{2}\right)}{3}}=\frac{\Sigma d^{2}}{n}
$$

and is denoted as $D[2,0]$.

In laser diffraction, the particle size is often denoted as the sphere of equivalent surface area to the particle as $D[3,2]$ or the Sauter mean diameter:

$$
D[3,2]=\frac{x^{3}+y^{3}+z^{3}}{x^{2}+y^{2}+z^{2}} .
$$

If the density of the particles is constant, the technique can generate the equivalent numbervolume mean calculated as

$$
\sqrt[3]{\frac{\left(x^{3}+y^{3}+z^{3}\right)}{3}}=\frac{\sum d^{3}}{n}
$$

or the volume mean diameter denoted as $D[4,3]$ :

$$
D[4,3]=\frac{x^{4}+y^{4}+z^{4}}{x^{3}+y^{3}+z^{3}} .
$$

The absolute number of particles is less relevant than the sizes or the size distributions of the particles and it is not needed in order to calculate the mean diameter because $n$ does not appear in the numerator of the equation. There is also an infinite number of answers to the 
mean diameter because each technique is liable to generate different results. With laser diffraction particle sizing, a stable sample measured under recirculating conditions in a liquid suspension would have a volume mean reproducibility of $\pm 0.5 \%$. Conversion to a number mean yields an uncertainty of less than $1.0 \%$.

\subsubsection{Light Scattering}

In the context of particle sizing, "particles" means an aggregation of material whose refractive index $\left(n_{p}\right)$ differs from the refractive index of its surroundings $\left(n_{\text {med }}\right)$. The dipole reradiation patterns (primarily due to diffraction) arise from all the dipoles of such particles interfering both constructively and destructively with each other. Hence, particles "scatter" light in various directions with varying efficiency. Using an algorithm the pattern from a mixture of particle sizes is analysed (using the inherent software) to ascertain the particle sizes present.

A given particle size distribution corresponds to a single scattered pattern. Different wavelengths are scattered by the particle size distribution differently. Fig. 2.1 is a diagram showing the collection of the diffracted light which is subsequently focused onto the detector where electrical signals result. The detectors also respond to the amount of light (intensity) reaching them, which is directly related to the number of particles present having a particular size. Use of a single wavelength source of laser light is essential. This avoids "connecting points" between distributions that develop when different incident wavelengths of light are used.

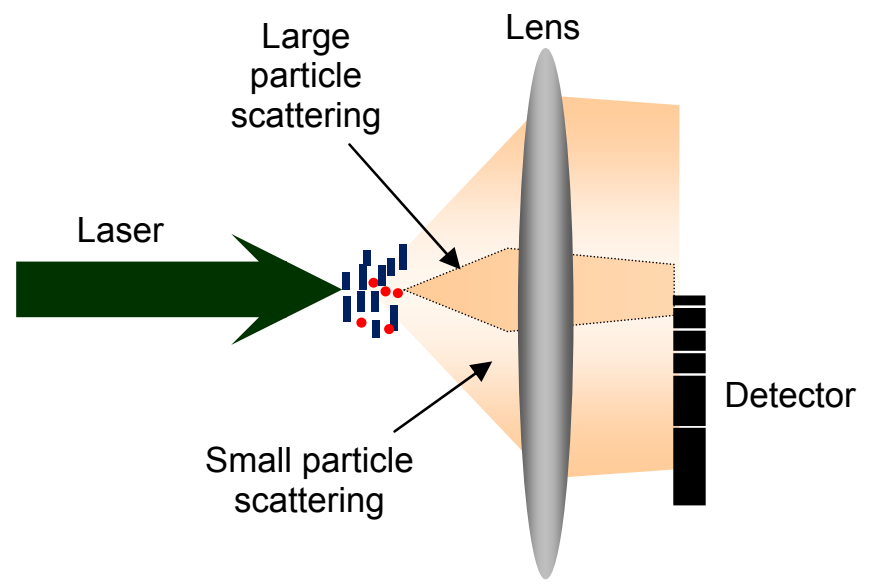

Figure 2.1: Diagram showing the concept of light scattering. 


\subsubsection{Mie Theory}

Mie theory, also called Lorenz-Mie theory or Lorenz-Mie-Debye theory is not considered an independent theory but an analytical solution of Maxwell's equations for the multiple scattering of electromagnetic radiation by polydisperse particles (also known as Mie scattering). Although this theory is based on homogeneous spherical particles of any size, it is also applied to non-spherical particles.

Mie theory introduces two terms, $n_{x}$ and $x$. The magnitude of the refractive index mismatch between the particle and the medium $n_{x}$, is expressed as:

$$
n_{x}=\frac{n_{p}}{n_{\text {med }}} .
$$

The size of the surface of refractive index mismatch is expressed as a size parameter $x$ where $2 \pi a$ is the meridional circumference of the sphere ( $a=$ radius of the particle) and $\frac{\lambda}{n_{\text {med }}}$ is the wavelength of light in the medium:

$$
x=\frac{2 \pi a}{\frac{\lambda}{n_{\text {med }}}} .
$$

A Mie theory calculation yields a dimensionless scattering efficiency parameter, $Q_{s}$ which relates the cross-sectional scattering area, $\sigma_{s}\left(\mathrm{~cm}^{2}\right)$, to the true geometrical cross-sectional area of the particle, $A=\pi a^{2}\left(\mathrm{~cm}^{2}\right)$ :

$$
Q_{s}=\frac{\sigma_{s}}{A} .
$$

Particle size distribution can be obtained by fitting the raw data using Mie theory.

As light scattering experiment requires extensive dilution and stirring, this results in the dissociation of weakly held three-dimensional structures. Thus, this technique only probes the base droplet/aggregate unit in the system studied and not any larger aggregates held together weakly. 


\subsubsection{Experimental Parameters}

Particle size distributions and apparent hydrodynamic radii of Na-caseinate aggregate dispersions and emulsion droplets were determined using a MALVERN Mastersizer 2000 with a $45 \mathrm{~mm}$ lens and a small sample dispersion unit, where the pump and stir component are manually controlled. A He-Ne laser is used as the incident light source with wavelengths of $633 \mathrm{~nm}$ and $452 \mathrm{~nm}$, covering a size range from 0.02 to $2000 \mu \mathrm{m}$.

The refractive indices for Na-caseinate dispersions were identified to be between 1.54 to 1.56 for the dispersions and 1.46 to 1.48 for the dispersant. For emulsions, the refractive indices were first obtained using an Abbé-refractometer. These values were used as the initial estimate in obtaining the scattering data. The model used in the Mastersizer software to calculate the particle diameter is Mie theory, assuming the sample is comprised of polydisperse irregular particles. The refractive indices were determined to be between 1.45 to 1.52 for the emulsions and 1.33 for the dispersant. An adsorption value of 0.001 was used for all samples. Variation of this adsorption value did not significantly alter the obtained distributions.

\subsubsection{Nuclear Magnetic Resonance (NMR)}

\subsubsection{Nuclear Spin and Zeeman Interaction}

All nucleons, that is protons and neutrons, have intrinsic angular momentum called spin. Both protons and neutrons have spin $\hbar / 2$, where $\hbar$ is the reduced Planck's constant $(h)$. Under a system with $\hbar=1$, protons and neutrons will have spin of $-1 / 2$. The overall spin of a nucleus is contributed to by the individual spins of the nucleons and is characterised by the spin quantum number $S$. The magnetic dipole moment of a nucleus, $\mu$, is defined as:

$$
\mu=\gamma I
$$

where $I$ is the angular momentum and $\gamma$ is the gyromagnetic ratio $\left(2.675 \times 10^{8} \mathrm{rad} \mathrm{s}^{-1} \mathrm{~T}^{-1}\right.$ for a proton).

Nuclear magnetic resonance is a phenomenon where an interaction between the magnetic nuclei and the magnetic field occurs. Consider nuclei with $S \neq 0$, say $S=1 / 2$, e.g., 
${ }^{1} \mathrm{H},{ }^{3} \mathrm{H},{ }^{13} \mathrm{C},{ }^{15} \mathrm{~N},{ }^{19} \mathrm{~F}$ or ${ }^{31} \mathrm{P}$. The nucleus has two possible spin states: $m=1 / 2$ or $m=-1 / 2$ (also referred to as spin-up and spin-down). These spin states, in the absence of an external magnetic field, are degenerate, i.e., they have the same energy. When the nucleus is placed in a magnetic field, $B_{0}$ (usually aligned along the laboratory $z$-axis), the interaction between the nuclear magnetic moment and the external magnetic field results in the two spin states no longer being degenerate. The interaction energy is then given by the Hamiltonian

$$
H_{z}=-\mu \cdot B_{0} .
$$

This form of the Hamiltonian is known as the Zeeman interaction. The energy difference between the two spin states is:

$$
\Delta E=\gamma \hbar B_{0}
$$

Transition from the lower energy state to the higher energy state, as shown in Fig. 2.2, happens when photons with energy, $E_{\text {photon }}=h v$, matching the energy difference between the two spin states, are absorbed. Here, $h$ is Planck's constant and $v$ is the resonance frequency (typically corresponding to the radio-frequency (or r.f.) range of the electromagnetic spectrum for most spin transitions utilised in NMR experiments) required for the transition;

$$
v=\frac{\omega_{0}}{2 \pi}
$$

where $\omega_{0}$ is the Larmor precession frequency. It is this magnetic resonant absorption which is detected in NMR.

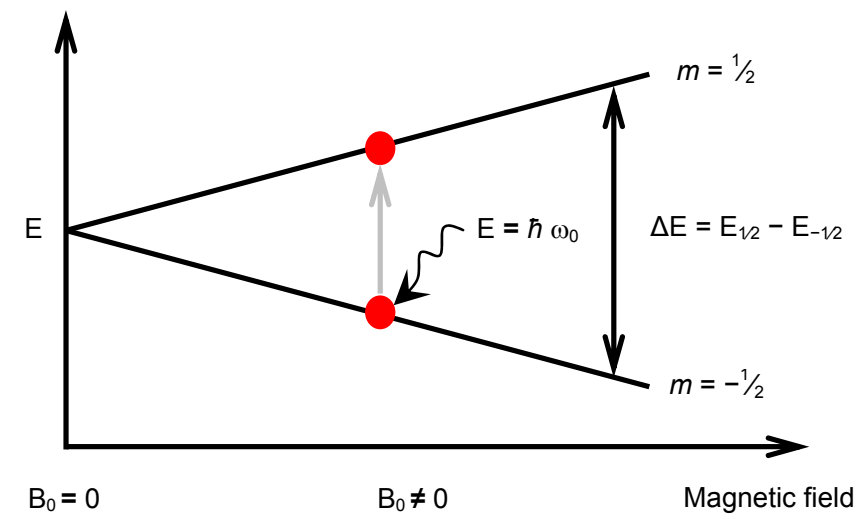

Figure 2.2: Two possible spin states of a spin $1 / 2$ system. Transition from the lower energy state to the higher energy state requires the absorption of photons at the Larmor frequency, $\omega_{0}$. 


\subsubsection{Pulses and Signal Detection}

The perturbation of spins from their equilibrium states can be achieved via the application of a pulse of r.f. radiation resulting in the absorption of Larmor frequency photons. The magnitude of that linearly polarised oscillating magnetic (r.f.) field is denoted as $B_{1}$ and its orientation is transverse to $B_{0}$. In Fig. 2.3 is illustrated the precession of the spin magnetisation vector $\mathrm{M}$ under the influence of both $B_{0}$ and $B_{1}$.

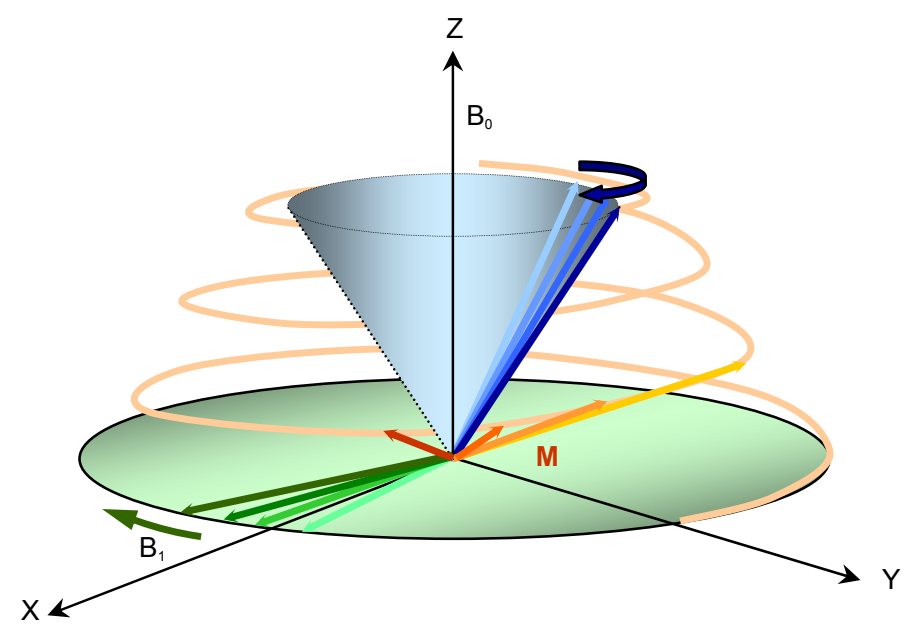

Figure 2.3: The precession of the spin magnetisation vector $\mathrm{M}$ in the presence of both $B_{0}$ and $B_{1}$. The figure also illustrates the individual magnetisation vector that precesses about the $z$-axis in the presence of a static magnetic field $B_{0}$ at the Larmor frequency $\left(M_{z}\right.$, shown in blue) and an oscillating transverse field $B_{1}\left(M_{x, y}\right.$, shown in green).

To simplify the following discussion, the $B_{1}$ field has been arbitrary chosen to lie along the $x$-axis. The r.f. radiation pulse that rotates the angle of magnetisation vector about the $x$ axis through $90^{\circ}$ is known as a $90_{x}$ pulse. Similarly, when a $180_{x}$ pulse is applied, the magnetisation is inverted and oriented along the $z$-axis. The frequency, duration, amplitude and shape of the pulse determine which spins are perturbed. The bandwidth of a pulse of duration $t_{p}$ is in the order of $2 t_{p}^{-1}$.

A set of r.f. pulses and time delays allowing examination of the sample specific behaviour is called a pulse sequence. A typical pulse sequence can be divided into several sections; preparation, mixing and acquisition times. As the name suggests, in the preparation section the spin system is being prepared. The incorporation of a mixing time into the pulse 
sequence is to allow mixing of density matrix terms. This part of the sequence often ends with a 'read' pulse which allows the state of the spin system to be read and stored. The acquisition time is when signal from the spin system is being recorded.

The following discussion focuses on the two relaxation modes that contribute to the recovery of spins to their equilibrium states after the application of a r.f. pulse, i.e., spinlattice relaxation, known as $T_{1}$ and spin-spin relaxation, known as $T_{2}$.

Nuclei held within a lattice structure are in constant vibrational and rotational motion. The application of a r.f. pulse causes an increase in the vibrational and rotational motion of the nuclei. In time, the equilibrium state of excited nuclei will be restored via the dissipation of energy gained from the r.f. pulse to the surrounding lattice. Thus, $T_{1}$ refers to the time for the preceding process to occur. In other words, $T_{1}$ characterises the rate at which the longitudinal component of the magnetisation vector, $M_{z}$ recovers. Transverse magnetisation is represented by phase coherence between spin states as illustrated in Fig. 2.3. Spin-spin relaxation refers to the time where the excited nuclei reach their equilibrium state through reduction of the transverse magnetisation. In other words, $T_{2}$ characterises the rate at which the $M_{x, y}$ component of the magnetisation vector decays in the transverse magnetic plane. A combination of energy exchange with the lattice in the transverse magnetic plane and other terms that leads to the loss of transverse magnetisation, results in $T_{2} \leq T_{1}$. In addition to $T_{2}$, $T_{2}^{*}$ is used to describe the increase in the rate of transverse relaxation caused by magnetic field inhomogeneities.

Following the application of a r.f. pulse, the primary NMR signal is detected in the time-domain as a decaying oscillating signal induced by the free precession of spins (which causes a non-zero transverse magnetisation). The observable signal is usually called the free induction decay (FID). This time-domain signal is discretely sampled and then Fourier transformed to obtain a spectrum in the frequency-domain as shown in Fig. 2.4. 

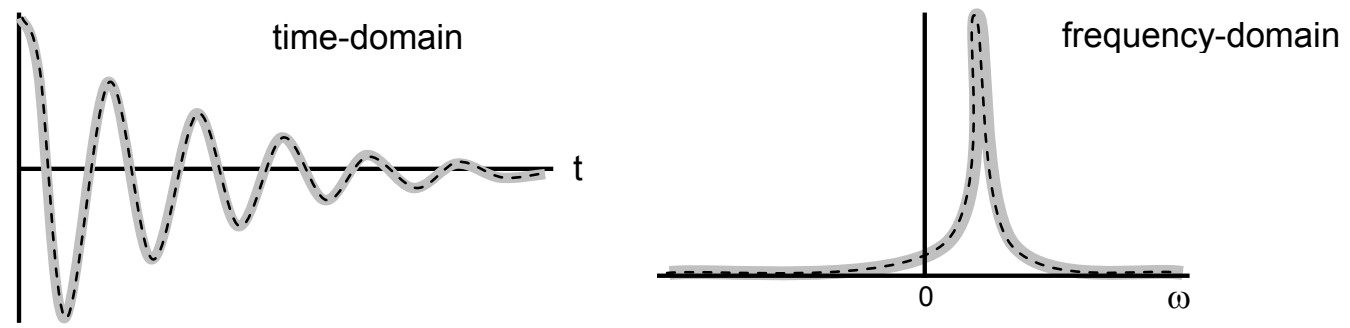

Figure 2.4: Free induction decay (FID) obtained via the sampling of $N$ discrete points (black dashes) over time. The continuous nature of the acquisition process is hinted by the grey line. Fourier transformation of the timedomain signal yields a spectrum.

Noise caused by the Brownian motion of electrons and artefacts that arise from imperfections in the r.f. pulses and receiver coil are the two unwanted components that produce a low signal-to-noise ratio. The effect of noise can be reduced by adding the signal from $N$ experiments, whereas 'phase cycling', a periodic change of the phase of the applied $B_{1}$ field can be employed to diminish or eliminate artefacts.

In the following section, we introduce the methods of measuring nuclear relaxation and the pulse sequences used in this thesis.

\subsubsection{Relaxation Measurements}

\subsection{Saturation Recovery}

$T_{1}$ measurement can be performed via the saturation recovery method illustrated in Fig. 2.5. The pulse sequence consists of multiple $90^{\circ}$ pulses with a decreasing delay or relatively short repetition time $(\tau)$ between the pulses. Residual longitudinal magnetisation after the first $90^{\circ}$ pulse is dephased by a gradient pulse. The newly developed longitudinal magnetisation, after the application of the dephasing gradient, is rotated into the transverse plane by another $90^{\circ}$ pulse. The last application of a $90^{\circ}$ pulse is followed by an echo. The spin system is said to be in a saturated state when the first $90^{\circ}$ pulse is applied which tilts the magnetisation into the xy-plane. The $M_{z}$ magnetisation recovers according to the formula derived from the Bloch equations:

$$
M_{z}=M_{z_{0}}\left(1-\exp \left(\frac{-\tau}{T_{1}}\right)\right) .
$$


If the chosen repetition time, $\tau$, is smaller than $T_{1}$, the full magnetisation $M_{z_{0}}$ is not recovered before the next $90^{\circ}$ pulse is applied (the bracketed expression is still less than 1 ). An example of a $T_{1}$ relaxation curve is shown in Fig. 2.6.

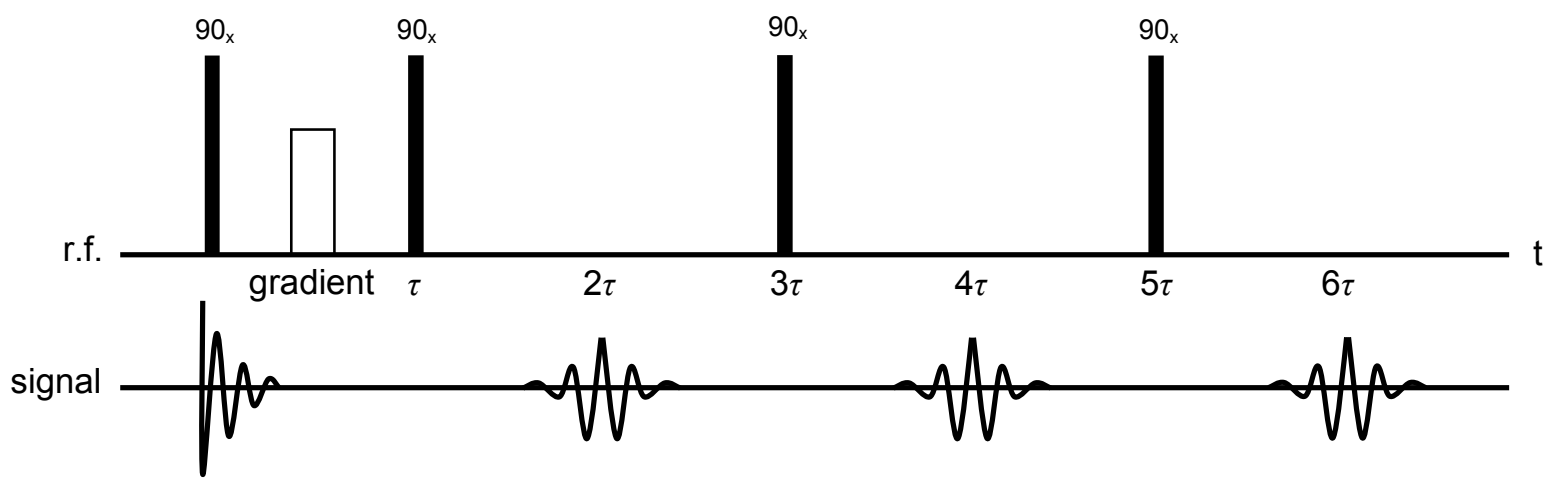

Figure 2.5: The saturation recovery pulse sequence for $T_{1}$ measurement. Following a repeating $90_{x}$ pulse sequence at times $(2 n) \tau$, spin echoes are formed.

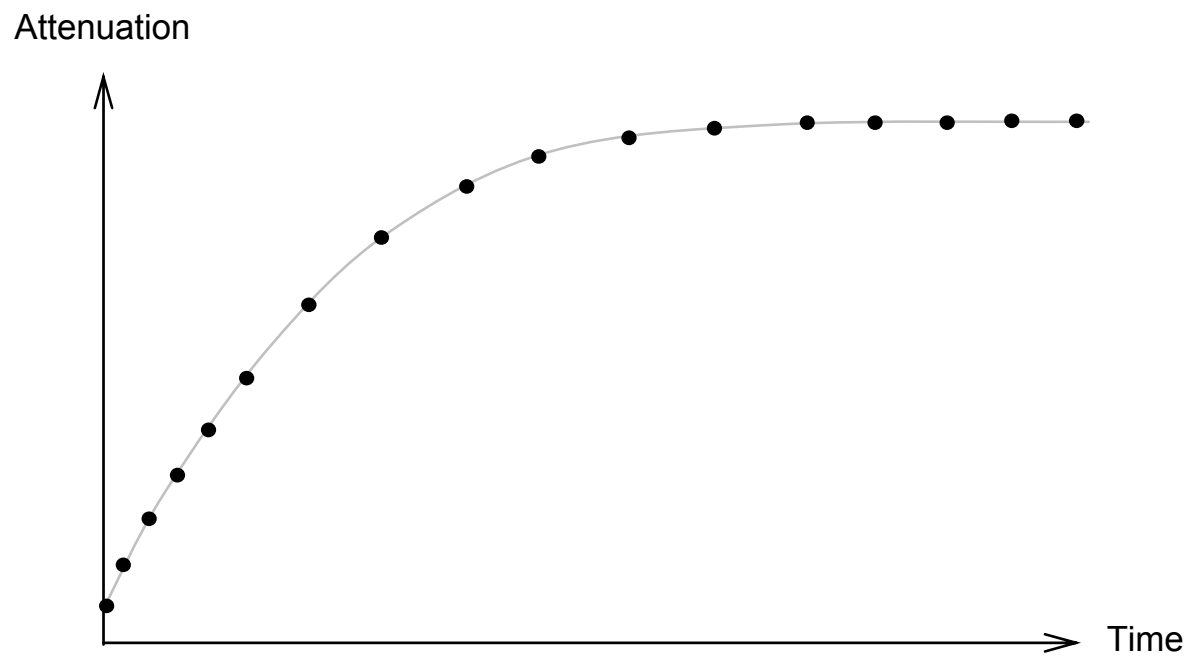

Figure 2.6: Representation of a $T_{1}$ relaxation curve.

\subsection{Carr-Purcell-Meiboom-Gill (CPMG)}

$T_{2}$ measurement was first suggested by Carr and Purcell ${ }^{16}$. It consists of the initial $90_{x}$ pulse followed by repeating $180_{x}$ pulses. However, due to the r.f. inhomogeneities, it creates accumulated phase errors in the $180_{x}$ pulses. This problem was solved by Meiboom and Gill ${ }^{17}$. They suggested that the $180^{\circ}$ pulses have a different phase from the initial $90^{\circ}$ pulse 
so that any phase errors from one pulse are cancelled by the following pulse and echoes that are formed at delay times $2 \tau, 4 \tau,(2 n) \tau$ have the same sign. An illustration of the CPMG sequence with an example of a $T_{2}$ relaxation curve is shown in Fig. 2.7 and Fig. 2.8, respectively.

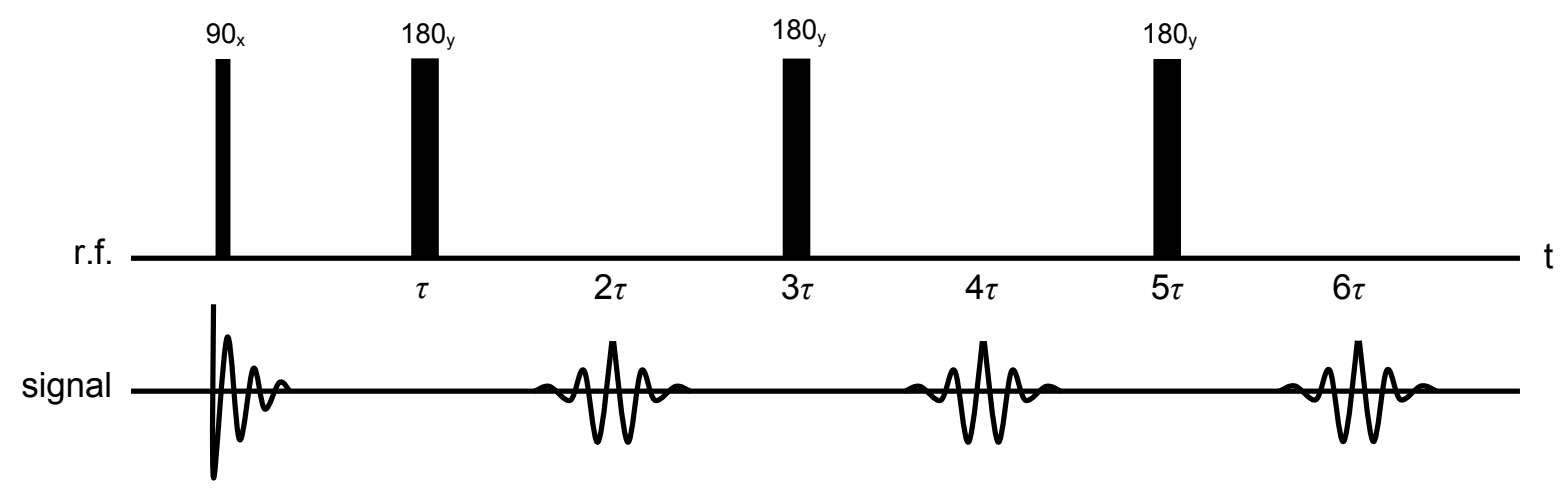

Figure 2.7: The Carr-Purcell-Meiboom-Gill pulse sequence for $T_{2}$ measurement. Following a repeating $180_{y}$ pulses at times $(2 n) \tau$, spin echoes are formed.

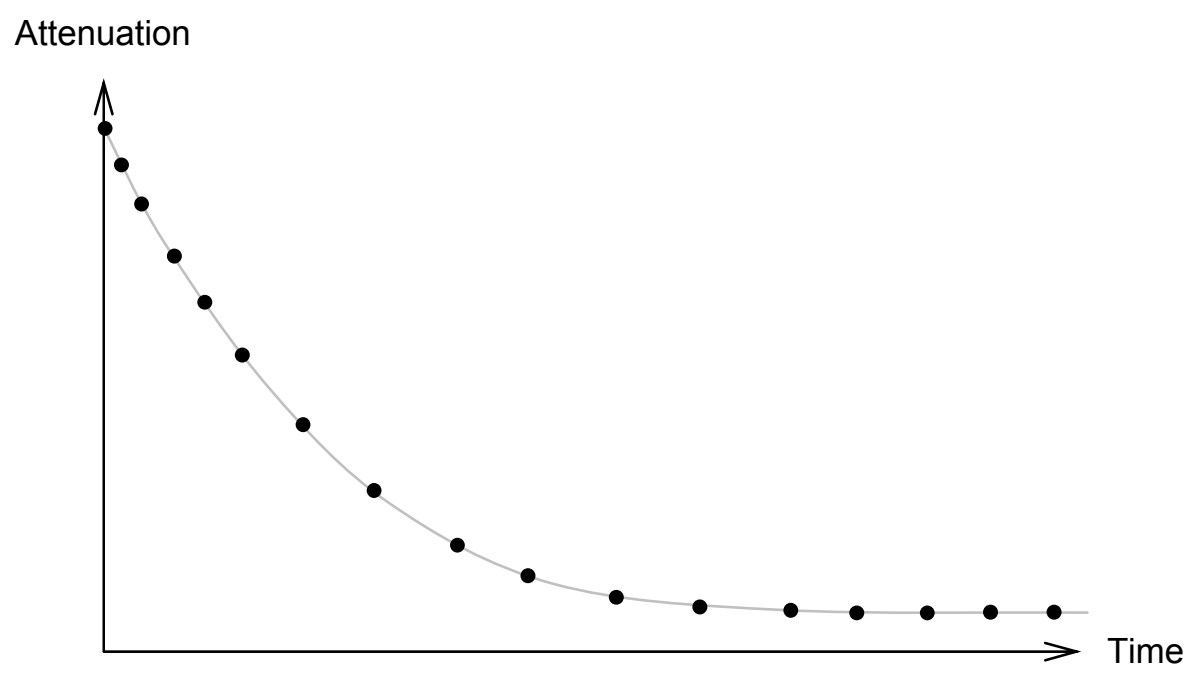

Figure 2.8: A representation of $T_{2}$ relaxation curve.

\subsubsection{Pulsed Gradient Stimulated Echo (PGSTE) NMR}

The translational motion of molecules in solution, or the so-called self-diffusion can be monitored using the pulsed gradient stimulated echo sequence (PGSTE), implemented by Stejskal and Tanner ${ }^{18}$. The PGSTE pulse sequence is shown in Fig. 2.9. Initially, a 90, radio-frequency pulse is applied, which rotates the magnetisation from the $z$-axis into the xy- 
plane. The spins subsequently begin to dephase due to $T_{2}$ relaxation. This is followed by a preparation interval where the first gradient pulse spatially encodes the position of each nuclear spin. Another $90_{x}$ radio-frequency pulse is applied to rotate the spins into the xzplane. The spins at this stage are subjected only to $T_{1}$ relaxation. The third $90_{x}$ pulse rotates the spins back into the xy-plane again. This is followed by a read interval where the spins spatial distribution can be decoded by applying the second gradient pulse. The spins that have moved during the waiting time between the preparation and read intervals will result in an incomplete phase reversal. The summation of the accumulated phases leads to a net magnetisation change and an attenuation of the NMR signal. This echo attenuation is dependent on $g, \delta, \Delta$ and the diffusion coefficient ${ }^{19}$ of the molecules under study.

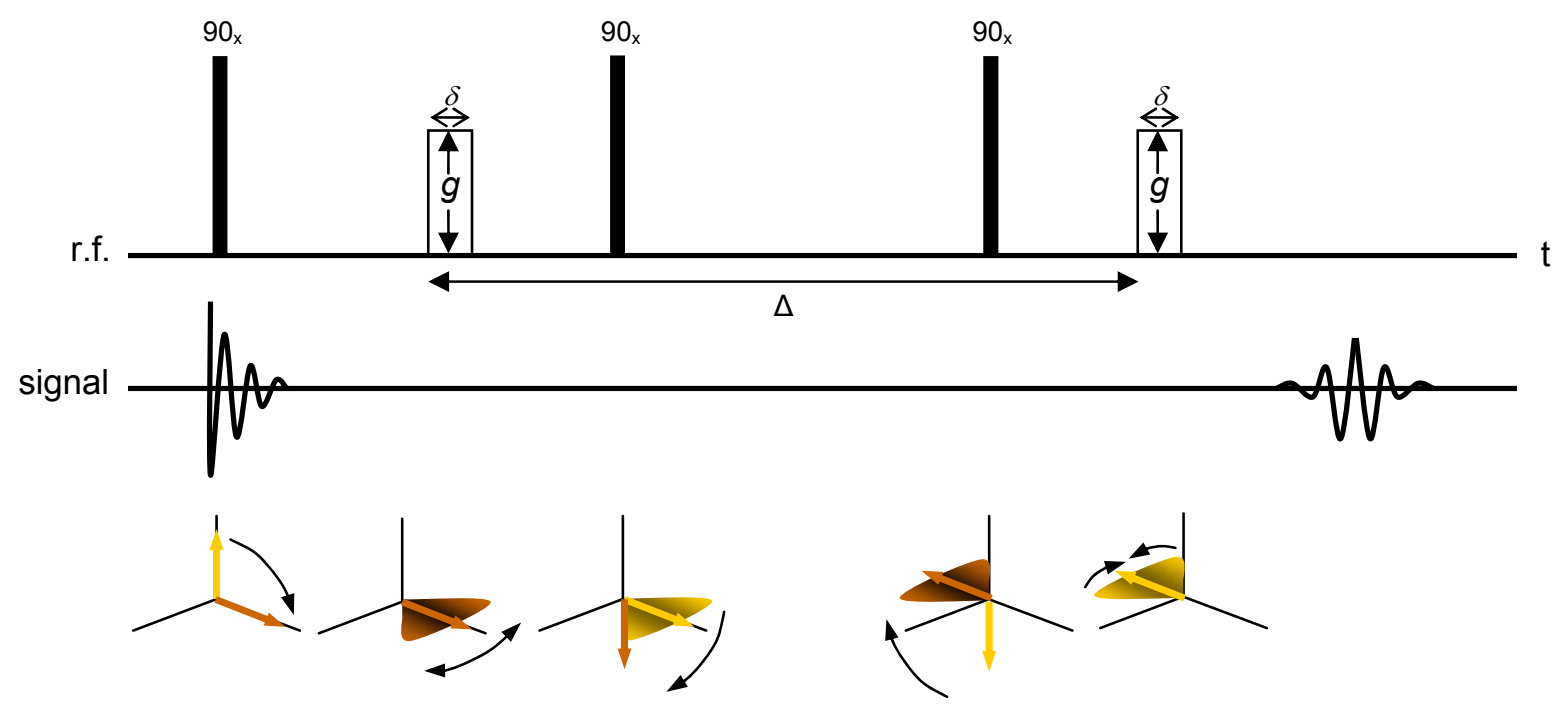

Figure 2.9: The pulsed gradient stimulated echo experiment. The pulse sequence starts with a $90_{x}$ pulse which rotates the spins into the xy-plane followed by a spin dephase. The second $90_{x}$ pulse rotates the dephased spins into the xz-plane. The spins are then brought back to the transverse plane via the application of third $90_{x}$ pulse. The two gradient pulses encode and decode the positions of each perturbed spin. $g$ and $\delta$ are the amplitude and length of the magnetic field gradient pulses. $\Delta$ is the time between the leading edges of the gradient pulses. Incomplete phase reversal yields an echo attenuation.

The molecular displacements can be probed over a wide range of time-scales by varying the time between the two gradient pulses. The longest diffusion time that can be observed is limited by the relaxation times of the sample. The shortest time accessible is set by instrumental limitation, i.e., the difficulty of applying strong and matched magnetic field gradient pulses without generating eddy currents. The diffusion coefficient, $D$, can be 
directly estimated from analysis of the PGSTE-NMR data using the equation developed by Stejskal and Tanner ${ }^{18}$ :

$$
\ln \frac{E}{E_{0}}=-\gamma^{2} g^{2} \delta^{2}\left(\Delta-\frac{\delta}{3}\right) D
$$

where $E$ is the attenuation, $E_{0}$ is the attenuation without an applied gradient, $\gamma$ is the magnetogyric ratio of the proton, $g$ is the strength of the gradient probe, $\delta$ is the duration of the gradient pulse and $\Delta$ is the time between the two gradient pulses.

If molecules or objects diffuse with no boundary or obstruction effects they are said to be in a state of unrestricted diffusion. Unrestricted diffusion occurs when $\Delta$, the experimental observation time, is small. That is the displacement of the molecule or object is smaller than the characteristic length scale of the system. Taking the case of diffusion within a droplet, molecules do not on average encounter the walls of the droplet during the measurement time. As such the molecular self-diffusion coefficient (the unrestricted diffusion) is measured. As $\Delta$ is increased, the total displacement of the molecule becomes larger than the characteristic length scale of the system. In the case of a molecule diffusing inside a droplet, we now have the case where the droplet walls are on average encountered by the molecule and the molecular diffusion is said to be restricted. A diffusion coefficient slower than the unrestricted diffusion will be measured. In the restricted diffusion regime, the mean displacement of the molecule is no longer dependent on $\Delta$, but rather on the characteristic length scale of the system. As NMR is a volume-based technique, it is often blind to the smallest aggregates/droplets in a system studied since they make only a very small contribution to the system on a volume basis.

\subsection{Experimental Parameters}

Samples were housed in $5 \mathrm{~mm}$ NMR tubes which were fixed in a diffusion probe of a $9.4 \mathrm{~T}$ superconducting magnet equipped with a Bruker Avance-460 NMR system. The temperature of the sample was controlled at $25^{\circ} \mathrm{C}$ by a temperature control unit consisting of a heating element working in conjunction with a constant gas flow. Calibration was performed using Milli-Q water with a known diffusion coefficient ${ }^{20}$ of $2.3 \times 10^{-9} \mathrm{~m}^{2} \mathrm{~s}^{-1}$.

The following is the parameter settings for the study of Na-caseinate dispersions. For water self-diffusion measurements, the values of $\Delta$ and $\delta$ ranged from 20 to $200 \mathrm{~ms}$ and from 1.8 to $2.3 \mathrm{~ms}$, respectively with a maximum gradient of $2 \mathrm{~T} \mathrm{~m}^{-1}$. For casein aggregates 
self-diffusion measurements, $g, \Delta$ and $\delta$ values ranged from 4 to $9 \mathrm{~T} \mathrm{~m}^{-1}$, from 12 to $140 \mathrm{~ms}$ and from 2 to $4 \mathrm{~ms}$, respectively. A total of 64 scans and 64 data points were collected with a recycling delay of $3 \mathrm{~s}$.

The following is the parameter settings for the study of emulsions. For water selfdiffusion measurements, the values of $\Delta$ and $\delta$ were 20 to $200 \mathrm{~ms}$ and $2 \mathrm{~ms}$, respectively with a maximum gradient of $2 \mathrm{~T} \mathrm{~m}^{-1}$. For oil self-diffusion measurements, $\Delta, \delta$ and $g$ values ranged from 40 to $2200 \mathrm{~ms}$, from 2 to $6 \mathrm{~ms}$ and from 2 to $9 \mathrm{~T} \mathrm{~m}^{-1}$, respectively. A total of 8 scans and 64 data points were collected with a recycling delay of $3 \mathrm{~s}$.

\subsubsection{Inverse Laplace Transformation}

Many measured NMR signals are in the form of a decaying exponential. The inverse Laplace transform is a method that converts the decaying exponential signals into a distribution of properties in materials characterisation such as characteristic times. Taking the example of a monoexponential decay, the measured signal $M$ is given by

$$
M(t)=\exp \left(\frac{-t}{\tau}\right)
$$

where $t$ is the experimental time and $\tau$ is the characteristic time. As seen in Fig. 2.10, short characteristic times produce fast exponential decays and vice versa. The characteristic times for multiexponential decays can also be retrieved by this transformation. Because real measurements contain noise, small changes in the measured data will lead to large variations in the resulting transformation. To address the problem, in the next section we will concisely discuss the regularisation method that we use in processing our data.
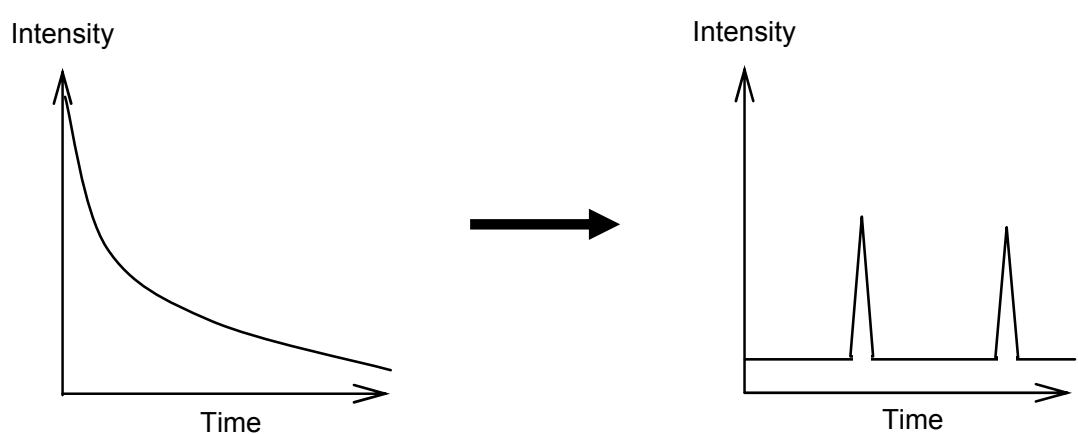

Figure 2.10: Schematic exponential decays transformed by a 1D inverse Laplace algorithm. 


\subsection{Regularisation}

Regularisation as the name suggests, is a process which associates with making a function more 'regular' or smooth so that it forms a unique solution and is less sensitive to noise. The non-negative least squares (NNLS) algorithm ${ }^{21}$ can be viewed as a simple form of regularisation often used as part of the inverse Laplace transform.

Consider an ill-posed system $M=F K$. We can write a function

$$
\chi^{2}=\|F K-M\|^{2}+\alpha\|L F\|^{2}
$$

where the variables are in the real domain: $F$ is the probability density $\left(F \varepsilon \mathfrak{R}^{n}\right), K$ is the kernel $\left(\begin{array}{lll}K & \varepsilon & \mathfrak{R}^{m \times n}\end{array}\right)$ and $M$ is the measured data $\left(M \varepsilon \mathfrak{R}^{m}\right) . m$ and $n$ are the dimensions of

the column vector. $\|\cdot\|$ indicates the sum of the matrix elements squared, $\alpha$ indicates the regularisation factor which regulates the amount of smoothing applied to the inversion and $L$ indicates the regularisation method. NNLS solves the matrix equation algebraically, but subject to the added constraint that the solution $F$ contains no negative elements. The idea of least squares is to best fit a data set by adjusting the parameters of the model function used so that the sum of squared residuals has its least value with a residual being the difference between a measured value and the calculated value given by the model.

The optimal value of $\alpha$ is usually chosen to minimise $\chi^{2}$. In Fig. 2.11 is shown the variation of $\chi^{2}$ plotted as a function of $\alpha$. The correct $\alpha$ value is at the start of the plateau. This value is used for an inversion as it is the least sensitive to random noise. $\alpha$ values that are smaller than this value are over smoothed while larger than this value are under smoothed.

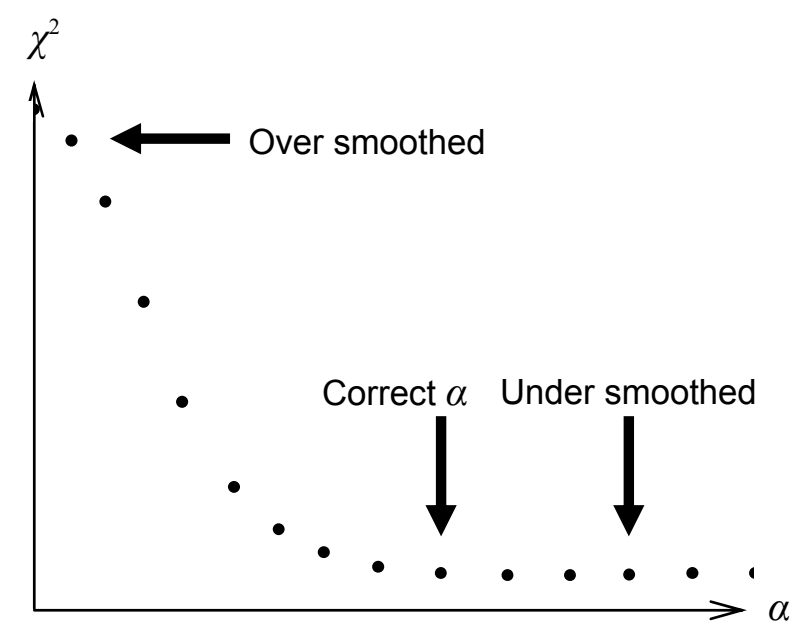

Figure 2.11: The optimisation of $\alpha$ value for an inversion obtained by performing a $\chi^{2}$ analysis to the data set. 


\subsubsection{Imaging}

In this section we present a brief discussion on the fundamental relationship of NMR imaging. Recalling section 2.2.2.2 where the definition of pulses is introduced, 'soft' pulses are used in NMR imaging instead to selectively excite part of the spins in the sample. A soft pulse is a sinc-modulated pulse and works in conjunction with a magnetic field gradient. A gradient with the magnitude, $G_{z}=\frac{\Delta \omega}{\gamma \Delta z}$ and a soft pulse of bandwidth $\Delta \omega$ are needed in order to excite a band of spins with physical width $\Delta z$. In Fig. 2.12 is shown the truncated one-lobe sinc function and its response in the frequency-domain.

(a)

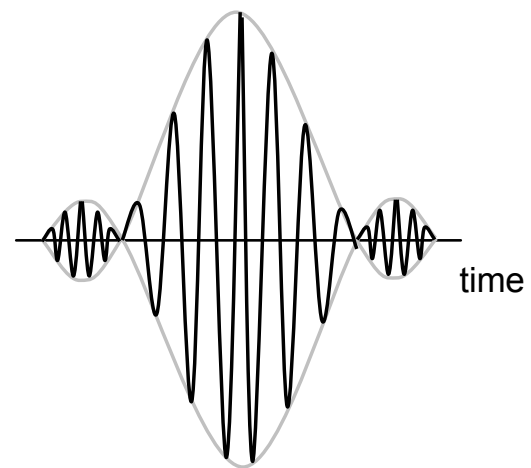

(b)

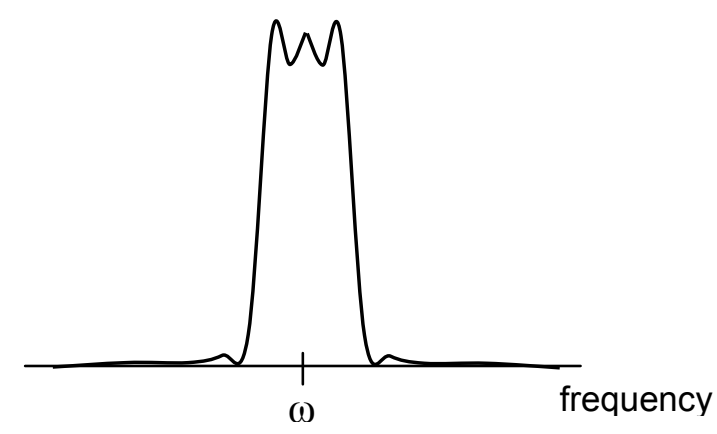

Figure 2.12: A 'soft' pulse used for slice selection (a) in the time-domain with truncated one-lobe sinc function and (b) in the frequency-domain with a rectangular hat function.

In the presence of a magnetic field gradient, $G=\operatorname{grad} B_{0}$, the local Larmor frequency of a precessing spin is

$$
\omega(r)=\gamma B_{0}+\gamma G \cdot r
$$

where $r$ is the vector representing the coordinate of the spin. Consider $\rho(r) d V$ spins, a group of spins at a point $r$ occupying a volume $d V$ where $\rho(r)$ is the nuclear spin density. The signal obtained from this volume is given by

$$
d S(G, t) \propto \rho(r) d V \exp [i \omega(r) t]=\rho(r) d V \exp \left[i\left(\gamma B_{0}+\gamma G \cdot r\right) t\right] .
$$

The spins are "labelled" according to their position in space by the magnetic field gradient. The NMR signal is then expressed as

$$
S(k)=\int \rho(r) \exp (i 2 \pi k \cdot r) d r
$$


where $d r$ is now the volume element. $k$ is the reciprocal space vector conjugated to $r$ :

$$
k=(2 \pi)^{-1} \gamma G t
$$

with $t$ being the evolution time of the spins in the presence of the magnetic field gradient. The inverse Fourier transformation of $S(k)$ gives the spin density as

$$
\rho(r)=\int S(k) \exp (-i 2 \pi k \cdot r) d k .
$$

While signal acquisition in $k$-space allows the imaging of spin density, signal acquisition in $q$-space allows the imaging of the average propagator. Referring to the pulsed gradient stimulated echo experiment shown in Fig. 2.9, the two gradient pulses with a duration $\delta$ and amplitude $g$, separated by the time delay $\Delta$, are applied, imparting a phase shift to the spin isochromat which depends directly on the motion of the spins. If the average propagator of any spin which has moved $R$, i.e., from $r$ to $r^{\prime}$ over the observation time $\Delta$ is $P_{s}(R, \Delta)$ then the echo signal has the form

$$
S(g, \delta, \Delta)=\int \rho(r) \int P_{s}(R, \Delta) \exp (i 2 \pi q \cdot R) d R
$$

where $q$ is a second reciprocal space vector defined by

$$
q=(2 \pi)^{-1} \gamma \delta g .
$$

The signal can now be expressed both in $k$ - and $q$-space as

$$
S(k, q)=\int \rho(r)\left(\int P_{s}(R, \Delta) \exp (i 2 \pi q \cdot R) d R\right) \times \exp (i 2 \pi k \cdot r) d r .
$$

A velocity map can be obtained by performing a double inverse Fourier transformation with respect to $k$ - and $q$-space.

\subsubsection{NMR Velocimetry}

NMR velocimetry ${ }^{8}$ can be conveniently divided into two parts; velocity imaging and phase encoding using PGSTE, as shown in the pulse sequence in Fig. 2.13. In the velocity encoding part, two gradient pulses of duration $\delta$ and amplitude $g$, separated by the time delay $\Delta$, are applied. The first gradient pulse spatially encodes the position of each nuclear spin and the second gradient pulse decodes this spatial distribution. In the imaging part, the first soft pulse is applied with a magnetic field gradient in the $y$-direction to selectively excite spins in a slice of thickness $2 \mathrm{~mm}$ whereas the second soft pulse works in conjunction with another magnetic gradient in the $z$-direction to selectively excite spins in a slice of thickness $20 \mathrm{~mm}$ transverse to the first slice. 

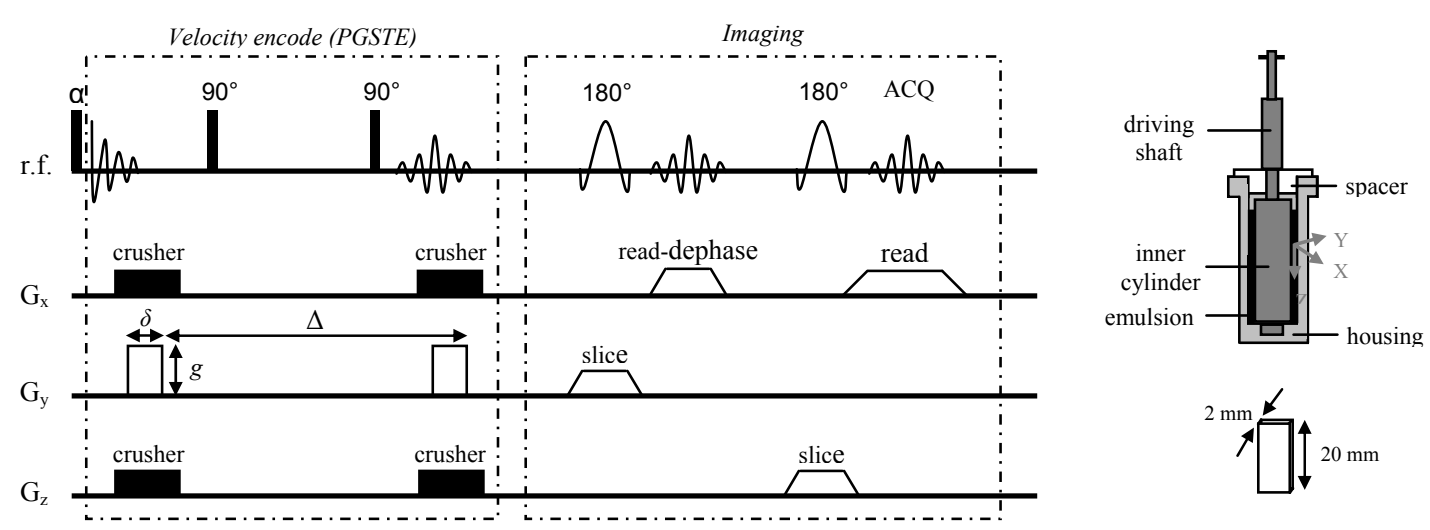

Figure 2.13: NMR velocity imaging pulse sequence and schematic of the Couette geometry used for the NMR velocimetry experiments. Phase encoding is along the $y$-axis. The crusher gradients in the $x$ - and $z$-directions suppress any signal contribution from the hard r.f. pulses ${ }^{22}$.

\subsection{Experimental Parameters}

NMR velocimetry measurements were performed using a cylindrical Couette geometry machined from polyether ether ketone (PEEK). The inner cylinder acting as the rotor has an o.d. of $17 \mathrm{~mm}$ and a hatched surface, and the outer cylinder has an i.d. of $19 \mathrm{~mm}$, creating a Couette gap width of $1 \mathrm{~mm}$. Approximately $3 \mathrm{~mL}$ of the concentrated emulsion is transferred to the gap of the Couette geometry and the inner cylinder is filled with $0.1 \mathrm{wt} \% \mathrm{CuSO}_{4}$ as a marker fluid. The Couette is fitted inside a $25 \mathrm{~mm} \mathrm{H}-1$ tuned (c.a. $400.1 \mathrm{MHz}$ ) r.f. coil and fixed to a Bruker Micro2.5 probe. The NMR velocimetry measurements were performed using a 9.4 $\mathrm{T}$ superconducting magnet equipped with a Bruker Avance-400 NMR spectrometer. The inner cylinder of the Couette was rotated by stepping the shear rate from 0.08 to $6.41 \mathrm{~s}^{-1}$ with increments of 10 steps per decade and a dwell time of 6 minutes at each shear rate. Initially, the fresh sample is left to age for approximately $2 \mathrm{hrs}$ in the Couette geometry. The temperature of the sample is maintained at $25^{\circ} \mathrm{C}$ by a temperature control unit that consists of a heating element working in conjunction with a constant gas flow.

NMR velocimetry measurements were performed using volume-selective onedimensional imaging with the signal encoded for velocity using a PGSTE sequence, as shown in Fig. $2.13^{8}$. A flip angle, $\alpha$, of $\sim 45^{\circ}(20 \mu \mathrm{s})$ was applied with a $3.6 \mathrm{~s}$ delay between experiments. Velocity was probed by stepping the PGSTE gradient ( $q$-gradient) linearly for eight values ranging from 0 to $0.3625 \mathrm{~T} \mathrm{~m}^{-1}$. The duration of the $q$-gradient, $\delta$, and the time delay between $q$-gradients, $\Delta$, ranged from 1 to $2 \mathrm{~ms}$ and from 10 to $75 \mathrm{~ms}$, respectively. 
Two slice selective pulses were applied to restrict the imaged sample dimensions to $2 \mathrm{~mm}$ in the flow direction and $20 \mathrm{~mm}$ along the vorticity axis. A $500 \mathrm{kHz}$ acquisition bandwidth was used to reduce water-oil H-1 chemical shift artefacts and 512 complex points were collected, with a $20 \mathrm{~mm}$ field of view. The nominal voxel size was therefore $2 \mathrm{~mm} \times 40 \mu \mathrm{m} \times 20 \mathrm{~mm}$ (flow direction $\times$ across gap $\times$ depth). A four-step cyclically ordered phase cycle (CYCLOPS $)^{23}$ was employed for each $q$-step, resulting in a total time for each velocity image of about 2 min. The $q$-dimension was zero-filled to 512 and a one-dimensional velocity map was obtained via Fourier transformation with respect to the $q$-dimension for each voxel.

\subsubsection{Rheology}

Rheology is the study of the flow and deformation of matter. The experimental characterisation of a material's rheological behaviour is known as rheometry, although the term rheology is frequently used synonymously with rheometry, particularly by experimentalists. One generally associates liquids with viscous behaviour and solids with elastic behaviour. A more precise way in defining solids and liquids is to consider the material behaviour at short times (relative to the duration of the experiment/application of interest) and at long times. Such a definition may use one of the dimensionless numbers in rheology, the Deborah number:

$$
D_{e}=\frac{t_{c}}{t_{p}}
$$

where $t_{c}$ is the time characterising the intrinsic fluidity of the material and $t_{p}$ is the time scale of observation. When $D_{e} \leq 1$, the material is less resistant to flow and appears as liquid. Large Deborah numbers $\left(D_{e} » 1\right)$ correspond to situations where the material behaves rather elastically. The Deborah number is relevant for materials that flow on long time scales but not for the materials that are viscous on short time scales but solid on the long term.

\subsubsection{Elastic and Viscous, Newtonian and non-Newtonian}

From a different perspective, in this section we will discuss the views of Robert Hooke and Isaac Newton on the concepts of elastic and viscous behaviours. According to Hooke's Law $F=k x$ where $F$ is the restoring force exerted by the material (spring), $x$ is the distance that the 
spring has been stretched or compressed and $k$ is the force constant (or spring constant), the stress of $\sigma$ that is applied to any material yielding an instantaneous deformation called the strain (strain is defined as the deformation relative to a reference ${ }^{10}$ ) can be written as

$$
\sigma=\gamma G
$$

where $G$ is the rigidity modulus (or shear modulus) of the material. In other words, when a constant stress is applied to a material, it deforms immediately by an amount $\gamma$ and returns to the equilibrium position which is the position where the material would naturally come to rest, the material is referred to as being elastic.

Isaac Newton introduced the idea that stress can be written as

$$
\sigma=\eta \dot{\gamma}
$$

where $\dot{\gamma}$ is the velocity of a flowing material and $\eta$ is a degree of slip retardancy of the material and is called the viscosity. When a constant stress is applied to a Newtonian fluid, it deforms immediately and continues to flow at $\dot{\gamma}$ until the applied stress ceases. The deformation achieved over the applied stress is not recovered and the material is referred to as being viscous. In Newtonian fluids, the Brownian motion and interparticle forces are completely balanced and correspond to constancy in the viscosity.

Equation 2.27 is no longer applied to non-Newtonian fluid because the viscosities of the material are now a function of the applied shear rate $(\dot{\gamma})$. Under continuous steady shear, emulsions exhibit either Newtonian or non-Newtonian fluids, i.e., shear-thinning and shearthickening fluids. Average droplet size, droplet size distribution, volume fraction ${ }^{24}$, oil-water interfacial properties and the forces between the droplets ${ }^{25}$ define the rheological behaviours of emulsions. For shear-thinning fluids, an increase in shear rate disrupts the packing of droplets. The droplets are deformed, elongated and aligned in the direction of the shearing and eventually leading to a decrease in viscosity ${ }^{26,27}$. Shear-thickening fluids can be identified where there is an increase in viscosity with a possible development of highly associated microstructure ${ }^{28}$. Fig. 2.14 shows schematic flow curves according to several models ${ }^{29}$ that have been developed to describe the flow behaviours of fluids.

In the following section, we will discuss two of the most common geometries that we used as rotational devices for the study of the shear flow behaviour of our samples. 


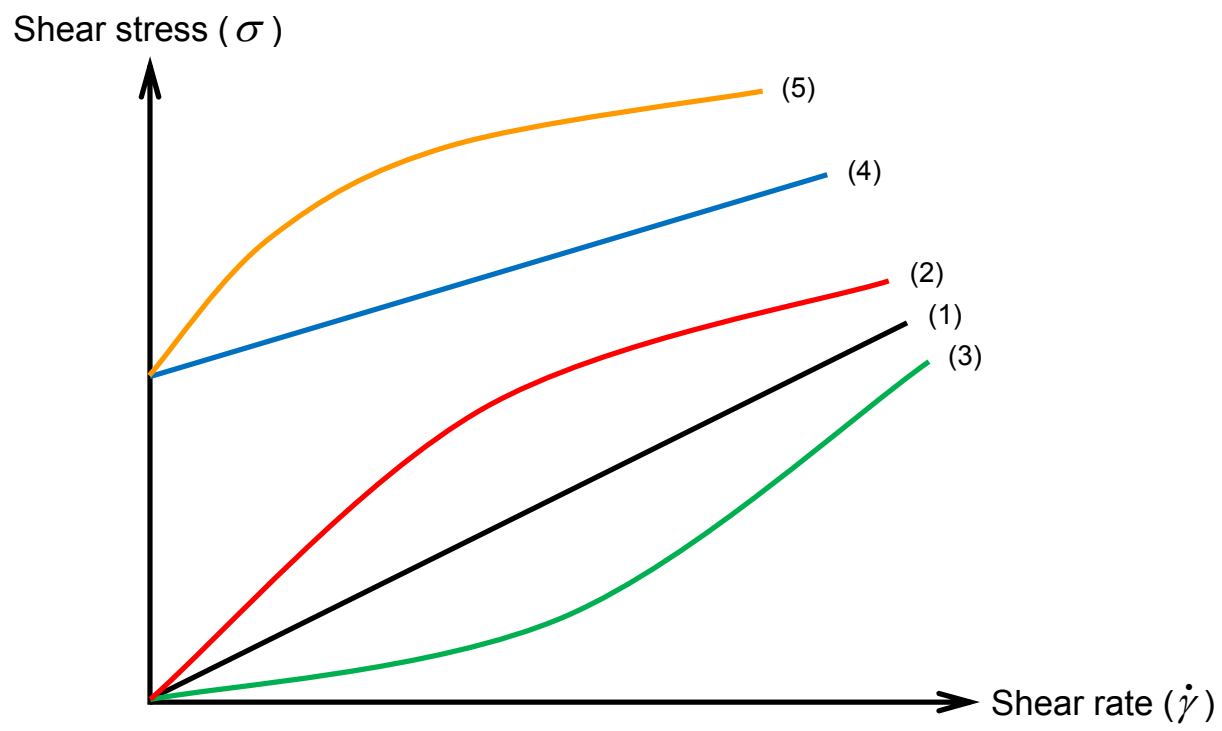

Figure 2.14: Shear stress vs. shear rate flow curves for (1) Newtonian liquid, (2) shear-thinning fluid, (3) shearthickening fluid and according to (4) Bingham model where the materials begins to flow after a certain stress has been applied resulting in a linear stress with a non-zero intercept and (5) Herschel-Bulkley model which is similar to the Bingham model except the stress is not linear.

\subsubsection{Geometries}

\subsection{Cone-and-Plate}

A schematic of the cone-and-plate geometry is shown in Fig. 2.15. When the cone of angle $\Theta_{0}$ (normally a few degrees) is rotated at a constant angular velocity $\Omega$, shear flow in the $\phi$ direction is created. The distance between the cone and the plate is given by $d=r \sin \Theta_{0}$ where $r$ is the measured distance from the cone apex (Fig. 2.15). Hence, in the limit of small cone angles, material in any part of the gap will have the same velocity $U=\Omega r$ and since $\sin \Theta_{0} \approx 1$, the shear rate, $U / d$ can be written as

$$
\dot{\gamma}=\frac{\Omega}{\Theta_{0}} .
$$

Because the shear rate is constant for the cone-and-plate geometry, the shear stress throughout the flow domain is also constant;

$$
\sigma=\frac{3 \Gamma}{2 \pi R^{3}}
$$


where $\Gamma$ is the torque required to obtain a constant motion and $R$ is the radius of the cone. Thus the viscosity (of a Newtonian fluid) in cone-and-plate flow may be calculated directly:

$$
\eta=\frac{3 \Gamma \Theta_{0}}{2 \pi R^{3} \Omega} .
$$

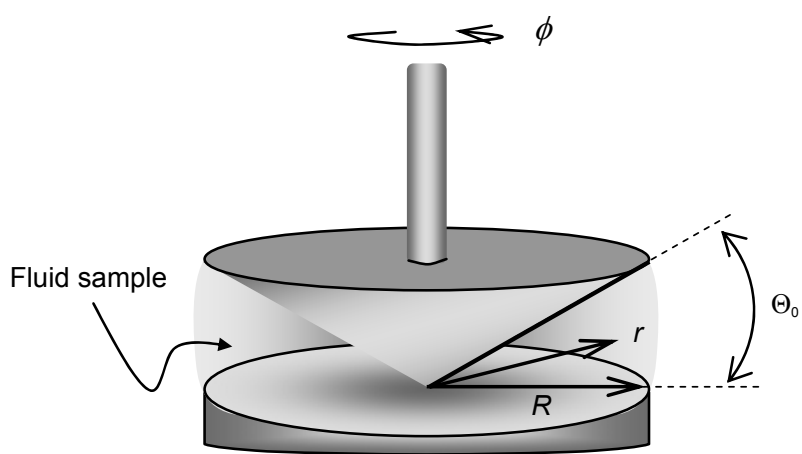

Figure 2.15: Schematic diagram for a cone-and-plate geometry: a cone of angle $\Theta_{0}$ with a radius $R$ is rotated at an angular velocity $\Omega$ in $\phi$-direction. The sample is placed between the cone and the plate ${ }^{11}$.

\subsection{Concentric-cylinder Couette}

A schematic of the cross section of the Couette geometry is shown in Fig. 2.16. When the inner cylinder is rotated at a constant angular velocity or relative velocity between the two cylinders, $\Omega_{0}$, simple shear flow takes place in the $\phi$-direction. The boundary conditions are the velocity, $v=0$ at $r=R$ and $v=\kappa R$ at $r=\kappa R$ where $R$ and $\kappa R$ are the radii of the outer and the inner cylinders, respectively. The gap-averaged shear rate $\dot{\gamma}$ is

$$
\dot{\gamma}=\frac{\kappa \Omega_{0}}{1-\kappa} \text {. }
$$

The shear stress can be obtained through the following manipulations:

$$
\begin{gathered}
\Gamma=(\text { stress })(\text { lever arm })(\text { area }) \\
=(\sigma)(\kappa R)(2 \pi \kappa R L) \\
\sigma=\frac{\Gamma}{2 \pi R^{2} \kappa^{2} L}
\end{gathered}
$$

where $L$ is the length of the inner cylinder. Using equations 2.31 and 2.33 , the viscosity of a Newtonian fluid can be determined:

$$
\eta=\frac{\Gamma(1-\kappa)}{2 \pi R^{2} \kappa^{3} L \Omega_{0}} .
$$




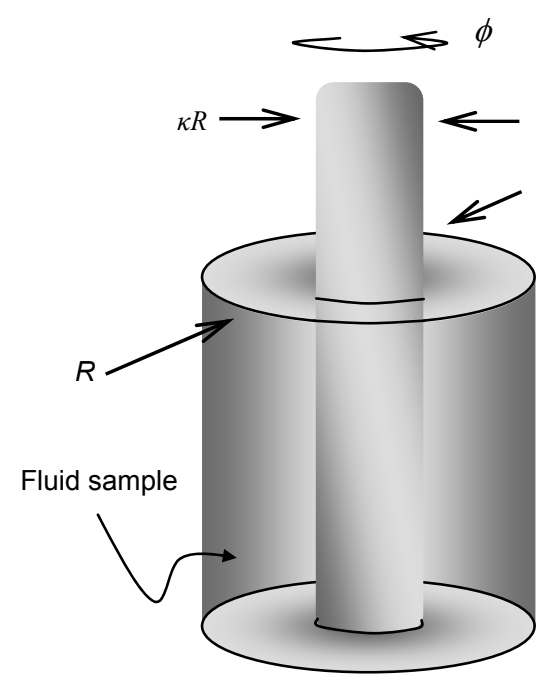

Figure 2.16: Schematic diagram for a Couette geometry: the inner cylinder is rotated at a constant angular velocity $\Omega_{0}$ in the $\phi$-direction. The sample is placed between the two cylinders ${ }^{11}$.

\subsubsection{Experimental Parameters}

The transient response of Na-caseinate dispersions to continuous steady shear at various rates for up to $60000 \mathrm{~s}(\sim 17 \mathrm{hrs})$ was investigated using a strain-controlled ARES rheometer in conjunction with a quartz cone-and-plate geometry (diameter $50 \mathrm{~mm}$, angle $0.04 \mathrm{rad}$ and truncation $48 \mu \mathrm{m})$.

For emulsions, non-linear rheology measurements, unless stated otherwise, were performed using a stress-controlled TA Instruments AR2000 rheometer in conjunction with a steel cone-and-plate (diameter $60 \mathrm{~mm}$, truncation angle $2^{\circ}$ ). Peak hold experiments were performed to study the stress response of the emulsions as a function of time. The measurements were carried out by varying the shear rate logarithmically from 0.0001 to 1000 $\mathrm{s}^{-1}$ with 10 steps per decade and also in reverse. The sample was studied by applying a shear rate for a fixed time period (from 1 to 15 minutes). The sample was then left unsheared for a waiting time of $1 \mathrm{~min}$ before the next shear rate was applied. Data were collected and averaged over the final $10 \mathrm{~s}$ to obtain a flow curve. This protocol was followed for both the up-ramping and down-ramping sweeps.

Specifically, the stress response of the materials was also investigated by subjecting the samples to a range of constant shear rates from 0.01 to $0.1 \mathrm{~s}^{-1}$ over $6000 \mathrm{~s}$. Creep experiments were performed to study the strain response of the emulsions as a function of time. The experiments were carried out by applying torques ranging from 0.1 (the limit of 
the instrument) to $0.8 \mu \mathrm{N}$ m over $1500 \mathrm{~s}$. For both types of experiment above, fast sampling mode was used, with the data being recorded one point every $1 \mathrm{~s}$. For both rheometers, the temperature was controlled at $25^{\circ} \mathrm{C}$ by a Peltier system. A solvent trap was used to minimise drying of the sample during the measurements.

\subsubsection{Linear Rheology}

The linear viscoelastic response of a material is determined by applying an oscillatory strain and simultaneously recording the corresponding stress response. Thus, the strain and shear rate are

$$
\begin{gathered}
\gamma(t)=\gamma_{0} \sin (\omega t), \dot{\gamma}=\gamma_{0} \omega \cos (\omega t) \text { and } \\
\sigma(t)=\gamma_{0}\left[G^{\prime} \sin (\omega t)+G^{\prime \prime} \cos (\omega t)\right]
\end{gathered}
$$

where $\gamma_{0}$ is the amplitude of oscillation, $\omega$ is the applied angular frequency and $G^{\prime}$ and $G^{\prime \prime}$ are the storage and loss moduli, respectively. The complex modulus, $G^{*}(\omega)$ is given by:

$$
G^{*}(\omega)=G^{\prime}(\omega)+i G^{\prime \prime}(\omega) .
$$

Typically, the time dependent stress $\tau_{0}(\omega)$ of the material is studied by applying a perturbative sinusoidal strain. $\gamma_{0}(\omega)$ is expected to be shifted by a phase angle $\delta$ from the sinusoidal strain. The elastic (storage, $G^{\prime}$ ) and viscous (loss, $G^{\prime \prime}$ ) moduli are then calculated as:

$$
\begin{gathered}
G^{\prime}(\omega)=\frac{\tau_{0}(\omega)}{\gamma_{0}} \cos \delta(\omega) \text { and } \\
G^{\prime \prime}(\omega)=\frac{\tau_{0}(\omega)}{\gamma_{0}} \sin \delta(\omega)
\end{gathered}
$$

where

$$
\tan \delta=G^{\prime \prime} / G^{\prime} .
$$

This relationship is depicted in Fig. 2.17 where the normalised strain (input)/stress (output) is shown for phase angles $\delta=0^{\circ}, 45^{\circ}$ and $90^{\circ}$ corresponding to a purely elastic response, an equal mixture of elastic and viscous responses and a purely viscous response, respectively. 


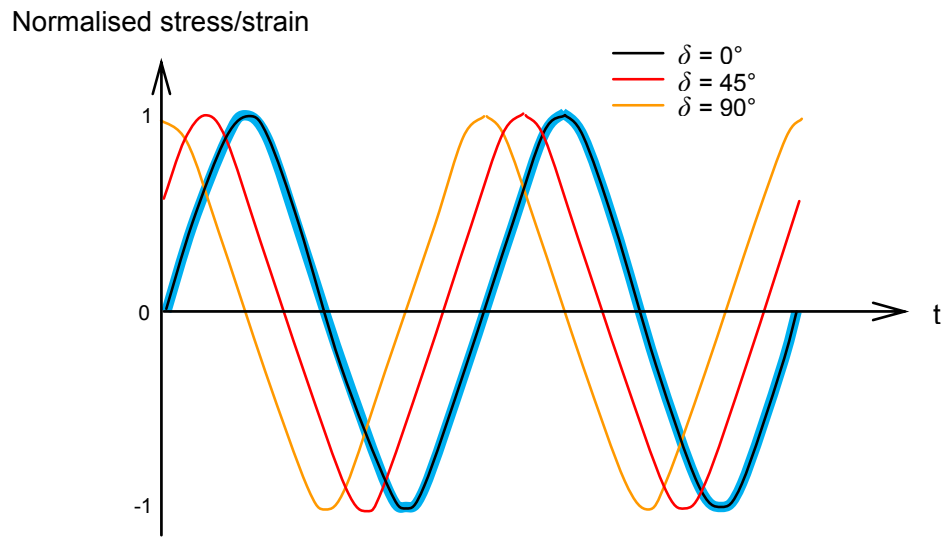

Figure 2.17: Schematic of the stress response as a function of time for a Newtonian fluid at phase angles of $0^{\circ}$, $45^{\circ}$ and $90^{\circ}$ with the strain input being marked as the blue line.

The Cross model, named after Malcolm Cross $^{30}$ is an empirical model and is amongst the simplest models used to describe the dynamic response of a non-Newtonian fluid to an oscillatory strain. The viscosity of the fluid for all applied shear rates/shear stresses is no longer constant but rather is a function of the shear rate and follows a power law model:

$$
\eta(\dot{\gamma})=K \dot{\gamma}^{n-1} .
$$

$K$ ( $\mathrm{Pa} \mathrm{s})$ is the coefficient of proportionality and $n$ (dimensionless) is the power law index. When $n<1, n=1$ and $n>1$ as illustrated in Fig. 2.18, the material is empirically classified as strain-softening, Newtonian and strain-thickening, respectively.

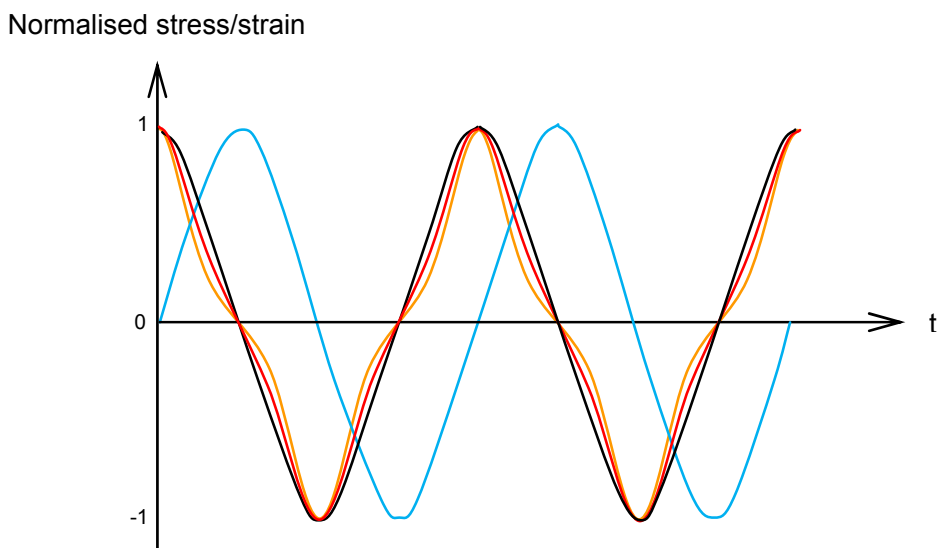

Figure 2.18: Schematic of the stress response as a function of time for a non-Newtonian fluid at $n=0.5$ (strainsoftening), 1.0 (Newtonian) and 1.5 (strain-thickening) (dark to light) with the strain input being marked as the blue line. 


\subsection{Experimental Parameters}

For Na-caseinate dispersions, dynamic strain sweep and frequency sweep measurements were performed at a frequency of $1 \mathrm{~Hz}$ for strain amplitudes of 0.1 to $1000 \%$ and from 0.01 to 50 $\mathrm{Hz}$ at a strain amplitude of $1 \%$, respectively using a strain-controlled ARES rheometer in conjunction with a quartz cone-and-plate geometry (diameter $50 \mathrm{~mm}$, angle $0.04 \mathrm{rad}$ and truncation $48 \mu \mathrm{m})$. Strain sweep measurements were also carried out at a frequency of $1 \mathrm{~Hz}$ for strain amplitudes of 0.1 to $10000 \%$ using a stress-controlled TA Instruments AR2000 rheometer in conjunction with an acrylic cone-and-plate geometry (diameter $30 \mathrm{~mm}$, truncation angle $2^{\circ}$ ).

For emulsions, dynamic strain sweep and frequency sweep measurements were performed at a frequency of $1 \mathrm{~Hz}$ for strain amplitudes of 0.01 to $100000 \%$ and from 0.01 to $100 \mathrm{~Hz}$ at a strain amplitude of $2 \%$, respectively using a stress-controlled TA Instruments AR2000 rheometer in conjunction with an acrylic cone-and-plate geometry (diameter $30 \mathrm{~mm}$, truncation angle $1^{\circ}$ ). For both rheometers, temperature was controlled at $25^{\circ} \mathrm{C}$ by a Peltier system. A solvent trap was used to minimise drying of the sample during the measurements.

\subsubsection{Cryo-Scanning Electron Microscopy (Cryo-SEM)}

Scanning electron microscopy (SEM) is an imaging technique that employs an illuminating electron beam in an operating vacuum. It creates images by probing the sample with a focused electron beam that passes through pairs of scanning coils, deflecting the beam in the $x$ and $y$ axes so that it scans in a raster fashion over a rectangular area of the specimen. When the primary electrons interact with the atoms at or near the surface of the sample, the electrons lose energy by repeated random scattering and absorption within an interaction volume (depending on the electron's landing energy, the atomic number of the specimen and the specimen's density) of the specimen, which extends from less than $100 \mathrm{~nm}$ to around 5 $\mu \mathrm{m}$ into the surface.

The energy exchange between the electron beam and the sample results in the emission

of secondary electrons, back-scattered electrons, characteristic x-rays, light (cathodoluminescence), specimen current and transmitted electrons (i.e., the signals), each of which can be detected by specialised detectors. The signals are amplified and displayed as variations in brightness on a cathode ray tube (CRT). The SEM image is therefore a 
distribution map of the intensity of the signal being emitted from the scanned area of the specimen. The image is digitally captured and displayed on a screen. Magnification is controlled by the current supplied to the $x$ and $y$ scanning coils. Magnetic lenses magnify the image from the ratio of the dimensions of the raster on the specimen to the raster on the display screen. Assuming that the display screen has a fixed size, higher magnification can be achieved by reducing the size of the raster on the specimen, and vice versa.

Secondary electron imaging (SEI), the most common or conventional imaging mode is used in acquiring images of our emulsion samples. This imaging method collects low-energy $(<50 \mathrm{eV})$ secondary electrons that are ejected from the k-orbitals of the specimen atoms by inelastic scattering interactions with the electron beam. The number of secondary electrons reaching the detector determines the brightness of the signal. In scanning electron microscopy, the emission of secondary electrons is limited to the area adjacent to the point of incidence. If the beam enters the sample perpendicular to the surface, then the activated region is uniform about the axis of the beam and the quantity of secondary electrons that "escape" from the specimen surface is minimal. As the angle of incidence increases, the "escape" distance of one side of the beam will decrease, and therefore more secondary electrons will be emitted. Thus steep surfaces and edges tend to be brighter than flat surfaces, which results in images with a well-defined, three-dimensional appearance. This process is illustrated in Fig. 2.19.

(a)

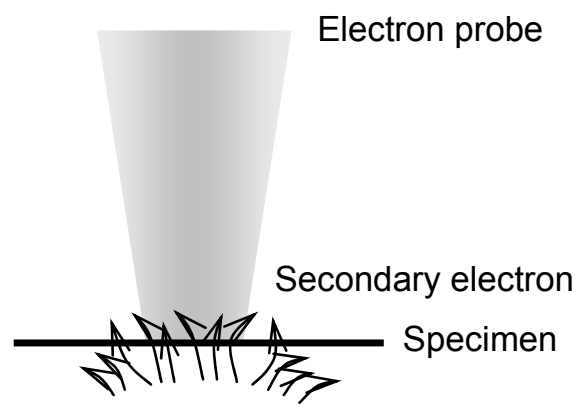

(b)

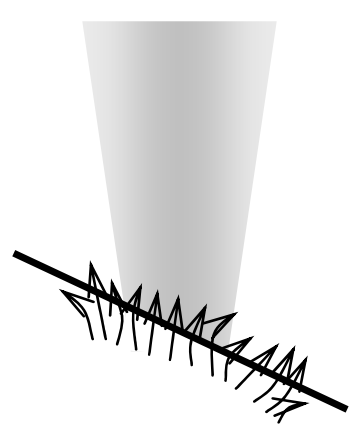

(c)

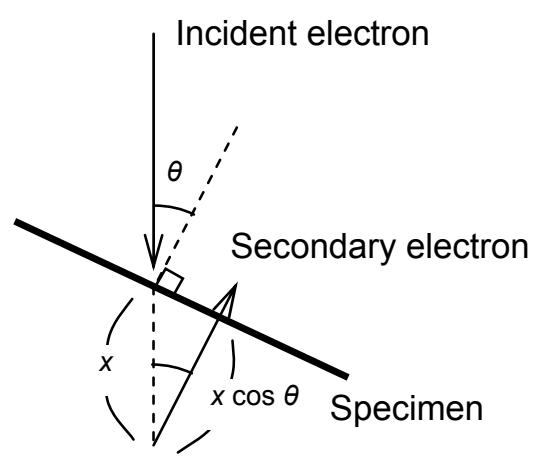

Figure 2.19: Secondary electron emission at a tilt angle perpendicular to the specimen surface (a): the amount of secondary electrons that "escape" from the specimen surface is relatively small resulting in a darker image; and tilt angle $\theta(\mathrm{b})$ : more secondary electrons "escape" from one side of the specimen surface resulting in a brighter image. In (c) is shown the secondary electrons produced at a depth $x$ that will travel along the shortest distance $x \cos \theta$ to the specimen surface ${ }^{14}$. 
Cryo-SEM is of great value in studying samples which would otherwise be impossible to image in the SEM, such as samples which are liquid at room temperature. The preparation process involves rapidly freezing the sample at cryogenic temperatures (generally in liquid nitrogen slush) to produce a vitreous or non-crystalline specimen, preserving the sample in a near-native state without the distortions from stains or fixatives needed for traditional EM. When the frozen specimen is fractured under cryogenic conditions, the material breaks clean along weak edges without causing much malformation but allows optimum exposure of internal microstructure, topographical arrangement and three-dimensional surface morphology in a system such as an emulsion or suspension. High resolution imaging is carried out after a thin conductive coating is applied to the freeze-fracture specimen.

\subsubsection{Resolution and Depth of Field}

The quality of images is largely determined by the resolving power of the microscope. The resolving power is defined as the shortest distance between two points which can be recognised as two different images. However, the electrical and mechanical stability of the electron-optical system, the signal-to-noise ratios of the detector and amplifier, the performance of the scanning circuits and recording system (or CRT) are the factors that may restrict the resolving power of the scanning electron microscope. In addition, specific specimen preparation, the instrument operating conditions and photographic processing must be taken into consideration to ensure optimal resolution for the specimen of interest.

Assuming $d$ is the resolving power of the microscope. If we are to identify the resolution of an image, the image must be enlarged to the magnification whose minimum value, i.e., the effective magnification $M$ is

$$
M=\frac{d_{1}}{d}
$$

where $d_{1}$ is the resolution of the human eye (approximately $0.1 \mathrm{~mm}$ ). With a scanning electron microscope the depth of field is very large providing a clear observation of the characteristic three-dimensional appearance and protruding spikes which are useful for allowing the surface structure of a sample to be determined. 


\subsubsection{Experimental Parameters}

A Jeol JSM_6500F fitted with a Gatan Alto 2500 cryo attachment, operated at $5 \mathrm{kV}$ and $3 \times$ $10^{-11}$ A was used to perform cryo-scanning electron microscopy (cryo-SEM) allowing the internal structure of the emulsions to be visualised. Freezing is generally achieved within $1 \mathrm{~s}$. The sample was mounted on the sample holder and plunged frozen into "slushy" nitrogen (a mixture of solid and liquid nitrogen at a constant temperature of $-190^{\circ} \mathrm{C}$ having a greater refrigerant capacity than straight liquid nitrogen). The sample holder was then withdrawn, under vacuum, into a vacuum transfer device for transfer to the cryo-preparation chamber. After transfer to the (separately pumped) cryo-preparation chamber the sample was maintained at a low temperature of $-130^{\circ} \mathrm{C}$ and low contamination conditions. The sample was fractured using a cold knife to expose the internal structure and left for sublimation at $110^{\circ} \mathrm{C}$ for approximately 30 minutes. Finally, a thin conductive coating of platinum was applied to allow high resolution imaging in the SEM. Transfer to the SEM chamber was via an interlocked airlock and onto a cold stage module fitted to the SEM stage maintained at a temperature of $-130^{\circ} \mathrm{C}$. 


\subsection{References}

1. I. Burgaud, E. Dickinson and P. V. Nelson, Int. J. Food Sci. \& Tech. 25 (1), 39-46 (1990).

2. $\quad$ T. Allen, Particle size measurement, 4th ed. (Chapman \& Hall 1992).

3. C. F. Bohren and D. R. Huffmann, Absorption and scattering of light by small particles. (Wiley-Interscience, New York, 1983).

4. M. I. Mishchenko, J. W. Hovenier and L. D. Travis, Light scattering by nonspherical particles: theory, measurements, and applications. (Academic Press, San Diego, 2000).

5. J. P. M. Syvitski, Principles, methods and application of particle size analysis. (Cambridge University Press, 2007).

6. A. Abragam, Principles of nuclear magnetism. (Clarendon Press, Oxford, 1961).

7. C. Slichter, Principles of magnetic resonance, 3rd ed. (Springer, Berlin, 1990).

8. P. T. Callaghan, Principles of nuclear magnetic resonance microscopy, 1 st ed. (Oxford Science Publishing, Oxford, 1991).

9. E. Fukushima and S. Roeder, Experimental pulse NMR - a nuts and bolts approach. (Addison-Wesley Publishing Company, Inc., 1981).

10. J. F. H. H. A. Barnes and K. Walters, An introduction to rheology, 1st ed. (Elsevier, Amsterdam, 1989).

11. F. A. Morrison, Understanding rheology. (Oxford University Press, New York, 2001).

12. R. G. Larson, The structure and rheology of complex fluids, 1st ed. (Oxford University Press, New York, 1999).

13. T. E. Faber, Fluid dynamics for physicists, 1st ed. (Cambridge University Press, UK, 1995).

14. E. Dickinson and J. Chen, J. Agric. Food Chem. 46, 91-97 (1998).

15. G. I. Goldstein, D. E. Newbury, P. Echlin, D. C. Joy, C. Fiori and E. Lifshin, Scanning electron microscopy and $x$-ray microanalysis. (Plenum Press, New York, 1981).

16. H. Y. Carr and E. M. Purcell, Phys. Rev. 94, 630-638 (1954).

17. S. Meiboom and D. Gill, Rev. Sci. Instr. 29, 688-691 (1958). 
18. E. O. Stejskal and J. E. Tanner, J. Chem. Phys. 42, 288-292 (1965).

19. D. E. Woessner, J. Chem. Phys. 34, 2057-2061 (1961).

20. I. Lönnqvist, A. Khan and O. Söderman, J. Colloid Interface Sci. 144, 401-411 (1991).

21. C. L. Lawson and R. J. Hansen, Solving least squares problems, 1st ed. (Prentice Hall, Englewood Cliffs, NJ, 1974).

22. A. Raudsepp, K. W. Feindel and Y. Hemar, Rheol. Acta 49, 371-379 (2010).

23. D. I. Hoult and R. E. Richards, Proc. Roy. Soc. A344, 311-340 (1975).

24. J. Weiss, C. Canceliere and D. J. McClements, Langmuir 16, 6833-6838 (2000).

25. R. Buscall, J. Chem. Soc., Faraday Trans. 87, 1365-1370 (1991).

26. A. C. Rust and M. Manga, J. Non-Newtonian Fluid Mechanics 104, 53-63 (2002).

27. D. J. Stein and F. J. Spera, J. Volcanology Geothermal Res. 49, 157-174 (1992).

28. M. Ballard, R. Buscall and F. Waite, Polymer 29, 1287-1293 (1988).

29. R. W. Whorlow, Rheological techniques. (Chichester: E. Horwood, Halsted Press, New York, 1980).

30. M. M. Cross, J. Colloid Interface Sci. 20, 417-437 (1965). 


\section{Chapter 3}

\section{$\mathcal{N a - c a s e i n a t e ~ D i s p e r s i o n s}$}

\subsection{Introduction}

Na-caseinate aggregates in solution to form the so-called "submicelles" of molecular weight $\approx 3 \times 10^{5} \mathrm{Da}$ (12 to 15 protein monomers) and radius 5 to $10 \mathrm{~nm}^{1-7}$. These submicelles coexist in equilibrium with free casein molecules $\left(\approx 3 \times 10^{4} \mathrm{Da}\right)^{8}$. Electrostatic forces combined with hydrogen bonding and van der Waals attractions account for the association of casein. The degree of aggregation varies due to the irregular and heterogeneous distribution of particle charges. Under Brownian motion and disruption forces like mechanical agitation the system is constantly reshaping. As the molecular structures of caseins are dictated by the local environment, they are known as 'rheomorphic' proteins; that is, they are flexible enough to 'go with flow'. Thus, it is obvious that the interactions between casein or casein aggregates in aqueous solution may have significant influence over either or both the formulation characteristics and the final qualities of casein-based products.

However, details of the structures adopted by casein in native buffer solution (the continuous phase in our emulsion system), the relationship between the extent of aggregation, gelation and ageing of the casein aggregates and their correspondence to native casein micelles has not yet been fully elucidated. A recent investigation by Mariette et al. showed that the water diffusion coefficients of native micellar casein dispersions and those of aggregates formed by commercial Na-caseinate in buffer solution were identical due to the similarity of the aggregate microstructure and that this evolved on increasing protein concentration $^{10}$. This indicates that Na-caseinate in buffer solution is a good model system for investigations focused on gaining understanding of the underlying physics of such complex self-assembling systems and in particular food systems.

Rheological and ageing studies on such systems are scant, yet information yielded from these types of investigations is crucial to the use and future application of casein-based 
systems. As such, to more fully probe the self-assembly of Na-caseinate we present here a study on the interactions of casein aggregates in buffer solution using three complementary experimental techniques: laser diffraction particle sizing, diffusion NMR (PGSTE-NMR) and rheology. Together these offer us a means of determining the microstructural and rheological properties of the resulting soft matter probed over a large range of Na-caseinate concentrations.

\subsection{Results}

Direct observation of the samples gave evidence of an apparent transition based on both sample turbidity and viscosity upon increasing Na-caseinate concentration. This can be viewed in Fig. 3.1. At the lowest concentrations $<6 \mathrm{wt} \%$ Na-caseinate the viscosity of the solutions is comparable to that of water and the turbidity of the samples is very weak. Turbidity is markedly increased from $6 \mathrm{wt} \%$ onwards, with the solutions taking on an opalescent quality on increasing concentration. Viscosity increases significantly from $\sim 10$ $\mathrm{wt} \%$. Samples with $16 \mathrm{wt} \% \mathrm{Na}$-caseinate and higher are solid-like, they do not flow when tipped upside down.

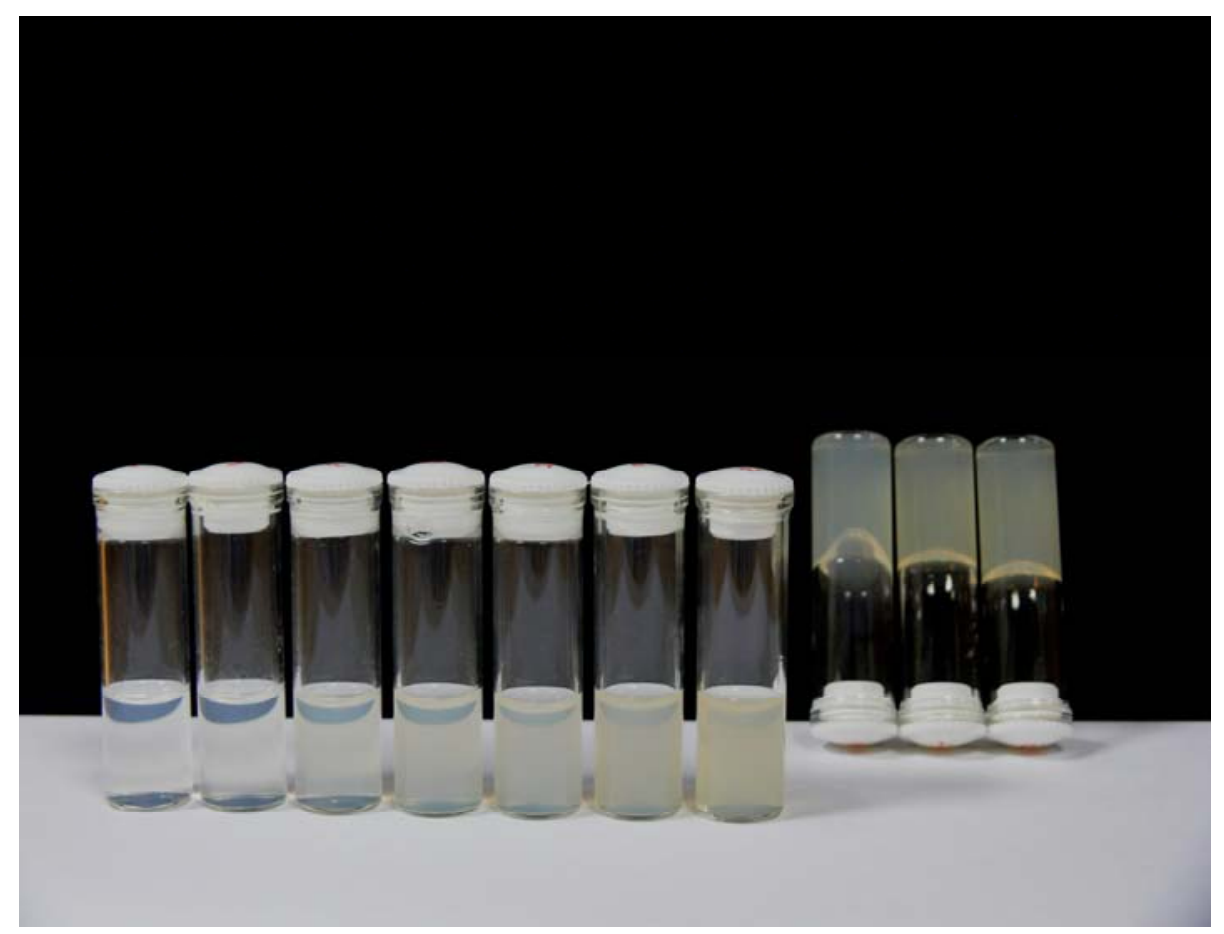

Figure 3.1: Photograph of 2 to $20 \mathrm{wt} \% \mathrm{Na}$-caseinate dispersions in $2 \mathrm{wt} \%$ increments, from left to right. 


\subsubsection{Laser Diffraction Particle Sizing}

In Fig. 3.2 is shown a representative aggregate size distribution of the Na-caseinate dispersions $(10 \mathrm{wt} \%)$. The distribution is largely unchanged on varying Na-caseinate concentration (see Appendix; the high end peak becomes slightly more pronounced on increasing Na-caseinate concentration, this has a significant influence with regard to the PGSTE-NMR data) and aggregates from $\sim 0.04$ to $0.84 \mu \mathrm{m}$ in diameter exist. The mean aggregate diameter is $0.133 \mu \mathrm{m}$ which is similar to the average size of native casein micelles in milk $(0.1 \text { to } 0.2 \mu \mathrm{m})^{11}$.

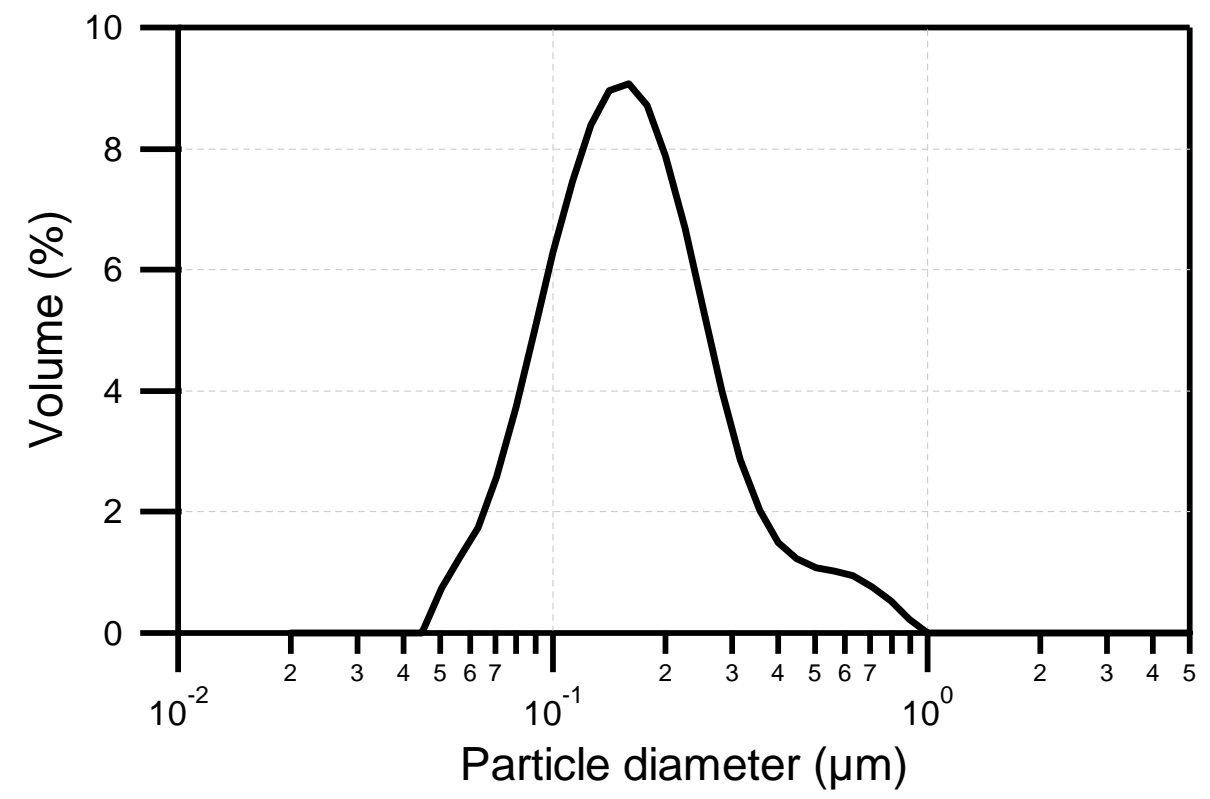

Figure 3.2: A representative aggregate size distribution for Na-caseinate dispersions, indicating aggregate formation across the size range of $\sim 40 \mathrm{~nm}$ to $\sim 1 \mu \mathrm{m}$, here for a $10 \mathrm{wt} \%$ sample.

\subsubsection{PGSTE-NMR}

The water diffusion coefficients of Na-caseinate dispersions as a function of Na-caseinate concentration are shown in Fig. 3.3. These values are in agreement with the work of Mariette et al. ${ }^{10}$. The water diffusion coefficient decreases as Na-caseinate concentration increases. At low Na-caseinate concentrations, the diffusion coefficient of water $D_{\text {eff }}$ is similar to that for bulk water $\left(D_{\mathrm{o}}=2.3 \times 10^{-9} \mathrm{~m}^{2} \mathrm{~s}^{-1}\right)$. As we decrease the amount of water in the system, the ratio of $D_{\text {eff }} / D_{\mathrm{o}}$ gradually decreases. The diffusion coefficient of water in all samples 
remains, however, on the same order of magnitude as that of bulk water. For any given sample no variation in $D_{\text {eff }}$ was observed for $\Delta$ between 20 and $200 \mathrm{~ms}$ and the echo attenuation data for water showed a single exponential decay for all concentrations. Hence the majority of the water molecules undergo unrestricted diffusion at all concentrations over a length scale, given by the relationship

$$
\left\langle Z^{2}\right\rangle^{1 / 2}=\sqrt{2 D \Delta}
$$

Hence, water molecules diffuse $\sim 9.3 \mu \mathrm{m}$ for a Na-caseinate concentration of $0.02 \mathrm{~g} / \mathrm{g}$ ( $\mathrm{g}_{\text {casein }} / \mathrm{g}_{\text {water }}$ ) and $\sim 7.4 \mu \mathrm{m}$ for a concentration of $0.25 \mathrm{~g} / \mathrm{g}$.

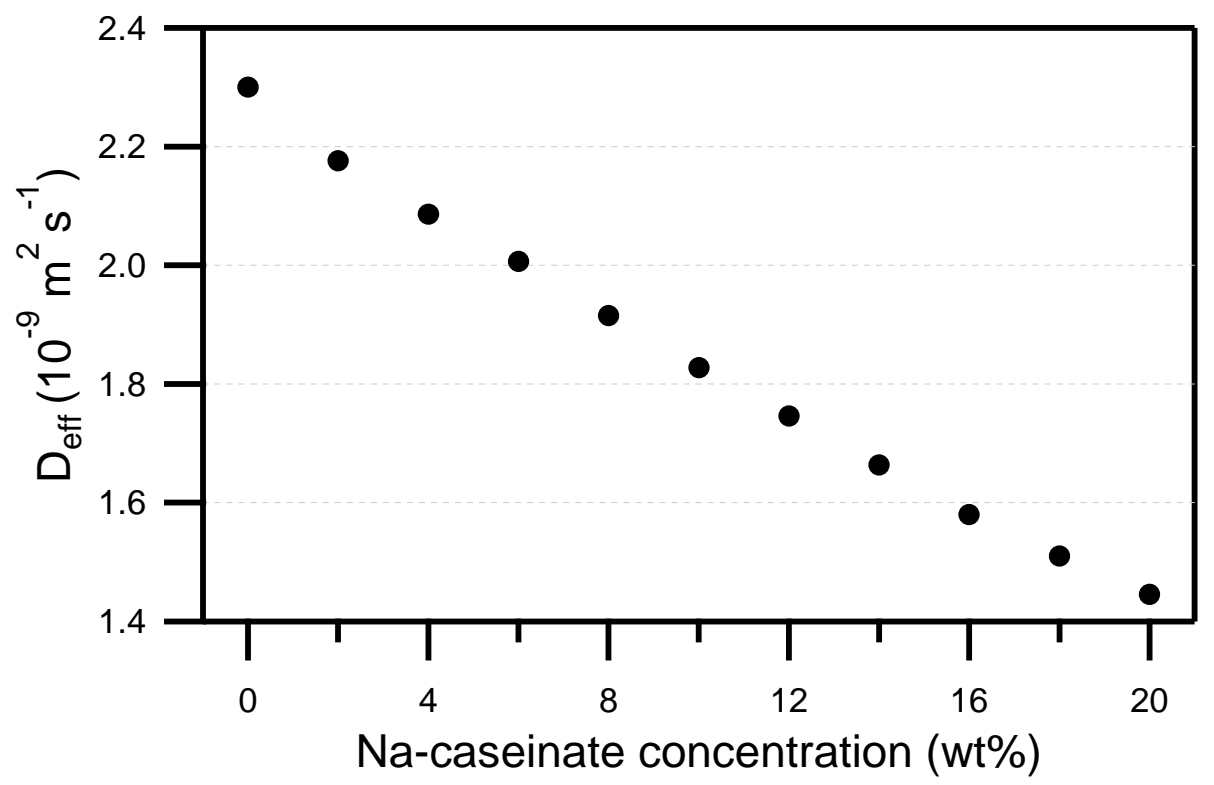

Figure 3.3: Observed water diffusion coefficients as a function of Na-caseinate concentration, $\Delta=20 \mathrm{~ms}$.

In the context of acquiring echo attenuation for casein aggregates, a strong gradient pulse was applied to suppress the water signal. Due to the presence of a small amount of free protein in the Na-caseinate powder, their contributions to the proton signal of casein aggregates cannot be neglected ${ }^{10}$. Consequently, the attenuation curve of the NMR echo signal for all casein aggregate diffusion coefficient measurements showed a non-linear decay. In Fig. 3.4 is shown a characteristic set of attenuation data, here for a $6 \mathrm{wt} \%$ Na-caseinate dispersion. The casein aggregate diffusion coefficient was determined by performing a linear fit to the attenuation data in the non-shaded zone. This diffusion coefficient is extracted on an ensemble average basis. Inverse Laplace transformations were also performed on the attenuation data, with the peak in the so-obtained diffusion coefficient distribution 
corresponding to the value obtained from the linear fitting procedure. This will be addressed in more detail in the discussion but for what follows the ensemble average value is used.

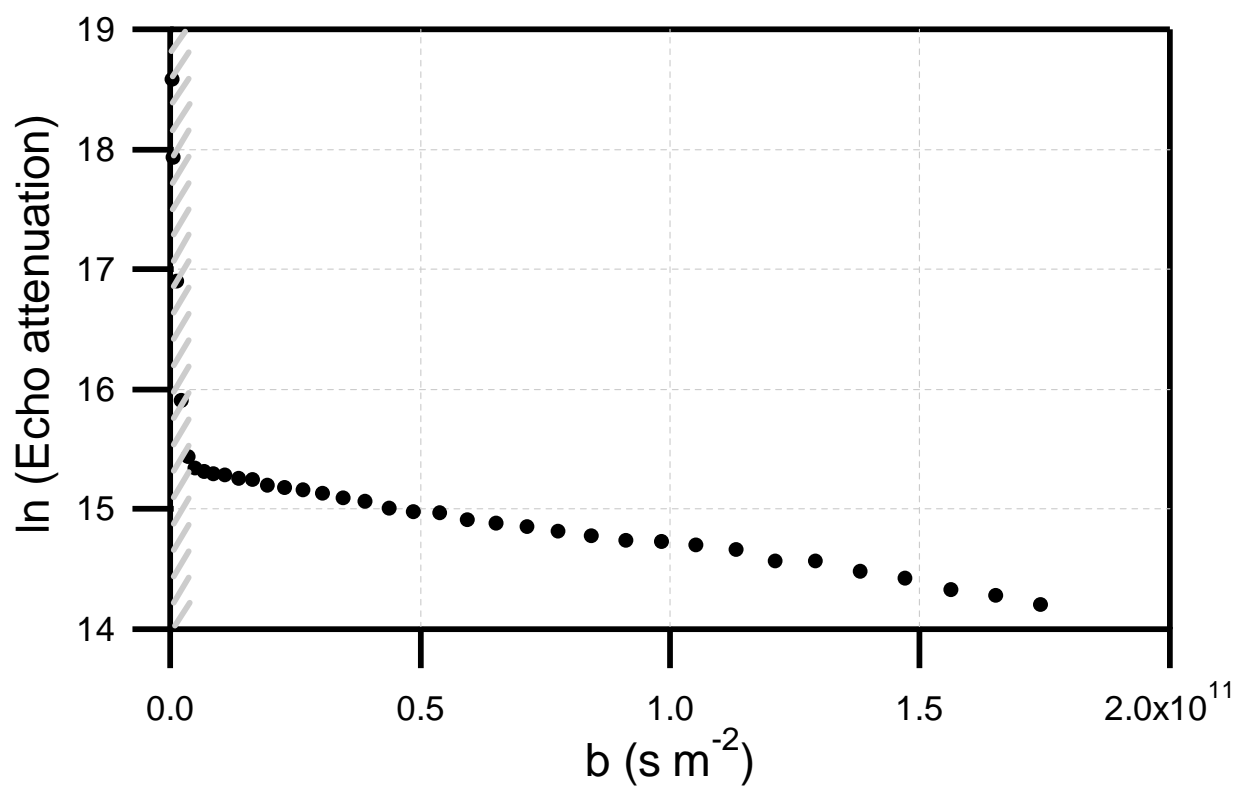

Figure 3.4: A representative echo attenuation plot for casein aggregates in Na-caseinate dispersion (6 $\mathrm{wt} \%)$. Note that $\mathrm{b}$ is $\gamma^{2} g^{2} \delta^{2}(\Delta-\delta / 3)$.

In Fig. 3.5, the so-obtained casein aggregate diffusion coefficient is plotted as a function of Na-caseinate concentration, for observation times of 20,60 and $140 \mathrm{~ms}$ between the two gradient pulses $(\Delta)$. At low Na-caseinate concentration the measured diffusion coefficient is constant, within uncertainty as a function of Na-caseinate concentration. This is as expected if unrestricted diffusion is being probed, given that the size distribution does not change upon increasing Na-caseinate concentration. Further increasing the concentration $(>4$ $w t \%$ Na-caseinate) results in a rapid decrease in the casein aggregates' diffusion coefficient. The rate of change slows down considerably once approximately $10 \mathrm{wt} \%$ Na-caseinate is reached. Both of these transitions coincide with observed changes in the visual appearance of the sample (turbidity and flow) at $\sim 6$ and $\sim 10 \mathrm{wt} \%$. Moreover, upon increasing observation time we note that for concentrations $>4 \mathrm{wt} \% \mathrm{Na}$-caseinate the measured ensemble average diffusion coefficients decrease, indicating that restricted diffusion is measured at these longer times. In contrast for the low concentration samples unrestricted diffusion is always measured. 


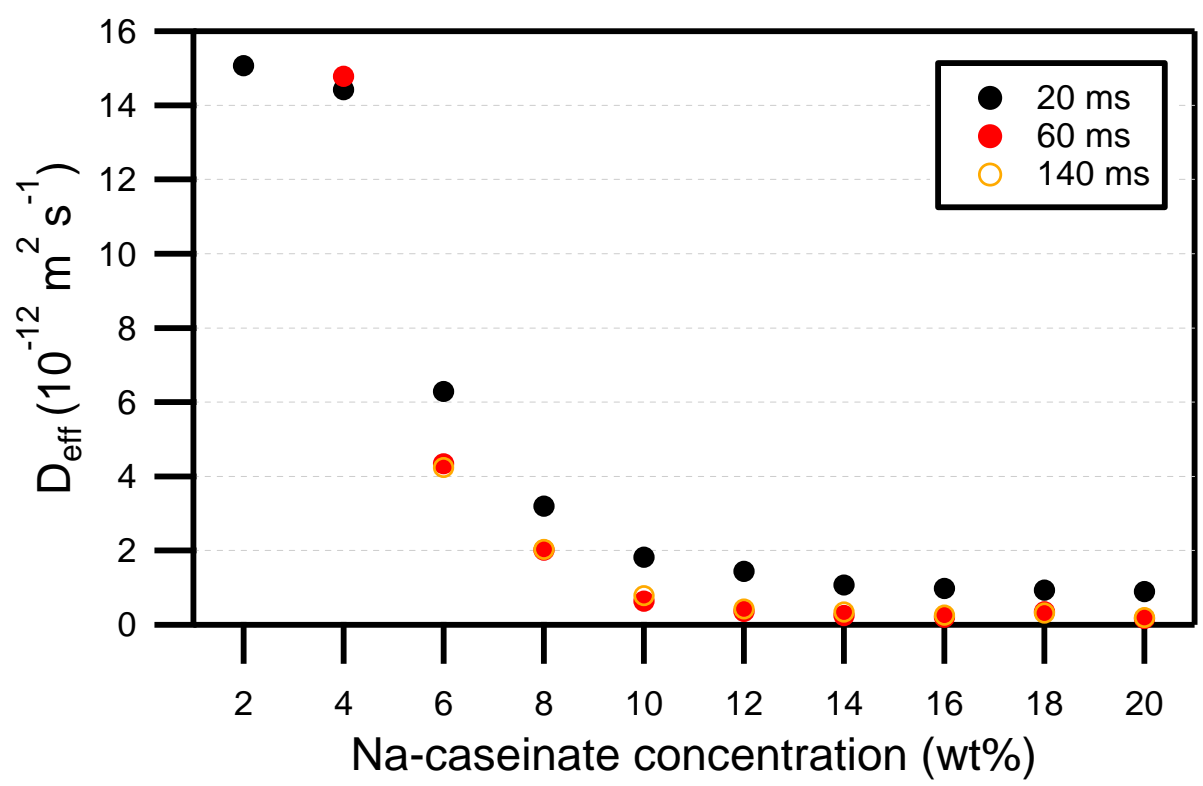

Figure 3.5: Observed casein aggregate diffusion coefficients as a function of Na-caseinate concentration for Nacaseinate dispersions, $\Delta=20,60$ and $140 \mathrm{~ms}$. Note that for the $2 \mathrm{wt} \%$ sample data at the longer observation times was unreliable due to the low signal-to-noise and poor attenuation of the signal.

It was not possible to probe all observation times for all Na-caseinate concentrations. As Na-caseinate concentration was decreased the signal-to-noise ratio was significantly reduced and the extent of signal attenuation was insufficient as $\Delta$ was increased. As such only short observation times returned reliable attenuation data for low Na-caseinate concentration. Moreover the range of observation times available was considerably restricted due to the short $T_{1}$ values of the casein aggregates. $T_{1}$ was estimated to be between 0.4 and $1.3 \mathrm{~s}$, as measured for a Na-caseinate dispersion in $\mathrm{D}_{2} \mathrm{O}$ using a high resolution $600 \mathrm{MHz}$ Varian spectrometer. Given that $T_{1}$ is in the range of hundreds of ms, $\Delta$ was restricted to a maximum of $180 \mathrm{~ms}$. This coupled with the extremely weak signal meant that we were unable to probe more fully the restricted and unrestricted diffusion of the aggregates. Despite these limitations analysis of the data obtained for values of $\Delta$ in the range of 12 to $180 \mathrm{~ms}$ indicated that for values of $\Delta<40 \mathrm{~ms}$ unrestricted diffusion was probed for all concentrations and that for $\Delta>40 \mathrm{~ms}$ restricted diffusion was probed for concentrations of $6 \mathrm{wt} \% \mathrm{Na}-$ caseinate and higher. 


\subsubsection{Rheology}

Non-linear rheology measurements could not be performed on samples below $8 \mathrm{wt} \%$ as the viscosity of the samples was too low, being on the order of that of water or above $12 \mathrm{wt} \%$ as the viscosity was too high. As such the results and discussion given below are for samples of 8 to $12 \mathrm{wt} \%$ Na-caseinate.

The stress response of the materials to an applied constant shear rate as demonstrated by a $10 \mathrm{wt} \%$ sample follows three distinctly different behaviours (Fig. 3.6). At start up, $t=0.01$ $\mathrm{s}$, the stress rises steadily before settling down to a constant value (within $0.05 \mathrm{~s}$ ). The plateau is maintained over a long time with small periodic changes evident (see inset of Fig. 3.6). This constant stress regime increases with shear rate. At longer times $\left(>10^{4} \mathrm{~s}\right)$ a sharp increase in stress is observed, due to gelation of the sample. The aged sample is significantly more viscous compared to the fresh. It must be noted that prior to the onset of gelation all behaviour is reversible. The gelation process however is irreversible. The onset of the gelation process is shear rate dependent as shown in the normalised data of Fig. 3.7. The data was normalised using the average stress value in the plateau region seen in Fig. 3.6 (and Fig. 3.9), separately for each curve. As the shear rate is increased, the gelation process occurs at a much slower rate. No change is observed in the initial start up region. The increase in stress on increasing shear rate shown in Fig. 3.8 at different shearing times beyond the plateau region is essentially a linear function in relationship.

The long-time stress response of different $\mathrm{Na}$-caseinate dispersions at a constant shear rate $\left(100 \mathrm{~s}^{-1}\right)$ are displayed in Fig. 3.9. In order to obtain reliable data, high shear rates needed to be applied to the low concentration samples and vice versa for higher concentration samples. As such, the shear rate of $100 \mathrm{~s}^{-1}$ was chosen to study the stress evolution of Nacaseinate dispersions as a function of $\mathrm{Na}$-caseinate concentration as this ensured data for all concentrations could be obtained at the same shear rate. The onset of the shear-induced gelation process is concentration dependent; the higher the Na-caseinate concentration, the faster gelation occurs (Fig. 3.10). The stress response on increasing Na-caseinate concentration, at $100 \mathrm{~s}^{-1}$, is an increasing non-linear function (Fig. 3.11). The $8 \mathrm{wt} \% \mathrm{sample}$ returns a near constant stress value up to $\sim 20000 \mathrm{~s}(\sim 5.5 \mathrm{hrs})$, i.e., no gelation occurs over this time, while for samples with $11 \mathrm{wt} \% \mathrm{Na}$-caseinate and above a dramatic increase in stress is observed. At $12 \mathrm{wt} \%$ Na-caseinate for example, gelation begins within $\sim 500 \mathrm{~s}$. This corresponds to a near 40-fold reduction in gelation time for only a 1.5 times increase in $\mathrm{Na}$ - 
caseinate concentration. The short-time response $(<0.05 \mathrm{~s})$ of the samples is shear rate (Fig. 3.7) and concentration (Fig. 3.10) independent.

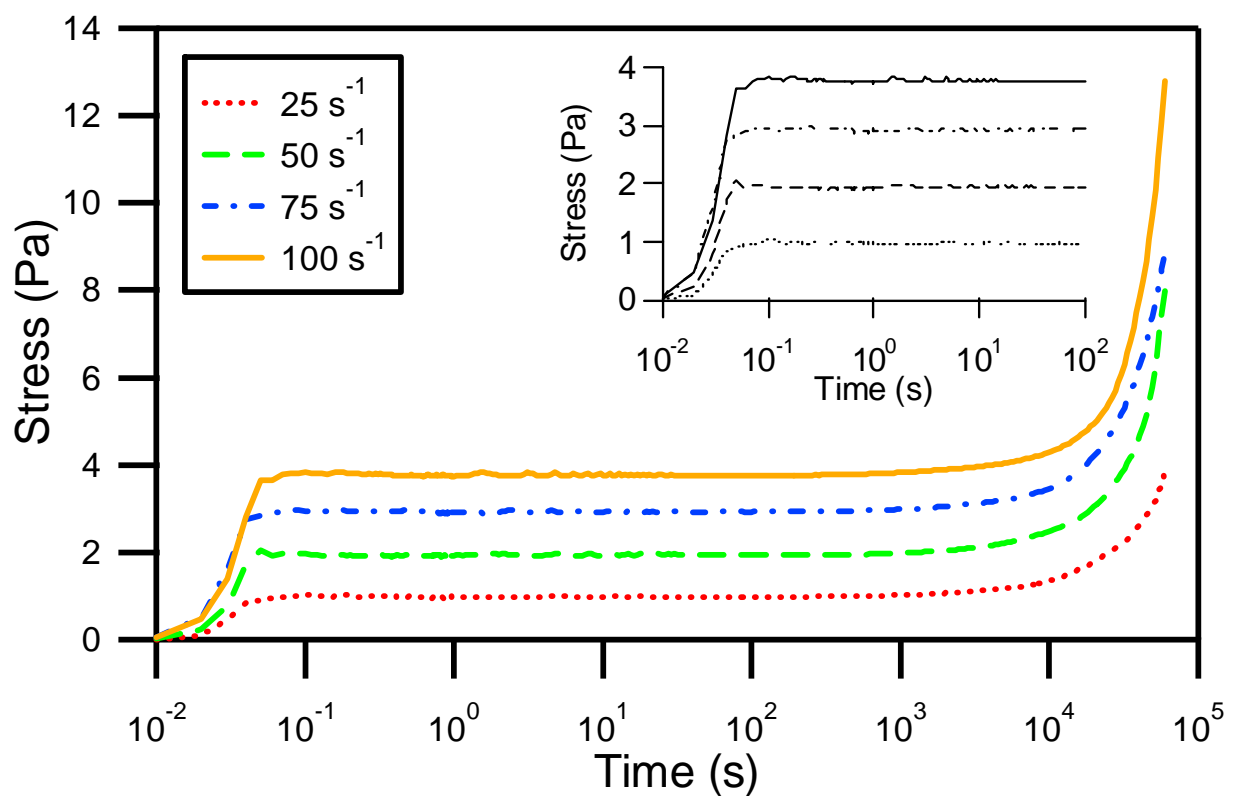

Figure 3.6: Lin-log plot of the experimental stress response as a function of time at different shear rates for a 10 $\mathrm{wt} \% \mathrm{Na}$-caseinate dispersion.

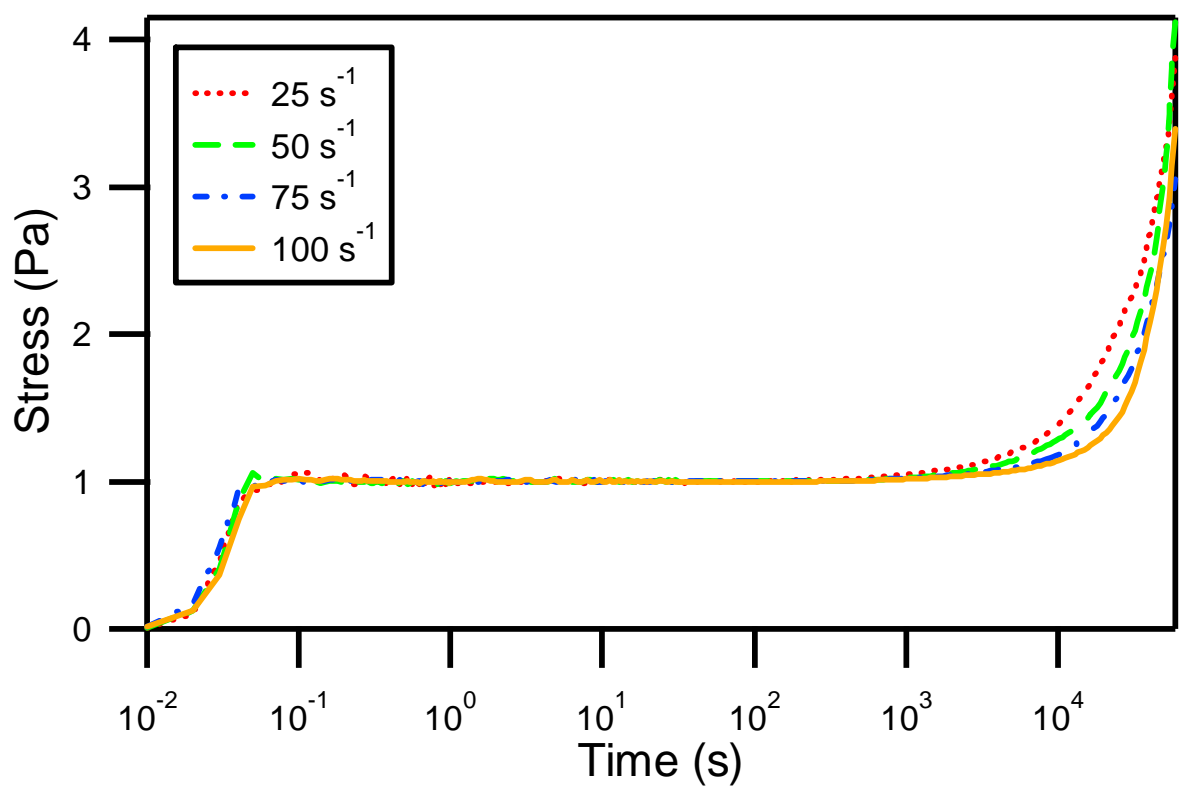

Figure 3.7: Normalised stress response data for a $10 \mathrm{wt} \%$ Na-caseinate dispersion (data was normalised to the average stress value in the plateau region in Fig. 3.6 for each shear rate). 


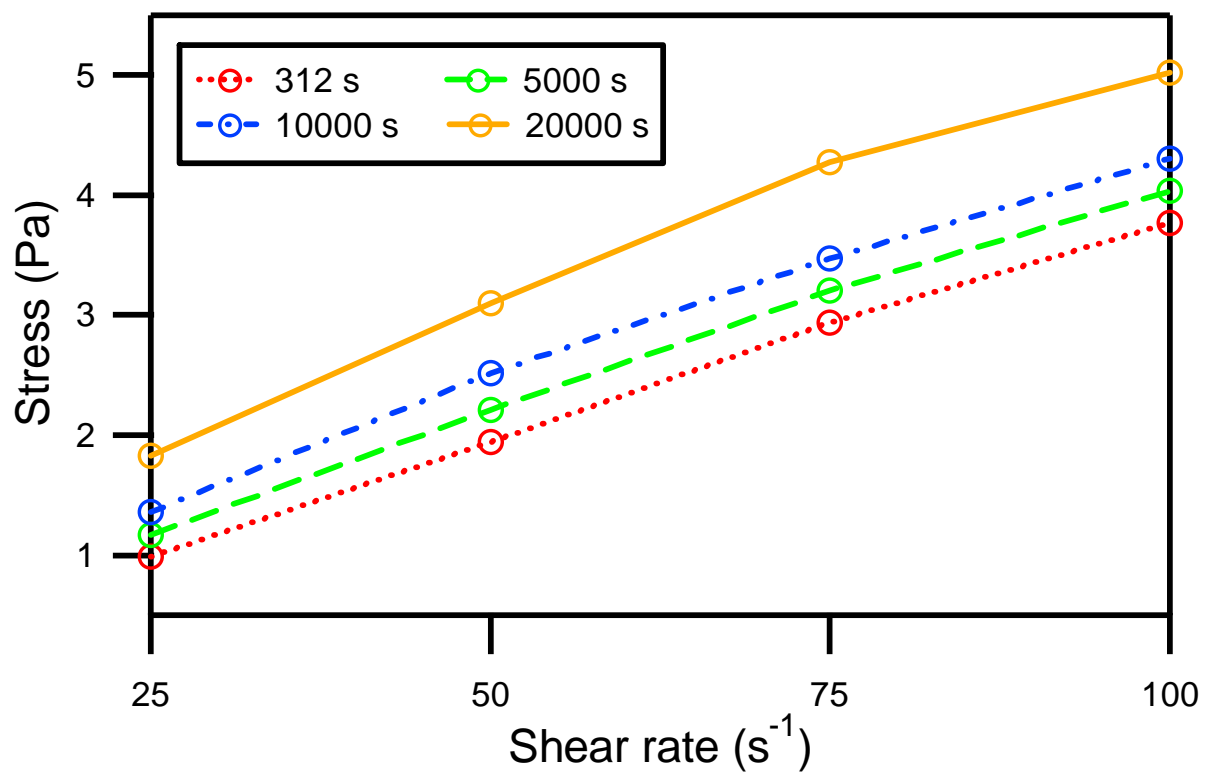

Figure 3.8: Experimental stress value as a function of shear rates (data taken at different times from Fig. 3.6).

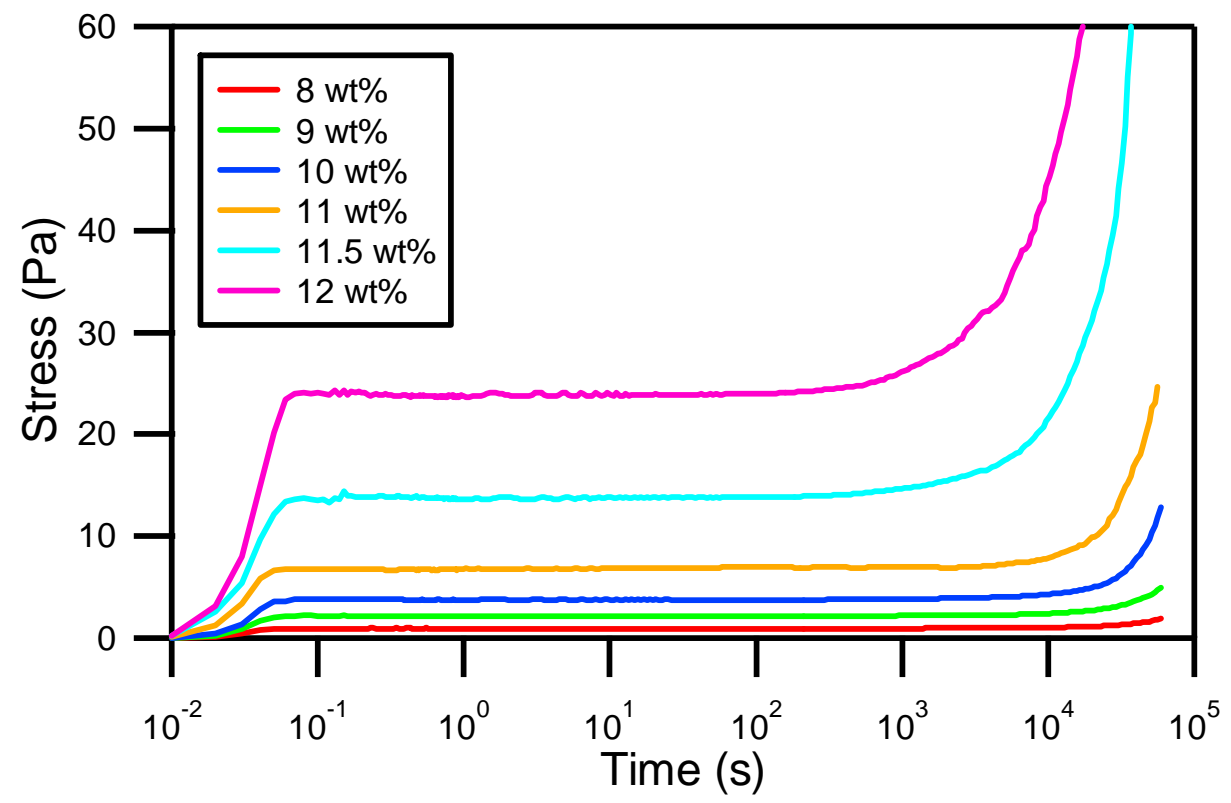

Figure 3.9: Lin-log plot of the experimental stress response as a function of time, at $\dot{\gamma}=100 \mathrm{~s}^{-1}$ for six different $\mathrm{Na}$-caseinate concentrations. 


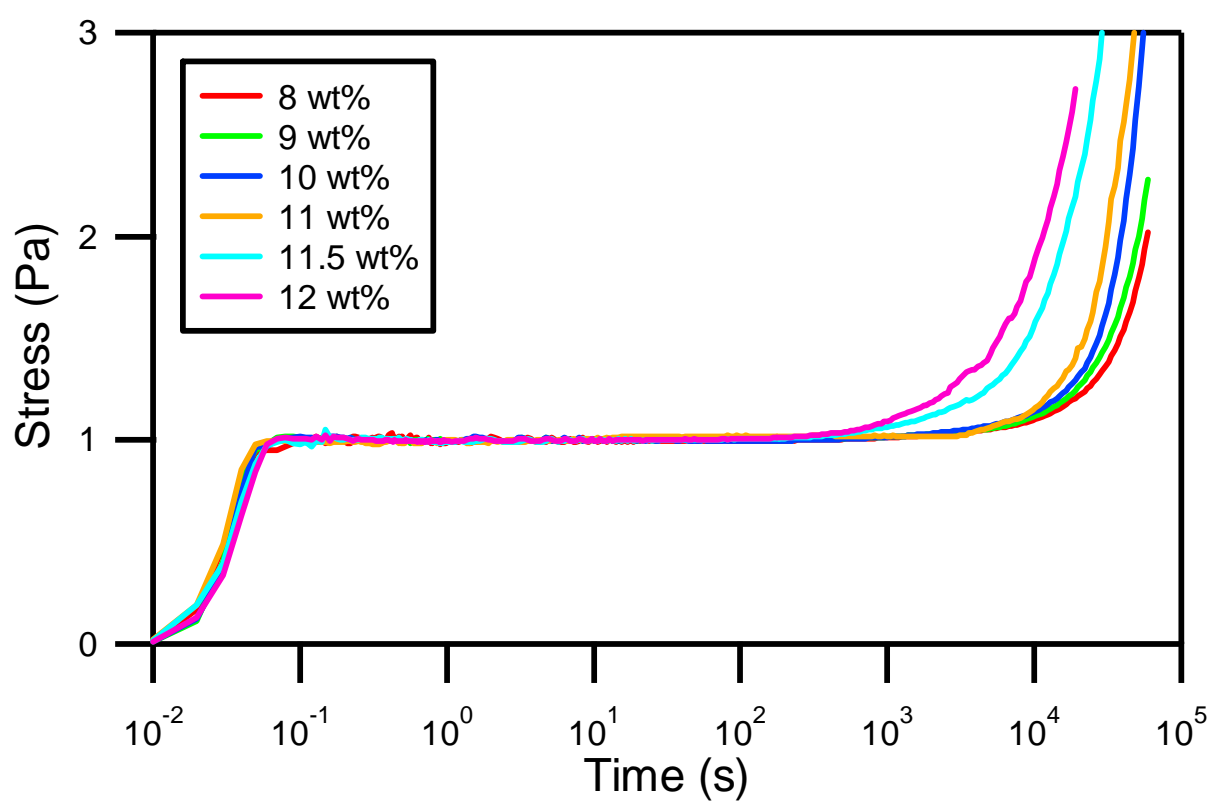

Figure 3.10: Normalised stress response data (normalised to the average stress value in the plateau region in Fig. 3.9 for each curve).

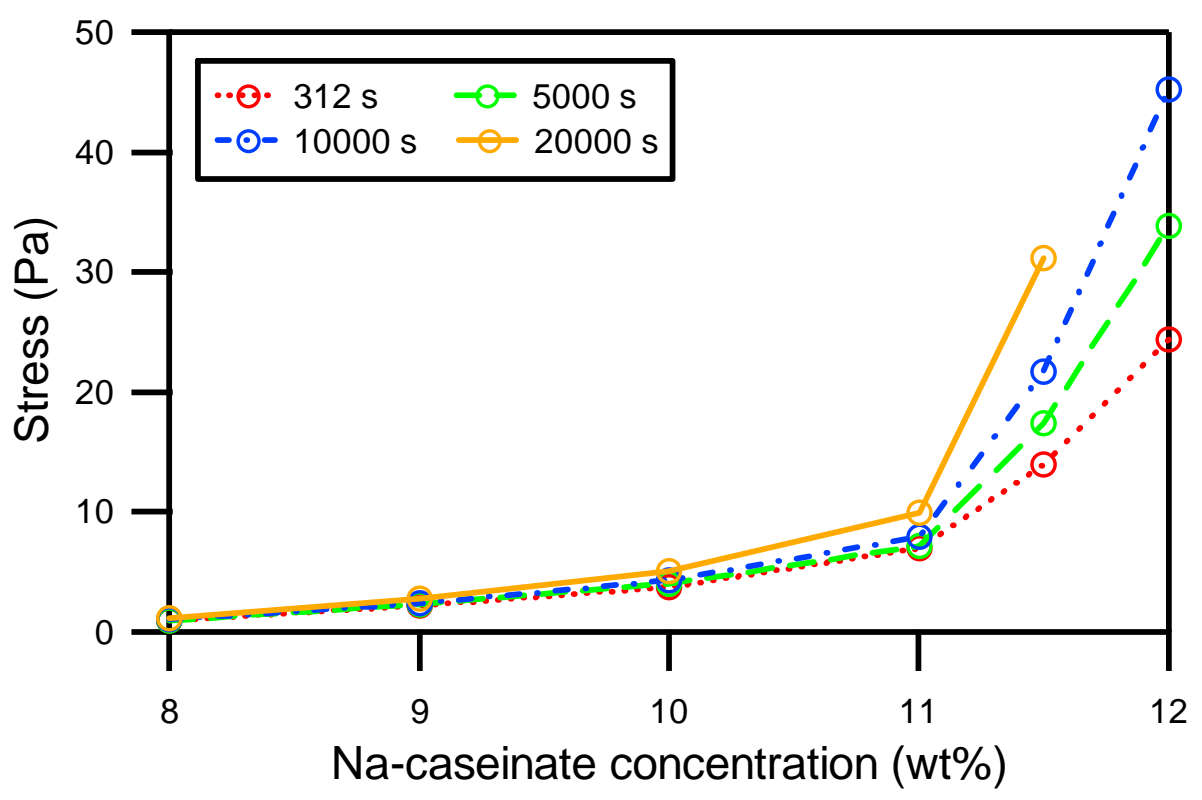

Figure 3.11: Experimental stress values as a function of Na-caseinate concentration (data taken at different times from Fig. 3.10).

Linear viscoelastic measurements could not be performed on samples below $10 \mathrm{wt} \%$ as the viscosity of the samples was too low. The oscillatory strain response of different concentration samples at a constant angular frequency is given in Fig. 3.12. It can be seen that $G^{\prime \prime}$ always lies above $G^{\prime}$ for each sample indicating the material behaves as a liquid under these conditions. $G^{\prime}$ and $G^{\prime \prime}$ do not depend on the deformation applied to the system and they 
are parallel to each other over several orders of magnitude in strain. As the concentration of Na-caseinate is increased, the maximum possible strain able to be applied decreased; higher imposed strains resulting in unreliable data. Using a stress-controlled rheometer (AR2000) data at higher strain amplitude were obtained corresponding to the first indication of entry into the non-linear response regime, however no cross over was observed (Fig. 3.13). In Fig. 3.14 is shown the calculated complex modulus $G^{*}$ as a function of Na-caseinate concentration: a steady monotonic increase is evident. The more than two orders of magnitude increase in $G^{*}$ on increasing Na-caseinate concentration from 12 to $18 \mathrm{wt} \%$ indicates that the concentrated samples typically have gel-like rheology (a high $G^{*}$ value) while being locally fluid.

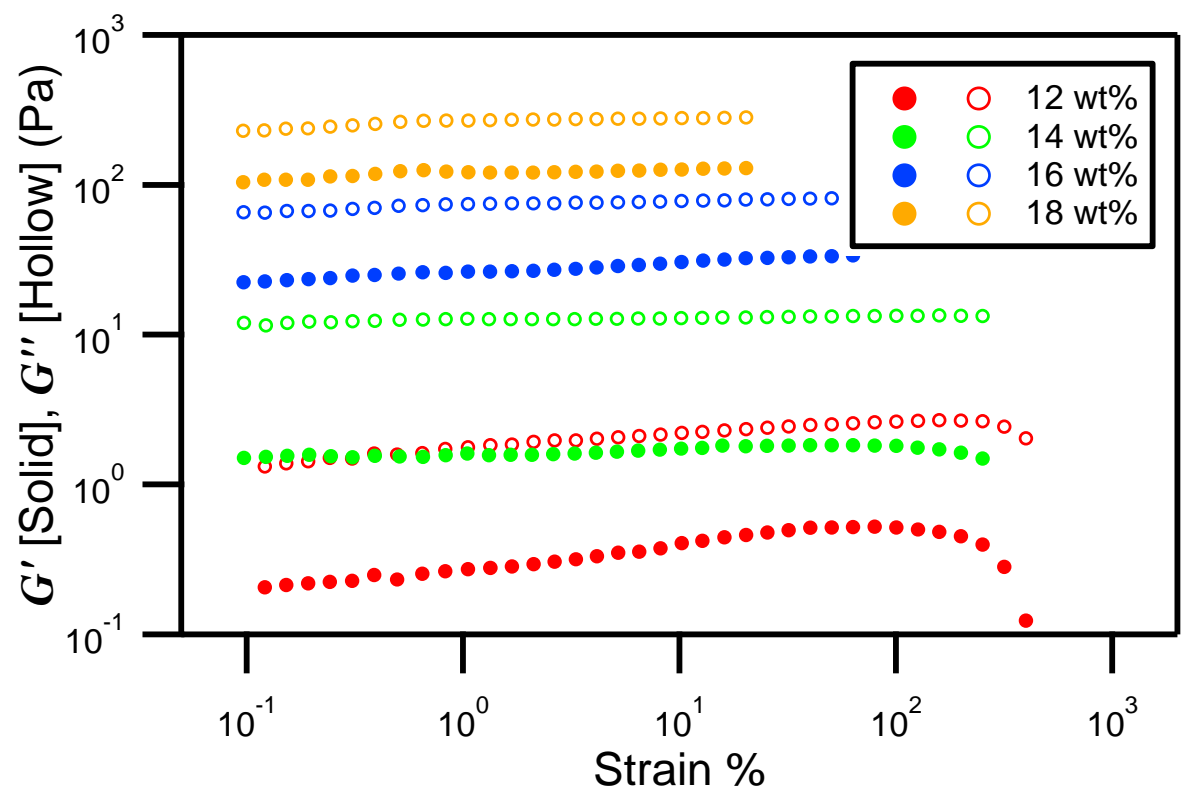

Figure 3.12: Log-log plot for the dynamic strain sweep at $1 \mathrm{rad} \mathrm{s}^{-1}$ (strain-controlled rheometer) for 12 to 18 $\mathrm{wt} \% \mathrm{Na}$-caseinate dispersions. 


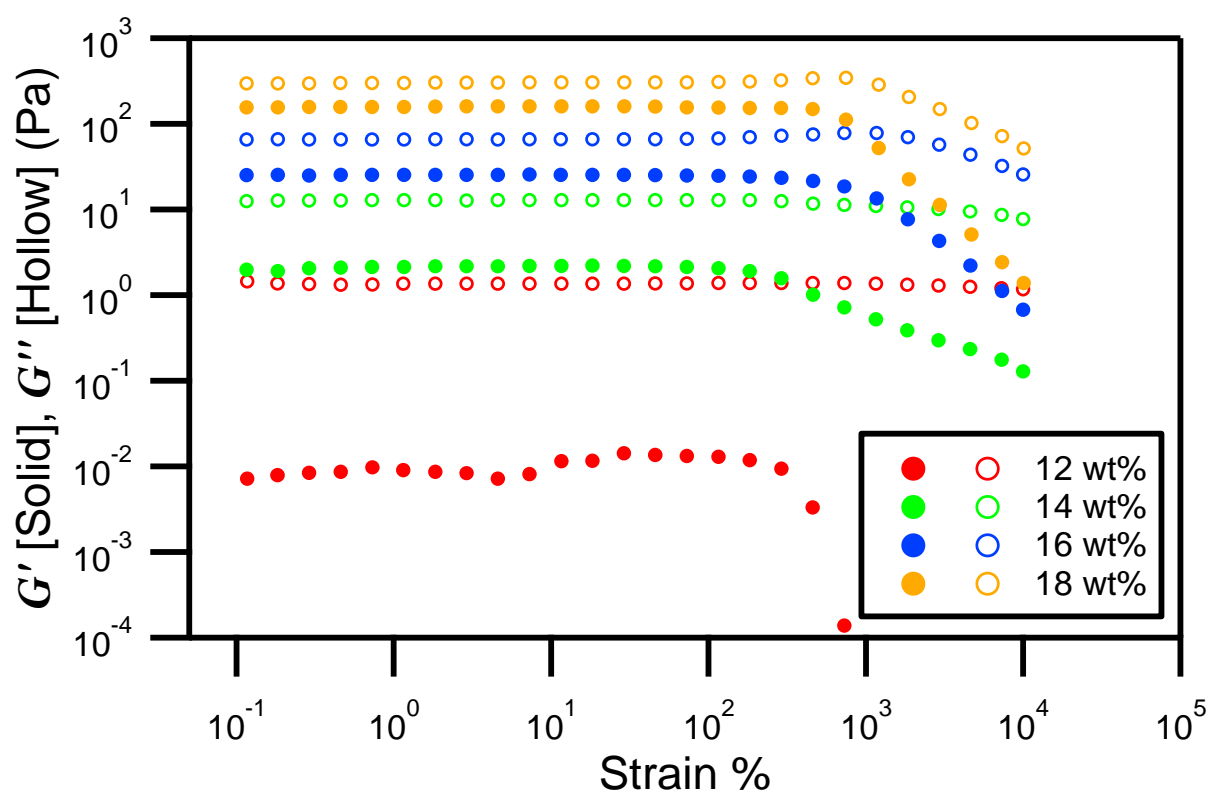

Figure 3.13: Log-log plot for the dynamic strain sweep at $1 \mathrm{rad} \mathrm{s}^{-1}$ (stress-controlled rheometer) for 12 to 18 $\mathrm{wt} \%$ Na-caseinate dispersions.

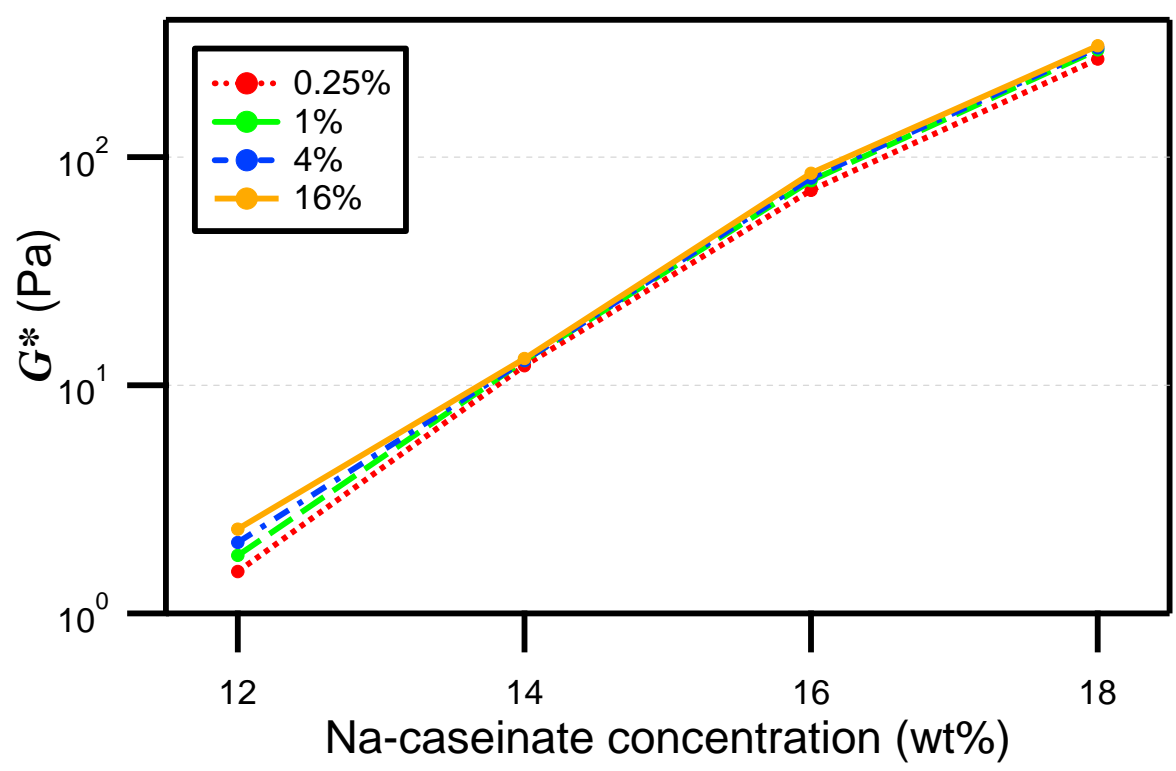

Figure 3.14: Calculated complex modulus $\left(G^{*}\right)$ variation for 12 to $18 \mathrm{wt} \%$ Na-caseinate dispersions (data taken from Fig. 3.12 at different strain amplitude).

Fig. 3.15 is a plot of the frequency response of the samples to an oscillatory shear of $1 \%$ strain amplitude. $G^{\prime}$ and $G^{\prime \prime}$ show a strong dependence on concentration. $G^{\prime \prime}$ is greater than $G^{\prime}$ for the $16 \mathrm{wt} \%$ Na-caseinate sample and below whereas a clear cross over is seen in the frequency sweep of the $18 \mathrm{wt} \%$ Na-caseinate sample (the sample becomes more solid-like at higher frequency). All samples show a tendency towards such a cross over due to the non- 
parallel variation of $G^{\prime}$ and $G^{\prime \prime}$. Reliable data above $10 \mathrm{~Hz}$ could not be obtained.

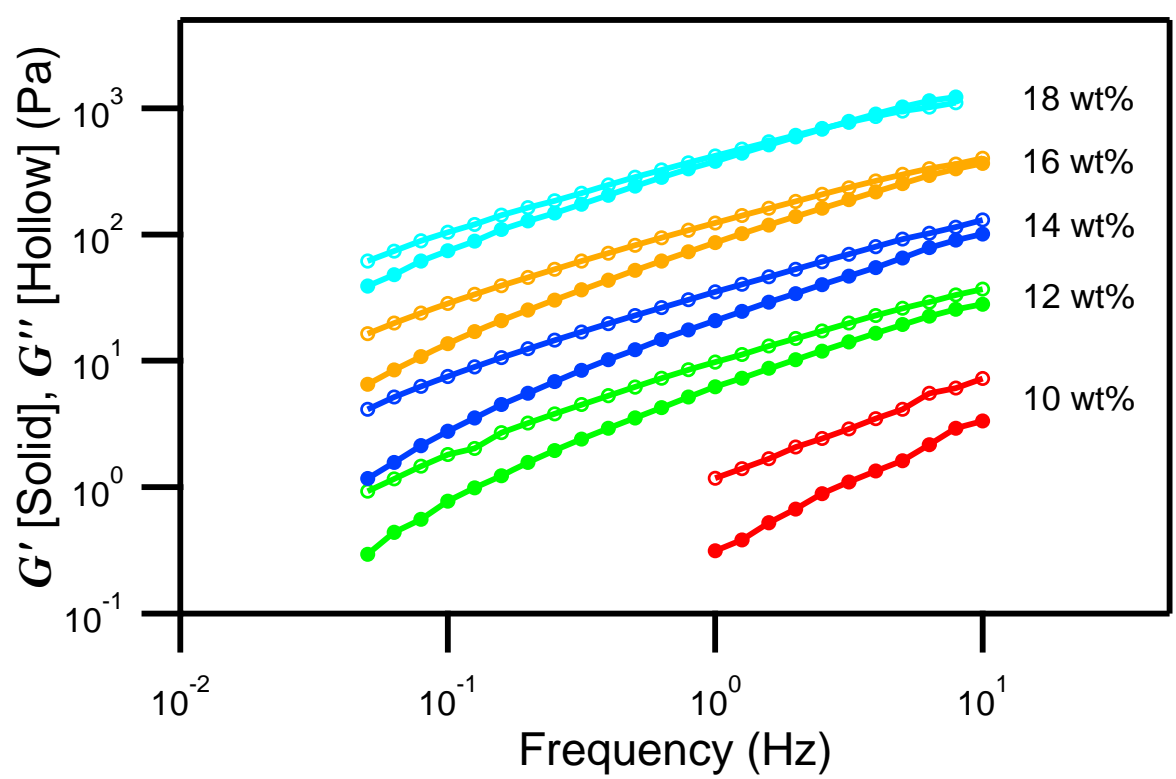

Figure 3.15: Log-log plot of the dynamic frequency sweep at $1 \%$ strain amplitude for 10 to $18 \mathrm{wt} \%$ Na-caseinate dispersions.

The evolution of the dynamic modulus when submitting a $12 \mathrm{wt} \%$ Na-caseinate dispersion to an oscillatory shear at an angular frequency of $\omega=1 \mathrm{rad} \mathrm{s}^{-1}$ with a strain amplitude of 5\% (linear response regime) is shown in Fig. 3.16. Initially, $G^{\prime}$ and $G^{\prime \prime}$ are constant over a long period of time. At longer times, there is a marked increase in both components of the modulus until the solid-like characteristic dominates the aged sample $\left(G^{\prime}\right.$ $\left.>G^{\prime \prime}\right)$. The transition to a stiffer state, characterised by an increase in the storage modulus, $G^{\prime}$ coupled with a reduction in fluidity $(\tan \delta \rightarrow 0)$ occurs at $\sim 10^{4} \mathrm{~s}$. This complies with the time dependent stress response data for the lower concentration samples (Fig. 3.6). Within the experimental time of $60000 \mathrm{~s}$, the characteristic time of the onset of $G^{\prime}>G^{\prime \prime}$ for 11 to 18 $\mathrm{wt} \% \mathrm{Na}$-caseinate dispersions varies from approximately 23000 to $49000 \mathrm{~s}$ with no particular trend observed on increasing $\mathrm{Na}$-caseinate concentration. 


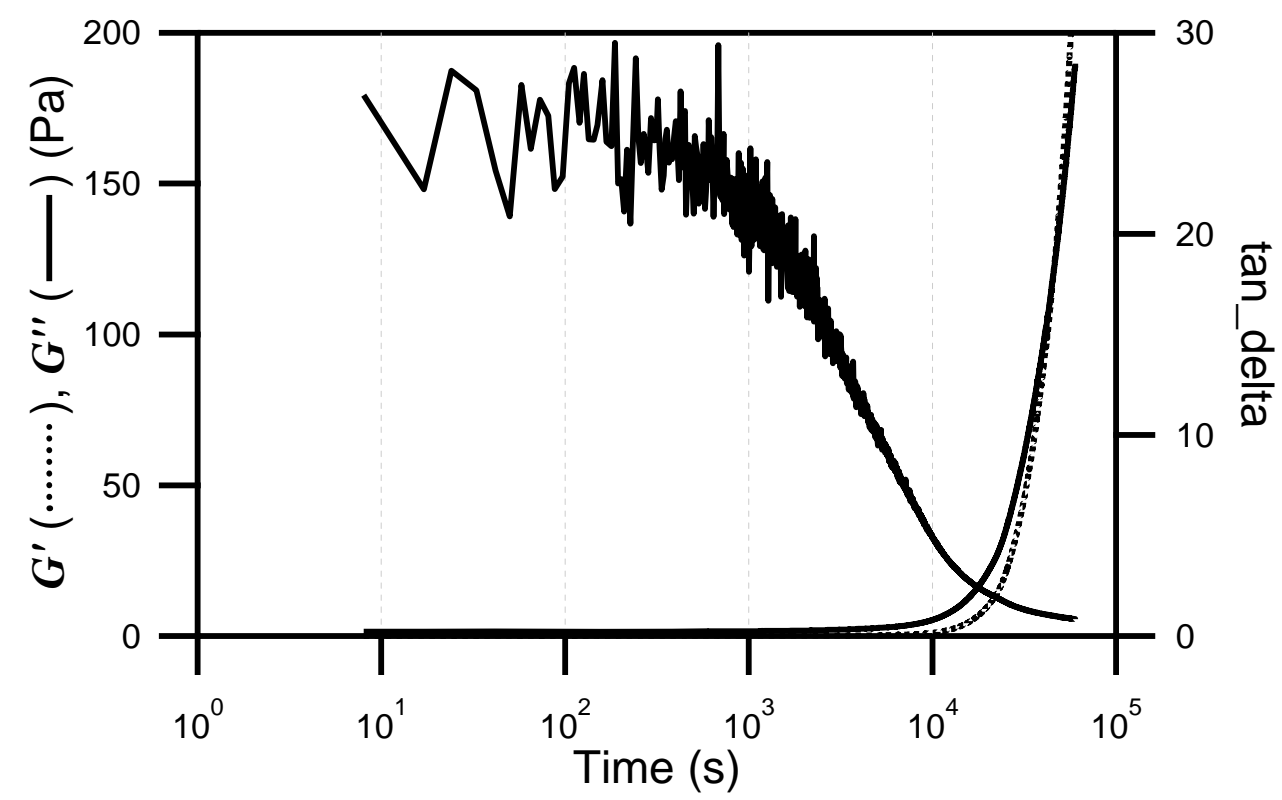

Figure 3.16: Lin-log plot of the dynamic time sweep at the frequency of $1 \mathrm{rad} \mathrm{s}^{-1}$ and $5 \%$ strain amplitude (linear response regime) for $12 \mathrm{wt} \% \mathrm{Na}$-caseinate dispersion.

\subsection{Discussion}

\subsubsection{PGSTE-NMR}

The diffusion coefficient of water decreases on increasing Na-caseinate concentration (Fig. 3.3). A reduction in the diffusion coefficient is expected due to the presence of an increasing number of casein aggregates. As a first approximation this decrease can be attributed to increasing obstruction effects induced by the slow-moving casein aggregates which are assumed to be impenetrable. The theory of Wang ${ }^{12}$ and Jönsson et al. ${ }^{13}$ can be evoked where

$$
\frac{D_{\text {eff }}}{D_{0}}=\frac{1}{1+\varphi / 2}
$$

$\frac{D_{e f f}}{D_{0}}$ is the obstruction factor for a system containing monodisperse spherical aggregates. $D_{\text {eff }}$ is the effective diffusion coefficient of the continuous phase, $D_{0}$ is the diffusion coefficient for the bulk liquid and $\phi$ is the volume fraction of the aggregates $\left(0.75 \mathrm{~cm}^{3} \mathrm{~g}^{-1}\right.$ and $1 \mathrm{~cm}^{3} \mathrm{~g}^{-1}$ for casein and water, respectively) $)^{6}$. Apart from the obstruction effect, the model also considers hydration and shape factors. 
Applying this model to our system we find that the calculated values of $D_{\text {eff }}$, based on known volume fractions, are up to two times larger than the measured water diffusion coefficients. The breakdown of the theory is in part due to the microstructure of our Nacaseinate dispersions not complying with that of a uniform distribution of spherical aggregates in a fluid continuum (Fig. 3.2). At a minimum therefore the effect of polydispersity must be considered. More importantly however is the possibility of interaction between the water molecules and the casein aggregates (i.e., the hydration effect), water penetration into the casein aggregates, and/or interaggregate interactions. All will contribute to a reduction in the water diffusion coefficient.

Consider an observation time, $\Delta$ of $20 \mathrm{~ms}$. The average distance probed by a water molecule in this time is at least $7 \mu \mathrm{m}$, which is very large compared to the average diameters of the casein aggregates $(0.133 \mu \mathrm{m})$. On increasing concentration, the number of aggregates in the solution increases. The interaction between casein aggregates becomes more pronounced and thus the movement of the water molecules becomes severely restricted. The travelling pathway of the water molecules now begins to dominate the rate at which the water molecules can move. Hence an increase in tortuosity ${ }^{14-16}$ will decrease the measured diffusion coefficient for water.

Two models have been used to describe water mobility in casein aggregates (see 10 in which both casein aggregates and native casein micelles were investigated for a detailed description).

The first model is applied to Na-caseinate concentrations less than the close packing limit, $\sim 10 \% \mathrm{w} / \mathrm{v}^{17}$. On a macroscopic level, this model assumes that there are two different water regions, one of which is inside (i.e., water that has penetrated into the casein aggregates) and the other is surrounding the spherical aggregates (water interacting with the periphery of the casein aggregates). Obstruction effects and water-aggregate interactions manifest themselves in the values of the diffusion coefficients in these regions. Despite there being several different sites at which the water diffuses at different rates, such inhomogeneities are averaged out when the exchange of water molecules between the regions is rapid, as is the case here. The contribution of specific water-protein "binding" in lowering the water mobility is small (a few water molecules/aggregate) and can be negligible ${ }^{18}$. Thus, the effective water diffusion coefficient at low Na-caseinate concentration (2 to $8 \mathrm{wt} \%$ ) is given by 


$$
D_{\text {eff }}^{\text {water }}=2.3 \times 10^{-9}\left(1+0.75 \frac{m^{\text {cas }}}{m^{\text {water }}}\right) \frac{\left(1+0.75 \frac{m^{\text {cas }}}{m^{\text {water }}}-K \frac{m^{\text {cas }}}{m^{\text {water }}}\right)}{\left(1+0.75 \frac{m^{\text {cas }}}{m^{\text {water }}}+K \times 0.5 \frac{m^{\text {cas }}}{m^{\text {water }}}\right)}
$$

with $K=\left(v^{\text {cas }} / v^{\text {water }}+H^{\text {cas }}\right) \beta$ and $\beta=\frac{D_{2} C_{2}-D_{1} C_{1}}{D_{2} C_{2}+0.5 D_{1} C_{1}}$ where $m^{\text {cas }}$ and $m^{\text {water }}$ are the mass of casein and water $(\mathrm{g}), v^{\text {cas }}$ and $v^{\text {water }}$ are the specific volume of casein and water $\left(\mathrm{cm}^{3} \mathrm{~g}^{-1}\right)$, $H^{\text {cas }}$ is the amount of water in the casein micro-gel (grams of water per gram of casein), $C_{1}$ and $D_{1}$ are the water concentration and diffusion coefficient inside the aggregate, $C_{2}$ and $D_{2}$ are the water concentration and diffusion coefficient in the region surrounding the aggregate, $K$ can be obtained by fitting the equation to experimental data. The result of the fitting process is presented in Fig. 3.17, with $K=2.13$.

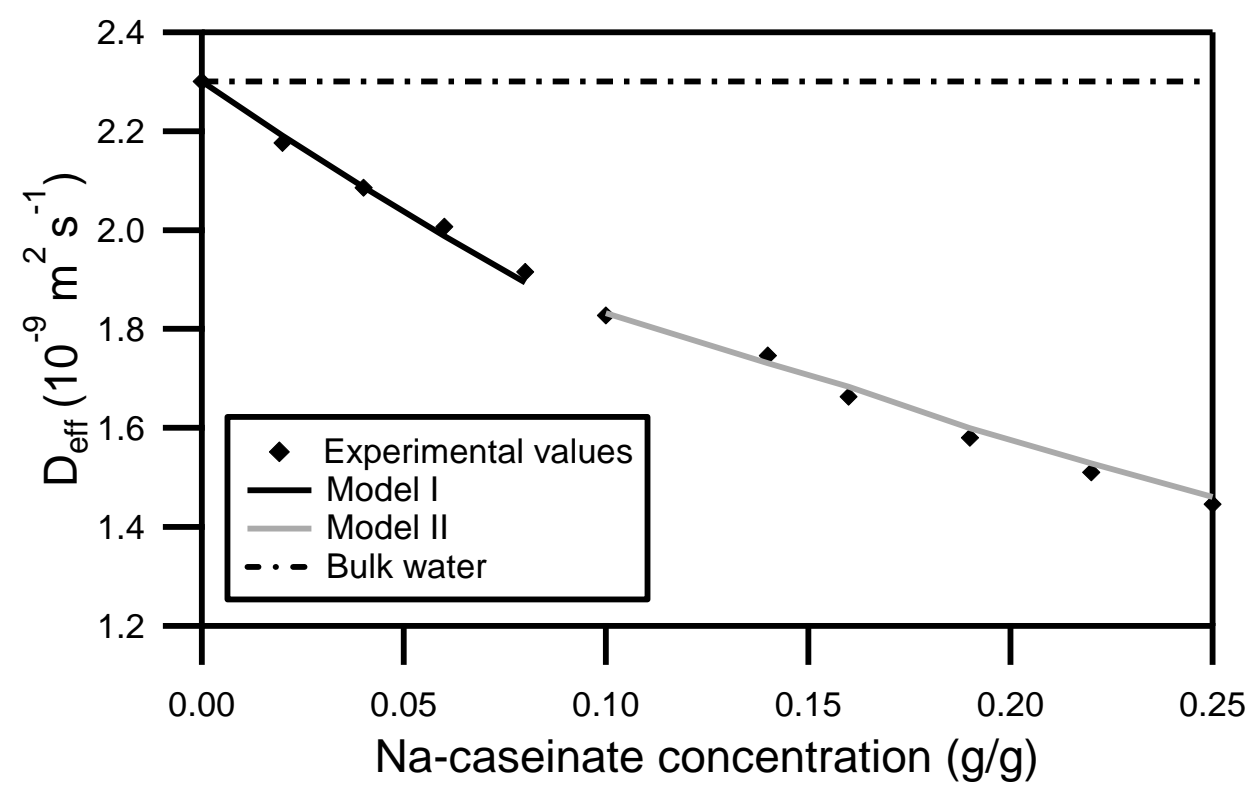

Figure 3.17: Variation of the water diffusion coefficient $D_{\text {eff }}$ as a function of Na-caseinate concentration (2 to 8 $\mathrm{wt} \%$ ). The line corresponds to the fit from equation 3.3 with average $K$ value $=2.13$.

In the second model, the aggregate is characterised by water-rich regions in a waterpoor matrix. A water molecule needs to diffuse through a water-poor matrix when travelling from one water-rich region to another. The effective water diffusion coefficient at higher Nacaseinate concentration (10 to $20 \mathrm{wt} \%$ ) becomes

$$
D_{\text {eff }} \approx 2.3 \times 10^{-9}\left(\frac{1}{1+2.3 \times m_{\mathrm{c}} / m_{\mathrm{w}}}\right)
$$


where $m_{c} / m_{w}$ is the $g_{\text {casein }} / g_{\text {water }}$. The concentration dependence implied by equation 3.4 is plotted in Fig. 3.17.

Turning to the casein aggregate diffusion data we see in Fig. 3.5 that the measured ensemble average diffusion coefficient decreases on increasing Na-caseinate concentration. The two transition points we observed, i.e., at $>4 \mathrm{wt} \%$ and $\sim 10 \mathrm{wt} \%$ Na-caseinate, are in good agreement with results published by Farrer et al. where their study concerned the onset of close packing of the submicelles via osmotic pressure measurement at $\geq 30^{\circ} \mathrm{C}^{17}$.

Analysis of the measured diffusion coefficients as a function of experimental observation time for each of the concentrations investigated reveals that for $\Delta=20 \mathrm{~ms}$ unrestricted diffusion of the aggregates is measured for all concentrations ( 2 to $20 \mathrm{wt} \%$, this is evidenced since the measured diffusion coefficients at a given $w \mathrm{t} \%$ are constant over a range of observation times, this range diminishing with increasing Na-caseinate concentration). On the face of it this information indicates that on average the casein aggregates increase in size on increasing Na-caseinate concentration. From the light scattering data however we know that for all Na-caseinate dispersions a near identical aggregate size distribution is returned with the only modification on increasing Na-caseinate concentration being a slight augmentation of the larger aggregate size tail of the distribution. This larger tail is most likely being due to the formation of larger aggregates, i.e., an increased contribution of interaggregate interactions.

To understand this apparent discrepancy one must take into consideration that NMR is a volume-based technique, even a slight increase in the large aggregate size tail of the size distribution can falsely represent the diffusion of the larger droplets in the PGSTE-NMR data. This will therefore lead to the anomalous conclusion that the average aggregate size increases with increasing Na-caseinate concentration.

In analysing the data we may start from the view point that for $\Delta=20 \mathrm{~ms}$ we are measuring unrestricted diffusion for all Na-caseinate concentrations. Thus the StokesEinstein equation may be applied to estimate the average diameter at infinite dilution using,

$$
D=\frac{k T}{6 \pi \eta r}
$$

where $D$ is the diffusion coefficient of the aggregates, $r$ is the hydrodynamic radius of the aggregate, $k$ is the Boltzmann's constant, $T$ is the absolute temperature and $\eta$ is the viscosity of the continuous phase $\left(0.89 \mathrm{mPa} \mathrm{s}\right.$ at $25^{\circ} \mathrm{C}$ for water). Table 3.1 summarises the calculated average aggregate diameter as estimated from equation 3.5. 
Table 3.1: Calculated average diameter of aggregates in Na-caseinate dispersions

\begin{tabular}{lccccccccccc}
\hline & \multicolumn{10}{c}{ Na-caseinate concentration (wt\%) } \\
& 2 & 4 & 6 & 8 & 10 & 12 & 14 & 16 & 18 & 20 \\
\hline $\begin{array}{l}\text { diff. coeff. of } \\
\text { caseinate, } 10^{-12} \mathrm{~m}^{2} \mathrm{~s} \mathrm{~s}^{-1}\end{array}$ & 15.06 & 14.42 & 6.29 & 3.20 & 1.82 & 1.44 & 1.07 & 0.98 & 0.93 & 0.90 \\
calculated $d(\mu \mathrm{m})$ & 0.033 & 0.034 & 0.078 & 0.153 & 0.269 & 0.341 & 0.458 & 0.499 & 0.528 & 0.545 \\
\hline
\end{tabular}

While a direct correlation between the measured casein aggregate diffusion coefficient and the average aggregate diameter, through Stokes-Einstein equation, is not strictly applicable here, the calculated $d$ values shown in Table 3.1 span the aggregate size distribution shown in Fig. 3.2 (as expected based on the above discussion). Moreover, they clearly show that at higher Na-caseinate concentrations we are predominantly probing the high end of the size distribution using PGSTE-NMR.

The calculated values of the aggregate diameters given in Table 3.1 are in agreement with previously published results ${ }^{19}$. These authors determined the diffusion of two relatively monodisperse casein micelle size fractions at $20^{\circ} \mathrm{C}$ using inelastic light scattering. The diffusion for the hydrodynamic radius of micelles, $R_{\mathrm{h}}=76.7 \mathrm{~nm}$ with a concentration of $0.037 \mathrm{~g} / 100 \mathrm{~mL}$ and $216.5 \mathrm{~nm}$ with a concentration of $0.06 \mathrm{~g} / 100 \mathrm{~mL}$ were $2.8 \times 10^{-12} \mathrm{~m}^{2} \mathrm{~s}^{-1}$ and $0.9 \times 10^{-12} \mathrm{~m}^{2} \mathrm{~s}^{-1}$, respectively.

For observation times $>20 \mathrm{~ms}$ and concentrations $>4 \mathrm{wt} \%$ restricted diffusion was measured, with the measured diffusion coefficients decreasing upon increasing the experimental observation times. Within this range of observation times the average intercasein aggregate spacing can be determined. The mean square displacements were calculated to be in the range of $\sim 0.1$ to $0.4 \mu \mathrm{m}$. Unfortunately because of the limited range of $\Delta$ available we were not able to trace this as a function of Na-caseinate concentration.

Ideally, polydispersity inherent within the sample manifests itself as a multiexponential decay in the attenuation data (equation 2.14 and Fig. 3.4). With the distribution of diffusion coefficients correlating to the different casein aggregate sizes being retrieved by performing an inverse Laplace transformation of the attenuation data. In Fig. 3.18 is shown a representative diffusion spectrum obtained from the attenuation data at $\Delta=20 \mathrm{~ms}$ for a 6 $\mathrm{wt} \%$ sample. Analysis of the diffusion coefficients at the limits of the obtained distribution yields a size distribution spanning 0.16 to $0.98 \mu \mathrm{m}$, mapping well the measured size distribution. As discussed above the NMR technique is blind to the smallest aggregates in the dispersion because of the very small contribution they make on a volume basis, compared to 
the number basis for the static light scattering experiments, hence we only detect a portion of the full size distribution. The limits of the distribution obtained via the inverse Laplace transformation carry uncertainties of approximately $50 \%$.

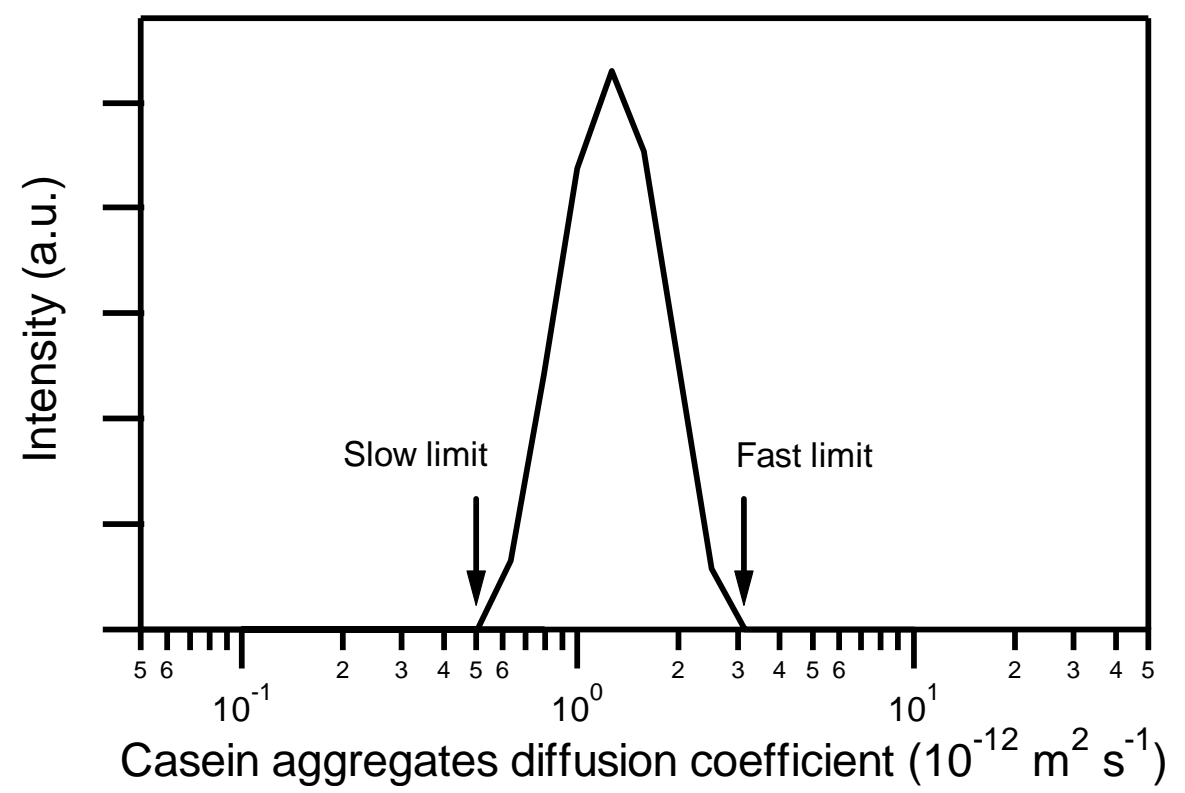

Figure 3.18: Casein aggregate diffusion coefficient distribution for the $6 \mathrm{wt} \% \mathrm{Na}$-caseinate dispersion obtained by performing inverse Laplace transformation on the attenuation data shown in Fig. 3.4. The arrows indicate the diffusion coefficients corresponding to the fast and slow diffusion limits.

\subsubsection{Rheology}

Considering the data presented in Fig. 3.6, there are three domains that have been identified. The first, the initial rise in stress, referred to as the start up. After the start up, the stress is maintained over an extensive time period, before a sharp increase in stress is observed. This response is seen irrespective of applied shear rate or Na-caseinate concentration. Thus it is a fundamental response of these materials. To explain this behaviour we invoke the "Soft Glassy Rheology' (SGR) model or commonly known as the 'trap model', a rather simplistic, generic model to explain the non-linear rheology of soft matter ${ }^{20-27}$.

The start up, suggests the instantaneous jamming and caging of casein aggregates resulting in an immediate increase in the stress response of the material. This is because local elements are trapped in cages formed by their neighbours so that they cannot move ${ }^{23}$.

After the start up, in the plateau region, the small non-linearity in the stress response of the sample, for all imposed shear rates, alludes to a reorganisation of the elements driven for 
example by thermal activation. This may be explained in the context of the Bouchaud et al. trap model, for which it is postulated that an energy landscape of traps with various depths exists. Hence aggregates that are confined within the traps, can become activated and in doing so are able to overcome the energy barrier and "hop" to another trap ${ }^{20,21}$. In this way reorganisation of the traps and cages occurs. This feature is shown in the inset of Fig. 3.6 and can be deduced as a less stable region where the aggregates are in a continual state of break up and renewal.

The long-time behaviour, characterised by the marked increase in the stress response of the material is associated with an irreversible transition to an agglomerated aged state, forming a highly connected three-dimensional gel system. (Note that this gel differs from that formed by the high $\mathrm{Na}$-caseinate concentration dispersions at rest, which is not the result of agglomerations.) The rise in the stress in this region can be thought of as being due to a complex interplay between irreversible ageing and shear-induced effects.

Collectively then, the long-time behaviour of the system is the result of the domination of the delicate interactions between the aggregates while the aggregate's soft sphere-like properties are responsible for the short-time behaviour (i.e., the start up).

Based on the above argument one would predict that as a function of increasing Nacaseinate concentration, gelation should occur at shorter times, due to the already enhanced packing interactions. This is as was observed (Fig. 3.10).

For a given Na-caseinate concentration the response of the material to different applied shear rates, may be explained based on the balance between the energy and the depth of the traps. At low shear rates, the energy given to the system is small such that traps with medium depth are able to trap an 'element'. As the shear rate increases, only those traps of high enough energy and depth will trap an element. As there are fewer deep traps in the energy landscape of traps, more time is needed for the system to fully explore the landscape in order to find the traps capable of trapping the high energy 'elements'. Therefore to achieve the same final stress within the dispersion more time will be required for the system exposed to the higher shear rates. This is reflected in the normalised data of Fig. 3.7 which shows that as the shear rate is increased, the stress plateau exists for longer times before the onset of gelation.

The frequency response of the dispersions on increasing Na-caseinate concentration shown in Fig. 3.15 follows a power law function with an obvious cross over seen in the frequency sweep of the $18 \mathrm{wt} \%$ sample. There are two possible reasons for this observed 
cross over at higher frequencies; (a) the aggregates are more deformed at higher frequency, hence they store more energy; or (b) the interaggregate interaction results in further aggregation/flocculation of the aggregates. Thus, a transition from viscous-dominant to elastic-dominant behaviour occurs when the frequency exceeds the characteristic reorganisation time of the dispersion. This type of transition was also observed in the dynamic time sweep measurements (Fig. 3.16).

Surprisingly, although Na-caseinate is relatively surface active, from Fig. 3.12 and Fig. 3.15 we see that in the linear regime the material does not produce a particularly elastic interface, since the loss modulus $\left(G^{\prime \prime}\right)$ is always larger than the storage modulus $\left(G^{\prime}\right)$. Therefore on a molecular length scale the response of the material is that of a fluid, despite the samples macroscopically not undergoing flow. This is true up to the highest concentrations of Na-caseinate. Hence the water penetrated casein aggregates remain fluid even upon gelation, here we consider both the gelation that occurs on increasing Na-caseinate concentration (i.e., a weak flocculated gel) and that induced by shearing (a highly connected three-dimensional gel) (Fig. 3.12 and Fig. 3.16). Only at the longest times does the response of the system finally change to that of a locally elastic material (Fig. 3.16). This long-time response state is irreversibly achieved and is macroscopically gelled.

\subsection{Conclusions}

For a neutral $\mathrm{pH}$ system, casein aggregate diffusion measurements indicate that interaggregate interactions begin to be important in Na-caseinate dispersions at concentrations as low as $6 \mathrm{wt} \%$ and begin to dominate the system by $10 \mathrm{wt} \%$. Only at the lowest concentrations do the aggregates behave independently. This finding maps with the measured water diffusion data which can be divided into two classes, each of which is modelled separately, with the transition occurring at $\sim 10 \mathrm{wt} \%$ Na-caseinate.

The stress response of Na-caseinate dispersions was examined by subjecting the samples to a constant shear rate as a function of time. At short times, local transient 'caged' or 'jammed states' occur, where the casein aggregates are intermittently rearranged by a simultaneous breaking and forming of the aggregates. At long times, this is followed by a subsequent aged or gelled state where the system is arrested by collective, long-range and aggregate interactions. This transition is irreversible. The higher the Na-caseinate 
concentration and the lower the applied shear rates, the faster this gelled state forms.

A transition from viscous-dominant to elastic-dominant in the dynamic time sweep as demonstrated by a $12 \mathrm{wt} \%$ sample is due to irreversible gelation. Dynamic frequency sweeps for the $16 \mathrm{wt} \%$ sample and below show predominantly liquid-like behaviour. At higher concentration, the system demonstrates a solid-like character, in the sense that $G^{\prime}>G^{\prime \prime}$ over some oscillation frequency. This unexpected observation of a solid-like regime merits further investigation. 


\subsection{References}

1. L. Pepper and H. M. Farrell Jr., J. Dairy Sci. 65, 2259-2260 (1982).

2. P. H. Stothart and D. J. Cebula, J. Mol. Biol. 160, 391-395 (1982).

3. H. M. Farrell Jr., H. Pessen, E. M. Brown and T. F. Kumosinski, J. Dairy Sci. 72, 562-574 (1989).

4. P. H. Stothart, J. Mol. Biol. 208, 635-638 (1989).

5. H. M. Farrell Jr., H. Pessen, E. M. Brown and T. E. Kumosinski, J. Dairy Sci. 73, 3592-3601 (1990).

6. B. Chu, Z. Zhou, G. Wu and H. M. Farrell Jr., J. Colloid Interface Sci. 170, 102-112 (1995).

7. A. HadjSadok, A. Pitkowski, T. Nicolai, L. Benyahia and N. Moulai-Mostefa, Food Hydrocolloids 22, 1460-1466 (2008).

8. L. K. Creamer and G. D. Berry, J. Dairy Res. 42, 169-183 (1975).

9. C. Holt and L. Sawyer, J. Chem. Soc. 89, 2683-2692 (1993).

10. F. Mariette, D. Topgaard, B. Jönsson and O. Söderman, J. Agric. Food Chem. 50, 4295-4302 (2002).

11. K. I. Moughal, P. A. Munro and H. Singh, Int. Dairy J. 10, 683-690 (2000).

12. J. H. Wang, J. Am. Chem. Soc. 76, 4755-4763 (1954).

13. B. Jönsson, H. Wennerström, P. G. Nilsson and P. Linse, Colloid Polym. Sci. 264, 7788 (1986).

14. L. Coppola, C. La Mesa, G. A. Ranieri and M. Terenzi, J. Chem. Phys. 98, 5087-5090 (1993).

15. H. Jóhannesson and B. Halle, J. Chem. Phys. 104, 6807-6817 (1996).

16. P. G. Nilsson and B. Lindman, J. Phys. Chem. 87, 4756-4761 (1983).

17. D. Farrer and A. Lips, Int. Dairy J. 9, 281-286 (1999).

18. K. Venu, V. P. Denisov and B. Halle, J. Am. Chem. Soc. 119, 3122-3134 (1997).

19. C. V. Morr, S. H. C. Lin, R. K. Dewan and V. A. Bloomfield, J. Dairy Sci. 56, 415418 (1973).

20. J. P. Bouchaud, J. Phys. I 2, 1705-1713 (1992).

21. C. Monthus and J. P. Bouchaud, J. Phys. A 29, 3847-3869 (1996). 
22. P. Sollich, F. Lequeux, P. Herbaud and M. E. Cates, Phys. Rev. Lett. 78, 2020-2023 (1997).

23. P. Sollich, Phys. Rev. E 58, 738-759 (1998).

24. S. M. Fielding, P. Sollich and M. E. Cates, J. Rheol. 44, 323-369 (2000).

25. G. A. van Aken and F. D. Zoet, Langmuir 16, 7131-7138 (2000).

26. D. Boon, P. Coussot, H. T. Huynh, G. Bertrand and G. Debrégeas, Europhys. Lett. 59, 786-792 (2002).

27. T. D. Dimitrova and F. Leal-Calderon, Adv. Colloid Interface Sci. 108, 49-61 (2004). 


\section{Chapter 4}

\section{Emulsion Phase Space}

\subsection{Introduction}

The mutual oil-water solubilisation properties of emulsifiers may be summarised in a phase diagram. Phase diagrams are most often used to depict the thermodynamic phase behaviour for a particular mixture as a function of composition, temperature and/or pressure. However, they may also be used to summarise the phase behaviour of kinetically stabilised systems such as emulsions.

Specifically, phase diagrams can be used, for example, to predict and estimate the limits of the separation efficiency (the stability) upon changing oil type. Therefore, fundamental knowledge may be obtained by exploring the correlation between the phase diagrams and the microstructure characteristics according to the chemical nature of the oils and/or emulsifiers. Thus enabling questions such as, for a given composition does the microstructure vary with a change in the chemical nature of the oil, and what is the difference in the stability of the molecular aggregates specific to two different compositions over a certain time scale, to be answered.

In Chapter 3 we reported on the interaction between Na-caseinate aggregates in aqueous phosphate buffer as explored using PGSTE-NMR and rheology techniques for which a number of different self-assembled states were identified. In the literature, the phase behaviour of Na-caseinate mixed with a second biopolymer has also been reported. Among others are the work by Blonk et al. ${ }^{1}$, Capron et $a .^{2}{ }^{2}$ and Simeone et al. ${ }^{3}$ where the phase separation of a mixture of Na-caseinate and Na-alginate in an aqueous solution was determined. In addition to analysis of the separated phases after centrifugation, Blonk et al. also used confocal scanning laser microscopy to quantify the concentration and phase volume of the labelled biopolymers in the mixtures.

Antonov et al. on the other hand used rheo-small angle light scattering, optical 
microscopy, phase analysis, dynamic light scattering, fast protein liquid chromatography, environment scanning electron microscopy and rheology techniques to study the intermacromolecular interactions of this biopolymer mixture with and without the presence of salt in order to understand how this affects its phase equilibrium and morphology ${ }^{4}$. It was found that addition of salt in trace concentrations $\left(10^{-3} \mathrm{wt} \%\right)$ leads to demixing of the biopolymer mixtures and a substantial increase in storage and loss moduli of the system. At high shear rates, Na-caseinate becomes less compatible with Na-alginate in the presence of salt than at rest.

Perrechil et al. ${ }^{5}$ investigated the phase separation of Na-caseinate-locust bean gum gels under acidic conditions using confocal scanning laser microscopy and rheological measurements. A visual phase diagram was constructed at different biopolymer concentrations and four different types of systems, resulting from the different kinetics of phase separation and gel formation were observed: one-phase gels, two-phase gels, one-phase liquid mixtures and two-phase liquid mixtures.

HadjSadok et al. established the phase diagram of the solution mixture as a function of the simultaneous variation of Na-caseinate and xanthan concentrations ${ }^{6}$. Effects of the concentration of the mixture in an aqueous medium on the rheological properties were also investigated. It was deduced that the existence of a critical concentration of Na-caseinate, which depends on the xanthan quantity, and beyond which repulsive segregation interactions occur, generates a weakening of the elastic modulus and an embitterment of the colloidal system structure. The thermodynamic behaviour of the ternary water-caseinate-alginate systems has also been reported ${ }^{2,7-9}$.

Lévy et al. composed a temperature-pressure diagram of oil (peanut oil)-in-water Nacaseinate emulsions by studying the impact of freezing kinetics (fast or low pressure release) on the characteristics of ice crystals and the phase transition of the oil droplets examined under light microscopy ${ }^{10}$. Nonetheless, because of the variability of the chemical compositions and the molecular weights (different sources, batches, etc.) of commercial Nacaseinate and food grade oils, the phase diagrams of emulsions formed using these materials are not universally established. Hence, there remains in the literature a large gap in knowledge with respect to how emulsion microstructure varies with composition.

The data presented in this chapter therefore makes our contribution to filling this gap, providing data which will enable us to answer to above questions, as well as others. Here we report on the macroscopic phase behaviour of emulsions formed at room temperature using 
commercial Na-caseinate as the emulsifier, the oils being the commercial oils; soybean oil and palm oil and a straight-chain hydrocarbon; tetradecane. In subsequent chapters their microstructure-phase properties will be detailed. The phase space was determined at $25^{\circ} \mathrm{C}$. The choice of the different types of oil makes possible the comparison and identification of the phase behaviours and microstructure modifications induced by the interaction between the emulsifier and the oil. Furthermore, emulsions containing Na-caseinate as the emulsifier are of importance in many industrial applications and better information on their solutionbased assemblies is required. The corresponding thermodynamic behaviours for these three systems were also qualitatively determined.

\subsection{Results}

The phase diagrams of the three different systems were constructed for oil concentrations in the range of 50 to $70 \mathrm{wt} \%$ and 1 to $9 \mathrm{wt} \%$ of Na-caseinate. Hundreds of samples were prepared for each system. The phase space of each of the three systems was probed via the determination of the onset of macroscopic phase separation for each sample. Boundaries within the phase space were determined by making samples of closer wt $\%$ differential. Within the stable emulsion phase space, all emulsions present as white macroscopically homogeneous suspensions. Outside of this boundary, samples phase separate instantaneously upon preparation with an excess of oil on the top and a stable emulsion at the bottom. A schematic of this is shown in Fig. 4.1. The stability of the opaque white emulsions could be varied by changing the oil and emulsifier concentrations used.

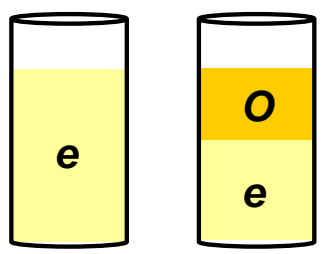

Figure 4.1: Schematic of stable and unstable emulsions formed immediately after homogenisation, within (left) and beyond (right) the stable emulsion phase space boundary in the phase diagram.

The phase diagram detailing the emulsion states for the soybean oil/Na-caseinate/water system is shown in Fig. 4.2. Six distinct emulsion domains can be identified. Note that in general, and as the concentration of Na-caseinate was increased, a greater number of stable emulsions form at lower oil content. 
Domain I: At low Na-caseinate content $(<1.3$ wt\%@ $<60.5$ wt $\%$ soybean oil $)$, the viscosity of the emulsions is low with the fluid flowing easily. All samples destabilise within one day resulting in an upper condensed emulsion and a lower expelled aqueous (here phosphate buffer) phase.

Domain II: At higher Na-caseinate content (domain II: $1.3 \leq c<2.3 \mathrm{wt} \%$ ), emulsions have good stability over a period of $\sim 1$ week, before destabilising.

Domain III: $2.3 \mathrm{wt} \% \leq c<5.5 \mathrm{wt} \% @ 50 \mathrm{wt} \%$ soybean oil, the viscosity of the emulsions is greater than those emulsions in domain I and II, i.e., the fluid does not flow easily. The observed stability is reduced compared to that seen in domain I, and the rate of serum separation at the bottom of the sample vessel is greatly increased. These emulsions are the most unstable.

Domain IV: stabilised over a very narrow range of oil ( $\sim 52.5$ to $55.3 \mathrm{wt} \%)$ and Nacaseinate concentrations ( $\sim 3.2$ to $4.7 \mathrm{wt} \%)$. This domain has similar stability to domain II, but with an enhanced viscosity.

Domain V: Once the Na-caseinate concentration reaches a high value $(\geq 5.5 \mathrm{wt} \% @ 50$ $\mathrm{wt} \%$ soybean oil or for oil concentration $\geq 66 \mathrm{wt} \%$ ), an emulsion droplet network forms which is primarily driven by Na-caseinate self-assembly. This network reorganises slowly, and hence the system is much more stable to serum separation. Emulsions destabilise after $\sim 3$ weeks. It is the most viscous of all the emulsions.

The black dots in the phase diagram represent domains where a transient gel/cream is formed. Here the aggregated oil droplets are trapped in an immobile configuration. The collapse of this state, i.e., serum separation at the bottom of the sample vessel occurs after several months.

The underlying thermodynamic behaviour was also explored for state diagrams corresponding to the red lines shown in Fig. 4.2. These two state diagrams were chosen to correspond to 1) a number of different emulsion microstructures being realised in the kinetic system at a constant oil concentration and 2) a constant protein concentration. In order to probe the thermodynamic state, samples were mixed by hand followed by centrifugation. The cycle was repeated many times over until no further macroscopic change was observed. Samples were monitored over several months. 


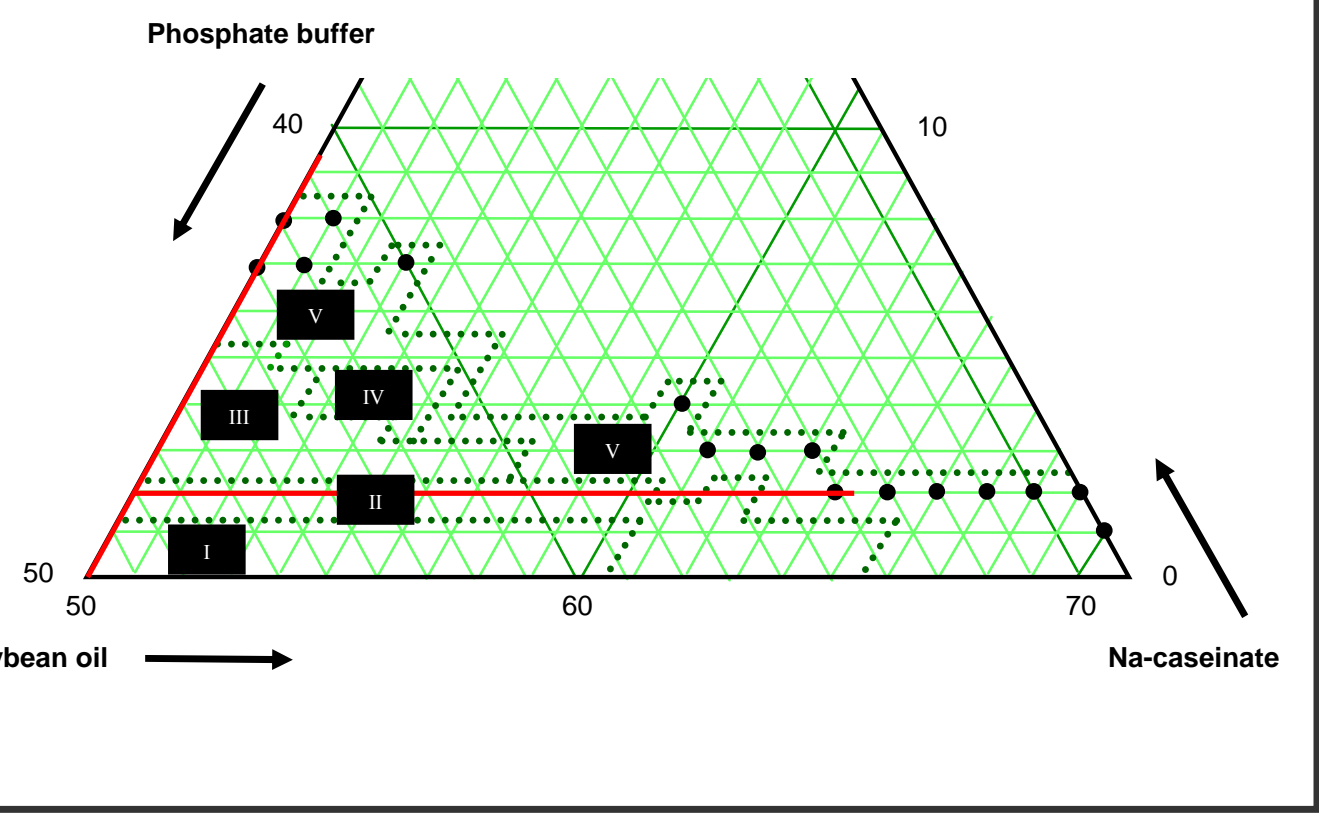

Figure 4.2: Emulsion ternary phase diagram for soybean oil/Na-caseinate/water system at room temperature. The different domains are detailed in the text. The red lines represent the two state diagrams where the corresponding thermodynamic behaviour of the system was studied.

Fig. 4.3 (a) shows a photograph of samples prepared with $50 \mathrm{wt} \%$ soybean oil and Nacaseinate concentrations from 1 to $9 \mathrm{wt} \%$. All samples exhibited as two or three coexisting layers. The volume of the lower aqueous phase remains essentially constant. The top yellowish layer is the oil-rich layer. The $6 \mathrm{wt} \% \mathrm{Na}$-caseinate sample appears as three layers (domain $\mathrm{V}$ in the kinetic phase space). The oil-rich and water-rich layers were separated by the middle layer. This $6 \mathrm{wt} \% \mathrm{Na}$-caseinate sample is believed to be a transition point, separating the two layer samples. The first two layer system consists of an upper mixture of soybean oil, Na-caseinate and water and a lower aqueous region (1 to $5 \mathrm{wt} \%$ Na-caseinate). The second two layer system has an upper soybean oil layer separated from a lower aqueous layer (8 to $9 \mathrm{wt} \%$ Na-caseinate).

In contrast, considering the phase diagram presented in Fig. 4.2, stable emulsions form with $\leq 6 \mathrm{wt} \%$ Na-caseinate while gel formation is observed at higher Na-caseinate concentration. While, the structure of the thermodynamic states was not explored, the bottom (water-rich) layer becomes increasingly viscous and turbid on increasing Na-caseinate concentration. This is in good agreement with the emulsions formed under homogenisation where the viscosity of the samples increases on incrementing Na-caseinate concentration. 
Fig. 4.3 (b) is a photograph of samples prepared at a fixed Na-caseinate concentration, here, $2 \mathrm{wt} \%$. Upon increasing soybean oil concentration (from 50 to $64 \mathrm{wt} \%$ ) the volume of the top layer increases on incrementing the oil concentration. As can be seen in Fig. 4.2, for $2 \mathrm{wt} \% \mathrm{Na}$-caseinate state diagram, we encounter three phase boundaries, i.e., domains II, V and "dots", but the underlying thermodynamic behaviour demonstrated in Fig. 4.3 (b) does not deviate from the trend, two layers samples were observed for all soybean oil concentrations.

(a)

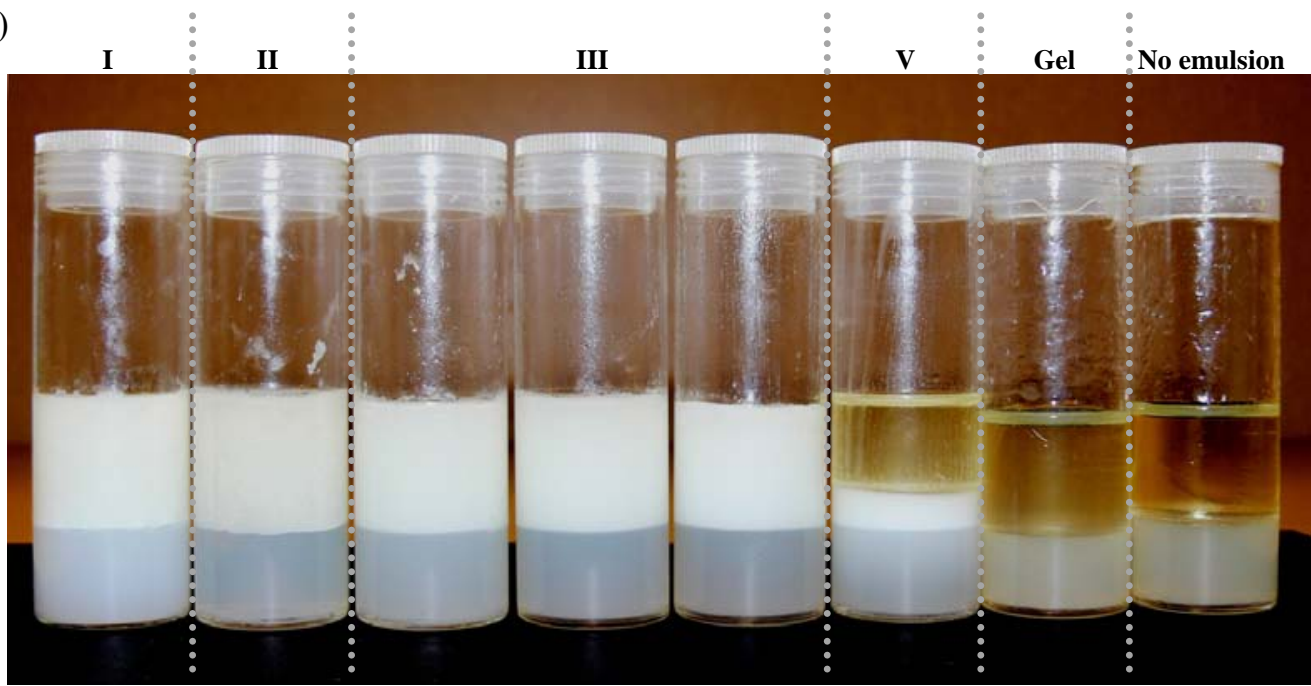

(b)

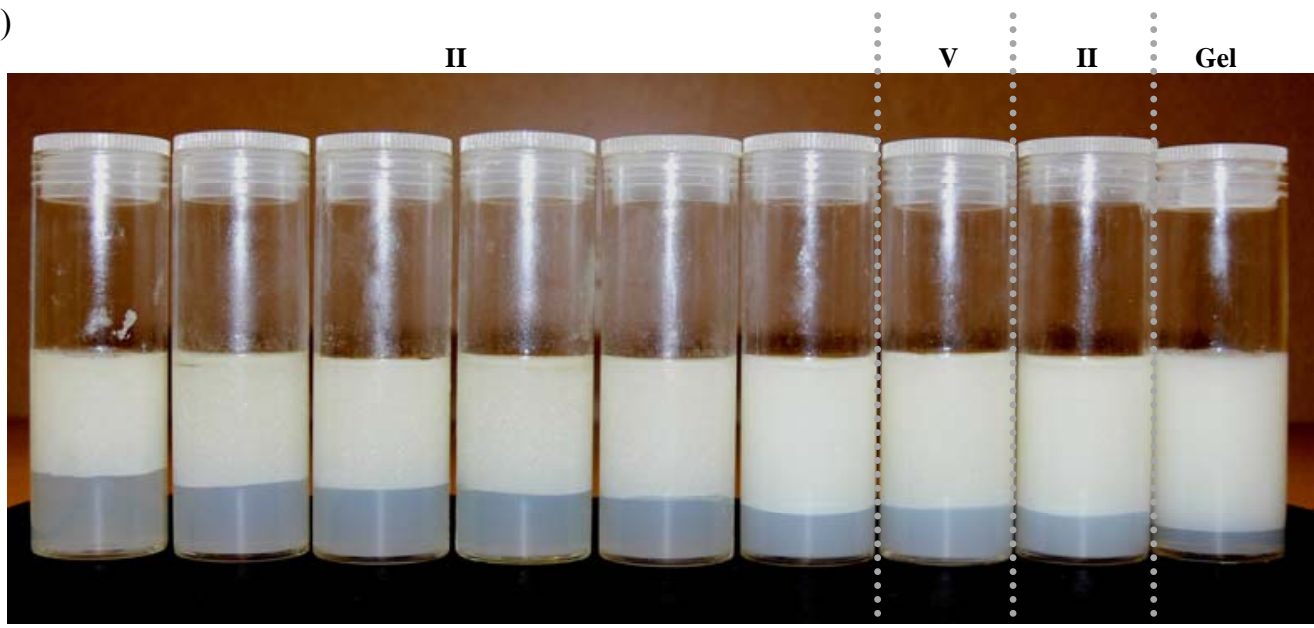

Figure 4.3: Photograph of thermodynamically stabilised samples within the phase space probed prepared with (a) a soybean oil concentration of $50 \mathrm{wt} \%$ and Na-caseinate concentration of 1, 2, 3, 4, 5, 6, 8 and $9 \mathrm{wt} \%$, from left to right and (b) a Na-caseinate concentration of $2 \mathrm{wt} \%$ and soybean oil concentration of 50, 52, 54, 56, 58, $60,61,62$ and $64 \mathrm{wt} \%$, from left to right. The dotted lines show the domain boundaries in the emulsion phase space. 
The emulsion phase diagram for the palm oil/Na-caseinate/water system is shown in Fig. 4.4. The phase diagram is very similar to that of the soybean oil/Na-caseinate/water system. This is expected since both commercial oils are blends and while their exact mix is different there are strong similarities. Stable emulsions can be formed for higher concentrations of Na-caseinate compared to the soybean oil system. Four domains as described in soybean oil system are also present in the palm oil system.

Domain I extends to higher Na-caseinate concentrations ( $\sim \mathrm{wt} \%$ Na-caseinate as compared with $1.3 \mathrm{wt} \%$ in the soybean oil system) but is slightly reduced in its oil extent. The unstable domain III is now reduced in size, extending only up to $\sim 54 \mathrm{wt} \%$ palm oil as compared with $\sim 57 \mathrm{wt} \%$ soybean oil. The range over which domain II extends, as for domain I and III is also more limited with respect to increasing oil concentration as compared with when soybean oil is the discrete phase. Note that domain IV in the soybean oil system is not evident here but domain II encompasses the compositional range occupied by domains IV and $\mathrm{V}$ in the soybean oil system. The most stable domain, still called domain $\mathrm{V}$ to avoid confusion, is now the most extensive domain. As for soybean oil system it is formed at for all oil concentrations. A gel-like paste forms at the periphery of the emulsion phase space.

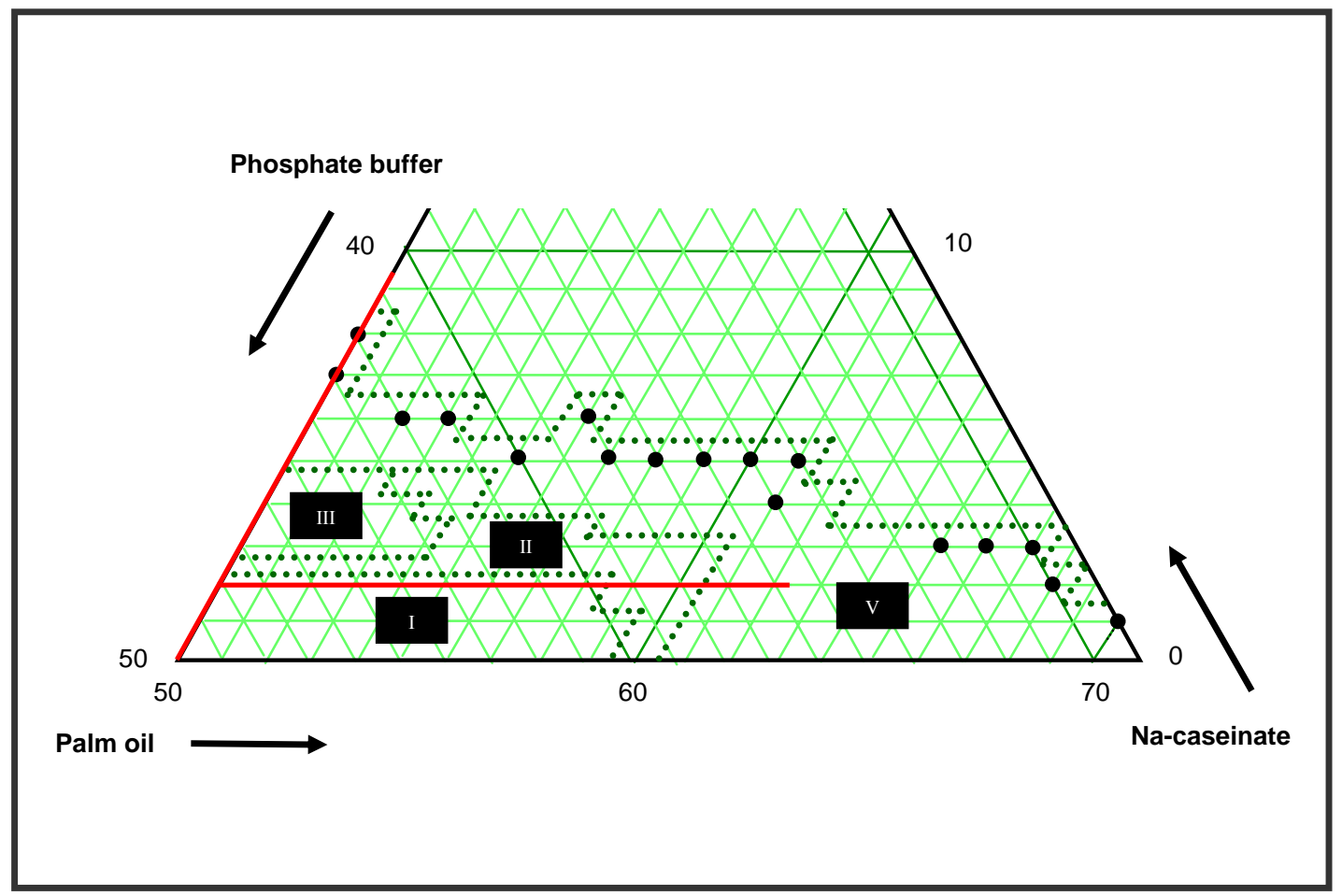

Figure 4.4: Emulsion ternary phase diagram for palm oil/Na-caseinate/water system at room temperature. The different domains are detailed in the text. The red lines represent the two state diagrams where the corresponding thermodynamic behaviour of the system was studied. 
The thermodynamic behaviour of the palm oil system was also investigated in a manner identical to that described for the soybean oil system. Representative samples are shown in Fig. 4.5 showing variation with Na-caseinate and palm oil concentration. All samples exist as two layers despite the observed difference in the degree of macroscopic phase separation depicted in Fig. 4.4. No three layer samples were formed. While the samples shown in Fig. 4.3 for the soybean oil system have an opaque white top layer, the samples shown in Fig. 4.5 for the palm oil system consists of the yellowish oil layer. Moreover, the transition sample containing 6 wt $\%$ Na-caseinate (see Fig. 4.3 (a)) is absent in the palm oil system. The turbidity of the bottom water-rich layer decreases while the viscosity increases with incrementing Na-caseinate and palm oil concentrations. This is particularly obvious in Fig. 4.5 (b) with the volume of the bottom layer decreasing but not as dramatic as that observed in the soybean oil system (Fig. 4.3 (b)).

Finally, the emulsion phase diagram for the tetradecane/Na-caseinate/water system is shown in Fig. 4.6. The tetradecane system required more input energy in order to achieve initial emulsification as compared with the soybean oil and palm oil systems, especially for emulsions prepared above $3 \mathrm{wt} \% \mathrm{Na}$-caseinate and $55 \mathrm{wt} \%$ tetradecane. During initial passthroughs shear-induced flocculation was evident, an excess of tetradecane remained at the surface and a floc structure was clearly visible. Only after 6 to 8 passthroughs was a homogeneous white dispersion obtained. Emulsions cannot be stabilised above $65 \mathrm{wt} \%$ tetradecane as compared with $\sim 70 \mathrm{wt} \%$ for the soybean oil and palm oil systems. On a volume fraction basis this upper limit is more similar in the three systems at around 71 to 73 vol\% (densities of soybean oil, palm oil and tetradecane are 0.850 (measured), 0.866 (measured) and $0.767 \mathrm{~g} \mathrm{~cm}^{-3}$, respectively). The viscosity of tetradecane emulsions is considerably higher than those of the other two systems at the same compositions, irrespective of whether a wt $\%$ or vol $\%$ basis is considered. We note that the Na-caseinate concentration dependence seen for the soybean oil and palm oil systems is no longer observed in the tetradecane system. As can be seen from Fig. 4.6, the domain of the emulsion state is less extended toward the tetradecane/Na-caseinate corner. Only two distinct domains can be identified.

Domain I: Phase separation occurred within a day. The viscosity of the emulsions increases on increasing Na-caseinate and tetradecane concentration. In the most concentrated part of this domain the samples are very viscous, resisting flow. 
(a)

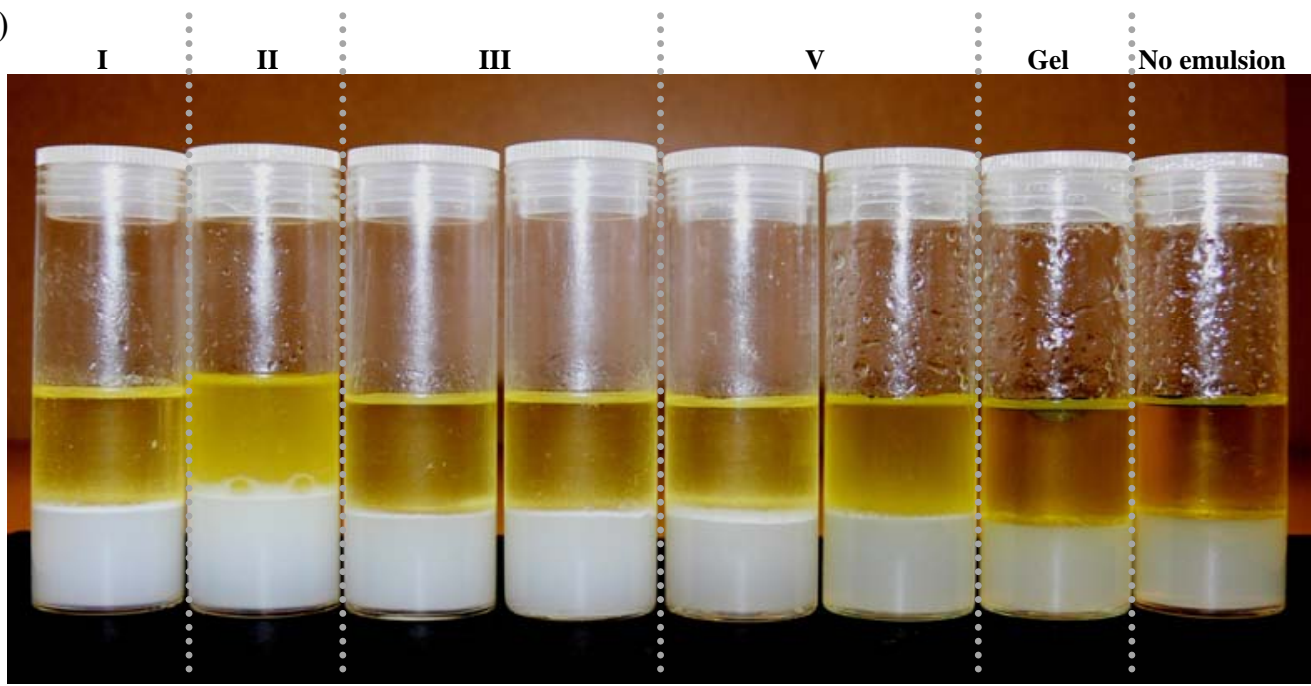

(b)

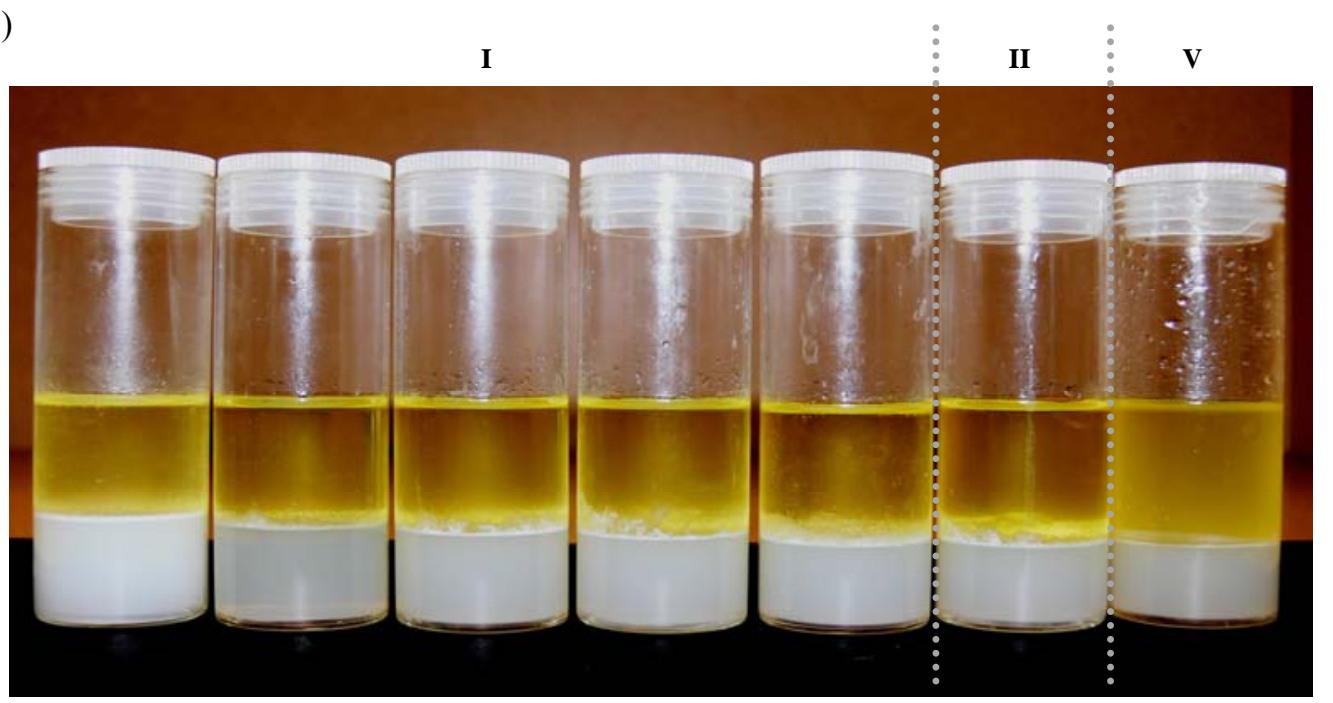

Figure 4.5: Photograph of thermodynamically stabilised samples within the phase space probed prepared with (a) a palm oil concentration of $50 \mathrm{wt} \%$ and Na-caseinate concentration of 1, 2.5, 3, 4, 5, 6, 8 and $9 \mathrm{wt} \%$, from left to right and (b) a Na-caseinate concentration of $2 \mathrm{wt} \%$ and palm oil concentration of 50, 52, 54, 56, 58, 60 and $62 \mathrm{wt} \%$, from left to right. The dotted lines show the domain boundaries in the emulsion phase space.

Domain II: The phase separation process is increasingly retarded; emulsions destabilise after $\sim 1$ week. This domain extends to much higher Na-caseinate and tetradecane concentrations than in the soybean oil and palm oil systems. However, the most stable domain, i.e., domain $\mathrm{V}$, is not formed here.

As with the other two systems, gel/cream formation (represented by the black dots) produces an aggregated droplet network where the emulsions are stable for several months. 


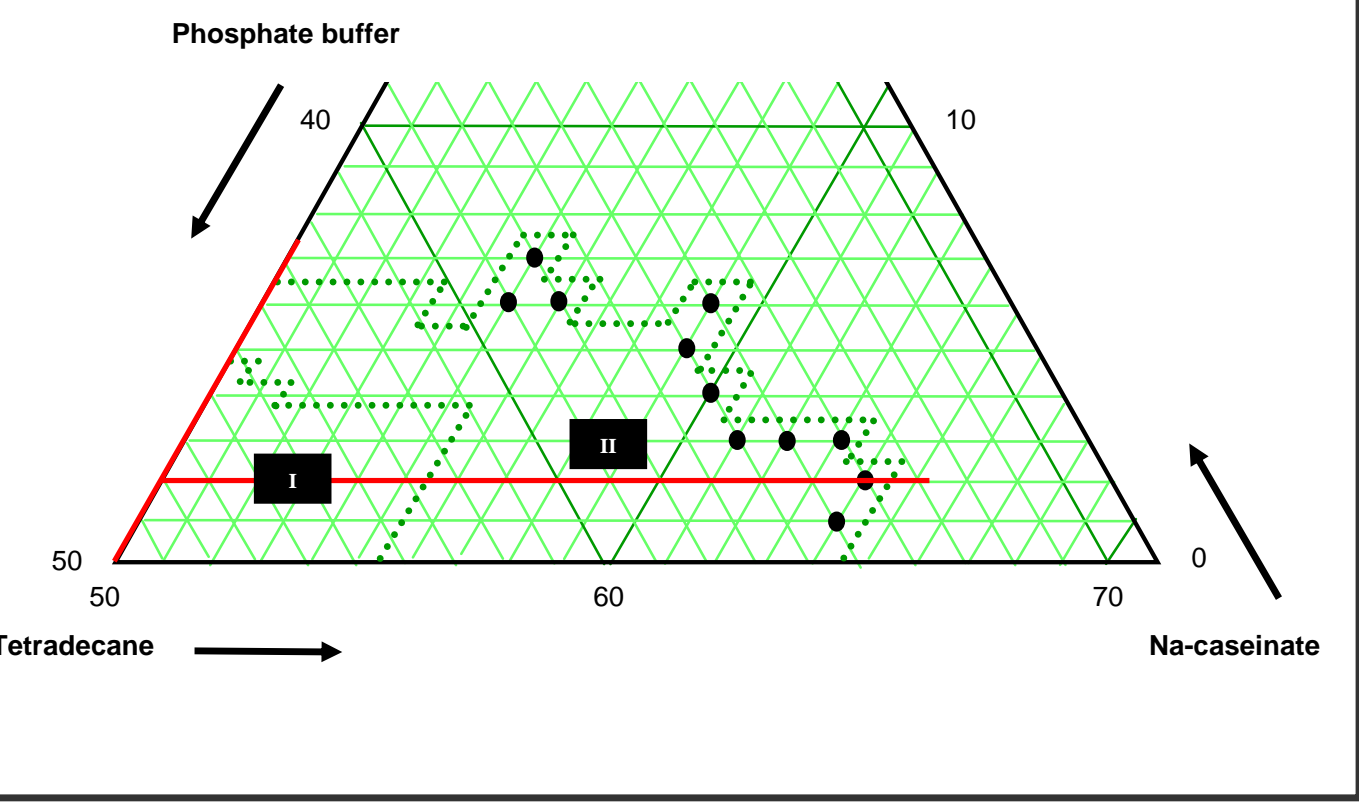

Figure 4.6: Emulsion ternary phase diagram for tetradecane/Na-caseinate/water system at room temperature. The different domains are detailed in the text. The red lines represent the two state diagrams where the corresponding thermodynamic behaviour of the system was studied.

The thermodynamic behaviour of the tetradecane/Na-caseinate/water system is exemplified by the samples shown in Fig. 4.7. Samples show the formation of two or three layers reminiscent of the soybean oil system despite the emulsion phase diagrams being so different. The top transparent layer is oil-rich. The $6 \mathrm{wt} \%$ sample $(50 \mathrm{wt} \%$ tetradecane, Fig. 4.7 (a)) appears as three phases. The middle layer is turbid. These two samples which separate the two layer samples consisting of the opaque white top layer from the clear tetradecane layer correspond to the underlying thermodynamic behaviour of the emulsions formed in domain II of the phase diagram in Fig. 4.6.

As shown in Fig. 4.7 (b), increasing tetradecane concentration; and thereby decreasing water concentration, leads to an increase and decrease of the volume of the top and bottom layers, respectively. The effect of increasing tetradecane concentration on the emulsion phase behaviour at the state diagram containing $2 \mathrm{wt} \%$ Na-caseinate shown in Fig. 4.6 does not yield any variation in the corresponding thermodynamic behaviour (Fig. 4.7 (b)). This is similar to that observed in the soybean oil and palm oil systems. 
(a)

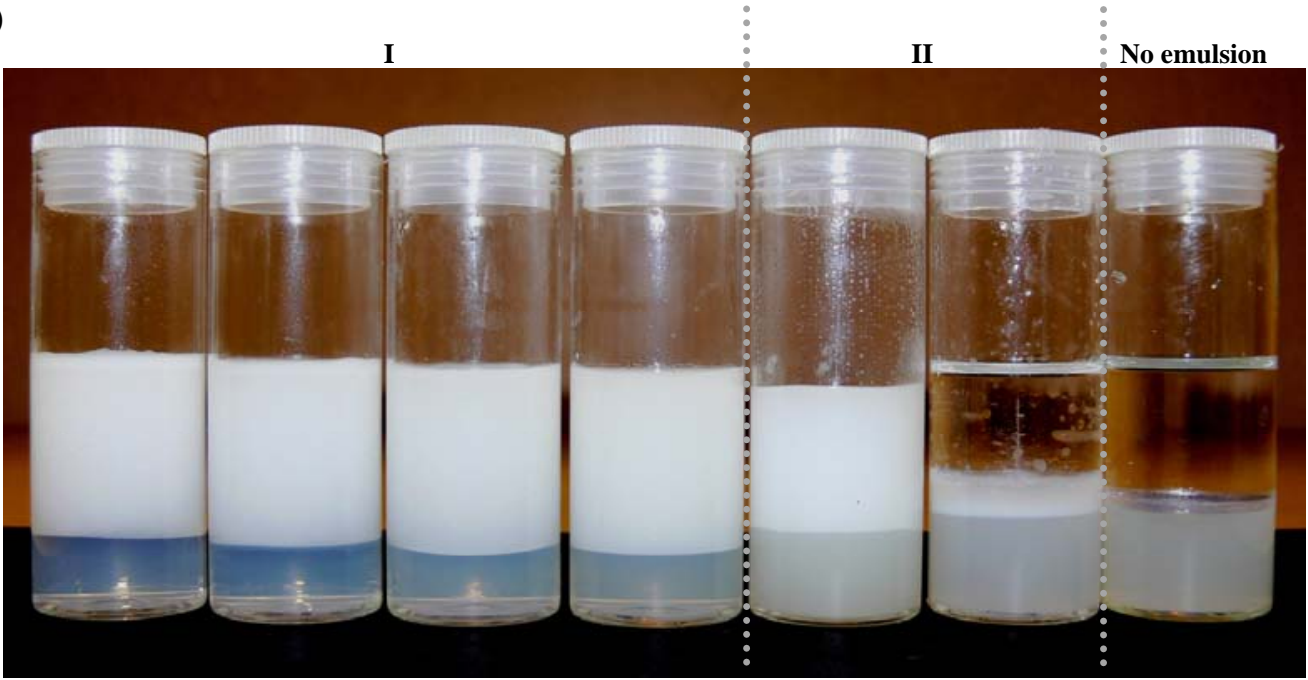

(b)

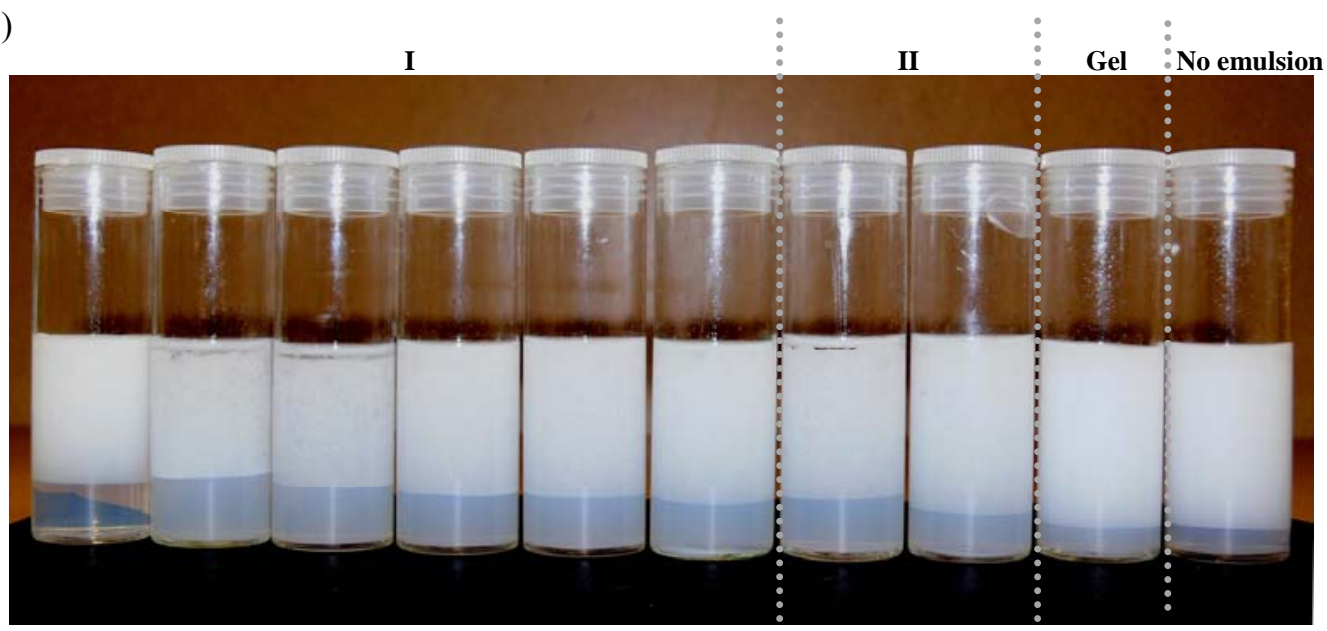

Figure 4.7: Photograph of thermodynamically stabilised samples within the phase space probed prepared with (a) a tetradecane concentration of $50 \mathrm{wt} \%$ and Na-caseinate concentration of 1, 2, 3, 4, 5, 6 and $7 \mathrm{wt} \%$, from left to right and (b) a Na-caseinate concentration of $2 \mathrm{wt} \%$ and tetradecane concentration of 50, 51, 52, 53, 54, 55, $56,60,64$ and $65 \mathrm{wt} \%$, from left to right. The dotted lines show the domain boundaries in the emulsion phase space.

Comparing the three phase diagrams, two points can be noticed:

(i) gel-like pastes that remain stable for months are formed near the demixing line, denoted by dots in all three of the emulsion phase diagrams occurring for Na-caseinate concentrations of $\sim 3 \mathrm{wt} \%$ for soybean oil and $\sim 5$ to $6 \mathrm{wt} \%$ for palm oil and tetradecane.

(ii) the main difference between the phase diagrams composed of food grade triglycerides (soybean oil and palm oil) and a laboratory-used straight-chain hydrocarbon (tetradecane) is the existence of two (domains II and III of palm oil system) or three (domains II, III and IV of soybean oil system) liquid-liquid isotropic regions in the phase diagrams of 
food grade oil systems which separate a less stable region (domain I) from a very stable region (domain $\mathrm{V})$.

\subsection{Discussion}

The efficiency of commercial Na-caseinate in stabilising and destabilising oil-in-water emulsions can be widely found in the literature ${ }^{11-13}$. Although $\kappa$-casein is believed to be able to protect the surface of newly formed oil droplets in caseinate-based emulsions against recoalescence via steric stabilisation ${ }^{14,15}$, the interaction potential between adsorbed casein layers determines the ultimate colloidal stability of casein-coated emulsion droplets ${ }^{16}$. If at all values of surface-to-surface separation $D$ the net interaction energy $A(D)$ is repulsive, the emulsion will be stable. But, if the interaction energy is substantially attractive over some range of separation, then the oil droplets will be destabilised. In practice, the occurrence of destabilisation depends on the overall balance of electrostatic forces, steric forces, and van der Waals forces ${ }^{17}$. The thickness/structure of the adsorbed casein layers define the combined steric and electrostatic contributions to $A(D)$.

What follows is a discussion on the destabilisation process ${ }^{18}$ of emulsions that must be considered when considering emulsion stability and behaviour.

Coalescence ${ }^{19-22}$, i.e., fusion of oil droplets through rupture of the film separating the oil droplets and reversible or irreversible flocculation due to collisions (see Table 4.1 for types of collision) between oil droplets resulting in discrete aggregates or a network structure ${ }^{23}$ with no change in the droplet size or droplet size distribution are the two most common mechanisms of instability ${ }^{24}$. These processes may occur simultaneously. Each of them strongly depends on droplet size and on key parameters such as interfacial properties (essential for coalescence) and the extent of the repulsive barrier (important for flocculation).

The pressure differential across the interface drives coalescence of the droplets. Here, when two droplets encounter each other drainage of the thin film formed between them ${ }^{25-28}$ occurs resulting in the formation of a single larger droplet. Depending on the thickness and viscosity of the film, the geometry of the droplets the coalescence rate varies in different systems. 
Table 4.1: Various types of collision in emulsions and their corresponding flocculation rate ${ }^{29}$

\begin{tabular}{lll}
\hline$d N / d t$ & $d r / d t$ & $r(t)$ \\
\hline $\begin{array}{l}\text { Brownian flocculation }{ }^{30} \\
\frac{d N}{d t}=-\frac{4}{3} \frac{k_{B} T}{\eta} N^{2} \frac{1}{w_{B}}\end{array}$ & $\frac{d r}{d t}=\frac{2}{3} \frac{k_{B} T}{\eta} \frac{\phi}{\pi r^{2}} \frac{1}{w_{B}}$ & $r=\left(r_{0}+\frac{\phi 2 k_{B} T}{9 \pi \eta w_{B}}\right)^{1 / 3}$ \\
Shear-induced flocculation: laminar flow ${ }^{31}$ & $\frac{d r}{d t}=\frac{4 \phi r S}{3 \pi} \frac{1}{w_{S}}$ & $r=r_{0} \exp \left(\frac{4 \phi S t}{3 \pi} \cdot \frac{1}{w_{S}}\right)$ \\
$\frac{d N}{d t}=-\frac{16}{3} N^{2} S r^{3} \frac{1}{w_{S}}$ & $\frac{d r}{d t}=\frac{3 \beta}{2} \sqrt{\frac{\varepsilon_{\text {int }}}{\eta}} \phi r \frac{1}{w_{T}}$ & $r=r_{0} \exp \left(\frac{3 \beta}{2} \sqrt{\frac{\varepsilon_{\text {int }}}{\eta} \phi t \cdot \frac{1}{w_{T}}}\right)$ \\
Shear-induced flocculation: turbulent flow ${ }^{32}$ & $\frac{d N}{d t}=-6 \pi \beta \sqrt{\frac{\varepsilon_{\text {int }}}{\eta} r^{3} N^{2} \frac{1}{w_{r}}}$ & \\
\hline
\end{tabular}

Note: The rate of flocculation is expressed in terms of change in number concentration $(d N / d t)$, the increase in particle radius $(d r / d t)$ or as the particle radius as a function of time.

$N=$ particle concentration; $t=$ time; $k_{B}=$ Boltzmann constant; $T=$ absolute temperature; $\eta=$ viscosity; $\phi=$ volume fraction dispersed phase; $w_{B}=$ stability factor for Brownian flocculation according to Fuchs ${ }^{33} ; w_{S}=$ stability factor for shear-induced flocculation; $w_{T}=$ stability factor for flocculation in turbulent flow; $\beta=\mathrm{a}$ constant describing the relation between the diffusive flux in a turbulent flow field and the liquid velocity.

Creaming and flocculation are the two destabilisation terms that are closely associated with each other. Creaming is often the mechanism driving destabilisation in emulsions that leads to the observed serum separation ${ }^{34}$. Creaming happens when there is a density difference between the dispersed and continuous phases. During creaming the droplet size distribution does not change. The degree of stability of emulsions can be determined by the creaming rate, i.e., by measuring the height of the creaming or serum layer ${ }^{35}$, the droplet size or droplet size distribution. The presence of a large aggregate network of low density droplets increases the creaming rate. The creaming rate, given by Barnea and Mizrahi ${ }^{36}$, is proportional to the square of the oil droplet radius ${ }^{37}$ and the density difference between the dispersed and continuous phase ${ }^{38}$ which is:

$$
\begin{gathered}
v=\frac{v_{s}(1-\phi)}{\left(1+\phi^{\frac{1}{3}}\right)} \exp \left[\frac{5 \phi}{3(1-\phi)}\right], \\
v_{s}=\frac{2 r^{2}\left(\rho_{0}-\rho\right) g}{9 \eta_{0}} \text { and } \\
r^{2}=\frac{\sum_{i} n_{i} r_{i}^{5}}{\sum_{i} n_{i} r_{i}^{3}}
\end{gathered}
$$


where $v_{s}$ is the creaming velocity of an isolated, spherical droplet with radius $r, \rho_{0}$ and $\rho$ are the density of continuous and dispersed phases, respectively, $g$ is the acceleration due to gravity and $\phi$ is the volume fraction of the droplets. Thus, a decrease in both the oil droplet size and density difference between the dispersed and continuous phases means a more stable emulsion results.

Comparison of the oil droplet size is not possible given the inhomogeneity and high polydispersity of soybean oil, palm oil/Na-caseinate/water systems (see cryo-SEM micrographs in Chapter 5 and Chapter 7, respectively) and the freezing difficulty for tetradecane/Na-caseinate/water system (see cryo-SEM micrographs in Chapter 8). While comparison of the density difference between the three systems is too reductionist, the macroscopic separation results presented in the phase diagrams focusing on 50 to $55 \mathrm{wt} \%$ oil $/ 2.25 \mathrm{wt} \% \mathrm{Na}$-caseinate/water state diagram shows that the tetradecane/Na-caseinate/water system is the least stable system, as expected.

Depending on the interaction between the droplets, creaming and the associated state of flocculation are both particularly sensitive to the amount of Na-caseinate used ${ }^{12}$. While protein load at the oil droplet surface was not measured and is not the focus of this thesis, it has been reported that bridging flocculation typically occurs at low protein/oil ratios where there is insufficient protein available to give (near-) saturation of the oil droplet surface $(\Gamma=$ $\left.3 \mathrm{mg} \mathrm{m}^{-2}\right)^{14,39-43}$. This value of protein load on the oil droplet surface is in reasonably good agreement with that found by Dickinson et al. ${ }^{12}$, despite differences in oil type, oil volume fraction and average droplet size. This is also consistent with the situation in our case, i.e., for soybean oil, palm oil/Na-caseinate/water emulsions in domain I. When the oil droplets surface coverage is low, there is a gradual loss of steric/electrostatic repulsion. A net attractive interaction energy between Na-caseinate adsorbed layers on different oil droplets therefore develops resulting in a tendency of the adsorbed protein to be shared between the adjacent oil droplets. Subsequently, the trapped continuous phase within the aggregated structures may be released and the stability of the emulsions is low ( $<1$ day).

For soybean oil, palm oil/Na-caseinate/water emulsions in domain II, the system is thought to have much greater than half-saturation oil droplet surface coverage ${ }^{12}$. The oil droplets are covered by a thick steric stabilisation layer and the unbound protein concentration in the continuous phase is fairly low. Hence, these systems are found to be consistent with a substantial enhancement of stability where no discernible change in mean droplet size over the storage period of $\sim 1$ week. 
In the case of domain III in soybean oil, palm oil/Na-caseinate/water systems, serum separation was observed within a day. We attribute this to a different mechanism of flocculation, because at these protein concentrations the oil droplets surfaces have near optimal coverage and with an excess of unbound emulsifier molecules or aggregates in the continuous phase $\mathrm{s}^{11,12,40,44-48}$. Flocculation is believed to follow a depletion process ${ }^{49,50}$ as depicted in Fig. 4.8. Theory has shown ${ }^{51,52}$ that depletion flocculation may also be induced by small particles as well as polymers. In protein-stabilised emulsions, as two neighbouring oil droplets approach each other to a distance less than the mean diameter of a non-adsorbing Na-caseinate aggregate ${ }^{53,54}$, the aggregate is effectively excluded from the intervening space $^{55,56}$. Hence, the resulting osmotic pressure gradient creates an attractive interaction between the oil droplets ${ }^{57}$. The system can record a phase separation ${ }^{58}$. The oil droplet ${ }^{59}$ and aggregate sizes, as well as the concentration of aggregates in solution determine the strength of the flocculation. For the interaction to have a substantial effect on emulsion stability, $\left|\Delta G_{\text {dep }}\right|$, the magnitude of the energy change associated with the depletion effect ${ }^{60,61}$, must be well in excess of $k T$, otherwise floc structures will tend to break up under the influence of thermal motion ${ }^{62}$.

(a)

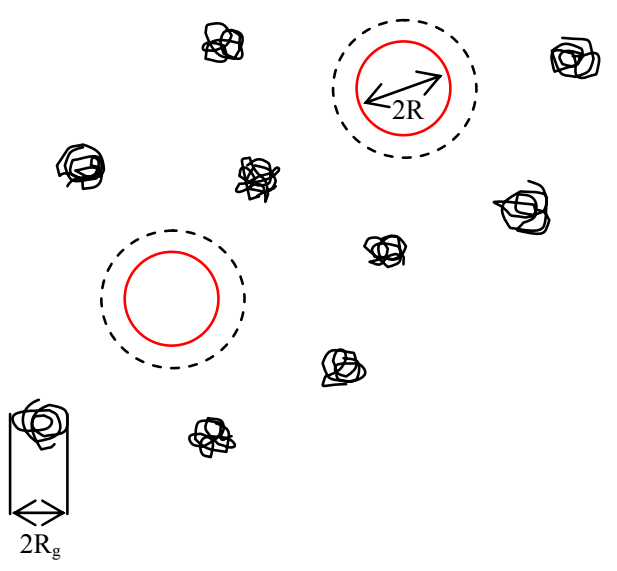

(b)

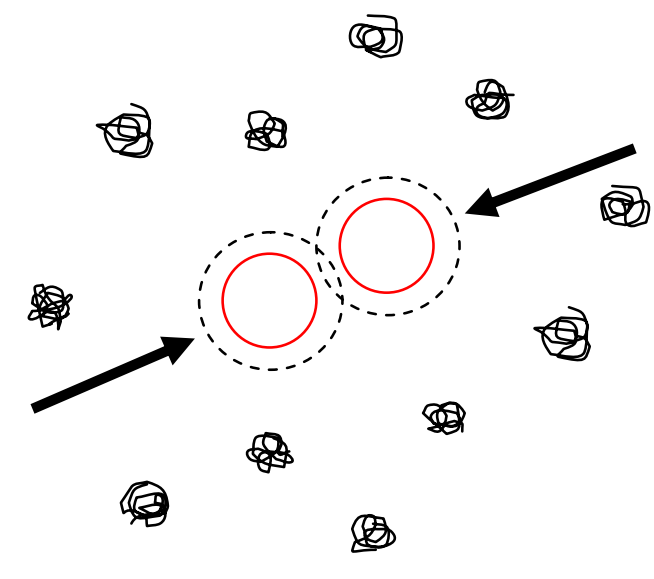

Figure 4.8: Schematic picture of two spherical colloid particles of radius $\mathrm{R}$ in a solution containing nonadsorbing proteins of radius of gyration $\mathrm{R}_{\mathrm{g}}$, with protein coils excluded from a depletion zone (dashed line) near the particle surface: (a) no overlap of depletion layers (osmotic pressure on the sphere is isotropic); (b) overlapping depletion layers (net attractive force between particles from unbalanced osmotic pressure). The excess osmotic pressure is indicated by the arrows ${ }^{63}$. 
Emulsions in domain $\mathrm{V}$ for soybean oil, palm oil/Na-caseinate/water systems exhibit "initial" serum separation after $\sim 3$ weeks. It is anticipated that, at such a sufficiently high $\mathrm{Na}$-caseinate concentration, the formation of flocculated structures are more extensive with an increasing number of unbound Na-caseinate molecules and aggregates in the aqueous phase. Indeed, in addition to the higher oil volume fraction concentrating the protein, it has been shown previously in Chapter 3 that Na-caseinate self-assembles to form a threedimensional network in pure phosphate buffer solution at protein concentration as low as 6 $\mathrm{wt} \%$. As such, it is probable that in domain $\mathrm{V}$ protein self-assembly induces the formation of an extended network resulting in more stable emulsions being formed.

During extended storage, evidence has been put forward that a locally more closepacked particle gel network may develope ${ }^{64,65}$ permeating the emulsion, which stabilises the system against so-called buoyancy forces through rapid consolidation and restricted floc movement ${ }^{13,38,66-68}$. The strength of the interparticle attractive forces will eventually become strong enough to inhibit rearrangement.

On switching to tetradecane, a water insoluble straight-chain hydrocarbon, the emulsifier/oil interactions dominate the system, the complexity of the phase space is reduced and the ability to form a highly stable emulsion in which the Na-caseinate induces the formation of a three-dimensional network within the aqueous continuum is removed.

Gel formation, a phenomenon ubiquitous in nature, was recently interpreted as an arrested phase separation (in the short-range attractive colloidal system) where the tendency of the system to form an inhomogeneous structure is frozen by the tendency of the particles to stick together ${ }^{69,70}$. It is this interconnected flocculated droplet network which is the determining factor in relation to the susceptibility of the emulsion to separation under gravity $^{71}$.

In line with understanding the particle dynamics in a gel network of emulsion systems, Watson and co-workers carried out a Brownian dynamics simulation for oil droplets of radius, $R=1 \mu \mathrm{m}$ (see 72 for details). Fig. 4.9 is a schematic sketch of the corresponding internal energy per particle, $E$, relative to the thermal background (left ordinate) and the mean square displacement relative to the free particle diffusion (right ordinate) as a function of time from Brownian dynamics trajectories. 


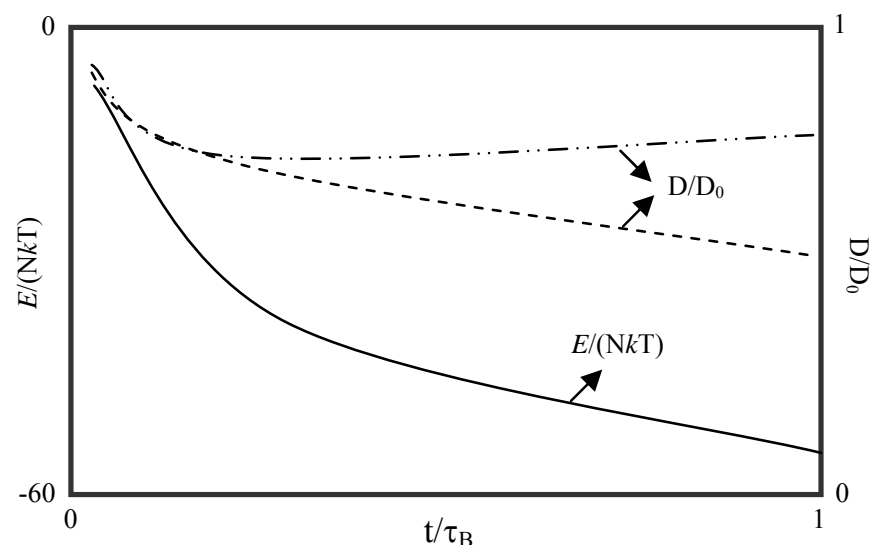

Figure 4.9: For a purely repulsive system, the internal energy of each particle (not shown) is static on the scale of the graph while the mean square displacement approaches equilibrium after over one Brownian time unit ( - - ) ). After 'switching on' the attractive forces, there is a sharp decrease in the internal energy $(-\cdots)$ and the mean square displacement (-) as the particles are now confined in aggregated clusters.

The emulsion phase diagrams for the soybean oil, palm oil/Na-caseinate/water systems are generally more similar to each other than to the emulsion phase diagram of the tetradecane/Na-caseinate/water system. Mapping the underlying thermodynamic behaviour of the soybean oil system shown in Fig. 4.3 (a) with the corresponding kinetic phase space (Fig. 4.2), it can be noticed that emulsions in domains I to III have the same base thermodynamic behaviour. A three-phase system exists for samples in domain $\mathrm{V}(6 \mathrm{wt} \% \mathrm{Na}-$ caseinate) which is bordered by domain III and gel formation; with an emulsion being stabilised in the middle layer between the oil and the water phases. On further increasing Nacaseinate concentration at fixed and low oil concentration, gel formation dominates the system leading to an inability to form emulsions. In fact, enhanced interaggregate interaction forming a gelled state was observed in Na-caseinate dispersions at Na-caseinate concentration as low as $6 \mathrm{wt} \%$ (see Chapter 3). However, in Fig. 4.3 (b) we see that for samples in domain V (63 wt\% soybean oil) bordered by domain II and gel formation a twoinstead of three-phase system exists. This is in contrast with what is observed in Fig. 4.3 (a) indicating that true gel formation in the kinetic phase space is defined by the Na-caseinate concentration and not the oil concentration and the two gel states obtained at the high Nacaseinate concentration at low oil content and at the low Na-caseinate concentration at high oil content in the phase space are different. The earlier is initiated by the inherent nature of the aggregation of Na-caseinate but the latter is caused by the enhanced interdroplet interaction. 
The subtle differences in the chemical compositions between soybean oil and palm oil manifest themselves as major perturbations to the thermodynamic phase behaviour. Palm oil has a higher percentage of saturated chains than soybean oil increasing its melting point and decreasing its solubility in water. Hence, as can be seen in Fig. 4.5 only two-phase systems are observed with expelled oil existing as the top layer.

For the tetradecane system, the underlying thermodynamic behaviour is almost identical to that for soybean oil system despite the emulsion phase space being completely different. At a fixed Na-caseinate concentration on increasing tetradecane concentration, all samples exist as two-phase (Fig. 4.7 (b)). A three-phase system is observed in Fig. 4.7 (a) for samples in domain II ( $6 \mathrm{wt} \% \mathrm{Na}$-caseinate) with an emulsion existing as the middle layer between the oil and the water phases. Two-phase system, i.e., oil as the top and Na-caseinate dispersion as the bottom layer was formed on further increasing Na-caseinate concentration.

The phase behaviour of the soybean oil and palm oil emulsions is predominantly controlled by the self-assembly of the Na-caseinate. Through the oil volume fraction the concentration of the Na-caseinate in solution is effectively modified and as such those microstructures formed at high total $\mathrm{Na}$-caseinate concentration at low oil content are induced to form at much lower Na-caseinate concentrations on increasing oil concentration. The inherent subtle differences in the chemical compositions of the two commercial oils manifest themselves as minor perturbations to the phase behaviour, changing the interfacial domain since a greater extent of surface coverage is required in the palm oil system compared with the soybean oil system. This is evident in domain I which extends to higher protein concentrations resulting in domain II being reduced in its extent. With a greater amount of protein residing at the oil/water interface boundaries shift to lower oil concentrations and emulsions are stabilised to higher Na-caseinate concentrations $(\sim 3 \mathrm{wt} \%$ compared with $\sim 2$ $\mathrm{wt} \%$ at $70 \mathrm{wt} \%$ palm oil and soybean oil, respectively).

Our findings on the phase behaviour of the tetradecane/Na-caseinate/water system deviate from that reported by Dickinson et al., although they investigated the influence of Nacaseinate concentration on the stability of relatively dilute oil-in-water emulsions (35 or 45 vol $\%$ oil $)^{12}$. Their results indicating that the emulsions formed have a complex dependence on Na-caseinate content with a phase space very similar to that found in the soybean oil, palm oil/Na-caseinate/water systems.

Upon switching from the highly branched unsaturated triglycerides to the straight-chain hydrocarbon; tetradecane the interactions between the oil and the casein adsorbed layers are 
markedly altered. Tetradecane has poor solvent properties compared to soybean oil and palm oil. It is not soluble in water. Hence more energy input is required to produce an emulsion. Oil penetration of tetradecane is expected to be less effective which results in an increase in the bending elasticity ${ }^{73,74}$, leading to a thinner interfacial layer ${ }^{75,76}$. Excess energy input causes shear-induced flocculation during homogenisation as the balance between Gaussian curvature, interfacial tension and Gibbs elasticity is very delicate. Such dramatic changes are mirrored in the phase behaviour of the tetradecane/Na-caseinate/water system, where only two stable domains are observed and the most stable domain (domain V) is not formed.

It should be noted that the two domains in the tetradecane system cannot be fully reconciled with those in the soybean oil and palm oil systems. Bridging and depletion flocculation with respect to the amount of Na-caseinate present in the emulsions prepared cannot be identified. This will be addressed further in Chapter 8 .

\subsection{Conclusions}

Coarse one-phase emulsions are formed for all three systems investigated. The phase diagrams for soybean oil, palm oil/Na-caseinate/water systems are rich and very similar. At low Na-caseinate concentration emulsions bridging flocculation is evident and emulsions are of low stability, increasing protein concentration leads to an increased stability and the existence of distinct individual oil droplets. Further increases reduce emulsion stability due to depletion flocculation. We believed that protein self-assembly is then initiated. At sufficiently high protein and/or oil concentrations this results in the continuous phase being a three-dimensional protein network and emulsion stability is enhanced. At the limits of the emulsion phase space a gel-like paste is formed. Introduction of tetradecane dramatically restricts the diversity of emulsions formed. Protein self-assembly is limited and there is no evidence for network formation. The tetradecane/Na-caseinate/water system has a less extended phase diagram and is found to be energy input sensitive due to the reduction in the extent of penetration of tetradecane to the interface and hence the less rigid oil/water interface. Enhanced flocculation tends to occur at high concentration of Na-caseinate and/or oil. 


\subsection{References}

1. J. C. G. Blonk, J. van Eendenburg, M. M. G. Koning, P. C. M. Weisenborn and C. Winkel, Carbohydrate Polym. 28, 287-295 (1996).

2. I. Capron, S. Costeux and M. Djabourov, Rheol. Acta 40, 441-456 (2001).

3. M. Simeone, A. Alfani and S. Guido, Food Hydrocolloids 18, 463-470 (2004).

4. Y. A. Antonov and P. Moldenaers, Biomacromolecules 10, 3235-3245 (2009).

5. F. A. Perrechil, A. L. M. Braga and R. L. Cunha, Food Hydrocolloids 23, 2085-2093 (2009).

6. A. HadjSadok, N. Moulai-Mostefa and M. Rebiha, Int. J. Food Properties 13, 369-380 (2010).

7. Y. A. Antonov, P. Van Puyvelde and P. Moldenaers, Int. J. Biol. Macromol. 34, 29-35 (2004).

8. Y. A. Antonov, V. Y. Grinberg and V. B. Tolstoguzov, Stärke 27, 424-431 (1975).

9. Y. A. Antonov, V. Y. Grinberg, N. A. Zhuravskaya and V. B. J. Tolstoguzov, J. Texture Stud. 11, 199-215 (1980).

10. J. Lévy, E. Dumay, E. Kolodziejczyk and J. C. Cheftel, Lebensm.-Wiss. u.-Technol. 32, 396-405 (1999).

11. E. Dickinson and M. Golding, Food Hydrocolloids 11, 13-18 (1997).

12. E. Dickinson, M. Golding and M. J. W. Povey, J. Colloid Interface Sci. 185, 515-529 (1997).

13. E. Dickinson and M. Golding, J. Colloid Interface Sci. 191, 166-176 (1997).

14. E. Dickinson, J. Chem. Soc., Faraday Trans. 88, 2973-2983 (1992).

15. E. Dickinson, J. Dairy Res. 56, 471-477 (1989).

16. E. Dickinson, Int. Dairy J. 9, 305-312 (1999).

17. E. Dickinson and G. Stainsby, Colloids in food. (Applied Science, London, 1982).

18. P. Becher, Emulsions: theory and practice. (Oxford University Press, New York, 2001).

19. G. A. van Aken and T. van Vliet, Langmuir 18, 7364-7370 (2002).

20. G. A. van Aken, Langmuir 18, 2549-2556 (2002).

21. G. W. J. Lee and T. F. Tadros, Colloids Surfs. 5, 129-135 (1982).

22. G. W. J. Lee and T. F. Tadros, Colloids Surfs. 5, 117-127 (1982). 
23. V. M. Starov and V. G. Zhdanov, J. Colloid Interface Sci. 258, 404-414 (2003).

24. B. Bergenståhl and G. Östberg, in Emulsions and emulsion stability, edited by J. Sjöblom (Marcel Dekker, Inc., New York, 1996).

25. T. D. Dimitrova, F. Leal-Calderon, T. D. Gurkov and B. Campbell, Langmuir 17, 8069-8077 (2001).

26. G. A. van Aken and F. D. Zoet, Langmuir 16, 7131-7138 (2000).

27. E. Dickinson, B. S. Murray and G. Stainsby, J. Chem. Soc., Faraday Trans. 84, 871883 (1988).

28. E. Nakache, P.-Y. Longaive and S. Aiello, Colloids Surfs. A 96, 69-76 (1995).

29. G. Östberg, B. Bergenståhl and M. Hulden, J. Coatings Technol. 66, 37-46 (1994).

30. M. von Smoluchowski, Z. Phys. 17, 593-604 (1916).

31. M. von Smoluchowski, Z. Phys. Chem. 92, 129-168 (1917).

32. V. G. Levich, Physicochemical hydrodynamics. (Prentice-Hall, Englewood Cliffs, NJ, 1962).

33. N. Fuchs, Z. Phys. 89, 736-742 (1934).

34. E. Dickinson, in Food hydrocolloids: structures, properties and functions, edited by K. Nishinari and E. Doi (Plenum, New York, 1993), pp. 387.

35. E.-H. Liu, P. T. Callaghan and K. M. McGrath, Langmuir 19, 7249-7258 (2003).

36. E. Barnea and J. Mizrahi, Chem. Eng. J. 5, 171-189 (1973).

37. B. P. Binks, Modern aspect of emulsion science. (The Royal Society of Chemistry, UK, 1998).

38. V. J. Pinfield, E. Dickinson and M. J. W. Povey, J. Colloid Interface Sci. 186, 80-86 (1997).

39. E. Dickinson, E. W. Robson and G. Stainsby, J. Chem. Soc., Faraday Trans. 79, $2937-$ 2952 (1983).

40. E. Dickinson, J. A. Hunt and D. G. Dalgleish, Food Hydrocolloids 5, 403-414 (1988).

41. A. R. Mackie, J. Mingins and A. N. North, J. Chem. Soc., Faraday Trans. 87, 30433049 (1991).

42. Y. Fang and D. G. Dalgleish, J. Colloid Interface Sci. 156, 329-334 (1993).

43. D. G. Dalgleish, in Food macromolecules and colloids, edited by E. Dickinson and D. Lorient (Royal Society of Chemistry, Cambridge, 1995), pp. 23.

44. C. Ma, Colloids Surfs. 28, 1-7 (1987).

45. M. P. Aronson, Langmuir 5, 494-501 (1989). 
46. J. Bibette, D. Roux and F. Nallet, Phys. Rev. Lett. 65, 2470-2473 (1990).

47. F. Leal-Calderon, B. Gerhardi, A. Espert, F. Brossard, V. Alard, J. F. Tranchant, T. Stora and J. Bibette, Langmuir 12, 872-874 (1996).

48. E. Dickinson, in Food colloids: proteins, lipids and polysaccharides, edited by E. Dickinson and D. Lorient (Royal Society of Chemistry Cambridge, 1997), pp. 107.

49. S. Asakura and F. Oosawa, J. Chem. Phys. 22, 1255-1256 (1954).

50. D. H. Napper, Polymeric stabilisation of colloidal dispersions. (Academic Press, London, 1983).

51. J. Y. Walz and J. Sharma, J. Colloid Interface Sci. 168, 485-496 (1994).

52. Y. Mao, M. E. Cates and H. N. W. Lekkerkerker, Phys. A 222, 10-24 (1995).

53. J. Bibette, D. Roux and B. Pouligny, J. Phys. II 2, 401-424 (1992).

54. M. P. Aronson, in Emulsions-a fundamental and practical approach, edited by J. Sjöblom (Kluwer, Dordrecht, 1992), pp. 75.

55. E. Dickinson and D. J. McClements, Advances in food colloids. (Blackie Academic and Professional, Glasgow, 1995).

56. X. Ye, T. Narayanan, P. Tong, J. S. Huang, M. Y. Lin, B. L. Carvalho and L. J. Fetters, Phys. Rev. E 54, 6500-6510 (1996).

57. P. Sperry, J. Colloid Interface Sci. 87, 375-384 (1982).

58. A. P. Gast, C. K. Hall and W. B. Russel, J. Colloid Interface Sci. 96, 251-267 (1983).

59. X. L. Chu, A. D. Nikolov and D. T. Wasan, Langmuir 12, 5004-5010 (1996).

60. D. J. McClements, Colloids Surfs. A 90, 25-35 (1994).

61. H. Luyten, M. Jonkman, W. Kloek and T. van Vliet, in Food colloids and polymers: stability and mechanical properties, edited by E. Dickinson and P. Walstra (Royal Society of Chemistry, Cambridge, 1993), pp. 224.

62. E. Dickinson and M. Golding, Colloids Surfs. A 144, 167-177 (1998).

63. R. Tuinier, J. Rieger and C. G. de Kruif, Adv. Colloid Interface Sci. 103, 1-31 (2003).

64. E. Dickinson, An introduction to food hydrocolloids. (Oxford University Press, Oxford, 1992).

65. T. van Vliet and P. Walstra, in Food colloids, edited by R. D. Bee, P. Richmond and J. Mingins (Royal Society of Chemistry, Cambridge, 1989), pp. 206.

66. Y. Cao, E. Dickinson and D. J. Wedlock, Food Hydrocolloids 4, 185-195 (1990).

67. E. Dickinson, Colloids Surfs. A 75, 195-201 (1993). 
68. E. Dickinson, M. I. Goller and D. J. Wedlock, J. Colloid Interface Sci. 172, 192-202 (1995).

69. S. Manley, H. M. Wyss, K. Miyazaki, J. C. Conrad, V. Trappe, L. J. Kaufman, D. R. Reichman and D. A. Weitz, Phys. Rev. Lett. 95, 238302-238305 (2005).

70. G. Foffi, C. D. Michele, F. Sciortino and P. Tartaglia, Phys. Rev. Lett. 94, 078301078304 (2005).

71. D. J. McClements, Food emulsions: principles, practice, and techniques. (CRC Press, Boca Raton, 2004).

72. E. Dickinson, Food Colloids. (The Royal Society of Chemistry UK, 2005).

73. L. T. Lee, D. Langevin, J. Meunier, K. Wonk and B. Cabane, Prog. Colloid Polym. Sci. 81, 209-214 (1990).

74. M. Gradzielski, D. Langevin and B. Farago, Phys. Rev. E 53, 3900-3919 (1996).

75. B. P. Binks, H. Kellay and J. Meunier, Europhys. Lett. 16, 53-58 (1991).

76. S. Chaieb, B. P. Binks and J. Meunier, J. Phys. II 5, 1289-1296 (1995). 


\section{Chapter 5}

\section{Soybean OilEmulsions}

\subsection{Introduction}

Emulsions are widely encountered in food systems. While speculatively it is believed that the microstructure and stability of food emulsions are strongly dependent on the constituents composing these colloids, the manner in which this is achieved is not known. This lack of specific knowledge therefore significantly restricts our ability to control, manipulate and predict emulsion product characteristics. As such it is both crucial and interesting to investigate the relationship between emulsion composition and properties through variation of the types of emulsifiers and oils used in the formulation.

In gaining some insights on the microstructure and stability of food emulsions and in particular those containing a mixture of fats (or oil soluble components, e.g., fatty acids or sterols) and different surface active components (e.g., proteins, phospholipids), we attempt in this chapter to understand the phase behaviour of the emulsifier at the oil/water interface. Moreover, the interactions of the adsorbed layer in association with the oil droplets in concentrated emulsions (dispersed phase $\sim 50$ to $70 \mathrm{wt} \%$ ) are explored. Here, Na-caseinate was used as the emulsifier and commercial soybean oil as the dispersed phase. The fundamental study of these emulsions is important both because of their industrial relevance and the theoretical challenges they present given the vastness of use of food grade oil in caseinate-based emulsions.

\section{$5.2 \quad$ Results}

The phase diagram for this system was discussed in Chapter 4. Five distinct microstructures are formed within the phase space. All are categorised as oil-in-water emulsions. In the 
following, results predominantly for emulsions in domain I to IV will be presented. This is due to the very high viscosity of emulsions in domain $\mathrm{V}$ which was incompatible with most of the characterisation techniques used.

\subsubsection{Laser Diffraction Particle Sizing}

The oil droplet diameter of separately prepared emulsions of the same concentration has an estimated deviation of $\pm 15 \%$ based on repeated measurements of these emulsions. In Fig. 5.1 are shown the droplet size distributions of freshly prepared $50 \mathrm{wt} \%$ soybean oil/1, 2, 3 and 4 $\mathrm{wt} \% \mathrm{Na}$-caseinate/water emulsions. The lognormal form of the distribution for all samples is very similar. The distribution is bimodal and is little affected upon changing Na-caseinate concentrations despite three different domains (domains I to III) being encountered across the phase diagram (see Fig. 4.2).

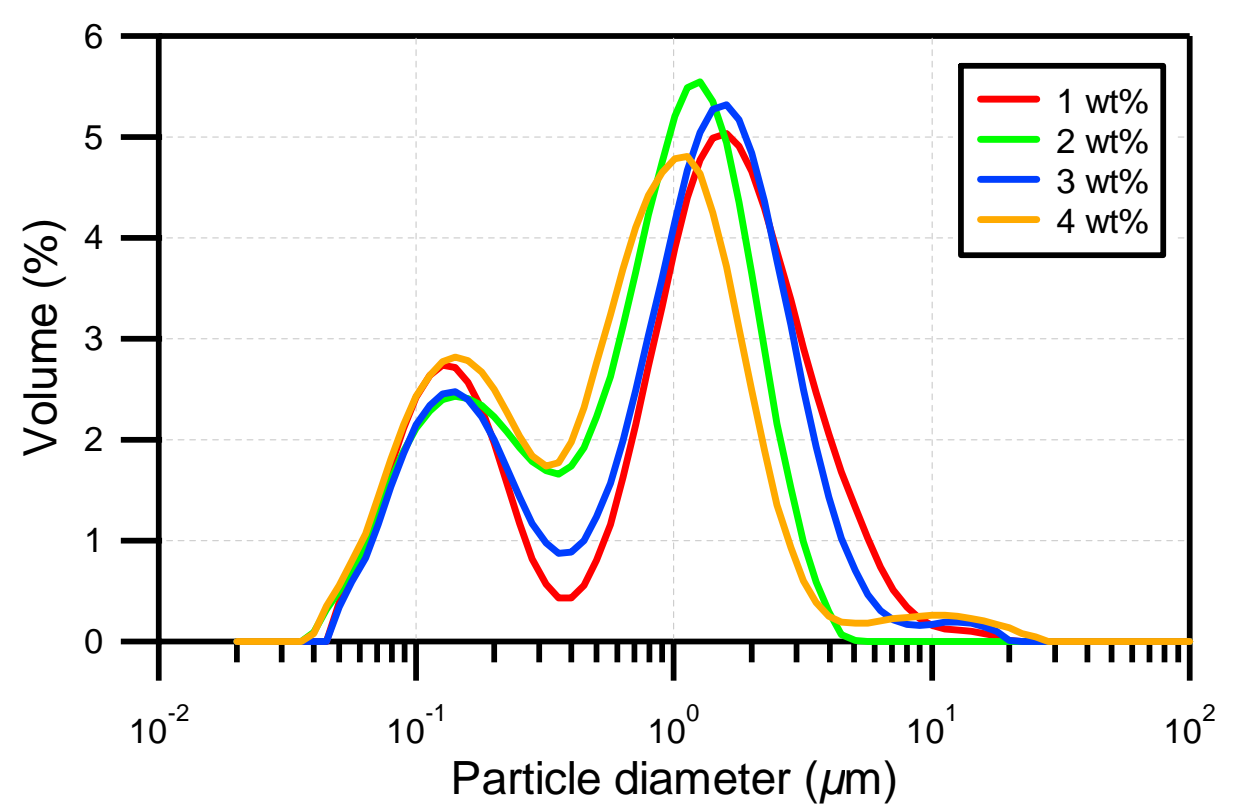

Figure 5.1: Droplet size distributions for freshly prepared $50 \mathrm{wt} \%$ soybean oil/Na-caseinate/water emulsions with 1, 2, 3 and 4 wt $\%$ Na-caseinate.

Oil droplets from $\sim 0.04$ (the limit of the instrument) to $10 \mu \mathrm{m}$ in diameter exist with $D[3,2]$ and $D[4,3]$ values ranging from 0.26 to $0.33 \mu \mathrm{m}$ and from 0.89 to $1.49 \mu \mathrm{m}$, respectively. The lower and higher droplet mode diameters of the bimodal distribution are $\sim 0.13 \mu \mathrm{m}$ and $\sim 1.7 \mu \mathrm{m}$, respectively. The lower droplet mode consists of a mixture of small oil droplets and casein aggregates (see Chapter 3 ) which are believed to directly participate in 
emulsion stability. The third droplet mode observed at $\sim 15 \mu \mathrm{m}$ is most likely due to the existence of a few larger oil droplets and trapped air bubbles in the sample. Such air bubbles are very difficult to remove.

The droplet size distributions of fresh emulsions consisting of a fixed emulsifier concentration but increasing oil concentration, i.e., 50, 52, 54, 56 and $58 \mathrm{wt} \%$ soybean oil/2 wt $\%$ Na-caseinate/water (domain II in the phase diagram) are shown in Fig. 5.2. Similar droplet size distributions were obtained for all samples for a given Na-caseinate concentration with a slight broadening on increasing soybean oil concentration. For a given oil concentration, the distribution is essentially bimodal. The emulsions have oil droplets spanning from $\sim 0.04$ to $10 \mu \mathrm{m}$ in diameter with $D[3,2]$ and $D[4,3]$ values ranging from 0.29 to $0.67 \mu \mathrm{m}$ and from 0.90 to $1.99 \mu \mathrm{m}$, respectively. The lower and higher droplet mode diameters of the bimodal distribution are $\sim 0.15 \mu \mathrm{m}$ and $\sim 1.5 \mu \mathrm{m}$, respectively. Again, trapped air bubbles and a few larger oil droplets manifest themselves as a small, broad peak centred around $15 \mu \mathrm{m}$ for emulsions prepared with 52, 54, 56 and $58 \mathrm{wt} \%$ soybean oil.

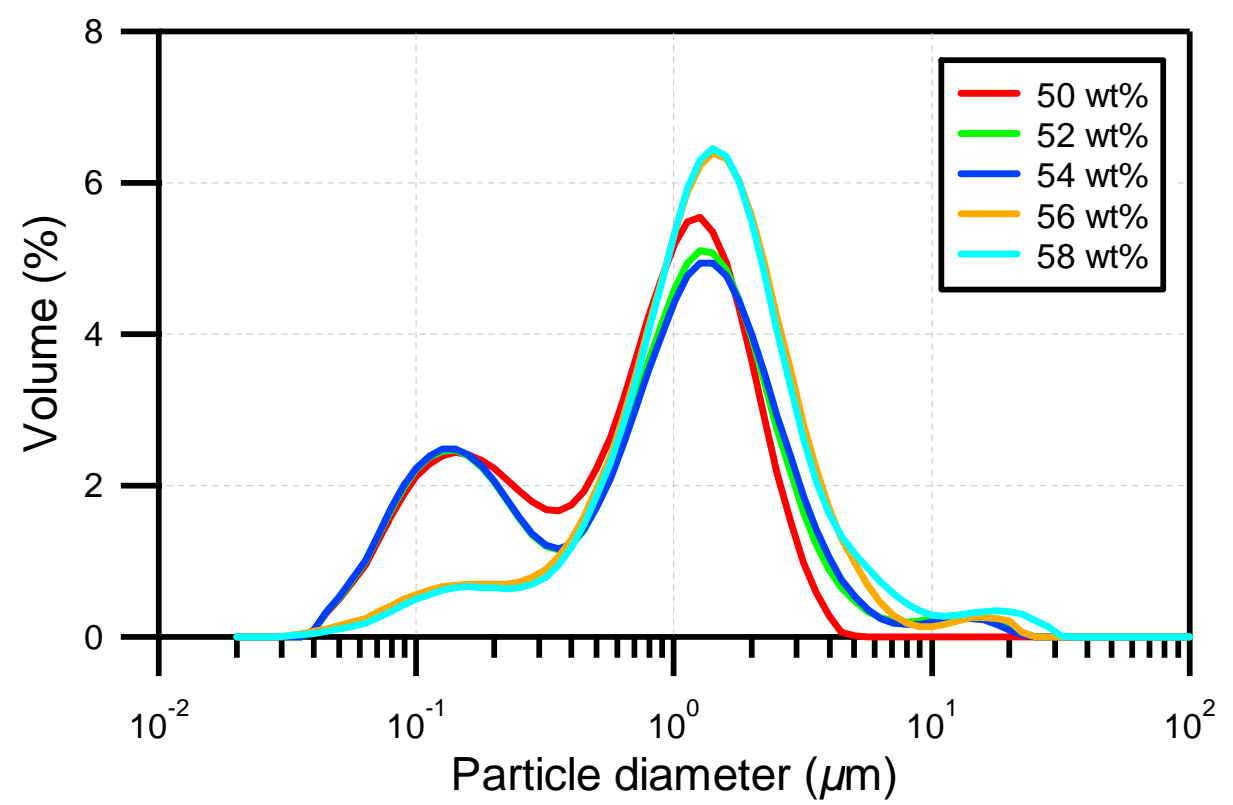

Figure 5.2: Droplet size distributions for freshly prepared soybean oil $/ 2 \mathrm{wt} \% \mathrm{Na}$-caseinate/water emulsions with $50,52,54,56$ and $58 \mathrm{wt} \%$ soybean oil.

The $50 \mathrm{wt} \%$ soybean oil $/ 2 \mathrm{wt} \%$ Na-caseinate $/ 48 \mathrm{wt} \%$ water emulsion will be used to demonstrate destabilisation process in our emulsions. We note that the phase separation of this sample, which is in domain II of the phase diagram, occurred after $\sim 1$ week (see Fig. 4.2). Emulsions in domain II are used as the exemplar system because they have the most 
experimentally accessible time constants for the destabilisation process. Other emulsions within the phase space are not suitable for gathering substantial data for mapping the changes occurring to the sample over a wide time frame. Samples in domain I, for example, phase separate too quickly and samples in domain $\mathrm{V}$ are stable for too long.

The droplet size distribution of the chosen system was observed as a function of time. The results are shown in Fig. 5.3. For the sake of clarity, only the droplet size distributions at three sampling times are presented. Although separation into an oil-rich phase (top), which remains opaque, and a water-rich phase (bottom) was visually detected after $\sim 1$ week, the droplet size distribution only showed significant changes after $\sim 3$ weeks. Initially the droplet mode diameters increase, with the distribution narrowing only from 52 to 68 days. This narrowing is consistent with destabilisation via Ostwald ripening. With time, there is a progression towards the presence of a narrower distribution with $D[3,2]$ and $D[4,3]$ for the $\sim 70$ days old sample being $1.55 \mu \mathrm{m}$ and $3.15 \mu \mathrm{m}$, respectively. The lower droplet mode values remain nearly constant for $\sim 2$ months, while the higher droplet mode value begins to increase after $\sim 3$ weeks. After $\sim 70$ days, the lower droplet mode value had increased from $0.29 \mu \mathrm{m}$ to $0.95 \mu \mathrm{m}$. Over the same time, the higher droplet mode value increased from 1.3 $\mu \mathrm{m}$ to $4.2 \mu \mathrm{m}$. The final distribution spans less than two orders of magnitude, $0.34 \mu \mathrm{m}$ to $13.39 \mu \mathrm{m}$, compared with the approximately two orders of magnitude for fresh emulsions, i.e., a slight narrowing.

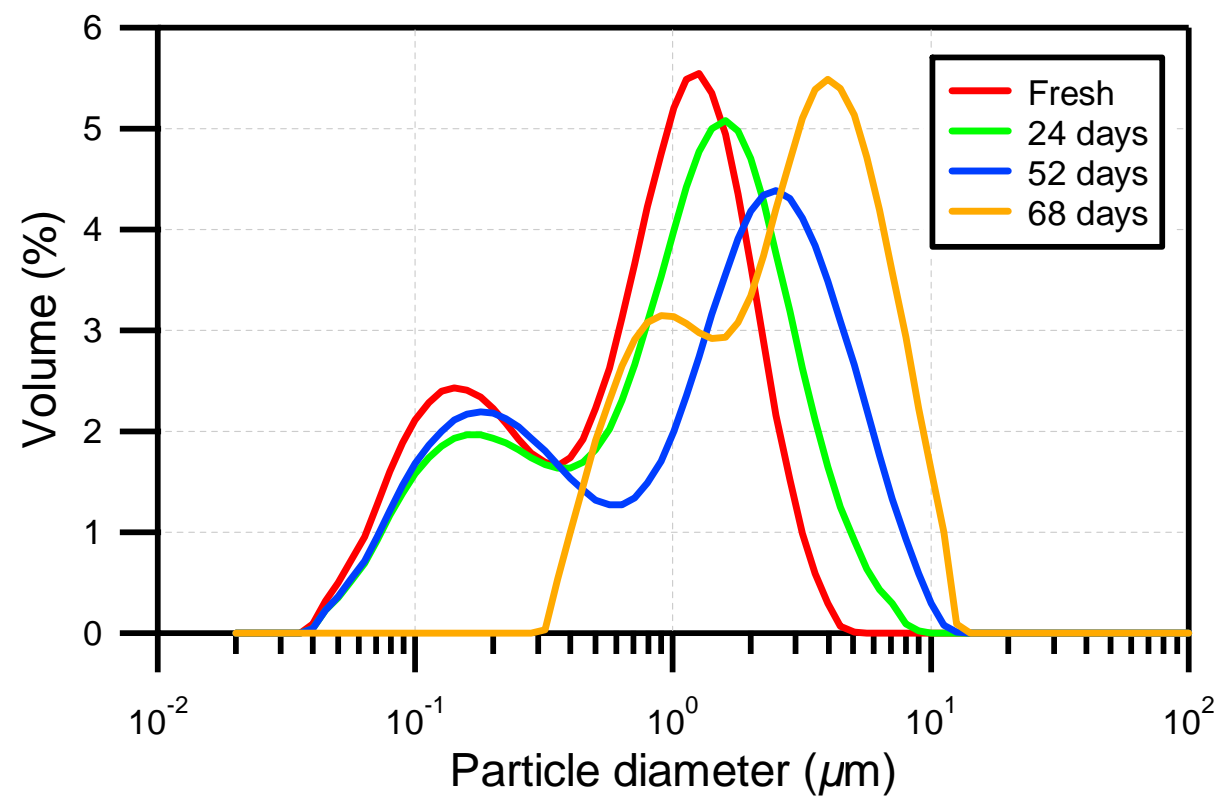

Figure 5.3: Droplet size distributions as a function of time for freshly prepared $50 \mathrm{wt} \%$ soybean oil/2 wt $\% \mathrm{Na}-$ caseinate $/ 48 \mathrm{wt} \%$ water emulsion at sampling time $=24,52$ and 68 days. 


\subsubsection{PGSTE-NMR}

For the emulsions investigated here, the proton signals for Na-caseinate are too weak to be detected and also overlap with those of soybean oil. Hence, only the oil and water domains were studied.

In Fig. 5.4 is shown a characteristic set of single exponential decay attenuation data for water diffusion, here for a $50 \mathrm{wt} \%$ soybean oil $/ 2 \mathrm{wt} \% \mathrm{Na}$-caseinate $/ 48 \mathrm{wt} \%$ water emulsion. The diffusion coefficient of water was measured as a function of the soybean oil concentration using an observation time, $\Delta$ of $20 \mathrm{~ms}$. The data obtained are shown in Fig. 5.5. Due to the high field gradient stability of our PGSTE-NMR equipment, uncertainties are estimated to be $\sim 2 \%$. It is obvious from the data shown in Fig. 5.5 that the water diffusion coefficient decreases steadily on increasing soybean oil concentration. In the case of $1 \mathrm{wt} \%$ $\mathrm{Na}$-caseinate (domain I), the water diffusion coefficient decreases from 1.37 to $1.18 \times 10^{-9}$ $\mathrm{m}^{2} \mathrm{~s}^{-1}$ while in the case of $3 \mathrm{wt} \%$ Na-caseinate (domain III) the change is from 1.03 to $0.91 \times$ $10^{-9} \mathrm{~m}^{2} \mathrm{~s}^{-1}$.

Irrespective of Na-caseinate concentration, upon increasing the soybean oil concentration, the number of oil droplets formed increases and the distance between the oil droplets decreases. The increasing oil droplet interaction is mirrored by the reduction in water diffusion. Moreover, we see that on incrementing Na-caseinate concentration water diffusion is increasingly affected. For $1 \mathrm{wt} \%$ and $2 \mathrm{wt} \%$ Na-caseinate emulsions, the diffusion coefficients for water are similar, though they are always higher for $1 \mathrm{wt} \% \mathrm{Na}$ caseinate emulsions. However, on increasing the Na-caseinate concentration to $3 \mathrm{wt} \%$ the water diffusion coefficients are significantly reduced to $\sim 22 \%$ lower than for $1 \mathrm{wt} \% \mathrm{Na}$ caseinate emulsions. This is indicative of enhanced oil droplet interactions and/or enhanced oil droplet/water interactions and/or oil droplet/solubilised protein/water interactions.

The diffusion coefficient of water was also measured using values of $\Delta$ up to $200 \mathrm{~ms}$. The measured coefficients at each value of $\Delta$ showed similar trends to those shown in Fig. 5.4 and Fig. 5.5 with little variation in the diffusion coefficients determined at each concentration of soybean oil and Na-caseinate. These results verify that water undergoes unrestricted diffusion at all soybean oil and Na-caseinate concentrations. 


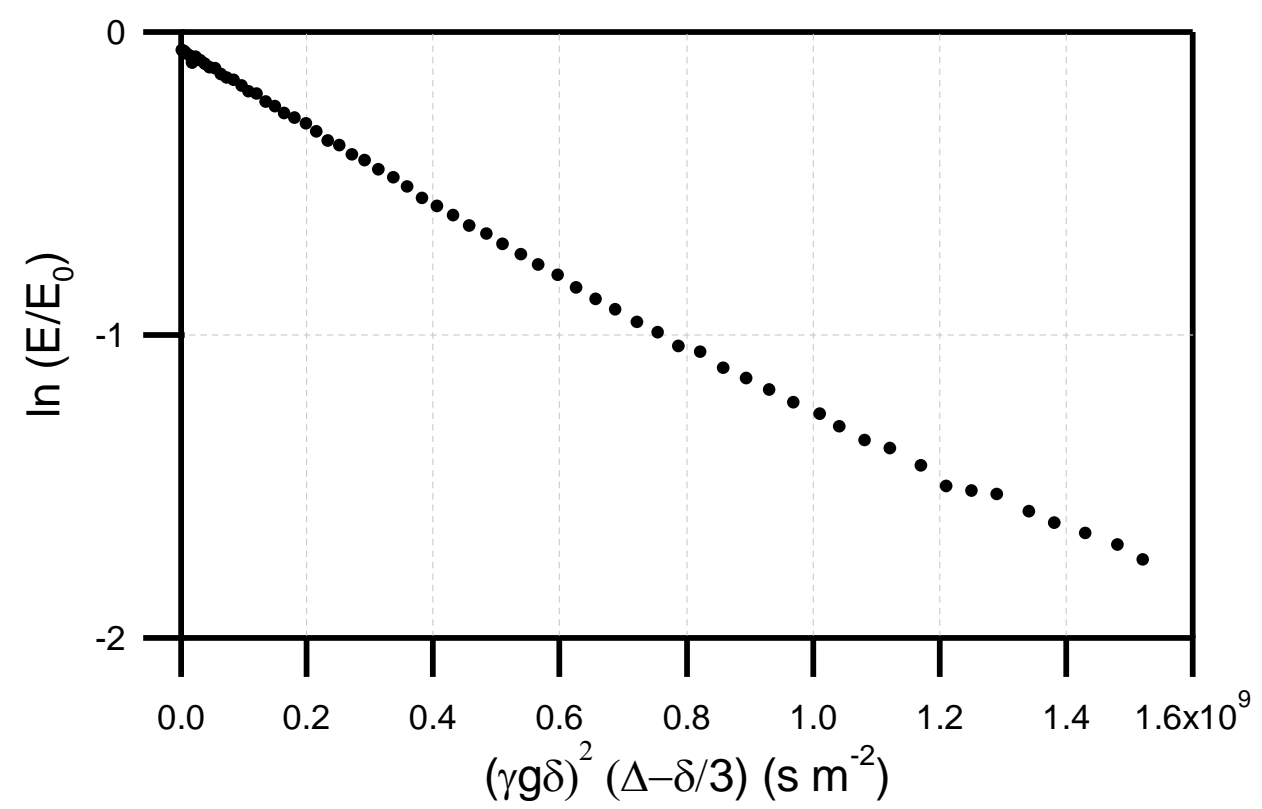

Figure 5.4: Echo attenuation plot for water diffusion in a freshly prepared $50 \mathrm{wt} \%$ soybean oil $/ 2 \mathrm{wt} \% \mathrm{Na}-$ caseinate $/ 48 \mathrm{wt} \%$ water emulsion, $\Delta=20 \mathrm{~ms}$.

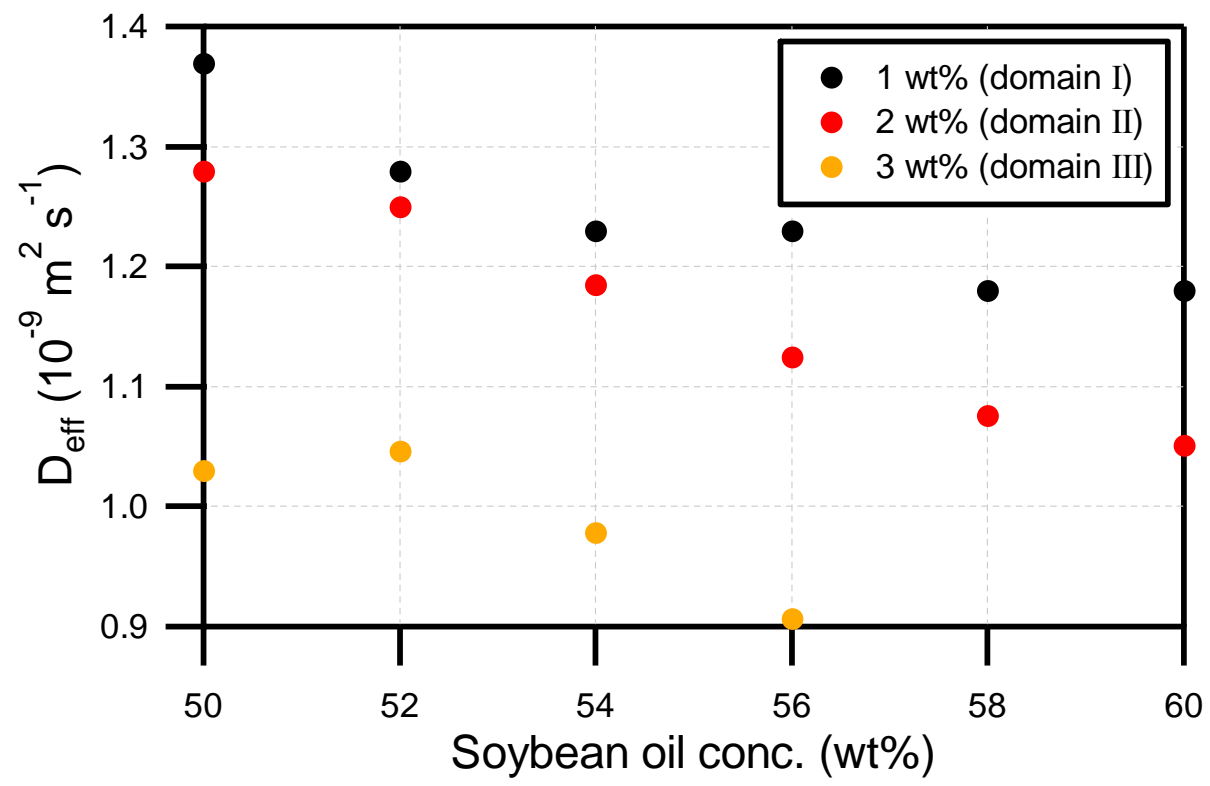

Figure 5.5: Diffusion coefficient of water as a function of increasing soybean oil concentration, $\Delta=20 \mathrm{~ms}$, for freshly prepared soybean oil/1, 2 and $3 \mathrm{wt} \% \mathrm{Na}$-caseinate/water emulsions.

In Fig. 5.6 are shown the diffusion coefficients of water as a function of Na-caseinate concentration, for $50 \mathrm{wt} \%$ soybean oil/Na-caseinate/water emulsions at $\Delta=20 \mathrm{~ms}$. The water diffusion coefficient remains approximately constant for Na-caseinate concentrations $\leq 1.5 \mathrm{wt} \%$, indicating the overall path travelled by water is not altered within this concentration range. At higher Na-caseinate concentrations, i.e., $1.75 \mathrm{wt} \%$, there is a 
significant drop to lower diffusion coefficients followed by a rapid decrease on further increasing Na-caseinate concentration. As such, an apparent change in emulsion microstructure occurs in the vicinity of $1.5 \mathrm{wt} \% \mathrm{Na}$-caseinate. This is consistent with the observed macroscopic phase transition, i.e., from domain I to II occurring at this Na-caseinate concentration in the phase diagram of this soybean oil system (see Fig. 4.2). The corresponding transition from domain II to III is not readily evidenced in the water diffusion data.

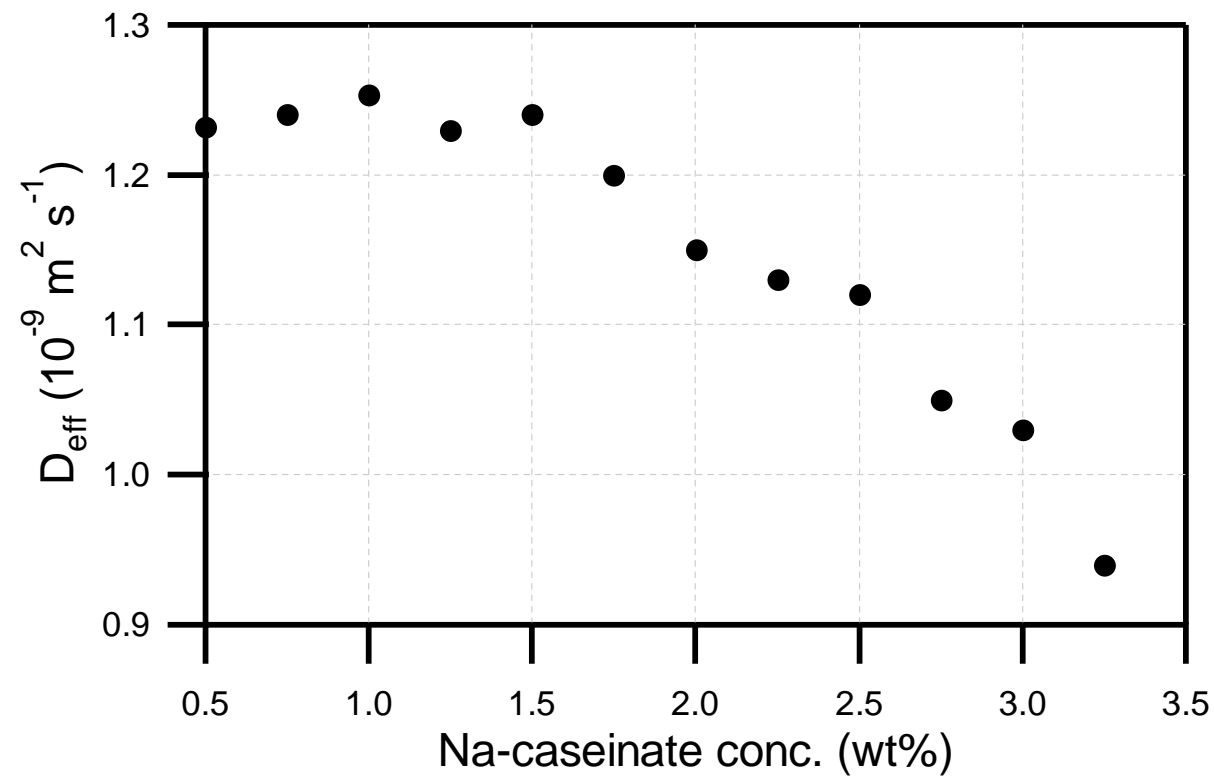

Figure 5.6: Diffusion coefficient of water as a function of increasing Na-caseinate concentration, $\Delta=20 \mathrm{~ms}$, for freshly prepared $50 \mathrm{wt} \%$ soybean oil/Na-caseinate/water emulsions.

The diffusion coefficient for water in all samples remains on the same order of magnitude as that of bulk water. This observation, coupled with the overall monotonic decrease of the water diffusion coefficient as shown in Fig. 5.5 confirms that these emulsions are of the oil-in-water type, where water is always the continuous phase.

For the soybean oil system, the measured oil attenuation data showed a multiexponential decay reflecting the polydisperse nature of the emulsion droplets and the existence of different types of oil-based diffusion. In Fig. 5.7 is shown a characteristic set of attenuation data, here for a $50 \mathrm{wt} \%$ soybean oil $/ 2 \mathrm{wt} \% \mathrm{Na}$-caseinate $/ 48 \mathrm{wt} \%$ water emulsion at $\Delta=200 \mathrm{~ms}$. 


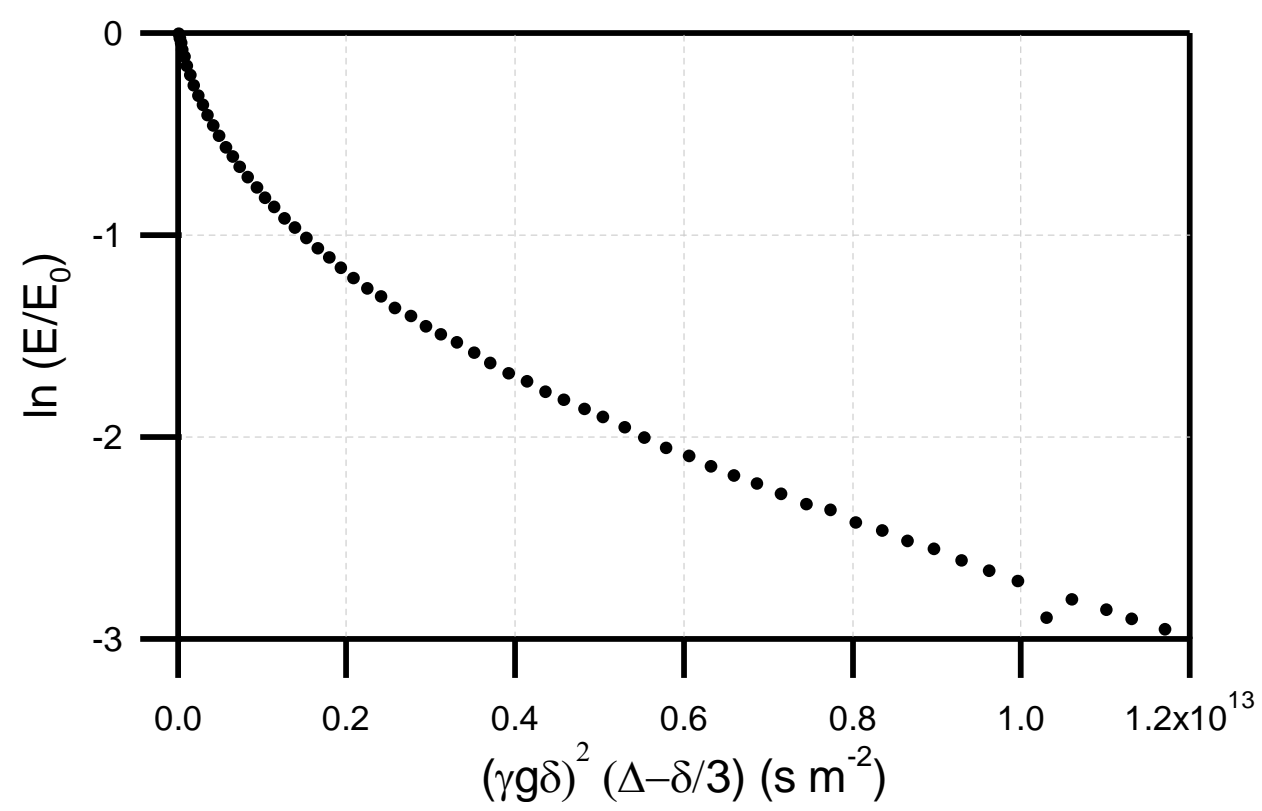

Figure 5.7: Echo attenuation plot for soybean oil diffusion in a freshly prepared $50 \mathrm{wt} \%$ soybean oil/2 wt $\% \mathrm{Na}-$ caseinate $/ 48 \mathrm{wt} \%$ water emulsion, $\Delta=200 \mathrm{~ms}$.

An inverse Laplace transformation was performed on the raw attenuation data using software developed by Callaghan et al. ${ }^{1}$, according to an algorithm first used by Provencher ${ }^{2}$. In Fig. 5.8 is shown the distribution of diffusion coefficients obtained from the analysis of the data presented in Fig. 5.7. Four distinct oil diffusion peaks exist with the diffusion coefficients spanning from approximately $6.7 \times 10^{-14}$ to $1.7 \times 10^{-11} \mathrm{~m}^{2} \mathrm{~s}^{-1}$. We denote the slowest to fastest oil diffusion peaks as peaks 1 to 4 . The unrestricted molecular diffusion coefficient of soybean oil is on the order of $10^{-10} \mathrm{~m}^{2} \mathrm{~s}^{-1}$. It is clear that soybean oil diffusion is highly restricted upon homogenisation with an up to 10000-fold decrease in diffusion coefficient being measured.

In order to facilitate the analysis of the data and to enable correlation between the data displayed in Fig. 5.8 and the types of diffusion occurring in the emulsion, dilution with aqueous phosphate buffer was incorporated prior to measurement with the gross assumption that the droplet size distribution is minimally perturbed. This dilution protocol was introduced with the specific aim of resolving which oil diffusion peaks are due to the restricted diffusion of the oil droplets. Fig. 5.9 shows the distribution of diffusion coefficients acquired at $\Delta=200 \mathrm{~ms}$ for four different emulsion to aqueous phosphate buffer volume ratios. 


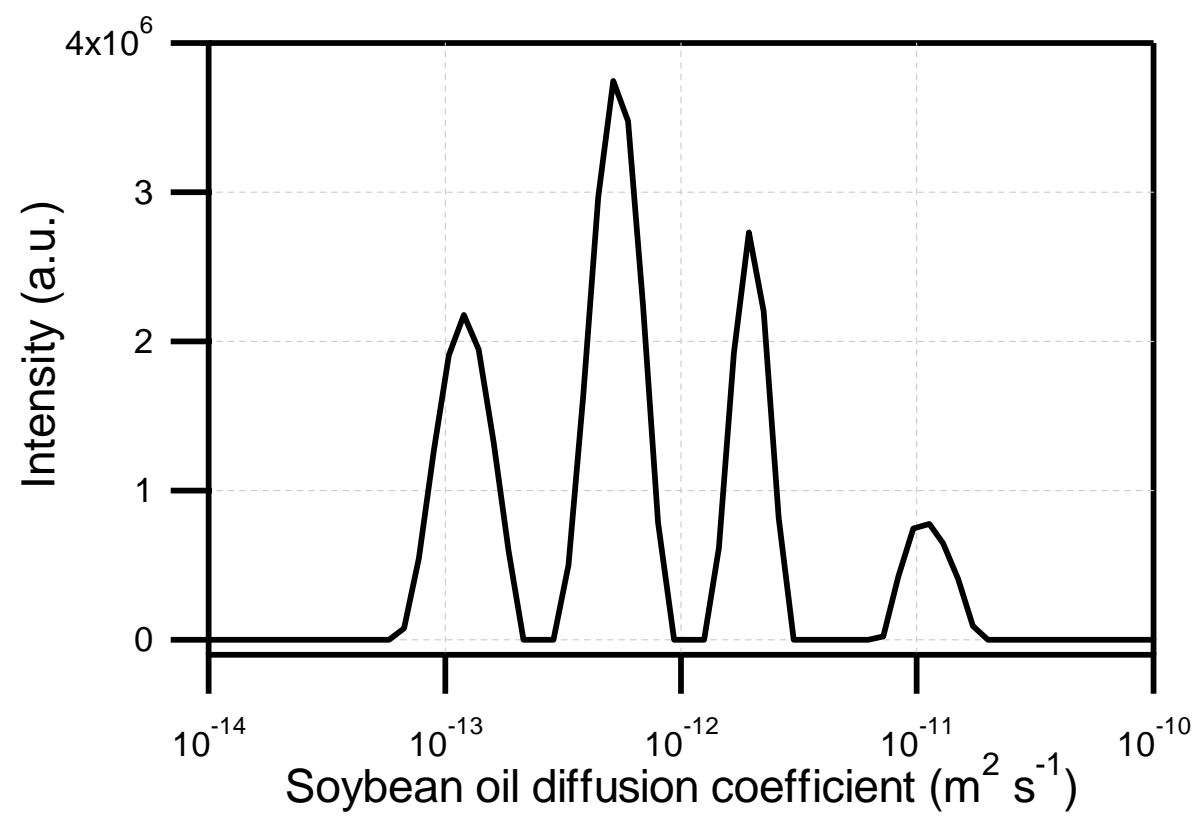

Figure 5.8: Soybean oil diffusion coefficient distribution for a $50 \mathrm{wt} \%$ soybean oil $/ 2 \mathrm{wt} \% \mathrm{Na}$-caseinate $/ 48 \mathrm{wt} \%$ water emulsion, attenuation data shown in Fig. 5.7, as obtained using an inverse Laplace transformation, $\Delta=$ $200 \mathrm{~ms}$.

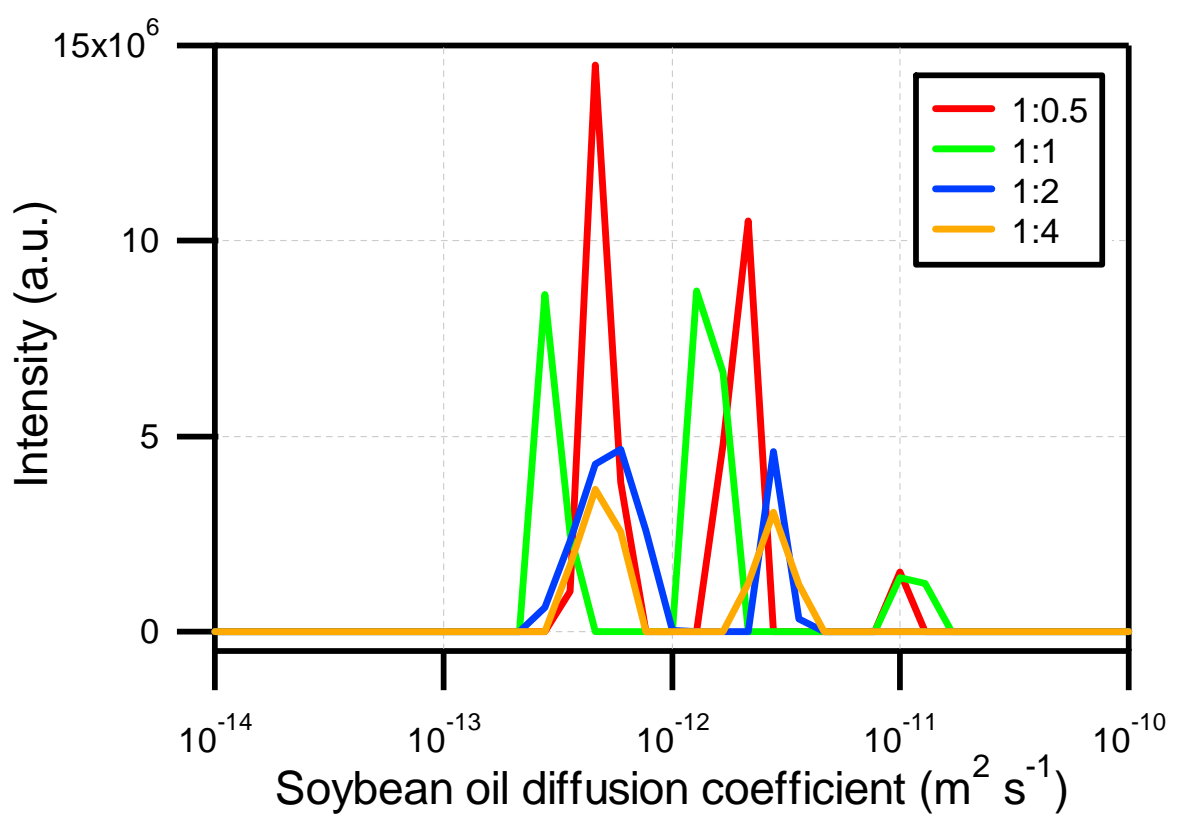

Figure 5.9: Soybean oil diffusion coefficient distributions for $50 \mathrm{wt} \%$ soybean oil $/ 2 \mathrm{wt} \% \mathrm{Na}$-caseinate $/ 48 \mathrm{wt} \%$ water emulsions obtained using an inverse Laplace transformation upon dilution at $\Delta=200 \mathrm{~ms}$ with emulsion to aqueous phosphate buffer volume ratios of 1:0.5, 1:1, 1:2 and 1:4.

It is evident that for an emulsion to aqueous phosphate buffer volume ratio as low as 1:0.5, the slowest (peak 1) oil diffusion peak seen in Fig. 5.8 is absent in Fig. 5.9. This oil diffusion peak was never observed upon further dilution and for all observation times, $\Delta$, 
used, up to $2200 \mathrm{~ms}$ (data not shown). Closer inspection of the diffusion coefficient distributions in Fig. 5.9 also indicates that the fastest (peak 4) oil diffusion peak in Fig. 5.8 disappears at emulsion to aqueous phosphate buffer volume ratio above 1:1. For peak 2 and peak 3 in Fig. 5.8, the diffusion coefficients obtained, corresponding to the peak maximum, for all dilution factors presented in Fig. 5.9 range from $\sim 3$ to $6 \times 10^{-13} \mathrm{~m}^{2} \mathrm{~s}^{-1}$ and from 1.3 to $\sim 3 \times 10^{-12} \mathrm{~m}^{2} \mathrm{~s}^{-1}$. The changes of the two oil diffusion peaks at emulsion to aqueous phosphate buffer volume ratios of 1:2 and 1:4 are not as dramatic as for the initial dilution.

In addition to the dilution experiments which aid in some differentiation and assignment of the four oil diffusion peaks shown in Fig. 5.8, experiments performed on the undiluted emulsions as a function of experimental observation time, $\Delta$, allowed determination of characteristic times and therefore length scales of the system. In Fig. 5.10 are exhibited the diffusion coefficients extracted at the maximum peak position for each of the four oil diffusion peaks shown in Fig. 5.8 as a function of increasing $\Delta$ for $50 \mathrm{wt} \%$ soybean oil $/ 2 \mathrm{wt} \%$ Na-caseinate $/ 48 \mathrm{wt} \%$ water emulsions. The product of $D \cdot \Delta$, on the right hand side of each figure, aids the interpretation for each oil diffusion peak. An increasing or constant $D \cdot \Delta$ reflects unrestricted or restricted diffusion, respectively. In the case of restricted diffusion, we anticipate that coalescence is not occurring within the timeframe of the experiment, i.e., the oil droplets collide, interact and then move away from each other but do not fuse.

The diffusion coefficient corresponding to peak 1 continuously decays with increasing $\Delta$, indicating that some kind of restricted diffusion is being measured. Note that $\Delta$ values up to a maximum of $800 \mathrm{~ms}$ only could be used. For times longer than this the lower limit of the instrument, $D \sim 10^{-14} \mathrm{~m}^{2} \mathrm{~s}^{-1}$, was reached. Hence, for longer $\Delta, D$ appeared to be constant but this is an erroneous result. Correspondingly the product of $D \cdot \Delta$ is constant verifying that restricted diffusion of oil droplets in the size range of $\sim 0.3$ to $5 \mu \mathrm{m}$ (see bimodal distribution in Fig. 5.1) is being probed. These data confirm the assignment of this oil diffusion peak from the dilution experiments (see Fig. 5.8 and Fig. 5.9).

For peak 2, with increasing observation time, $\Delta$, up to $\sim 1 \mathrm{~s}$, the diffusion coefficient decreases continuously. $D$ remains nearly constant for $\Delta$ longer than $800 \mathrm{~ms}$. It is noticeable however that the product of $D \cdot \Delta$ is constant for $\Delta<200 \mathrm{~ms}$ but an increasing function for $\Delta$ longer than $200 \mathrm{~ms}$. The diffusion coefficients corresponding to peak 3 exhibit a very similar trend as those observed in the diffusion data for peak 2. The transition in the diffusion coefficient trend occurs at $\Delta \sim 700 \mathrm{~ms}$ while for the $D \cdot \Delta$ data the transition remains at $\Delta \sim$ $200 \mathrm{~ms}$. For peak 4, again, with increasing observation time, the diffusion coefficient 
displayed a continuous decay for $\Delta$ up to $\sim 650 \mathrm{~ms}$. The product of $D \cdot \Delta$ did not appear to be constant but instead increased with increasing observation time for all values of $\Delta$.
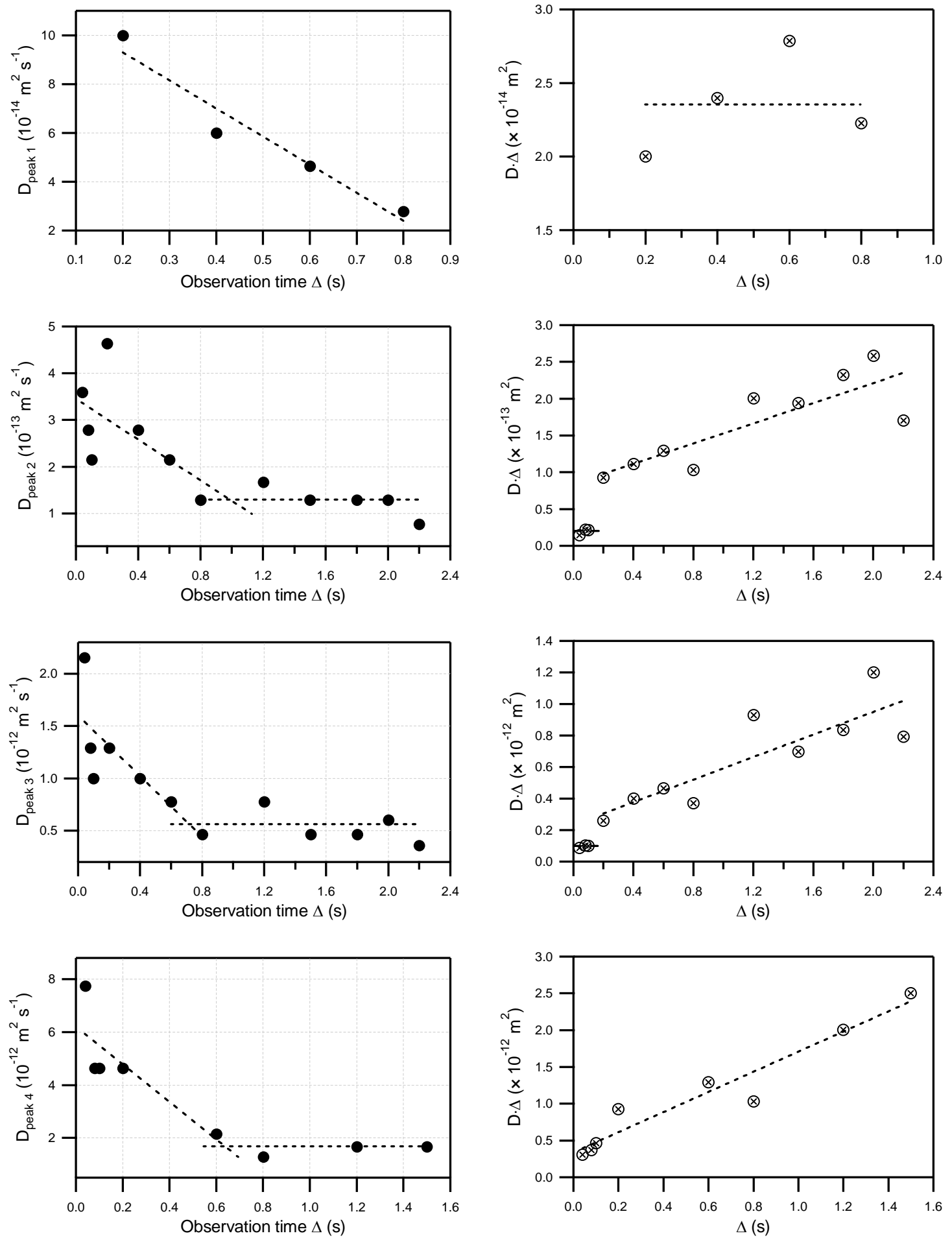

Figure 5.10: Soybean oil diffusion coefficients as a function of increasing observation time, $\Delta$, for $50 \mathrm{wt} \%$ soybean oil $/ 2 \mathrm{wt} \% \mathrm{Na}$-caseinate $/ 48 \mathrm{wt} \%$ water emulsions obtained by performing an inverse Laplace transformation. Each figure represents the diffusion coefficients for one of the four different oil diffusion peaks shown in Fig. 5.8 at its peak maximum with the corresponding product of $D \cdot \Delta$ on the right hand side. 
The effect of increasing soybean oil concentration, at a fixed Na-caseinate concentration, on the slowest oil diffusion peak (i.e., that due to the restricted diffusion of the oil droplets) was studied (Fig. 5.11). Note that the extracted diffusion coefficients for $1 \mathrm{wt} \%$ and $2 \mathrm{wt} \% \mathrm{Na}$-caseinate overlap with each other at the soybean oil concentration of 50,52 and $56 \mathrm{wt} \%$. As soybean oil concentration is increased, the diffusion coefficients of the slowest oil diffusion peak for all Na-caseinate concentrations studied fluctuate around 1 to 3 $\times 10^{-13} \mathrm{~m}^{2} \mathrm{~s}^{-1}$, as was seen in Fig. 5.11 (a). This is particularly true for emulsions prepared with $2 \mathrm{wt} \%$ Na-caseinate. Within the experimental uncertainty, the measured diffusion coefficients at a fixed Na-caseinate concentration are approximately constant.

The experimental uncertainty is contributed by three main factors. 1) The measured oil diffusion coefficients are of the order of $10^{-13} \mathrm{~m}^{2} \mathrm{~s}^{-1}$ and are close to the limit of the measurable diffusion coefficient $\left(\sim 10^{-14} \mathrm{~m}^{2} \mathrm{~s}^{-1}\right)$ and the sensitivity of the instrument is diminished enhancing experimental uncertainty. 2) The inherent polydispersity of the system. Unlike monodisperse systems, the measured attenuation is an overall signal contributed by the phase shift and magnetisation change of spins of different size molecules. For a total attenuation of $\sim 10 \%$, significantly less than $10 \%$ attenuation is achieved for any particular oil droplet size within the sample. 3) The inverse Laplace transformation. For the analysis of oil diffusions, the distribution of diffusion coefficients is sensitive to the value of the smoothing parameter $\alpha$ used in the inverse Laplace transformation. Furthermore, two oil diffusion peaks that are close to each other (less than an order of magnitude separation) are poorly distinguished using an inverse Laplace transformation. Therefore, the oil diffusion peaks in a distribution might be over- or underemphasised in intensity and shifted in terms of absolute value.

From the three contributions to uncertainty mentioned above, the uncertainty in the oil diffusion coefficients extracted from the distribution is $\sim 20 \%$. For unrestricted diffusion of a monodisperse sample, a value of $\sim 5 \%$ is more appropriate.

The values of the diffusion coefficients for $1 \mathrm{wt} \% \mathrm{Na}$-caseinate emulsions are slightly higher than those obtained for the remainder of the series. This indicates that the diffusion of oil droplets is slightly less restricted in these emulsions. This is supported by the relative intensity of the slowest oil diffusion peak extracted from inverse Laplace transformation shown in Fig. 5.11 (b). At a fixed soybean oil concentration, the relative intensity of the slowest oil diffusion peak for emulsions prepared with $1 \mathrm{wt} \% \mathrm{Na}$-caseinate is slightly lower compared with emulsion prepared at $2 \mathrm{wt} \%$ and $3 \mathrm{wt} \%$ Na-caseinate. As soybean oil 
concentration is increased, the relative intensity of the slowest oil diffusion peak is increased for all Na-caseinate concentrations as expected with more oil droplets now present enhancing restricted diffusion of the oil droplets. All extracted values fall within the same order of magnitude.
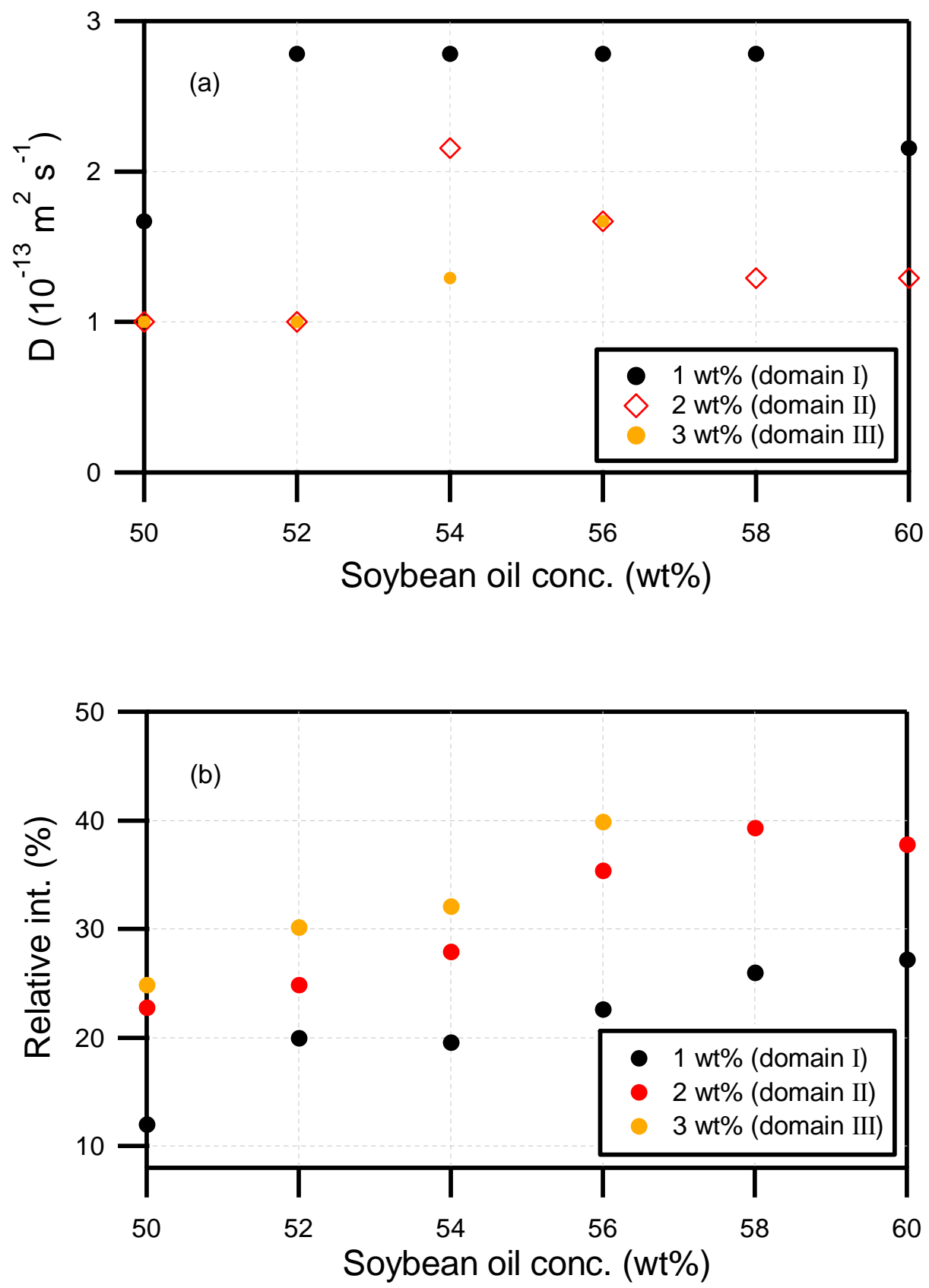

Figure 5.11: (a) Diffusion coefficient of the slowest oil diffusion peak (estimated at the peak maximum) as a function of increasing soybean oil concentration, $\Delta=200 \mathrm{~ms}$, for freshly prepared soybean oil/1, 2 and $3 \mathrm{wt} \%$ Na-caseinate/water emulsions. (b) Corresponding relative intensity of the slowest oil diffusion peak as a function of increasing soybean oil concentration. 
In Fig. 5.12 is shown the relative intensity of the slowest oil diffusion peak on increasing Na-caseinate concentration for $50 \mathrm{wt} \%$ soybean oil/Na-caseinate/water emulsions. Three distinct regions can be identified. Within the experimental uncertainty, at low Nacaseinate concentration with the cut off at around $1.5 \mathrm{wt} \%$, the relative intensity of the slowest oil diffusion peak is constant and rises steadily at higher Na-caseinate concentration before finally again flattening out at approximately $2.25 \mathrm{wt} \%$ of Na-caseinate. This observed variation in relative intensity of the slowest oil diffusion peak correlates well with the different macroscopic stability presented in the phase diagram in Chapter 4, corresponding to domains I, II and III.

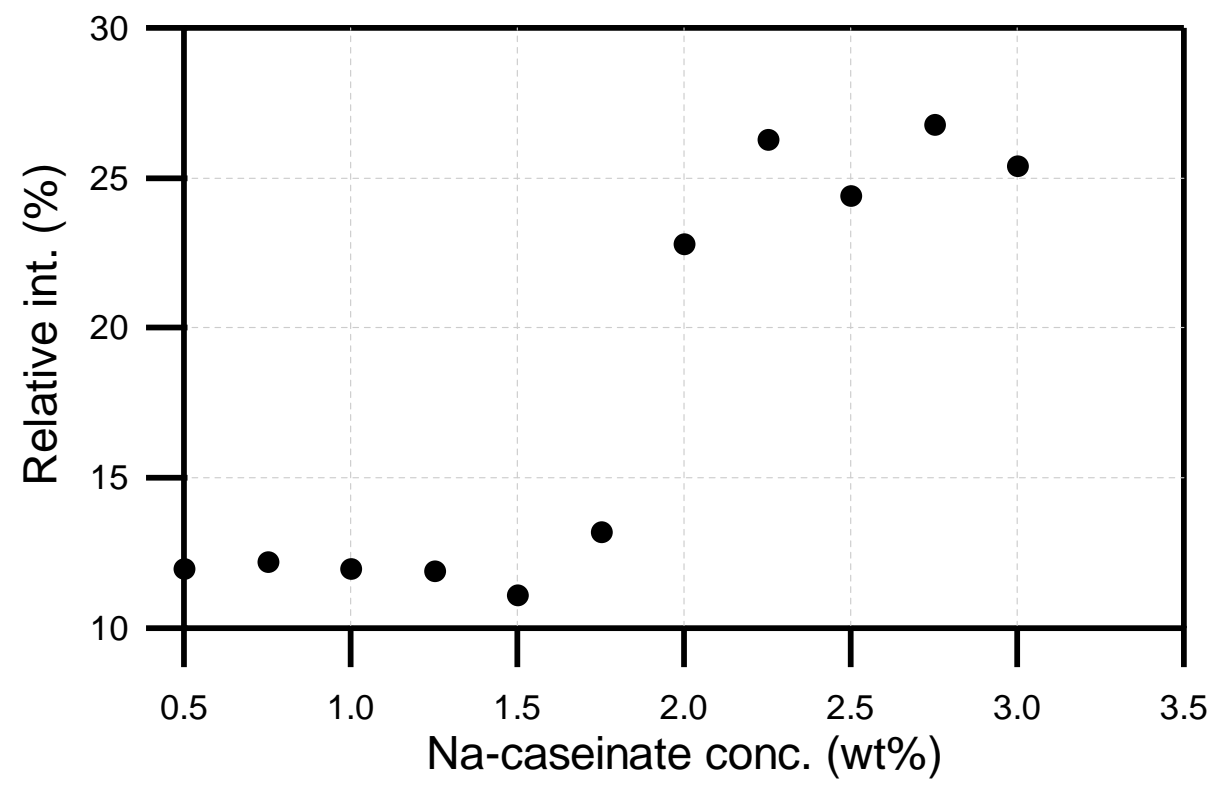

Figure 5.12: Relative intensity of the slowest oil diffusion peak as a function of increasing Na-caseinate concentration, $\Delta=200 \mathrm{~ms}$, for freshly prepared $50 \mathrm{wt} \%$ soybean oil/Na-caseinate/water emulsions.

\subsubsection{Rheology}

The results in this section present how the properties and microstructure, such as the packing of oil droplets and the extent of deformation, influence the flow behaviour of emulsions. In Fig. 5.13 is shown three representative plots of the response of the emulsions in domains I, II and III as a function of strain amplitude. At small strains, both $G^{\prime}$ and $G^{\prime \prime}$ for all samples are relatively constant. $G^{\prime}$ is approximately one order of magnitude larger than $G^{\prime \prime}$, indicating that the emulsions behave as elastic solids. Moving from domain I to III, both $G^{\prime}$ and $G^{\prime \prime}$ of the emulsions increase. The point where $G^{\prime}$ crosses $G^{\prime \prime}$ separates the linear regime from the 
non-linear regime and corresponds to a value of strain amplitude spanning from around $20 \%$ to $70 \%$. Above the cross over point the sample flows like a fluid.

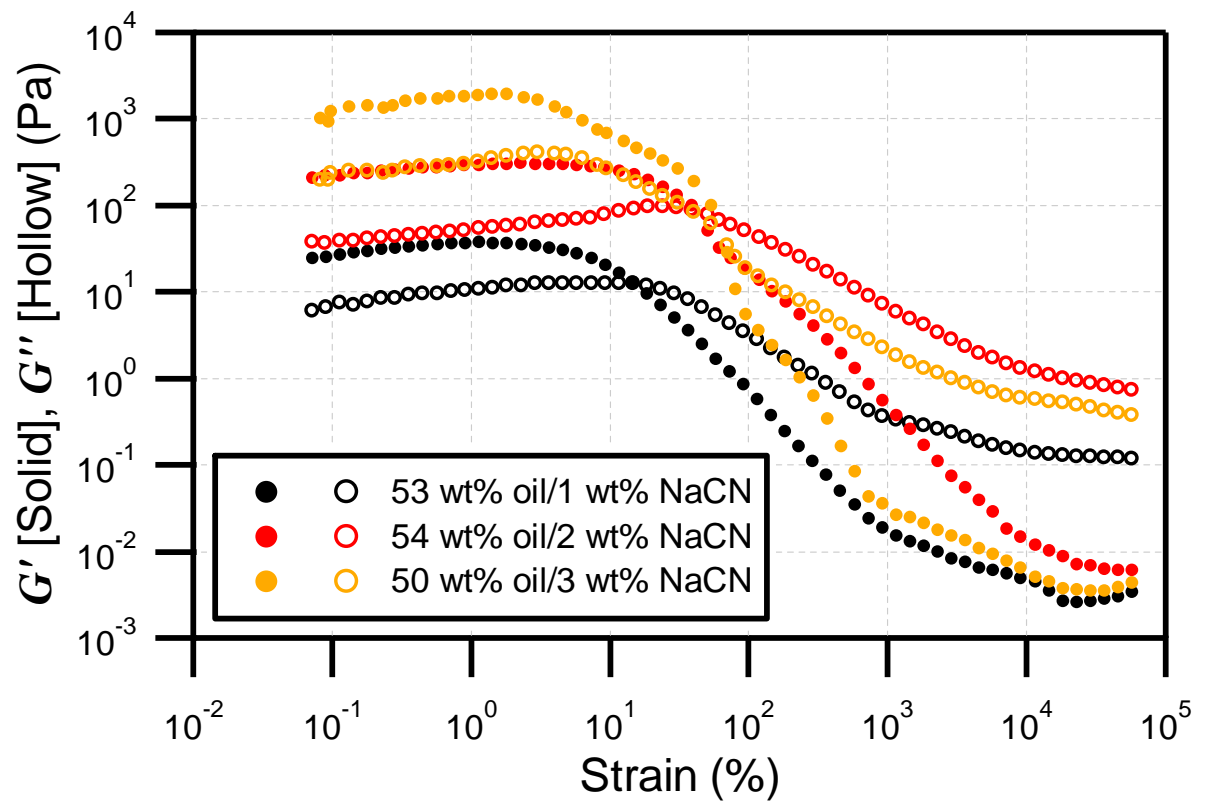

Figure 5.13: Representative log-log plots of the strain amplitude dependence of the storage and loss moduli, $G^{\prime}$ and $G^{\prime \prime}$ at $1 \mathrm{rad} \mathrm{s}^{-1}$, for freshly prepared soybean oil/Na-caseinate/water emulsions. We denote $\mathrm{NaCN}$ as Nacaseinate.

Linear viscoelastic measurements of soybean oil $/ 2,3 \mathrm{wt} \%$ Na-caseinate/water emulsions were carried out by applying an oscillatory shear of constant amplitude of $2 \%$. The data are shown in Fig. 5.14 left and right, respectively. $G^{\prime}$ lies above $G^{\prime \prime}$ for all samples and the entire range of frequencies investigated. Both $G^{\prime}$ and $G^{\prime \prime}$ exhibit a weak power law dependence. The fits yield $G^{\prime} \propto \omega^{0.095 \pm 0.012}$ and $G^{\prime \prime} \propto \omega^{0.065 \pm 0.011}$ for $2 \mathrm{wt} \%$ and $G^{\prime}$ $\propto \omega^{0.108 \pm 0.025}$ and $G^{\prime \prime} \propto \omega^{0.073 \pm 0.016}$ for $3 \mathrm{wt} \%$ Na-caseinate systems, respectively. The viscoelastic responses of the two state diagrams are very similar. $G^{\prime}$ and $G^{\prime \prime}$ are separated by approximately an order of magnitude and are unaffected by increasing soybean oil concentration. However, the values of $G^{\prime}$ and $G^{\prime \prime}$ for emulsions prepared with $3 \mathrm{wt} \% \mathrm{Na}$ caseinate are slightly higher than those prepared with $2 \mathrm{wt} \% \mathrm{Na}$-caseinate.

Given that the concentration of soybean oil does not alter the viscoelastic response of the emulsions, this was maintained constant while the Na-caseinate concentration was varied. The viscoelastic behaviour of $50 \mathrm{wt} \%$ soybean oil/Na-caseinate/water emulsions on incrementing Na-caseinate concentration is given in Fig. 5.15. As observed in Fig. 5.14, G' and $G^{\prime \prime}$ are separated by approximately an order of magnitude over the full frequency range. 
Emulsions prepared with $1 \mathrm{wt} \%$ Na-caseinate show a stronger power law dependence $\left(G^{\prime}\right.$ and $\left.G^{\prime \prime} \propto \omega^{0.23 \pm 0.008}\right)$ compared with the other emulsions but otherwise the responses are similar. Both $G^{\prime}$ and $G^{\prime \prime}$ increase on increasing Na-caseinate concentration, reflecting a difference in the microstructure and interfacial domain between the emulsions and an enhancement in the viscosity, which is a characteristic of concentrated emulsions ${ }^{3,4}$.
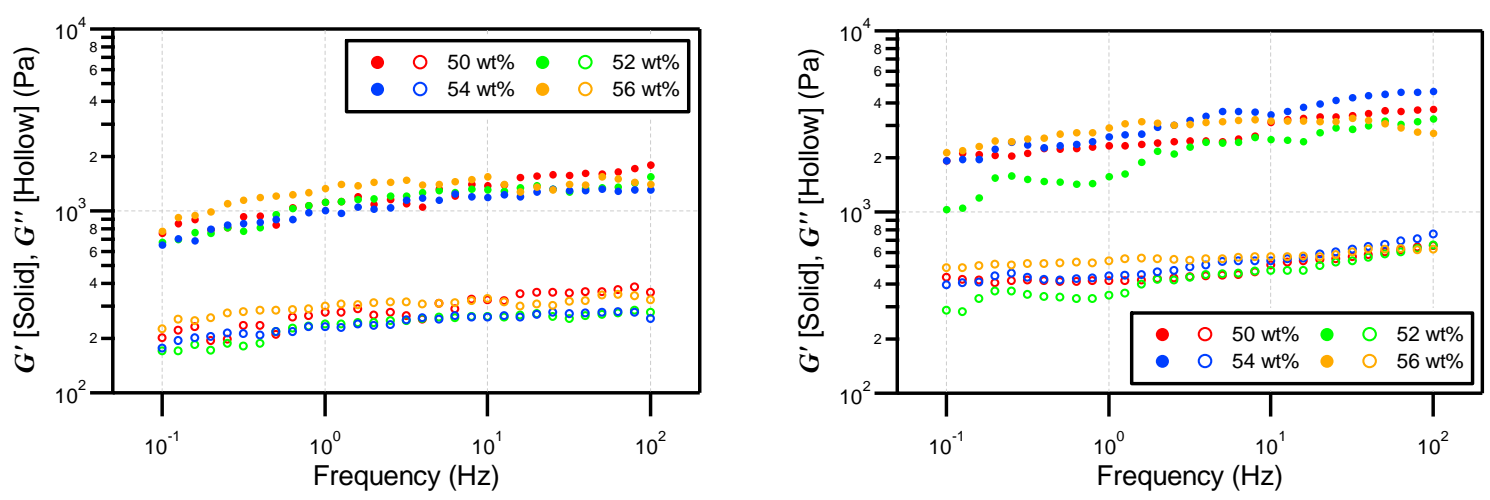

Figure 5.14: Log-log plot of the frequency dependence of $G^{\prime}$ and $G^{\prime \prime}$ at a strain amplitude of $2 \%$, for freshly prepared soybean oil/2 (left, domain II), 3 wt\% (right, domain III) Na-caseinate/water emulsions.

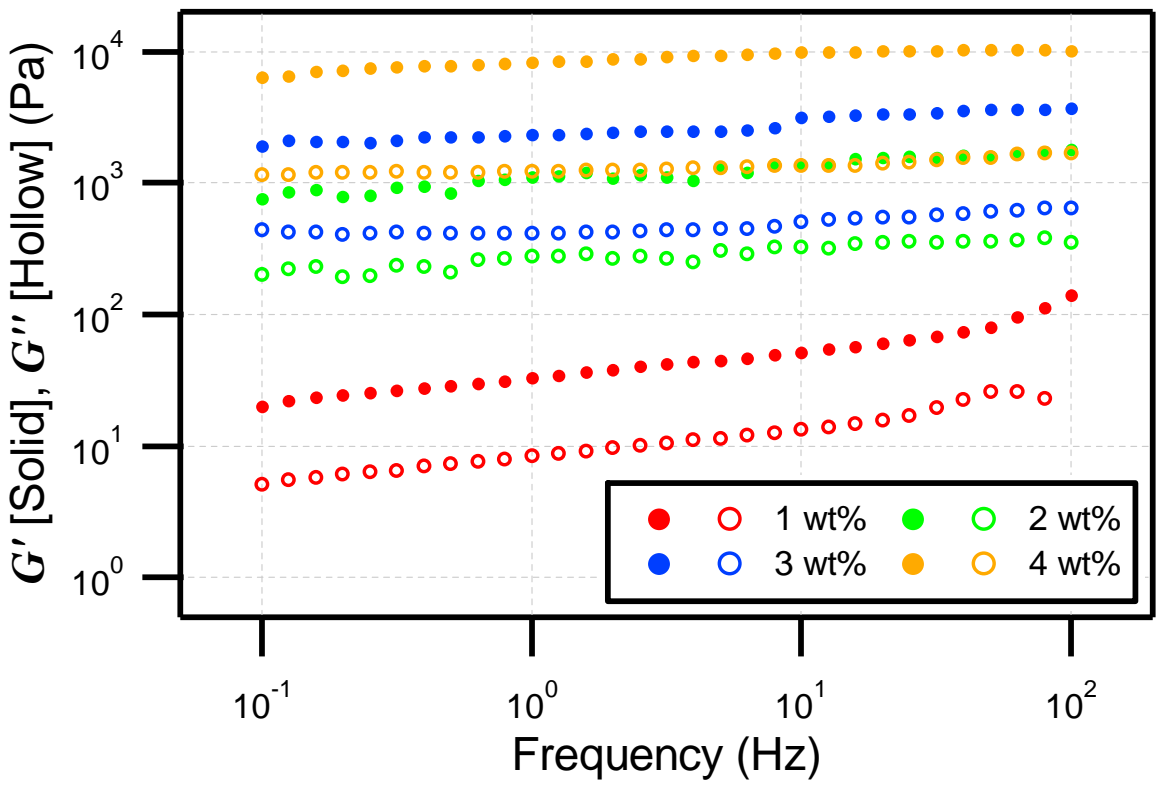

Figure 5.15: Log-log plot of the frequency dependence of $G^{\prime}$ and $G^{\prime \prime}$ at a strain amplitude of $2 \%$ on increasing Na-caseinate concentration, for freshly prepared $50 \mathrm{wt} \%$ soybean oil/Na-caseinate/water emulsions.

\subsubsection{Cryo-SEM}

In Fig. 5.16 (a) is shown a representative overview of the microstructure of a $50 \mathrm{wt} \%$ soybean 
oil $/ 2 \mathrm{wt} \%$ Na-caseinate $/ 48 \mathrm{wt} \%$ water emulsion. The microstructure shows a polydisperse dispersion of oil droplets in a water continuum, as expected from the light scattering and the PGSTE-NMR results. Generally, oil droplets are spherical in shape. Oil droplets (e.g., those circled in yellow in Fig. 5.16 (b)) are often squeezed together deforming the interfacial region whereas water exists as large flat regions (e.g., see regions marked by red arrows) throughout the system revealing the inherent inhomogeneity of all emulsions. The micrograph shown in Fig. 5.16 (c) indicates the presence of casein subaggregates at the oil/water interfaces and in the water region with an average diameter spanning from 10 to $50 \mathrm{~nm}$.

In Fig. 5.17 are representative micrographs exhibiting the microstructure of soybean oil/Na-caseinate/water emulsions in the five different domains in the phase diagram (see Fig. 4.2) obtained after sublimation. Sublimation reveals the three-dimensional structure of the emulsions. Each of the domains is readily identified through a different microstructure. On increasing Na-caseinate and/or soybean oil concentrations sample viscosity and stickiness is increased, as such it was not possible to obtain cryo images for Na-caseinate concentrations above $5 \mathrm{wt} \%$ at the lowest soybean oil concentrations or above $60 \mathrm{wt} \%$ soybean oil at the lowest Na-caseinate concentrations, as samples could not be housed in the cryo sample holder. All micrographs are in good agreement with the light scattering and the PGSTENMR results showing a polydisperse distribution of oil droplets ranging in size from $\sim 100$ $\mathrm{nm}$ to $3 \mu \mathrm{m}$ with water existing as the continuous phase.

Fig. 5.17 (a), (b), (c) and (d) are micrographs for emulsions at fixed soybean oil concentration $(50 \mathrm{wt} \%)$ but increasing Na-caseinate concentration $(0.5,2,3$ and $5 \mathrm{wt} \%$, respectively, corresponding to domains I, II and III, the $5 \mathrm{wt} \%$ sample is at the border to domain V). Comparison of the micrographs show that the microstructure of the emulsions changes dramatically on increasing Na-caseinate concentration. Consider emulsions in domain I, it can be seen in Fig. 5.17 (a) that bridging occurs where the oil droplets join with adjacent oil droplets sharing the thin interfacial film. Single discrete oil droplets are difficult to identify. The thin interfacial layer posses a low repulsive barrier and therefore the emulsions in this domain rapidly phase separate. Extensive water domains are evident.

In contrast, for the $2 \mathrm{wt} \% \mathrm{Na}$-caseinate system (domain II in the phase diagram), as shown in Fig. 5.17 (b), the identity of individual droplets is largely preserved with good coverage of the proteins at the oil droplet surfaces, resulting in a high repulsive barrier due to steric and electrostatic stabilisation and increased emulsion stability. 

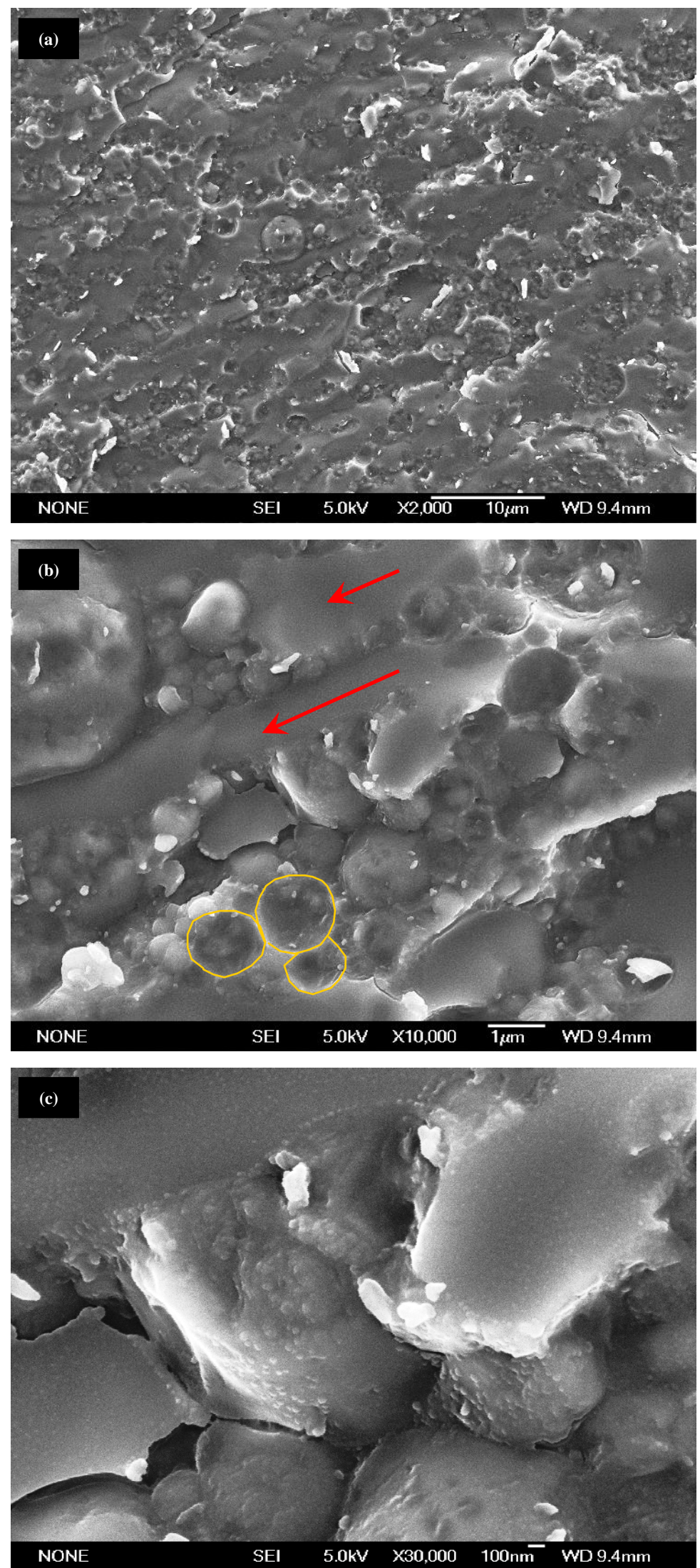

Figure 5.16: Cryo-SEM micrographs of (a) 2000, (b) 10000 and (c) 30000× magnification at the same spot for a freshly prepared $50 \mathrm{wt} \%$ soybean oil $/ 2 \mathrm{wt} \% \mathrm{Na}$-caseinate $/ 48 \mathrm{wt} \%$ water emulsion. Examples of water and oil domains are indicated by red arrows and yellow circles, respectively. 

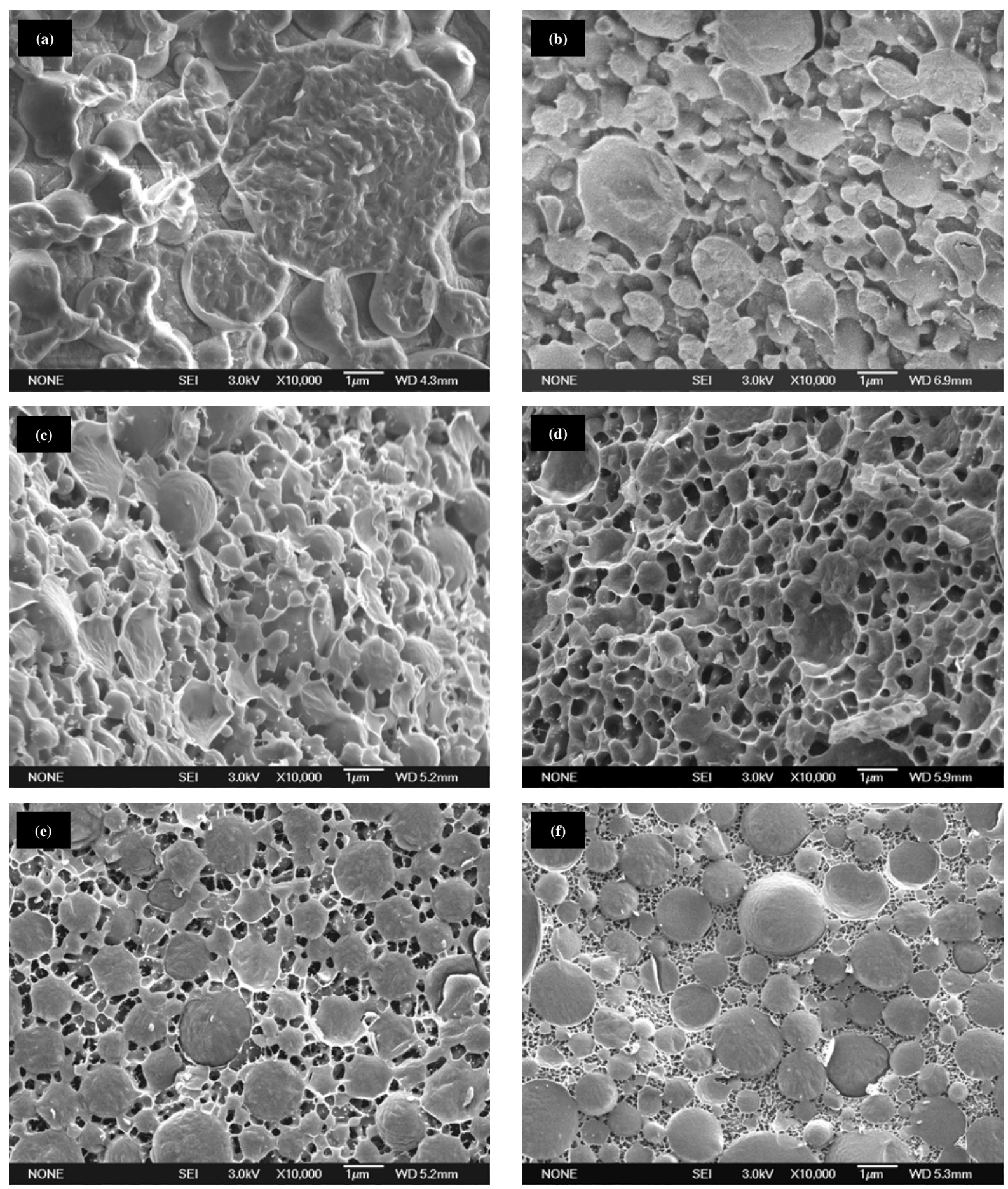

Figure 5.17: Cryo-SEM micrographs at 10000× magnification of freshly prepared $50 \mathrm{wt} \%$ soybean oil/(a): 0.5 (domain I), (b): 2 (domain II), (c): 3 (domain III) and (d): 5 (domain III bordering to domain V) wt\% Nacaseinate/water emulsions, (e): domain IV bordering to domain V, $53 \mathrm{wt} \%$ soybean oil/4 wt\% Na-caseinate/43 $\mathrm{wt} \%$ water emulsion and (f): domain $\mathrm{V}$, obtained after 30 minutes sublimation at $-110^{\circ} \mathrm{C}$.

In Fig. 5.17 (c) and (d) is shown the emulsion microstructure of domain III in the phase diagram. Again, the oil droplets are connected to each other but in a different way as compared with the 1 and 2 wt $\%$ Na-caseinate systems. Considerable connections between 
the oil droplets are evident, but unlike in domain I boundaries are easily resolved and the connections are a result of an excess of Na-caseinate, consistent with the occurrence of extensive depletion flocculation. Depletion flocculation counters the repulsive barrier to phase separation resulting in emulsions with reduced stability. Indeed emulsions within this domain are the least stable, destabilising within 1 day. As the concentration of protein is increased within this domain a more extensive network connecting oil droplets is evident (Fig. 5.17 (d)).

The microstructure of domain IV bordering to domain V (Fig. 5.12 (e)) is very similar to that seen at the limit of domain III. The higher concentration of oil droplets is evident which may be enough to shift the stability of these emulsions to be significantly greater than those emulsions in domain III brought about by enhancement of the rudimentary foundations of the full three-dimensional network present in domain $\mathrm{V}$.

Fig. 5.17 (f) is a representative micrograph of the emulsions within domain $\mathrm{V}$ in the phase diagram exhibiting a distinct microstructure. Discrete oil droplets are easily noticeable and are extensively linked to each other via an interaggregate three-dimensional network in the continuous phase. This network is a result of protein self-assembly. This microstructure leads to enhanced viscosity and high stability of the emulsions. Such network formation has been shown to occur in Na-caseinate dispersions for Na-caseinate concentration as low as 6 wt $\%$ (see Chapter 3). Formation of a Na-caseinate three-dimensional network within the continuous aqueous phase is consistent with the enhanced viscosity of emulsions in this domain, the resistance of the system to phase separate (emulsions are stable for $\sim 3$ weeks) and the eventual formation of a gel-like paste at periphery of the emulsion phase space.

\subsection{Discussion}

\subsubsection{Laser Diffraction Particle Sizing}

Presented in Fig. 5.18 is a log-log depiction of the mean radii of the lower and higher droplet modes as a function of time, extracted from the droplet size distribution of a $50 \mathrm{wt} \%$ soybean oil $/ 2 \mathrm{wt} \%$ Na-caseinate $/ 48 \mathrm{wt} \%$ water emulsion (see for example Fig. 5.3). Emulsion destabilisation $^{5,6}$ via the mechanism of either flocculation ${ }^{7}$ or coalescence results in phase separation and oil droplet growth as observed. 

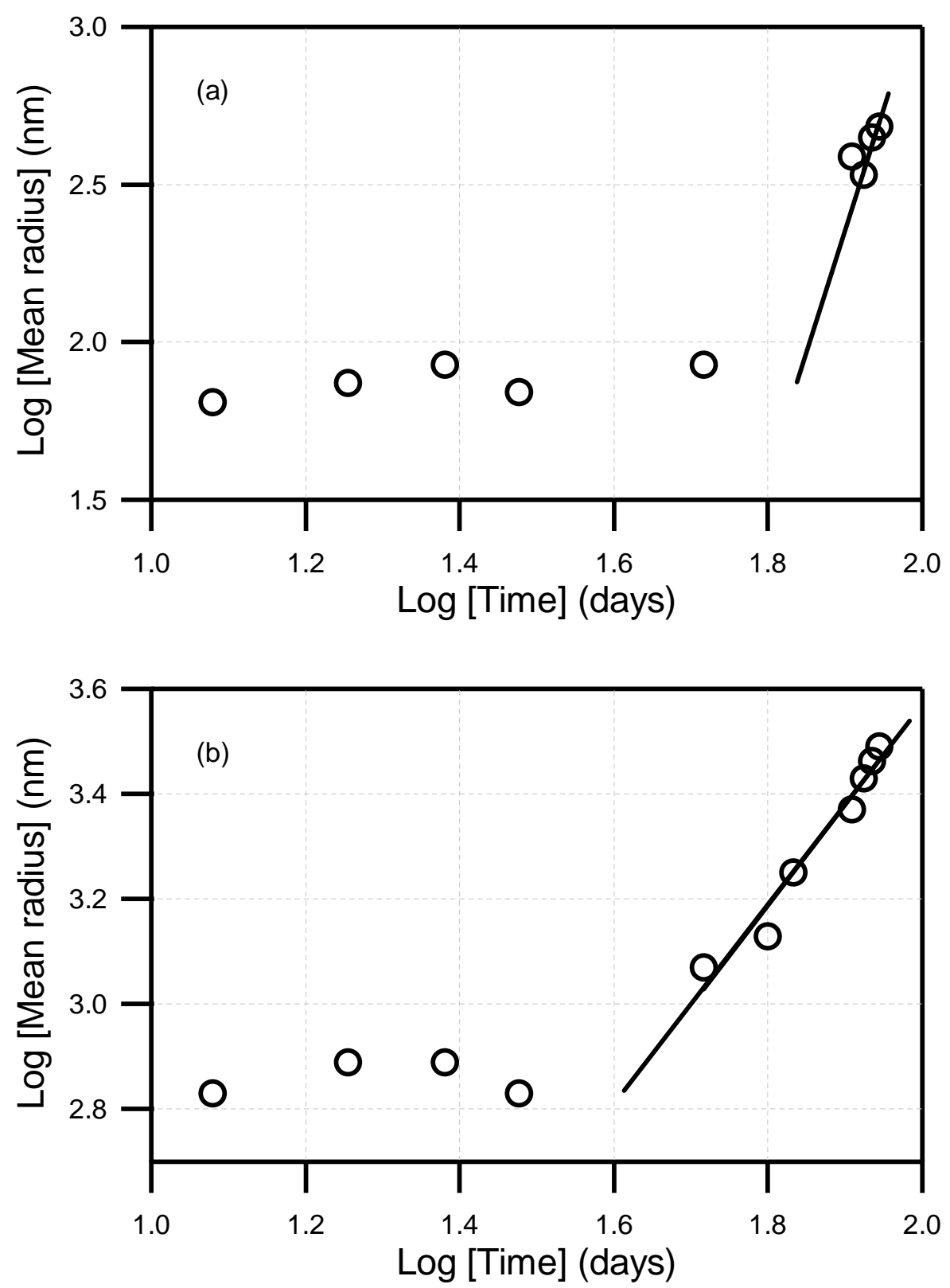

Figure 5.18: Mean radius of (a) lower and (b) higher droplet modes of a $50 \mathrm{wt} \%$ soybean oil $/ 2 \mathrm{wt} \% \mathrm{Na}$ caseinate $/ 48 \mathrm{wt} \%$ water emulsion over time. The fits describe subsequent to oil droplet growth (a) cubic and (b) square dependence of the lower and higher droplet modes, corresponding to destabilisation via standard and enhanced Ostwald ripening, respectively.

In a polydisperse system, the concentration of free oil molecules surrounding the smaller oil droplets is higher than that surrounding the larger oil droplets. The concentration gradient creates a flow. Thus, the small oil droplets flow towards the larger oil droplets ${ }^{8}$. This process known as standard Ostwald ripening ${ }^{9}$ is one of the mechanisms of depletion flocculation. The dispersed phase appears to be solubilised in the continuous phase before 
merging with other oil droplets. Transport of the dispersed phase through the continuous phase is expressed as ${ }^{10-13}$ :

$$
r_{t}^{3}-r_{t=0}^{3}=\omega t
$$

with

$$
\omega=\frac{8 c_{(\infty)} \gamma V_{m}^{2} D}{9 R T} .
$$

Hence, the rate of standard Ostwald ripening is essentially a cubic relation:

$$
\frac{d r^{3}}{d t}=\omega_{3}
$$

where $r_{t}$ is the mean oil droplet radius at time $t, r_{t=0}$ is the initial mean oil droplet radius, $\omega_{3}$ is the Ostwald ripening rate, $c_{(\infty)}$ is the oil solubility in an infinite sized oil droplet, $\gamma$ is the interfacial tension, $V_{m}$ is the oil molar volume, $D$ is the dispersed phase diffusion coefficient, $R$ is the gas constant and $T$ is the absolute temperature.

Enhanced Ostwald ripening is an acceleration of Ostwald ripening ${ }^{14,15}$. Here, the emulsifier molecules at the oil/water interface and in the continuous phase aid in the merging between oil droplets. Hence, destabilisation occurs more rapidly. This results in the following relationship ${ }^{16-18}$ :

$$
\frac{d r^{2}}{d t}=\omega_{2}
$$

An enhancement factor, $\varphi=\frac{C_{\infty, m} \cdot D_{m}}{C_{\infty, w} \cdot D_{w}}$ where $C_{\infty, m}$ and $C_{\infty, w}$ are the solubility of oil in, for example, the micelles and in the continuous phase (in our case an aqueous phosphate buffer), respectively and $D_{m}$ and $D_{w}$ are the diffusion of the micelle and the oil in the continuous phase, respectively was introduced by Weiss et al. ${ }^{17}$ and Kabalnov ${ }^{16}$. The occurrence of enhanced Ostwald ripening over time is favoured by the increased solubility of oil in the emulsifier aggregates.

In order to describe the experimental data in its entirety, a combination of both mechanisms is considered in fitting the data. Considering the lower droplet mode, within uncertainty the mean droplet size does not change up to $\sim 70$ days. Beyond 70 days, the data are best fit by equation 5.3, i.e., the standard Ostwald ripening mechanism explains the oil droplet growth (see fit in Fig. 5.18 (a), $r \propto t^{3.04}$ ). In contrast, for the higher droplet mode, oil 
droplet growth is evident after only $\sim 43$ days after which the mechanism of destabilisation followed is that of enhanced Ostwald ripening (equation 5.4, see fit in Fig. 5.18 (b), $r \propto t^{1.91}$ ). Coalescence $^{19-21}$ is another possible mechanism that leads to the growth of the oil droplets via direct contact. The occurrence of coalescence is expected to be a $\frac{1}{r^{2}}$ dependence $^{22,23}$. Such dependence was absent for our data. Thus, a combination of the two variations of Ostwald ripening most closely describes the measured change in the droplet size distribution as a function of time for this emulsion.

The stability of soybean oil/Na-caseinate/water emulsions from a macroscopic perspective can be attributed specifically to the interactions between soybean oil and the Nacaseinate that control the properties of the interface, i.e., Gaussian curvature, interfacial tension and Gibbs elasticity. According to Bancroft's rule ${ }^{24}$, as Na-caseinate is more soluble in water, the emulsions formed tend to be of the oil-in-water type. Negative Gaussian curvature $^{25}$ does not exist but rather, only positive Gaussian curvature microstructures were formed in this system. This is hinted at by the dependence of the data presented in Fig. 5.18 which corresponds to flocculation (i.e., Ostwald ripening) and coalescence is not observed. Therefore, the interfacial tension and the Gibbs elasticity are the more important interfacial characteristics. Na-caseinate is a strong emulsifier which produces an interface with high interfacial tension and elasticity, this combined with the greater penetration by the highly branched soybean oil (triglyceride), results in a considerably more rigid interfacial domain ${ }^{26}$, ${ }^{27}$ and a more stable emulsion. That is, the interfacial domain is not easily manipulated.

\subsubsection{PGSTE-NMR}

The following is an analysis of the PGSTE-NMR data. For the measurements of the water diffusion coefficients, the water molecules that are firmly attached to the protein molecules do not contribute to the diffusion of water. This is the so-called 'direct hydration effect'. Since diffusion measurements based on the NMR method make no distinction between 'bound' and 'free' water molecules, the experimentally measured diffusion coefficient of the total water content in protein solutions is the superposition of the obstruction effect and the direct hydration effect. In drawing this conclusion, we note that the distance diffused by water molecules according to equation 3.1 over the observation time, $\Delta$ of $20 \mathrm{~ms}$ is $\sim 6$ to 7 $\mu \mathrm{m}$. It should be noted that while the interdroplet spacing in these concentrated emulsions is 
on the order of $200 \mathrm{~nm}$, this is significantly larger than the molecular size of water. Hence, unrestricted diffusion of water is always measured.

In Table 5.1 and Fig. 5.19 are given the calculated (using equation 3.2) and experimental obstruction factors, based on the water diffusion coefficients. Note that the measured density for soybean oil is $0.850 \mathrm{~g} \mathrm{~cm}^{-3}$. Comparing the theoretical and experimental values for the obstruction factor at different $\mathrm{Na}$-caseinate concentrations reveals that there is a general decline as soybean oil concentration was increased from 50 to $60 \mathrm{wt} \%$. The obstruction factors for $3 \mathrm{wt} \% \mathrm{Na}$-caseinate emulsions deviate from the theoretical values to a significantly greater extent than for the $1 \mathrm{wt} \%$ and $2 \mathrm{wt} \% \mathrm{Na}$-caseinate systems. For all samples, the experimental values decreased more rapidly than the theoretical values. The large drop in the measured diffusion coefficients (Fig. 5.5) and the calculated obstruction factors of $3 \mathrm{wt} \% \mathrm{Na}$-caseinate emulsions as compared to the $1 \mathrm{wt} \%$ and $2 \mathrm{wt} \% \mathrm{Na}$-caseinate systems signifies that a significant change in the microstructure which is also manifested in the variation of macroscopic stability (Chapter 4) and evident from the cryo-SEM data (see Fig. 5.17), has occurred. That is, from Chapter 4 we see that emulsions in domain III are the least stable due to the occurrence of extensive depletion flocculation. Depletion flocculation brought about by the excess of protein in the continuous phase ${ }^{28,29}$ triggers the formation of an interdroplet network seen in the cryo-SEM micrograph (Fig. 5.17 (c)). This affects the diffusion pathway of water. Again, the tortuosity effect ${ }^{30,31}$ (as discussed in Chapter 3) induced by the impenetrable oil droplets ${ }^{32}$ must be taken into consideration in understanding the reduction of water diffusion. Indeed, Jönsson et al. showed that this effect is sensitive to droplet shape; that is it is greater for prolate and oblate droplets ${ }^{33}$.

Table 5.1: Theoretical and experimental values of the obstruction factor in the soybean oil/Nacaseinate/water system

\begin{tabular}{ccccc}
\hline \multirow{2}{*}{$\begin{array}{c}\text { Soybean oil conc. } \\
(\mathrm{wt} \%)\end{array}$} & $O_{\text {theo }}$ & \multicolumn{3}{c}{$O_{\exp }$} \\
\cline { 3 - 5 } & & 0.60 & 2 & 3 \\
\cline { 3 - 5 } & 0.79 & 0.56 & 0.56 & 0.45 \\
50 & 0.78 & 0.53 & 0.54 & 0.45 \\
54 & 0.78 & 0.53 & 0.52 & 0.43 \\
56 & 0.77 & 0.51 & 0.49 & 0.39 \\
58 & 0.76 & 0.51 & 0.47 & \\
60 & 0.76 & & 0.46 & \\
\hline
\end{tabular}




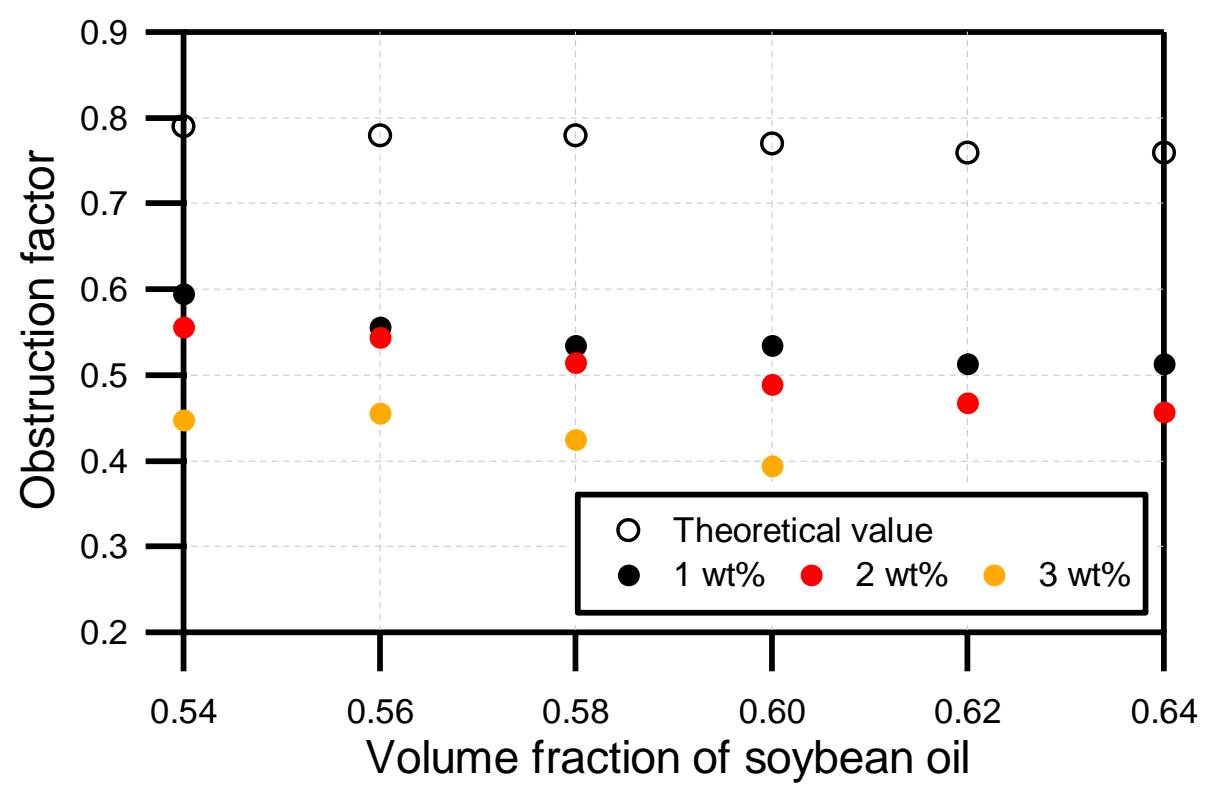

Figure 5.19: Theoretical and experimental obstruction factors for freshly prepared soybean oil/1, 2 and $3 \mathrm{wt} \%$ Na-caseinate/water emulsions.

In fact, considering the water diffusion data in Fig. 5.6, three domains are traversed for $\mathrm{Na}$-caseinate concentrations in the range of 1 to $3 \mathrm{wt} \%$ (domains I, II and III). On increasing Na-caseinate concentration, the water diffusion coefficient remains constant in domain I before decreasing on entering domain II, the second transition occurring at $\sim 2.3 \mathrm{wt} \% \mathrm{Na}-$ caseinate while not as dramatic as the first as can be seen a slight shift. Calculation of the experimental and theoretical obstruction factors based on the water diffusion data also highlight that this second transition is weakly evident, with the deviation of the experimental obstruction factor being enhanced on entering domain III. An approximately $40 \%$ deviation occurs throughout domain $\mathrm{I}$, this is increased by only $4 \%$ for a $0.5 \mathrm{wt} \%$ increase in $\mathrm{Na}$ caseinate concentration within domain II while the same concentration change in domain III results in an increase of over $5 \%$.

Taking into consideration all possible forms of oil diffusion that may have occurred in the system, that is, the unrestricted and restricted diffusion of the oil in the oil droplets and the unrestricted and restricted diffusion of the oil droplets themselves, the oil diffusion data presented in Fig. 5.8, Fig. 5.9 and Fig. 5.10 may be rationalised. The different motions of oil droplets are mainly attributed to the nature of the oil, the droplet sizes, the polydispersity of the emulsion, the concentration of the dispersed phase and the experimental length scale, i.e., the observation time, $\Delta$. 
In recent years, the diffusion phenomena of the dispersed phase in polydisperse emulsions have been investigated via PGSTE-NMR ${ }^{34-36}$. PGSTE-NMR has been reported as a useful characterisation technique in identifying the microstructures of a system ${ }^{37}$. Lindman et al. ${ }^{38}$ and Xu et al. ${ }^{39}$, for example, have investigated a quaternary microemulsion by probing the diffusion coefficients of continuous and dispersed phases; hence enabling a classification into oil-in-water, water-in-oil or bicontinuous structures.

By manipulating the time between the gradient pulses, $\Delta$, both restricted r $^{40-45}$ and unrestricted $^{46}$ diffusion may be monitored. However, because of the high polydispersity of the emulsion droplet size distribution, a superposition of different motions, e.g., restricted diffusion in the oil droplets and of the oil droplets, is possible for each value of $\Delta$ used $^{47-49}$. Hence, the different oil droplet motions coupled with emulsion polydispersity means that a complete droplet size distribution cannot be directly obtained from the diffusion data. Nevertheless, portions of the droplet size distribution may be probed. Therefore, in order to understand our results we will discuss each oil diffusion peak separately and correlate its origin with possible types of diffusion. The discussion on the soybean oil system on varying $\Delta$ for a given composition will then be used as the basis for analysing the diffusion data obtained for different oil and Na-caseinate compositions as well as for different compositions in the palm oil (Chapter 7) and tetradecane (Chapter 8) systems. We will explain the observed diffusion coefficient distribution using the $50 \mathrm{wt} \%$ soybean oil/2 $\mathrm{wt} \% \mathrm{Na}$ caseinate $/ 48 \mathrm{wt} \%$ water emulsion measured at $\Delta=200 \mathrm{~ms}$ as an example (see Fig. 5.8).

The unrestricted diffusion coefficient of soybean oil is on the order of $10^{-10} \mathrm{~m}^{2} \mathrm{~s}^{-1}$. This diffusion is faster than the fastest oil diffusion measured $\left(10^{-11} \mathrm{~m}^{2} \mathrm{~s}^{-1}\right.$, see Fig. 5.8). As such, it does not contribute to the measured diffusion coefficient distribution.

Diffusion peak 1. The dilution experiments (see Fig. 5.8 and Fig. 5.9) confirm that peak 1 in Fig. 5.8 is attributed to restricted diffusion of the oil droplets. As such, the mean square displacement of the oil droplets may be determined from which the smallest average spacing of the oil droplets can be determined. Using equation 3.1, the calculated mean square displacements at different observation times are presented in Fig. 5.20.

For peak 1, the calculated mean square displacement for $\Delta$ from 200 to $800 \mathrm{~ms}$ (the available time range) is $\sim 0.2 \mu \mathrm{m}$ (see Fig. 5.20). This distance is determined to be the average shortest distance between oil droplets in the emulsion. Therefore, any oil droplets of this size or larger may be restricted by the presence of the other oil droplets. That is, depending on the observation time, $\Delta$, only oil droplets with a diameter less than the mean 
distance between the oil droplets will undergo unrestricted diffusion. However, as $\Delta$ increases even these oil droplets will become restricted. As is known, large oil droplets move more slowly than small oil droplets, hence, the slowest oil diffusion peak (peak 1) in Fig. 5.8 is due to the motion of the largest oil droplets in the distribution, i.e., the higher droplet mode of the bimodal droplet size distribution, oil droplets larger than $0.2 \mu \mathrm{m}$ (see Fig. 5.1) undergoing restricted diffusion.

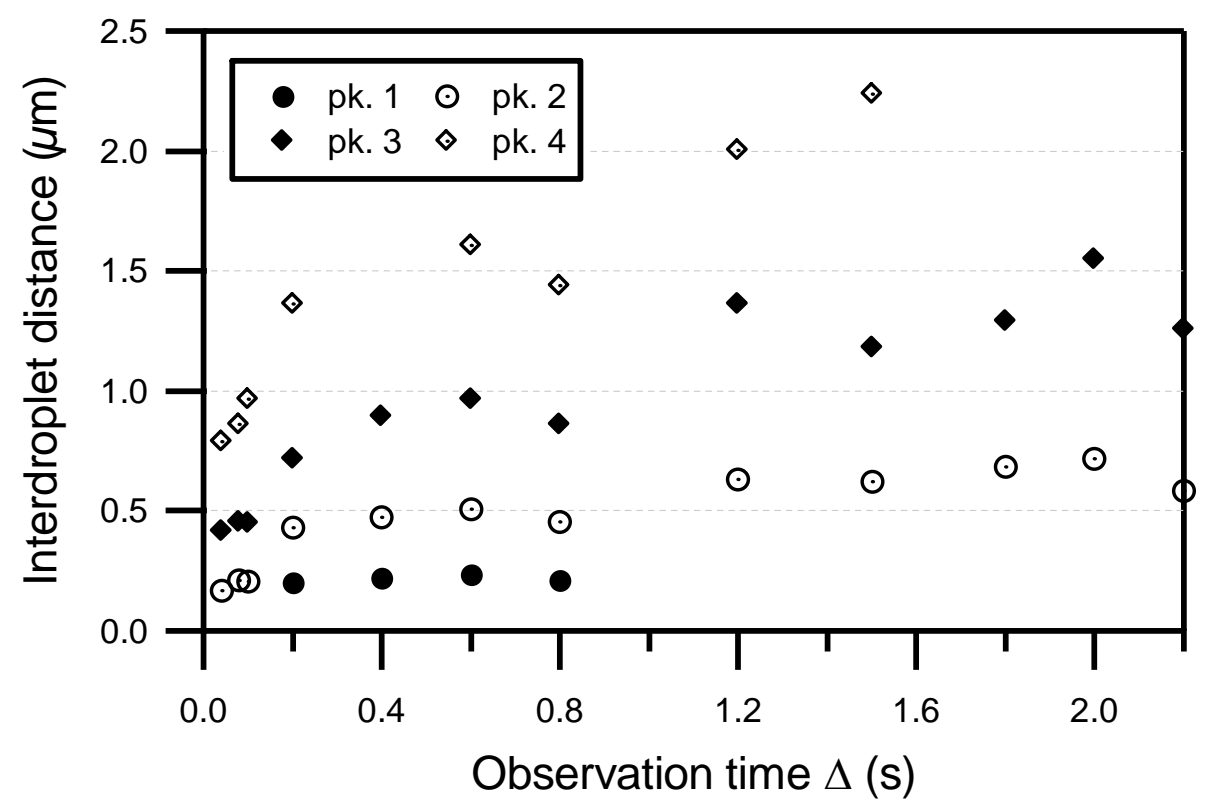

Figure 5.20: The calculated mean square displacement as a function of increasing observation time for three different diffusion coefficients and for diffusion coefficients of the four different oil diffusion peaks shown in Fig. 5.8, respectively.

If the restriction of the oil droplets is caused by the spacing of the oil droplets, i.e., the mean distance between the oil droplets, one would expect that at a fixed Na-caseinate concentration by incrementing the soybean oil concentration, the relative intensity of the oil diffusion peak that is due to pure restricted diffusion should increase, i.e., restricted diffusion between oil droplets will be more pronounced, because of the already enhanced packing of the oil droplets. Upon increasing soybean oil concentration, without concomitantly changing the size of the oil droplets, the number of oil droplets present in the sample must increase. The oil droplets become more closely associated with each other. This was indeed found to be the case in Fig. 5.11 (b), since the oil droplets will encounter each other on average more frequently in highly concentrated samples. 
Diffusion peak 2. For peak 2, considering first the $D$ vs. $\Delta$ plot in Fig. 5.10. The diffusion coefficient obtained up to $\Delta=800 \mathrm{~ms}$ is in a decreasing trend indicating some kind of restricted diffusion. From Fig. 5.20, the calculated mean square displacement (or mean distance between the oil droplets) at the observation time, $\Delta$ of $200 \mathrm{~ms}$ is $0.43 \mu \mathrm{m}$. Peak 2 in Fig. 5.8 thus has a contribution due to restricted diffusion of oil droplets in the higher droplet mode of the bimodal droplet size distribution (see Fig. 5.1). Upon dilution, the signal contribution from this type of diffusion is lost. This leads to a shift in the position of the diffusion peak 2 to faster diffusion coefficients with the only contribution to the peak being unrestricted diffusion of the oil droplets in the higher droplet mode of the bimodal droplet size distribution and restricted diffusion of the oil molecules in the oil droplets.

Considering the possibility of restricted diffusion of the oil molecules in the oil droplets, the mean square displacement for this type of diffusion is described using the following relationship

$$
\left\langle Z^{2}\right\rangle^{1 / 2}=\sqrt{\frac{2}{5} r^{2}}
$$

assuming spherical oil droplets of radius $r$. By combining equation 3.1 and 5.5 yields the estimated diffusion coefficient of the oil molecules in the oil droplets as ${ }^{50}$

$$
D=\frac{1}{5}\left(\frac{r^{2}}{\Delta}\right) \text {. }
$$

In Fig. 5.21 is shown the estimated diffusion coefficient of the oil molecules in the oil droplets at $\Delta=200 \mathrm{~ms}$ using five different oil droplet characteristic sizes within the bimodal droplet size distribution of freshly prepared $50 \mathrm{wt} \%$ soybean oil $/ 2 \mathrm{wt} \% \mathrm{Na}$-caseinate $/ 48 \mathrm{wt} \%$ water emulsion. Note that the estimated diffusion coefficients in Fig. 5.21 spanning from $10^{-16}$ to $10^{-14} \mathrm{~m}^{2} \mathrm{~s}^{-1}$ are beyond the limit of measurable diffusion coefficients. Mapping the measured diffusion coefficient, $D$, at $\Delta=200 \mathrm{~ms}$, which is $4.64 \times 10^{-13} \mathrm{~m}^{2} \mathrm{~s}^{-1}$ with the estimated diffusion coefficients in Fig. 5.21 indicates that restricted diffusion of the oil molecules in the oil droplets near the larger diameter tail of the higher droplet mode contributes to peak 2 in Fig. 5.8.

We now consider the $D \cdot \Delta$ vs. $\Delta$ plot in Fig. 5.10. The product of $D \cdot \Delta$ increases with increasing observation time for long $\Delta$, suggesting that apart from restricted diffusion, peak 2 in Fig. 5.8 also has a contribution due to unrestricted diffusion of the oil droplets in the lower droplet mode of the bimodal droplet size distribution. Owing to the fact that the diffusion coefficients measured for peak 2 is a total contribution of different type of diffusions, true 
unrestricted diffusion of the oil droplets in the lower droplet mode (on the order of $10^{-12} \mathrm{~m}^{2}$ $\mathrm{s}^{-1}$ ) will never be measured.

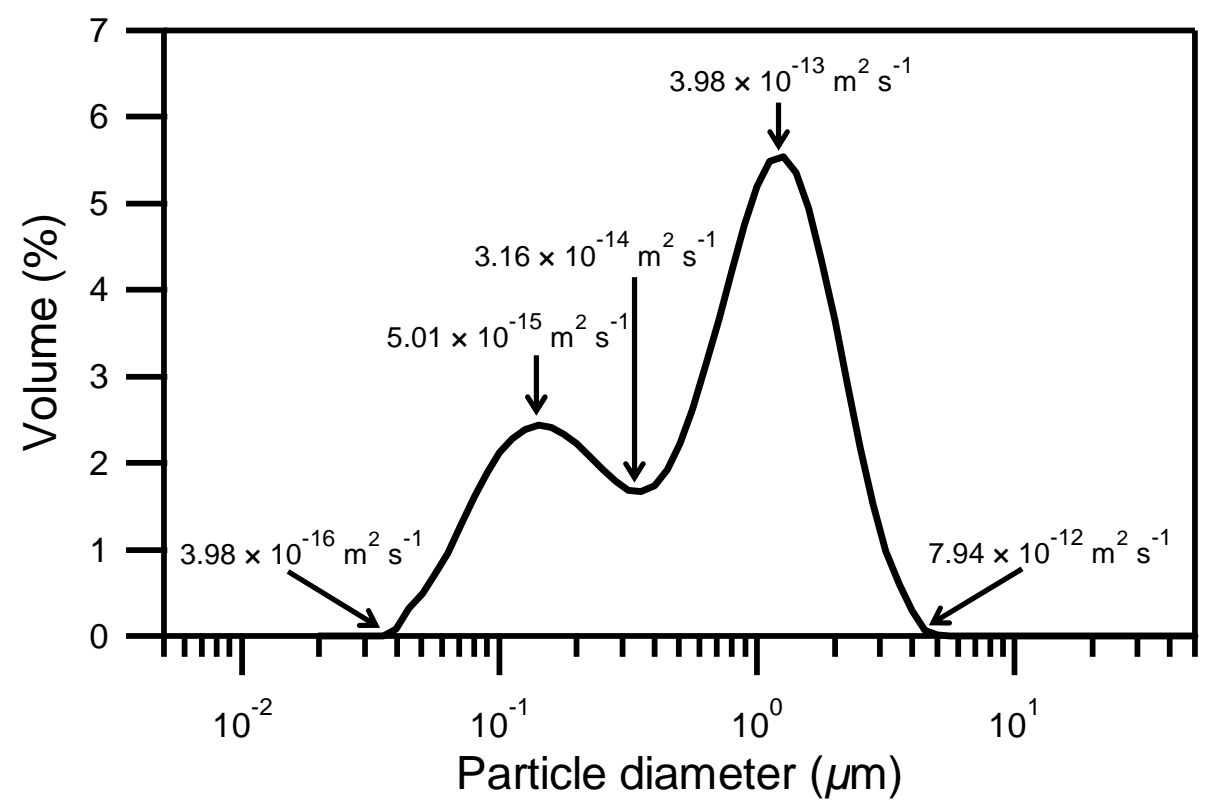

Figure 5.21: Expected diffusion coefficients of the oil molecules in the oil droplets at five different oil droplet characteristic sizes of the bimodal distribution of freshly prepared $50 \mathrm{wt} \%$ soybean oil $/ 2 \mathrm{wt} \% \mathrm{Na}$-caseinate/48 $\mathrm{wt} \%$ water emulsion as indicated by arrows, $\Delta=200 \mathrm{~ms}$.

Diffusion peak 3. For $\Delta \geq 200 \mathrm{~ms}$, the product of $D \cdot \Delta$ for peak 3 in Fig. 5.10 also increases with increasing observation time, revealing that peak 3 in Fig. 5.8 also has a major contribution due to unrestricted diffusion of the oil droplets. Using the Stokes-Einstein relation (equation 3.5) and the measured diffusion coefficient for peak 3 in Fig. $5.8(1.67 \times$ $10^{-12} \mathrm{~m}^{2} \mathrm{~s}^{-1}$ ), it correlates to oil droplets with a diameter of $\sim 0.3 \mu \mathrm{m}$ experiencing unrestricted diffusion. As can be seen from Fig. 5.1, this oil droplet size falls in the lower droplet mode of the bimodal droplet size distribution.

However if we consider the $D$ vs. $\Delta$ plot for peak 3 in Fig. 5.10, $D$ is in a decreasing trend for short $\Delta$, signifying some kind of restricted diffusion. At the observation time, $\Delta$ of $200 \mathrm{~ms}$, the calculated mean square displacement (see Fig. 5.20) is $0.82 \mu \mathrm{m}$ (note that the average shortest distance between oil droplets is $\sim 0.2 \mu \mathrm{m}$ ). This means that predominantly restricted diffusion of the oil droplets with a size $>0.82 \mu \mathrm{m}$ is probed and this contributes to the measured diffusion coefficient for peak 3 in Fig. 5.8. Again, considering the measured diffusion coefficient together with the estimated diffusion coefficients in Fig. 5.21 indicates 
that restricted diffusion of the oil molecules in the oil droplets near the larger diameter tail of the higher droplet mode also contributes to peak 3 in Fig. 5.8.

Diffusion peak 4. The product of $D \cdot \Delta$ over the observation time studied for peak 4 points to unrestricted diffusion being the major contribution to this peak. The $D$ vs. $\Delta$ plot, on the other hand, shows a decreasing trend at short $\Delta$. Hence, there is some minor contribution from restricted diffusion. The calculated mean square displacement using $D$ $\left(7.74 \times 10^{-12} \mathrm{~m}^{2} \mathrm{~s}^{-1}\right)$ obtained at $\Delta=200 \mathrm{~ms}$, i.e., $1.76 \mu \mathrm{m}$ coupled with the disappearance of peak 4 at higher dilution factors (see Fig. 5.8 and Fig. 5.9) suggests that restricted diffusion of the oil droplets is most likely not a contributor to peak 4 in Fig. 5.8 and that the minor restricted diffusion component is due to that of oil molecules in the largest oil droplets. Since these are few in number, upon dilution the probability of detecting this motion decreases.

If unrestricted diffusion of the oil droplets is the major possible contributor to the measured diffusion coefficients of this fastest oil diffusion peak (peak 4), the estimated diffusion coefficients at $\Delta=200 \mathrm{~ms}$ using five different oil droplet characteristic sizes within the bimodal droplet size distribution of freshly prepared $50 \mathrm{wt} \%$ soybean oil $/ 2 \mathrm{wt} \% \mathrm{Na}$ caseinate $/ 48 \mathrm{wt} \%$ water emulsion (from left to right, Fig. 5.21) should span from $\sim 10^{-14}$ to $10^{-11} \mathrm{~m}^{2} \mathrm{~s}^{-1}$. This is clearly the case where peak 4 is solely due to unrestricted diffusion of the smallest oil droplets in the lower droplet mode of the bimodal droplet size distribution (see Fig. 5.21).

Upon dilution, those very small oil droplets become increasingly fewer. Thus, the probability of having very small oil droplets in the NMR tube is very small. Because of the increasingly small contribution they make on a volume basis, the PGSTE-NMR technique becomes blind to the smallest oil droplets in the emulsion upon dilution. This explains why peak 4 disappeared in the diffusion spectrum (Fig. 5.9) at higher emulsion to aqueous phosphate buffer volume ratios.

\subsubsection{Rheology}

$G^{\prime}$ and $G^{\prime \prime}$ are a measure of the energy stored and dissipated as viscous flow in a cycle of oscillation, respectively ${ }^{51}$. Adsorption of protein to the interface results in the formation of a viscoelastic layer ${ }^{52,53}$ and local variation of the interfacial tension contributes to the emulsion's overall elasticity ${ }^{54,55}$. The characteristic viscoelastic response of the dynamic strain sweep shown in Fig. 5.13 exhibits a broad relaxation "plateau" at the applied strain 
amplitude of between $\sim 0.07$ and $10 \%$. This observation is consistent with the findings of others where commercial mayonnaise ${ }^{56,57}$ and pea-protein stabilised concentrated oil-inwater emulsions ${ }^{56}$ were tested.

In concentrated emulsions, small-deformation rheological behaviour is a sensitive probe of the colloidal interactions between adsorbed protein layers on different droplets ${ }^{58}$. In Fig. 5.13, we see that $G^{\prime}$ and $G^{\prime \prime}$ in the linear regime of the emulsions encompassing domain I to III in the phase diagram increase. This reveals that the linear rheological response is thereby dominated by the volume fraction of the dispersed phase and/or the amount of emulsifier present, i.e., the microstructure variability. Higher soybean oil volume fractions and Nacaseinate concentration promote the packing of the oil droplets and hence results in the enhancement of viscoelastic properties of the system. The linear rheological results presented in Fig. 5.15 further show the microstructure of emulsions studied is Na-caseinate concentration dependence. This is supported by the PGSTE-NMR results (Fig. 5.12). The increasing effect of protein concentration on the linear viscoelastic properties of our emulsions is similar to those stabilised with carob protein ${ }^{59}$, gluten and soya proteins ${ }^{60}$. However, the dynamic frequency sweeps of those systems is characterised by a slight frequency dependence for $G^{\prime}$ and a minimum in $G^{\prime \prime}$. The occurrence of this behaviour is related to the formation of physical entanglements among proteins adsorbed at the oil/water interface of the oil droplets which leads to the formation of an elastic structural network ${ }^{61-63}$.

Our results in Fig. 5.15, on the other hand, show that the frequency dependent storage and loss moduli run parallel with each other with $G^{\prime}$ higher than $G^{\prime \prime}$, as previously reported for commercial or model mayonnaise ${ }^{64-66}$ and salad dressing-type emulsions ${ }^{64,67}$. Diftis et al. also attributed such behaviour to the formation of a pseudo-gel network due to entanglements among protein segments adsorbed at the oil/water interface of the neighbouring oil droplet. The slight increase in $G^{\prime}$ and $G^{\prime \prime}$ on incrementing the frequency (i.e., for emulsions containing 2, 3 and 4 wt $\%$ Na-caseinate) further support the gel-like structure predominated in the emulsions and can be understood as follows: the oil droplets are more deformed on increasing frequency, hence they store more energy until the aggregated/flocculated structures, due to droplet-droplet interaction, is disrupted at the highest frequency ${ }^{67}$. Indeed, the formation of gel-like structure demonstrated in Fig. 5.15 correlates well with the self-assembly of the Nacaseinate to form a three-dimensional network as exemplified by the data shown in Chapter 3 and the cryo-SEM micrographs shown in Fig. 5.17. 
$G^{\prime}$ and $G^{\prime \prime}$ increase up to about two orders of magnitude higher for emulsions prepared with $4 \mathrm{wt} \% \mathrm{Na}$-caseinate as compared with those consist of $1 \mathrm{wt} \% \mathrm{Na}$-caseinate, revealing a more elastic-like behaviour due to the formation of a three-dimensional network within the continuous phase in addition to the compression and packing of the oil droplets. Nonetheless, emulsions prepared with $>1 \mathrm{wt} \% \mathrm{Na}$-caseinate are in an undisturbed ordered state as $G^{\prime}$ and $G^{\prime \prime}$ are constant. During the entire range of applied frequency, neighbouring oil droplets push against each other but the osmotic pressure and other forces are in equilibrium.

The basic parameters determining the non-linear rheology of an emulsion are (i) continuous phase rheology (which is not investigated here); (ii) nature of the dispersed phase, size distribution, deformability, internal viscosity, concentration and; (iii) nature of dropletdroplet interaction ${ }^{68}$. From a simplistic point of view, steady state shearing at any given rate induces both rotational and elongational flow of the emulsions ${ }^{69}$. The idealised deformation of the oil droplets at sufficiently high shear rates is that the oil droplets experience a prolate deformation which then becomes dumbbell shaped and eventually breaks up to undergo laminar flow ${ }^{70}$. While shear flow causes distortion and disruption to the oil droplets and temporarily formed structures, Brownian motion which dominates at low shear rates, on the other hand, randomising the movement of oil droplets promoting aggregation and coalescence. In reality, as we shall show in the following chapter, the steady shear rheology of this soybean oil/Na-caseinate/water system is much more complicated.

\subsubsection{Cryo-SEM}

Recently, cryo-SEM has been widely used to determine the microstructure of emulsions. In the cosmetic industry, for example, this methodology contributes both to the understanding of the relation between microstructures and the design of novel oil-in-water type emulsion products $^{71}$. Etching of the continuous phase was incorporated with this technique to identify phase inversion from water/oil (w/o) to o/w occurring during the cream manufacture in addition to monitoring the cream stability during long-term storage ${ }^{72}$. John et al. have shown, using soybean oil and modified versions thereof, that cryo-SEM enables elucidation of the oil-surfactant-water interaction in the emulsion ${ }^{73}$. It was found from the electron micrographs that the phase behaviour of emulsions was dependent on the nature (ionic or non-ionic) and the concentration of surfactant used. Modified soybean oil required 
comparatively increased amounts of surfactant than the regular soybean oil to obtain a stable emulsion.

The electron micrographs shown in Fig. 5.16 and Fig. 5.17 present the difference in microstructure for emulsions in different domains in the phase diagram of the soybean oil system. Emulsion microstructure is primarily defined by the extent of oil droplet surface coverage by the Na-caseinate, i.e., the structure of the interfacial thin film and the state of Nacaseinate self-assembly. Bridging flocculation due to incomplete coverage of the oil droplet surface $^{74}$ which occurs at low protein/oil ratios is evident in Fig. 5.17 (a). The oil droplets are seen to join with neighbouring oil droplets leading to the loss in oil droplet identity. In contrast, discrete oil droplets can be easily visualised in Fig. 5.17 (b) because the oil droplets surfaces are sufficiently covered with the emulsifier at such Na-caseinate concentration.

Further increasing the Na-caseinate concentration induces depletion flocculation to occur. An excess of protein exists in the aqueous phase. The Na-caseinate molecules and aggregates are expelled from the region of closest proximity between the oil droplets. The excess Na-caseinate begins to interact, the structure of the continuous phase is altered and initial network formation is seen (Fig. 5.17 (c), (d) and (e)).

Finally, the caseinate molecules self assemble throughout the aqueous continuous phase, achieving a three-dimensional network (Fig. 5.17 (f)). The microstructure consists of individual oil droplets with well-resolved interfaces in an aqueous protein network, having a pore structure below $100 \mathrm{~nm}$. The oil droplets are able to rearrange only slowly and are essentially trapped, which concomitantly increases the life time of the emulsions. The formation of a three-dimensional protein network is in good agreement with the data presented in Chapter 3.

\subsection{Conclusions}

All of the emulsions formed in domains I, II, III and IV are oil-in-water emulsions. They exhibit similar droplet size distributions upon preparation. Evolution of the droplet size distribution as a function of time showed an increase in average droplet size. The destabilisation mechanism for the lower and higher droplet modes are standard and enhanced Ostwald ripening processes, respectively. 
From the PGSTE-NMR results, at a fixed Na-caseinate concentration, the diffusion coefficient of water is a decreasing function of increasing soybean oil concentration. At a fixed soybean oil concentration, the water diffusion coefficient remained constant before displaying a decrease at $\sim 1.5 \mathrm{wt} \%$ of Na-caseinate corresponding to the transition between domain I and II in the phase diagram. An indication of the transition from domain II to III was observed. Both transitions were clearly seen in the oil diffusion data. At a fixed Nacaseinate concentration, the relative intensity of the slowest oil diffusion peak, due to pure restricted diffusion between oil droplets, increased on incrementing soybean oil concentration; at a fixed soybean oil concentration, it remained unchanged, followed by an increase at $\sim 1.5 \mathrm{wt} \%$ of $\mathrm{Na}$-caseinate (domain I to II transition) before returning to an almost constant value at approximately $2.25 \mathrm{wt} \%$ of Na-caseinate (domain II to III transition). For the soybean oil diffusion, the extracted distribution of diffusion coefficients was a mixture of three contributions, unrestricted and restricted diffusion of the oil droplets themselves and restricted diffusion of the oil molecules in the oil droplets.

Five separate microstructures were evidenced from cryo-SEM: bridging flocculation, individual oil droplets in a water continuum, depletion flocculation, interdroplet network formation and a three-dimensional protein network.

At a fixed Na-caseinate concentration, the emulsions showed identical viscoelastic properties with a weak power law dependence over the three decades of frequency investigated. The emulsions are predominantly solid-like. At a fixed soybean oil concentration, $G^{\prime}$ and $G^{\prime \prime}$ increased on increasing Na-caseinate concentration. 


\subsection{References}

1. P. T. Callaghan, S. Godefroy and B. N. Ryland, Magn. Reson. Imaging 21, 243-248 (2003).

2. S. W. Provencher, Comput. Phys. Commun. 27, 229-242 (1982).

3. D. A. Reinelt and A. M. Kraynik, J. Colloid Interface Sci. 159, 460-470 (1993).

4. D. M. A. Buzza and M. E. Cates, Langmuir 10, 4503-4508 (1994).

5. J. Sjöblom, Emulsion and emulsion stability, 2nd ed. (CRC Press, New York, 2006).

6. M. B. J. Meinders and T. van Vliet, Adv. Colloid Interface Sci. 108-109, 119-126 (2004).

7. R. Aveyard, B. P. Binks, J. Esquena, P. D. I. Fletcher, P. Bault and P. Villa, Langmuir 18, 3487-3494 (2002).

8. J. Weiss, N. Herrmann and D. J. McClements, Langmuir 15, 6652-6657 (1999).

9. J. Soma and K. D. Papadopoulos, J. Colloid Interface Sci. 181, 225-231 (1996).

10. I. M. Lifshitz and V. V. Slezov, J. Phys. Chem. Solids 19, 35-50 (1961).

11. L. Taisne and B. Cabane, Langmuir 14, 4744-4752 (1998).

12. C. Wagner, Elektochem. 65, 581-591 (1961).

13. B. P. Binks, W.-G. Cho, P. D. I. Fletcher and D. N. Petsev, Langmuir 16, 1025-1034 (2000).

14. Y. D. Smet, L. Deriemaeker and R. Finsy, Langmuir 15, 6745-6754 (1999).

15. D. J. McClements and S. R. Dungan, J. Phys. Chem. 97, 7304-7308 (1993).

16. A. S. Kabalnov, Langmuir 10, 680-684 (1994).

17. J. Weiss, C. Canceliere and D. J. McClements, Langmuir 16, 6833-6838 (2000).

18. J. Weiss, J. N. Coupland, D. Brathwaite and D. J. McClements, Colloids Surfs. A 121, 53-60 (1997).

19. F. Leal-Calderon and P. Poulin, Curr. Opin. Colloid Interface Sci. 4, 223-230 (1999).

20. D. T. Wasan, A. D. Nikolov and F. Aimetti, Adv. Colloid Interface Sci. 108-109, $187-$ 195 (2004).

21. I. B. Ivanov, K. D. Danov and P. A. Kralchevsky, Colloids Surfs. A 152, 161-182 (1999).

22. B. Deminière, in Modern aspect of emulsion science, edited by B. P. Binks (The Royal Society of Chemistry, Cambridge, 1998), pp. 261-291. 
23. P. Izquierdo, J. Esquena, T. F. Tadros, J. C. Dederen, M. J. Garcia, N. Azemar and C. Solans, Langmuir 18, 26-30 (2002).

24. W. D. Bancroft, J. Phys. Chem. 17, 501-519 (1913).

25. M. Kahlweit and R. Strey, Angew. Chem., Int. Ed. Engl. 24, 654-668 (1985).

26. B. P. Binks, H. Kellay and J. Meunier, Europhys. Lett. 16, 53-58 (1991).

27. S. Chaieb, B. P. Binks and J. Meunier, J. Phys. II France 5, 1289-1296 (1995).

28. E. Dickinson and M. Golding, Food Hydrocolloids 11, 13-18 (1997).

29. S. J. Radford and E. Dickinson, Colloids Surfs. A 238, 71-76 (2004).

30. H. Jóhannesson and B. Halle, J. Chem. Phys. 104, 6807-6817 (1996).

31. L. Coppola, C. L. Mesa, G. A. Ranieri and M. Terenzi, J. Chem. Phys. 98, 5087-5090 (1993).

32. P. G. Nilsson and B. Lindman, J. Phys. Chem. 87, 4756-4761 (1983).

33. B. Jönsson, H. Wennerstrom, P. G. Nilsson and P. Linse, Colloid Polym. Sci. 264, 7788 (1986).

34. L. Ambrosone, A. Ceglie and G. Colafemmina, J. Chem. Phys. 107, 10756-10763 (1997).

35. L. Ambrosone, A. Ceglie, G. Colafemmina and G. Palazzo, Langmuir 15, 6775-6780 (1999).

36. L. Ambrosone, A. Ceglie, G. Colafemmina and G. Palazzo, J. Chem. Phys. 110, 797804 (1999).

37. I. Lönqvist, A. Khan and O. Söderman, J. Colloid Interface Sci. 144, 401-411 (1991).

38. B. Lindman and P. Stilbs, in Surfactant science series, edited by H. L. Rosana and M. Clausse (Marcel Dekker, New York, 1987), Vol. 24, pp. 125-168.

39. J. Xu, G.-Z. Li, Z.-Q. Zhang, G.-W. Zhou and K.-J. Ji, Colloids Surfs. A 191, 269-278 (2001).

40. B. Balinov, P. Linse and O. Söderman, J. Colloid Interface Sci. 182, 539-548 (1996).

41. C. Malmborg, D. Topgaard and O. Söderman, J. Colloid Interface Sci. 263, 270-276 (2003).

42. D. Topgaard, C. Malmborg and O. Söderman, J. Magn. Res. 156, 195-201 (2002).

43. P. T. Callaghan, A. Coy, T. P. J. Halpin, D. MacDowan, J. K. Packer and F. O. Zelaya, J. Chem. Phys. 97, 651-662 (1992).

44. R. W. Mair, G. P. Wong, D. Hoffmann, M. D. Hürlimann, S. Patz, L. M. Schwartz and R. L. Walsworth, Phys. Rev. Lett. 83, 3324-3327 (1999). 
45. P. P. Mitra, P. N. Sen and L. M. Schwartz, Phys. Rev. B 47, 8565-8574 (1993).

46. L. Ambrosone, S. Murgia, G. Cinelli, M. Monduzzi and A. Ceglie, J. Phys. Chem. B 108, 18472-18475 (2004).

47. T. Garasanin, T. Cosgrove, L. Marteaux, A. Kretschmer, A. Goodwin and K. Zick, Langmuir 18, 10298-10304 (2002).

48. K. G. Hollingworth, A. J. Sederman, C. Buckley, L. F. Gladden and M. L. Johns, J. Colloid Interface Sci. 274, 244-250 (2004).

49. E.-H. Liu, H. Egger and K. M. McGrath, J. Phys. Chem. B 109, 13332-13341 (2005).

50. P. T. Callaghan, Principles of Nuclear Magnetic Resonance Microscopy. (Oxford University Press Inc., New York, 1991).

51. T. Tadros, Adv. Colloids Interface Sci. 108-109, 227-258 (2004).

52. J. Benjamins, Wageningen University, 2000.

53. P. Walstra and A. L. de Roos, Foods Revs. Int. 9, 503-525 (1993).

54. D. M. A. Buzza, C.-Y. D. Lu and M. E. Cates, J. Phys. II France 5, $37-52$ (1995).

55. Y. Hemar, R. Hocquart and F. Lequeux, J. Phys. II France 5, 1567-1576 (1995).

56. C. Bower, C. Gallegos, M. R. Mackley and J. M. Madiedo, Rheol. Acta 38, 145-149 (1999).

57. C. Gallegos, M. Berjano and L. Choplin, J. Rheol. 36, 465-478 (1992).

58. E. Dickinson, in Modern aspects of emulsion science, edited by B. P. Binks (Royal Society of Chemistry, Cambridge, 1998), pp. 145.

59. C. Bengoechea, M. C. Puppo, A. Romero, F. Cordobés and A. Guerrero, J. Food Eng. 87, 124-135 (2008).

60. C. Bengoechea, F. Cordobés and A. Guerrero, Rheol. Acta 46, 1-9 (2006).

61. J. M. Franco, A. Guerrero and C. Gallegos, Rheol. Acta 34, 513-524 (1995).

62. A. Guerrero, P. Partal and C. Gallegos, J. Rheol. 42, 1375-1388 (1998).

63. D. J. McClements, Food emulsions: principles, practice, and techniques. (CRC Press, Boca Raton, 1999).

64. L. B. Famuso, M. Corredig and C. C. Akoh, J. American Oil Chemists' Soc. 78, 771$774(2001)$.

65. J. M. Franco, P. Partal, D. Ruiz-Marquez, B. Conde and C. Gallegos, J. American Oil Chemists' Soc. 77, 975-983 (2000).

66. J. E. Moros, J. M. Franco and C. Gallegos, J. American Oil Chemists' Soc. 79, 837$843(2002)$. 
67. S. L. Turgeon, C. Sanchez, S. F. Gauthier and P. Paquin, Int. Dairy J. 6, 645-658 (1996).

68. H. A. Barnes, Colloids Surfs. A 91, 89-95 (1994).

69. P. Becher, Emulsion: theory and practice, 2nd ed. (Reinhold, New York, 1966).

70. P. Becher, Encyclopedia of emulsion technology. (Marcel Dekker, Inc., New York, 1983).

71. M. Yamashita, K. Kameyama, R. Kobayashi, A. Asahina, S. Aita and K. Ogura, J. Electron Microscopy 45, 461-462 (1996).

72. S. J. Holland and J. K. Warrack, Int. J. Pharmaceutics 65, 225-234 (1990).

73. J. John, M. Bhattacharya and P. C. Raynor, Colloids Surfs. A 237, 141-150 (2004).

74. E. Dickinson, M. Golding and J. W. P. Malcolm, J. Colloid Interface Sci. 185, 515529 (1997). 


\section{Chapter 6}

\section{Shear Banding}

\subsection{Introduction}

Emulsions are an example of complex fluids whose rheological behaviours are strongly dependent on the interaction of the oil droplets ${ }^{1}$. Factors that determine the flow pattern of emulsions include the volume fraction of the dispersed phase and the size of the droplets ${ }^{2,3}$.

Dilute emulsions, with dispersed phase volume fractions generally well below 40 to $45 \%$, most often display either shear-thinning or Newtonian behaviour, with some systems showing shear-thinning at low shear rates followed by Newtonian flow at higher shear rates ${ }^{4-}$ 7; however, the range of shear rates explored is often very limited in its extent and as such the full behaviour of many systems is not well known. In addition to the volume fraction and droplet size, the extent of flocculation of the dispersed phase also strongly influences flow behaviour. Dickinson et al. have reported that non-flocculated dilute emulsions exhibit Newtonian flow, while in the flocculated state the emulsions display shear-thinning behaviour $^{4,8}$.

In contrast to dilute emulsions, linear and non-linear rheological properties of concentrated emulsions remain poorly understood. In addition, these systems are commonly examined only over a narrow range of shear rates ${ }^{9-13}$. Concentrated emulsions share a remarkable number of similarities with other concentrated soft materials such as gels, foams, pastes and suspensions, which are common shear-thinning yield stress fluids ${ }^{14,15}$. Binks et al. ${ }^{16}$ and Masalova and Malkin ${ }^{12}$ have shown, using two different concentrated emulsions, that a yield stress may be present. However, in both cases, analysis of the low shear rate region was restricted due to sample characteristics or instrument limitations. Hence definitive characterisation of the yield stress region was not possible.

Recent evidence has shown that concentrated emulsions display rather more complex flow behaviour, including undergoing a transition from solid-like to fluid-like characteristics 
under shear ${ }^{17-24}$. The occurrence of such a transition may depend on the interaction between components $^{25}$ or the confined flows ${ }^{26,27}$ in the system. Concentrated emulsions appear to have flow behaviour more reminiscent of that ascribed to soft glassy materials rather than that typically shown by emulsions.

An example of the flow behaviour exhibited by soft glassy materials is shear banding or gradient banding, first observed by Decruppe et al. and Mair et al. in worm-like micelle solutions $^{28,29}$. This phenomenon is described as the formation of bands within the sample having different shear rates. These bands form normal to the direction of the flow-gradient ${ }^{30}$. The coexistence of bands at a common stress has been suggested to originate from an underlying flow curve with a negative slope ${ }^{31}$. The decreasing shear stress is inherently unstable and steady shear flow cannot persist ${ }^{32}$. Experimentally, the observed shear stress follows a horizontal plateau as a function of shear rate.

The observance of a stress plateau, however, is not direct evidence for a shear-banded state. A stress plateau may also indicate a yield stress. This is particularly true if the flow curve is represented by a stress plateau and a high shear rates branch only, thus making it difficult to distinguish between a yield stress and a shear band. In contrast, the presence of a yield stress cannot be used to explain the occurrence of a stress plateau when both the high shear rates and low shear rates branches bound the plateau ${ }^{23,33}$.

Recently Ovarlez et al. speculated that shear banding should be expected behaviour, rather than a rare occurrence for complex structured materials and/or those exhibiting nonlinear rheological behaviour such as for the system investigated here ${ }^{34}$. Furthermore, based on literature case studies of worm-like micelles, soft-jammed materials and granular pastes they showed that shear banding appears to be the result of a number of different underlying physical phenomena including phase transitions and spontaneous destruction/restructuration. The primary commonality seemingly being that a minimum of two competing physical effects switch dominance as a function of flow. This switch rather than any specific molecular or particle interactions at the nanoscale therefore drives the formation of the banded state. In order to probe the ideas of Ovarlez et al. further it is necessary to explore the ubiquity of shear banding in a range of complex materials and to ascertain for each system the underlying phenomenon driving the observed viscosity bifurcation.

Herein, we report on shear banding in concentrated Na-caseinate emulsions. To gain insight into the relationship between the Na-caseinate and oil composition, which dictates the macroscopic stability of emulsions, and their corresponding mechanical and microscopic 
properties, we performed conventional rheology measurements in a cone-and-plate geometry and examined the shear flow behaviour of concentrated Na-caseinate emulsions using NMR velocimetry.

\subsection{Results}

\subsubsection{Rheology}

The $50 \mathrm{wt} \%$ soybean oil $/ 2 \mathrm{wt} \%$ Na-caseinate $/ 48 \mathrm{wt} \%$ water emulsion will be used to demonstrate our rheological findings.

The data displayed in Fig. 6.1 show the evolutionary stress response of $50 \mathrm{wt} \%$ soybean oil $/ 2 \mathrm{wt} \% \mathrm{Na}$-caseinate $/ 48 \mathrm{wt} \%$ water emulsions as a function of shearing time (1 minute or 15 minutes). For a shearing time of 1 minute the stress plateau, clearly evident in the 15 minutes data, is ill-defined, manifesting itself only as a point of inflexion. No changes in the flow curve occur for shearing times longer than 15 minutes, while between 1 and 15 minutes a continual progression between the two limits arises. As such for shearing times shorter than 15 minutes the response cannot be considered as steady state. Such an evolution of the flow curve with shearing time is common in soft materials and has recently been documented for a colloidal star glass system ${ }^{35}$.

Four rheological responses (marked I to IV in Fig. 6.1) can be distinguished in the upramping sweep for a shearing time of 15 minutes. First, for very low shear rates the apparent stress observed corresponds to successive, very small local deformations of the material (region I). Second, above a critical stress value, the sample exhibits standard shear-thinning behaviour (such behaviour is most commonly observed for dilute emulsions), (region II). Upon further increasing the shear rate, an apparent stress plateau occurs (region III, with limits defined for this sample by $\dot{\gamma}_{1}=0.02 \mathrm{~s}^{-1}$ and $\dot{\gamma}_{2}=0.794 \mathrm{~s}^{-1}$, where $\dot{\gamma}_{1}$ and $\dot{\gamma}_{2}$ are the shear rates at the beginning and the end of the stress plateau, respectively). Here the imposition of different shear rates results in a constant stress being reached. Finally, just after the apparent stress plateau the stress and the shear rate increase together (region IV), this Newtonian region being the result of complete destruction of the sample macrostructure. 


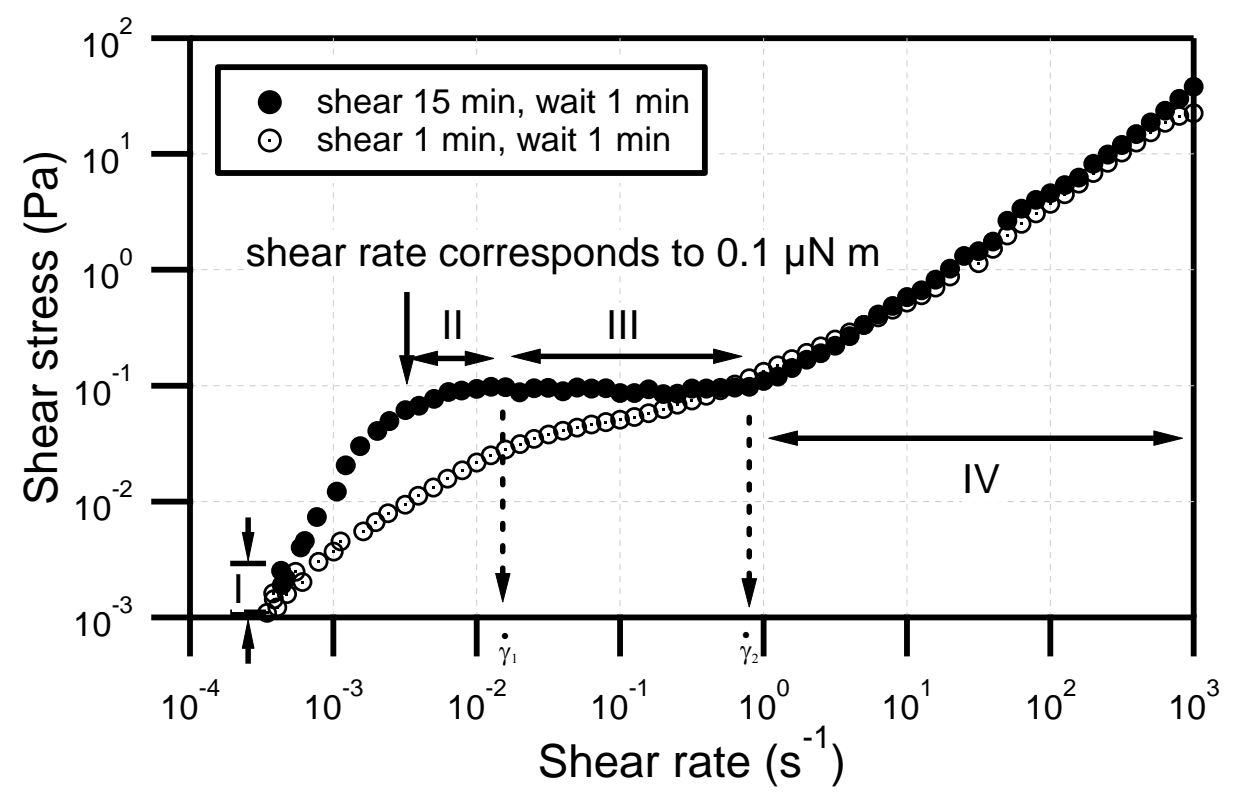

Figure 6.1: Steady state stress as a function of applied shear rate curves for $50 \mathrm{wt} \%$ soybean oil/2 wt $\% \mathrm{Na}-$ caseinate/48 wt\% water emulsion during an up-stress ramping: 15 and 1 min shearing, followed by 1 min rest for each shear rate, from 0.0001 to $1000 \mathrm{~s}^{-1}$. In the $15 \mathrm{~min}$ shearing data, four rheological responses are identified: I apparent yield stress, II shear-thinning with a lower shear rate of $0.00346 \mathrm{~s}^{-1}$ corresponding to the minimum torque the instrument can apply $(0.1 \mu \mathrm{N} \mathrm{m})$, III stress plateau bound by shear rates $\dot{\gamma}_{1}$ and $\dot{\gamma}_{2}$ and IV Newtonian. The data of 1 min shearing show the effect of shearing time on the observed flow curve. The stress plateau is only seen as shearing time is increased resulting in an equilibrium stress response of the sample.

Fig. 6.2 shows the steady state flow curves for $55 \mathrm{wt} \%$ soybean oil/2, 3, 4 and $5 \mathrm{wt} \%$ Na-caseinate/water emulsions at different shearing times. The stresses, corresponding to the four rheological responses as distinguished in Fig. 6.1, are increasing functions of $\mathrm{Na}$ caseinate concentration. $\dot{\gamma}_{1}$ fluctuates around $10^{-2} \mathrm{~s}^{-1}$ and $\dot{\gamma}_{2}$ is on the order of $1 \mathrm{~s}^{-1}$ as extracted from the data shown in Fig. 6.2 (Table 6.1). These limits were obtained by determining the extent of the plateau (defined using the fractional variation of the stress value with respect to its standard deviation with a range of $\pm 6 \%$ ) and taking the corresponding limiting shear rates. These critical shear rates span approximately two orders of magnitude, irrespective of soybean oil or Na-caseinate concentration, and despite the large variation observed in the macroscopic stability and microstructure of the emulsions. No specific trend is seen for shear rate, $\dot{\gamma}_{1}$ or $\dot{\gamma}_{2}$ on varying soybean oil concentration or for $\dot{\gamma}_{1}$ or $\dot{\gamma}_{2}$ on varying Na-caseinate concentration. 


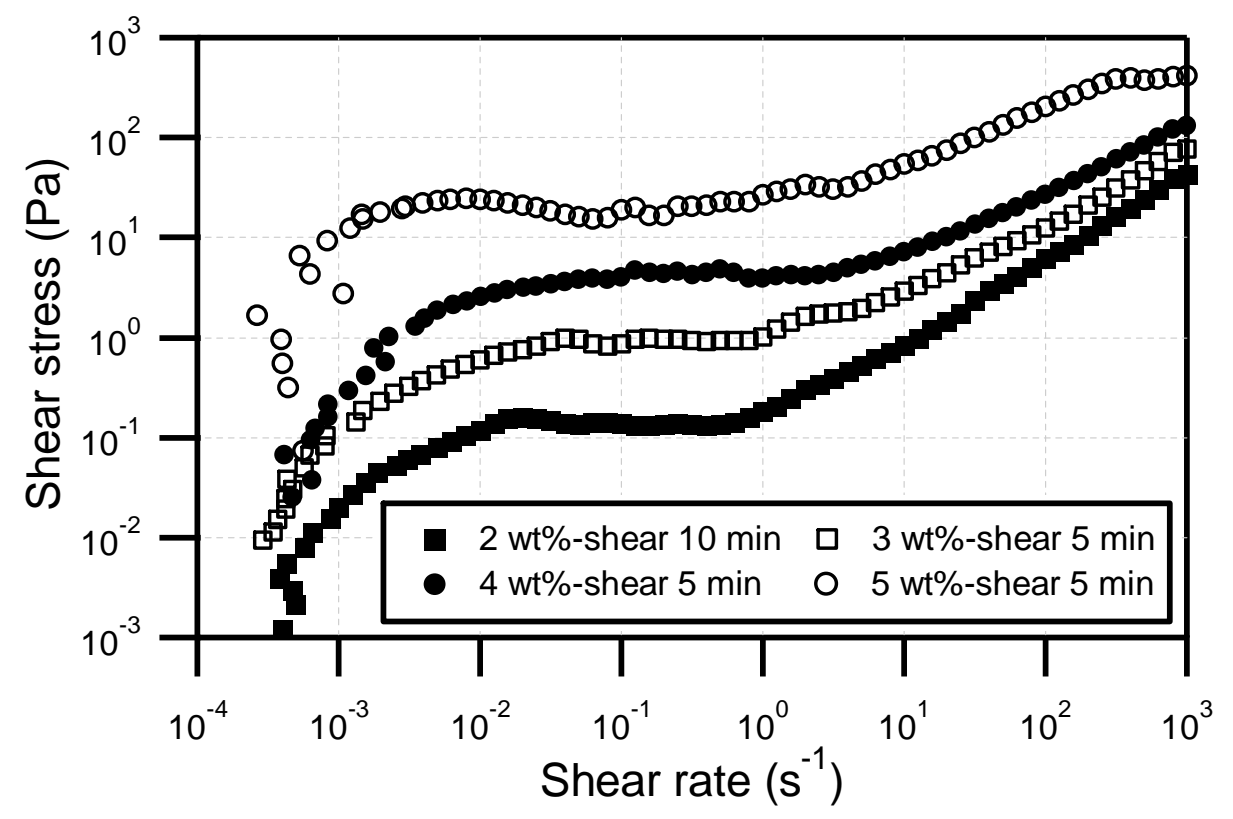

Figure 6.2: Steady state stress vs. shear rate curves for $55 \mathrm{wt} \%$ soybean oil/2, 3, 4 and $5 \mathrm{wt} \% \mathrm{Na}$-caseinate/water emulsions during up-stress ramping: 1 min rest between each shear rate, from 0.0001 to $1000 \mathrm{~s}^{-1}$. Shearing times required to yield a steady state response varied with Na-caseinate concentration and are given in the legend. $\dot{\gamma}_{1}$ and $\dot{\gamma}_{2}$ values (see definition in Fig. 6.1) remain similar and span approximately two orders of magnitude on increasing Na-caseinate concentration.

Table 6.1: $\dot{\gamma}_{1}$ and $\dot{\gamma}_{2}$ values extracted from steady state flow curves for $55 \mathrm{wt} \%$ soybean oil emulsions plotted in Fig. 6.2

\begin{tabular}{ccccc}
\hline Critical shear rate & \multicolumn{5}{c}{ Na-caseinate concentration (wt\%) } \\
$\left(\mathrm{s}^{-1}\right)$ & 2 & 3 & 4 & 5 \\
\hline$\dot{\gamma}_{1}$ & 0.0158 & 0.0316 & 0.0501 & 0.00631 \\
$\dot{\gamma}_{2}$ & 0.631 & 1.00 & 2.51 & 0.794 \\
\hline
\end{tabular}

The steady state flow curves for the corresponding palm oil and tetradecane emulsions were also explored (see Chapters 7 and 8, respectively). The flow curves for the three oil systems were similar and the two critical shear rates defining the plateau region were within experimental uncertainty: $\dot{\gamma}_{1}$ approximately equal to $10^{-2} \mathrm{~s}^{-1}$ and $\dot{\gamma}_{2}$ on the order of $1 \mathrm{~s}^{-1}$.

The shear stress at which the plateau occurs, increases with Na-caseinate concentration. The average shear stress in the plateau as a function of Na-caseinate concentration is essentially an exponential relationship (Fig. 6.3), with a near 100-fold increase in stress for a 2.5-fold increase in Na-caseinate concentration. This monofunctional dependence of the average shear stress of the plateau on Na-caseinate concentration belies the observed underlying phase transitions that occur at $\sim 2.3$ and $\sim 3.3 \mathrm{wt} \%$ for this soybean oil 
concentration, as evidenced by the abrupt changes in emulsion stability and the cryo-SEM data, upon increasing Na-caseinate concentration (see Chapters 4 and 5). For a fixed Nacaseinate concentration, upon varying soybean oil concentration no significant change in the average shear stress value of the plateau was observed.

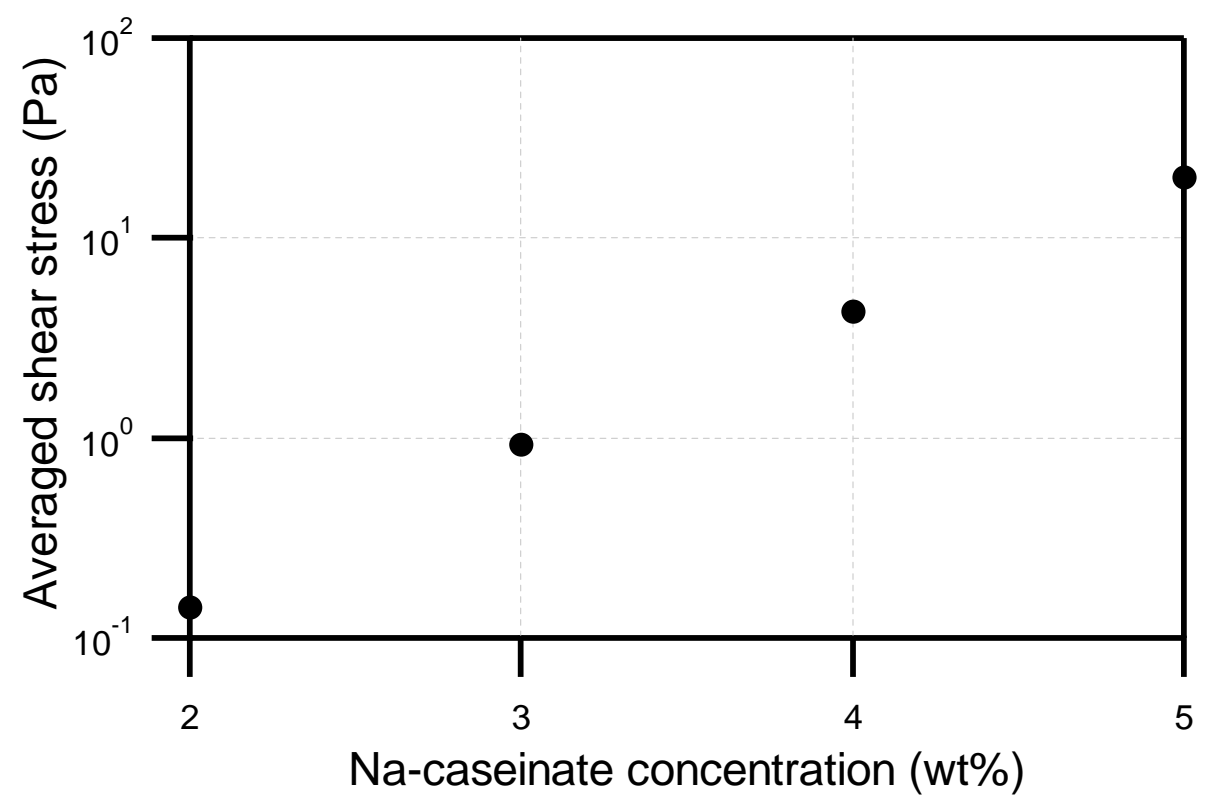

Figure 6.3: Variation of the average shear stress value in the plateau region upon increasing Na-caseinate concentration for $55 \mathrm{wt} \%$ soybean oil/ $\mathrm{Na}$-caseinate/water emulsions.

Fig. 6.4 shows the response of the $50 \mathrm{wt} \%$ soybean oil $/ 2 \mathrm{wt} \%$ Na-caseinate $/ 48 \mathrm{wt} \%$ water sample at different shear rates in the down-ramping sweep. Irrespective of the shearing time, the stress decreases uniformly when moving down along the high shear rates branch. This uniform decrease is followed in all cases by a minimum in the flow curves and a subsequent increase in stress as the shear rate is reduced further.

To further investigate region II in Fig. 6.1 (i.e., the apparent shear-thinning region), creep experiments were performed. In Fig. 6.5 are represented the results of these creep experiments carried out by varying the applied torque within the range of existence of this behaviour ( 0.1 to $0.8 \mu \mathrm{N} \mathrm{m}$, where $0.1 \mu \mathrm{N} \mathrm{m}$ is the limit of the instrument, the corresponding applied stresses are given in Table 6.2). The log-log plots show a linear increase of strain as a function of time for all applied torques.

Each data set in Fig. 6.5 was fit with a power series $y=\mathrm{A} x^{\mathrm{b}}$ (Table 6.2). All fits have a similar power law constant, $b$, of $0.85 \pm 0.03$. Fitting the data yields the applied shear rate (the slope of the curve), for each of the creep experiments, these are given in Table 6.2. 
Using these estimated applied shear rates one can construct a stress vs. shear rate plot (Fig. 6.6).

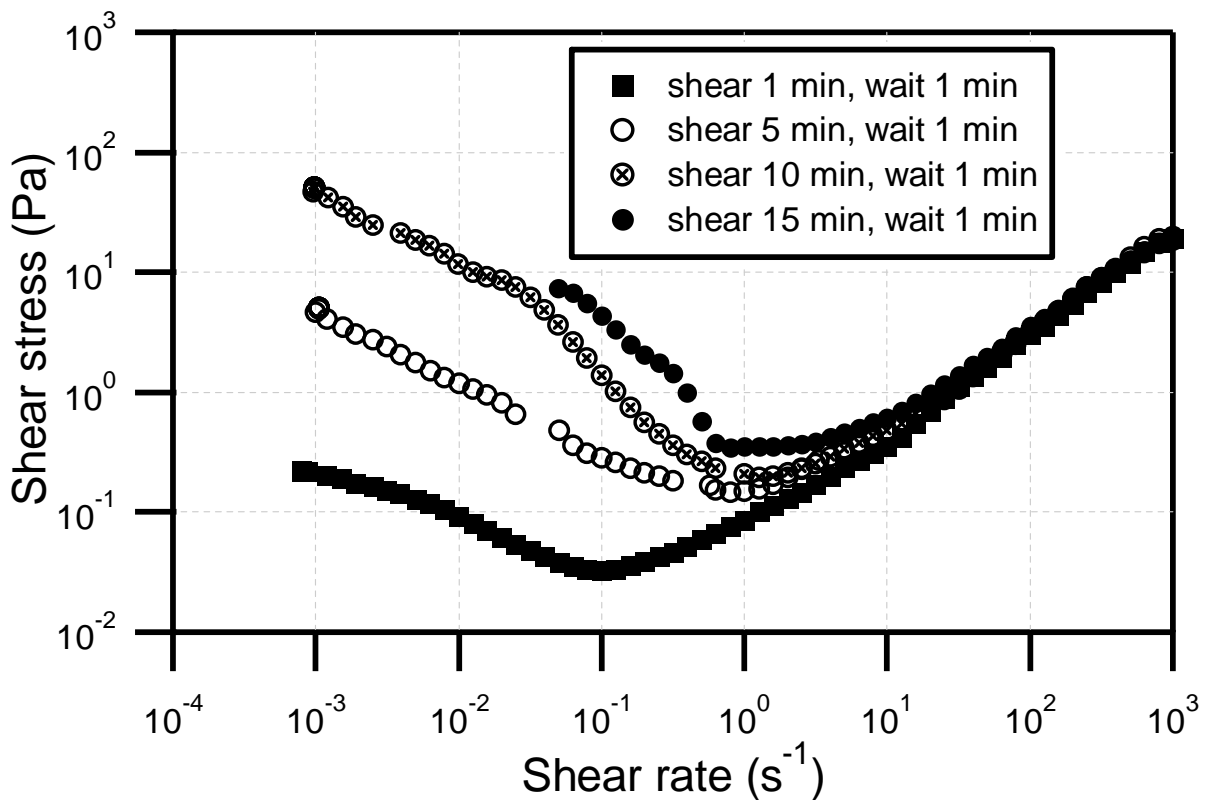

Figure 6.4: Stress vs. shear rate curve for $50 \mathrm{wt} \%$ soybean oil $/ 2 \mathrm{wt} \%$ Na-caseinate $/ 48 \mathrm{wt} \%$ water emulsion during down-stress ramping: 1 min rest for each shear rate, from 1000 to $0.0001 \mathrm{~s}^{-1}$.

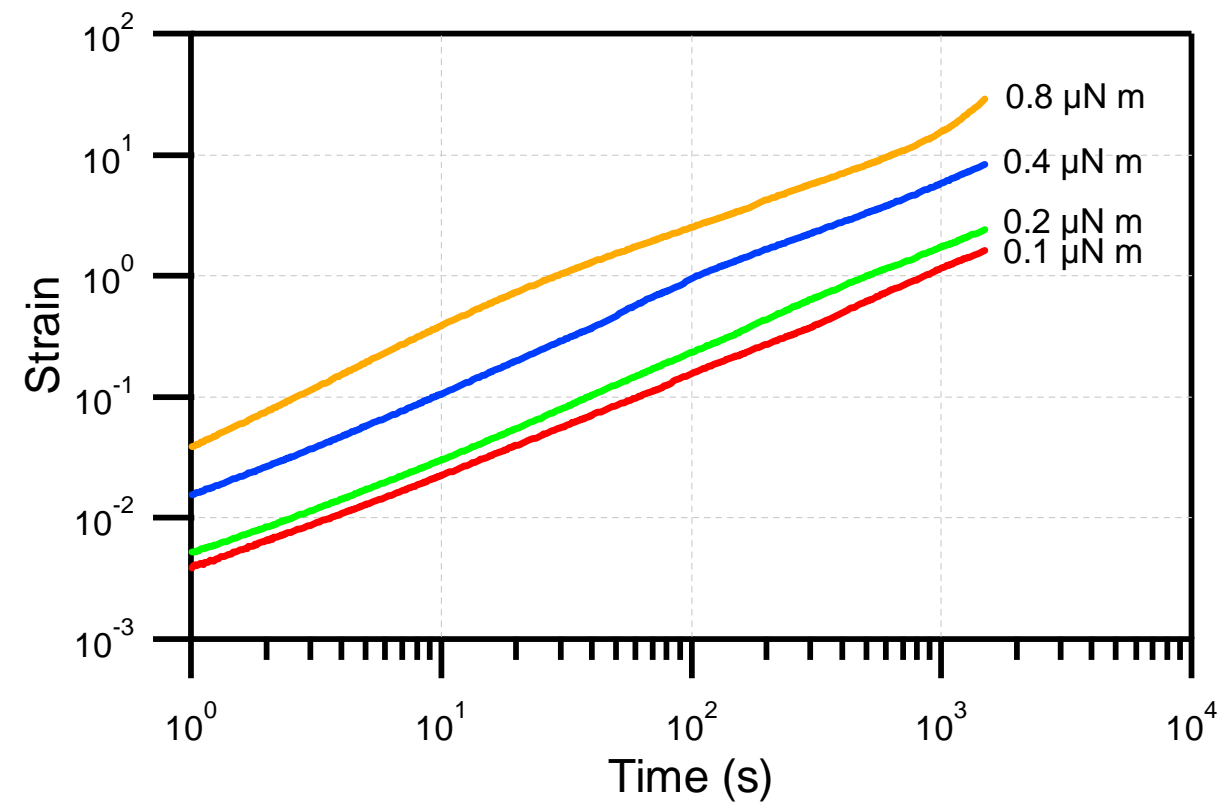

Figure 6.5: Experimental strain response for various applied stresses (i.e., torques) as a function of time for 50 $\mathrm{wt} \%$ soybean oil $/ 2 \mathrm{wt} \% \mathrm{Na}$-caseinate $/ 48 \mathrm{wt} \%$ water emulsions. 
Table 6.2: Parameters obtained from creep experiments on region II of a $50 \mathrm{wt} \%$ soybean oil/2 $\mathrm{wt} \%$ Na-caseinate $/ 48 \mathrm{wt} \%$ water emulsions

\begin{tabular}{cccc}
\hline Torque $(\mu \mathrm{N} \mathrm{m})$ & $\sigma(\mathrm{Pa})^{\mathrm{a}}$ & $\mathrm{b}^{\mathrm{b}}$ & $\dot{\gamma}\left(\mathrm{s}^{-1}\right)^{\mathrm{b}}$ \\
\hline 0.1 & 0.0017 & $0.8291 \pm 0.0011$ & 0.00346 \\
0.2 & 0.0035 & $0.8635 \pm 0.0012$ & 0.00440 \\
0.4 & 0.0071 & $0.8785 \pm 0.0014$ & 0.01457 \\
0.8 & 0.0140 & $0.8482 \pm 0.0028$ & 0.04848
\end{tabular}

${ }^{\mathrm{a}}$ : obtained from direct conversion of the applied torque

${ }^{\mathrm{b}}$ : obtained from power law fits of the data shown in Fig. 6.5

Fitting this shear stress vs. shear rate data with a power series allows empirical classification of the system as shear-thinning, Newtonian or shear-thickening for the power law exponent $<1,=1$ or $>1$, respectively. Fig. 6.6 shows the linear fit to the log-log plot of the experimental stress (applied torque) at each of the four estimated shear rates. With the corresponding power law exponent being equal to 0.71 , the response of the material in region II can be interpreted as shear-thinning. Because the minimum torque that the instrument can apply in a creep experiment is $0.1 \mu \mathrm{N} \mathrm{m}$ (shear rate $=0.00346 \mathrm{~s}^{-1}$ ), which is greater than that required to probe region I we were unable to conclusively establish the existence of a yield stress.

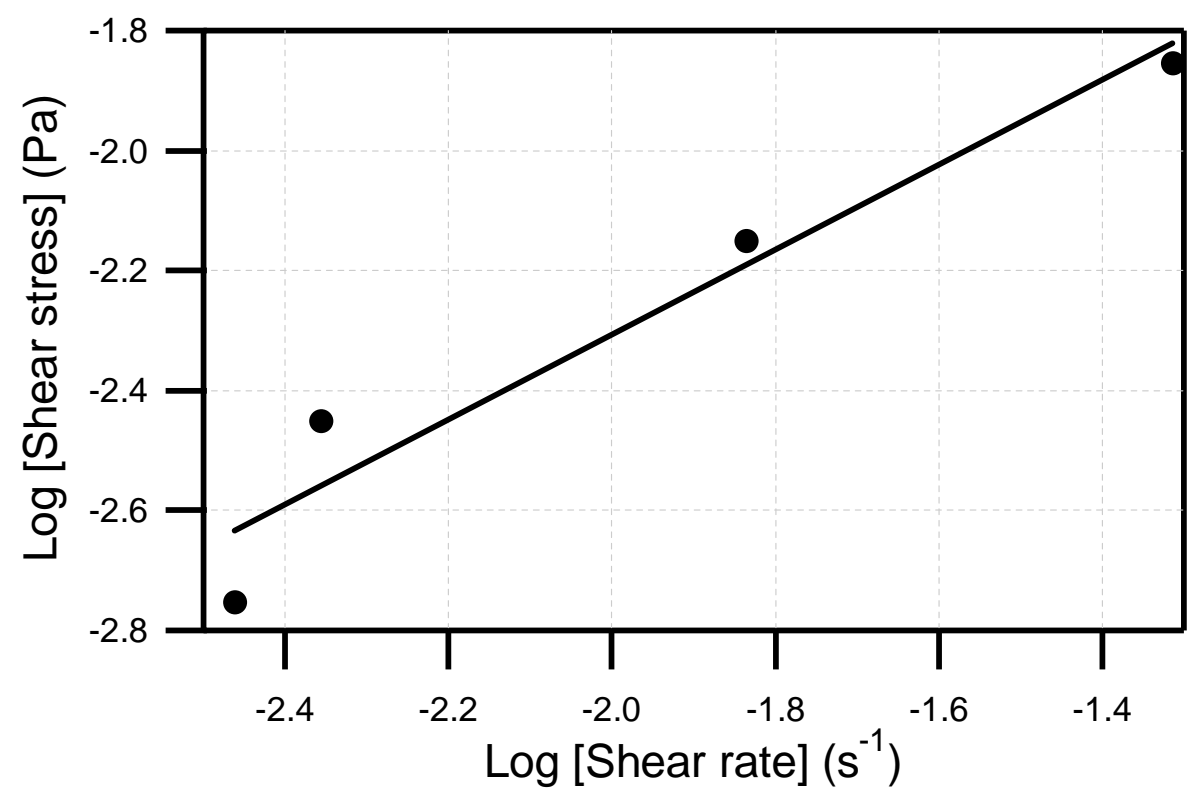

Figure 6.6: Shear stress as a function of estimated shear rate (see Fig. 6.5 and Table 6.2). Solid line corresponds to the linear fit of the $\log \sigma \mathrm{vs} . \log \dot{\gamma}$ plot. The slope of the fit $=0.71$ and indicates that over this range of applied torques the system displays shear-thinning behaviour. 
The transition of the shear-thinning region moving into the stress plateau was investigated by subjecting the sample to a constant shear rate, ranging from 0.01 to $0.1 \mathrm{~s}^{-1}$ (note that the lower shear rate bound of the stress plateau, as estimated directly from the flow curve, for this sample is $\dot{\gamma}_{1}=0.02 \mathrm{~s}^{-1}$ ), for times up to $6000 \mathrm{~s}$. The stress response of the material follows three distinctly different behaviours as a function of time, as shown in Fig. 6.7. At very short times (i.e., start up), the stress rises as a response to the onset of shearing and is more pronounced at shear rates within the stress plateau. The maximum stress achieved is shear rate dependent in this start up period. Subsequent to this start up, the stress decreases and settles down to a constant value. These three behaviours are exhibited at all investigated shear rates. For shear rates corresponding to the shear-thinning region $\left(0.01 \mathrm{~s}^{-1}\right.$ in Fig. 6.7) the equilibrium value is obtained within a few hundred seconds. In contrast, at $0.025 \mathrm{~s}^{-1}$, which is close to the transition boundary between the shear-thinning region and the stress plateau, a slow decay to the equilibrium stress value is seen, which is finally reached at $\sim 4300$ s. When well within the stress plateau (e.g., a shear rate of $0.063 \mathrm{~s}^{-1}$ ), the stress overshoots dramatically at short times and the movement towards equilibrium displays time dependent features, with equilibrium being attained by $\sim 5000 \mathrm{~s}$. We propose that this longtime constant to achieve equilibrium is characteristic of the stress plateau and is a feature that allows determination of the stress plateau onset.

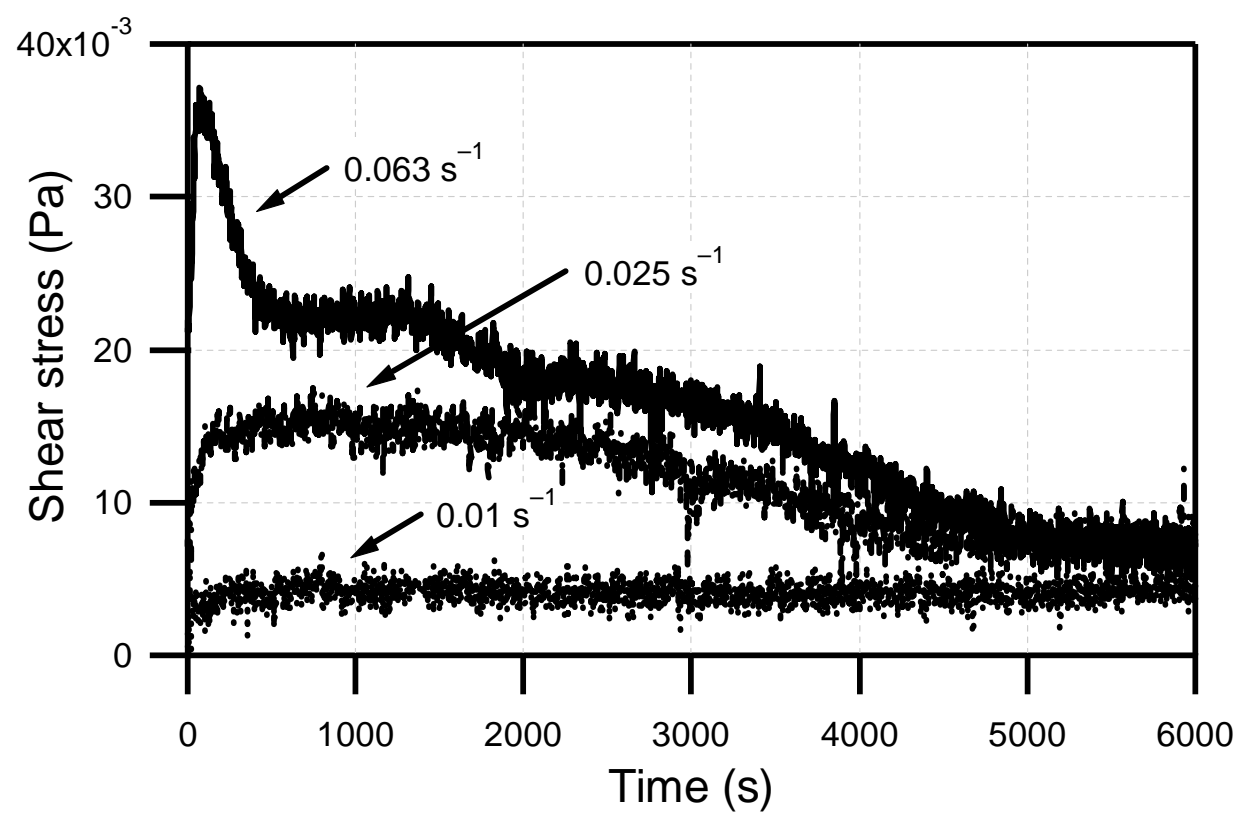

Figure 6.7: Experimental stress response of the system within regions II and III (Fig. 6.1) of the flow curve to an applied shear rate as a function of time for $50 \mathrm{wt} \%$ soybean oil $/ 2 \mathrm{wt} \% \mathrm{Na}$-caseinate $/ 48 \mathrm{wt} \%$ water emulsions. 
Further empirical evidence for the existence of shear-thinning followed by a stress plateau and finally Newtonian behaviour is seen in the flow curve data, obtained under stress peak hold conditions (Fig. 6.8). Each of the three regions of the flow curve: shear-thinning, stress plateau, and Newtonian produce characteristic stress responses as a function of shearing time. As for the data reported above however, analysis of these data to determine rigorously the transition shear rates is difficult due to small fluctuations in the stress response of the sample with time, irrespective of which region of the flow curve is being considered.

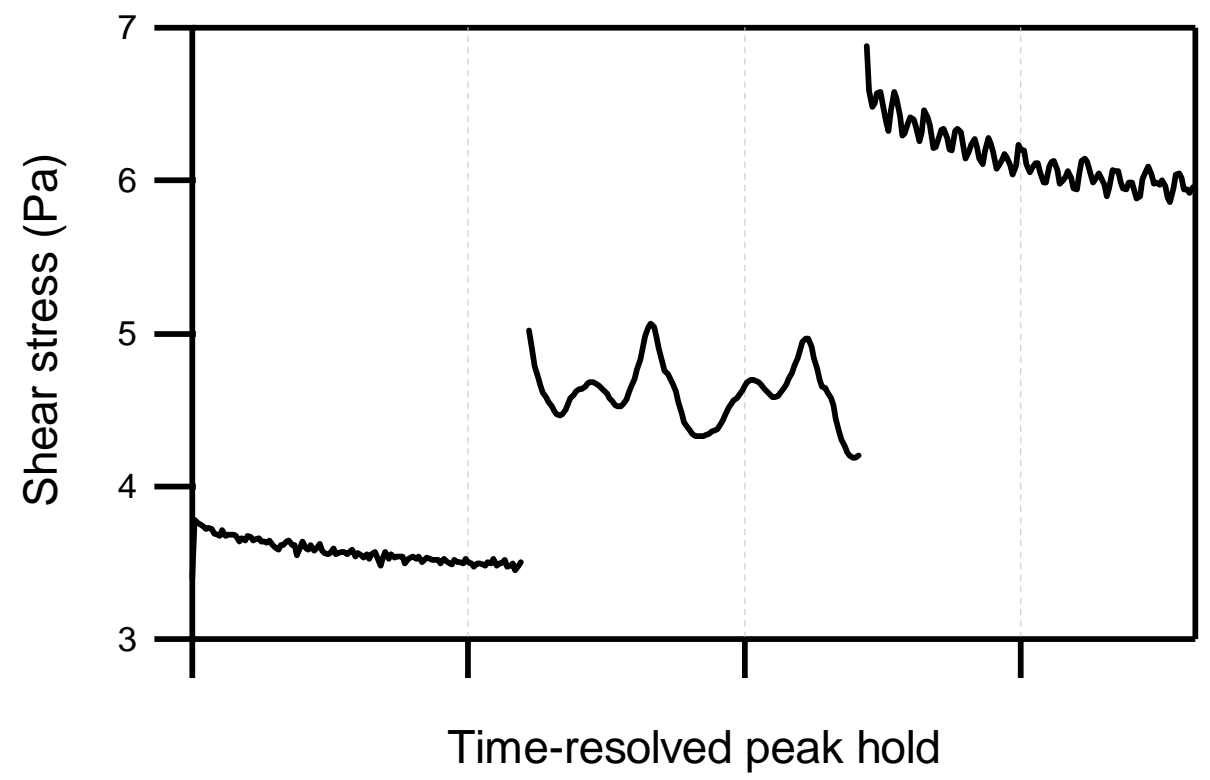

Figure 6.8: Experimental stress response under stress peak hold conditions for $55 \mathrm{wt} \%$ soybean oil/4 wt $\% \mathrm{Na}-$ caseinate $/ 41 \mathrm{wt} \%$ water emulsions. Left to right: shear-thinning, plateau, Newtonian responses.

\subsubsection{NMR Velocimetry}

Due to the low viscosity of emulsions prepared with lower soybean oil and Na-caseinate concentrations, only those emulsions containing high soybean oil and Na-caseinate content were investigated using NMR velocimetry. The $55 \mathrm{wt} \%$ soybean oil $/ 3 \mathrm{wt} \% \mathrm{Na}$-caseinate $/ 42$ $\mathrm{wt} \%$ water emulsion system will be used to demonstrate our NMR velocimetry findings. We have utilised a time-averaged one-dimensional H-1 NMR velocity imaging method to visualise steady state shear-banded velocity profiles.

Each velocity profile displayed in Fig. 6.9 represents a two minutes average velocity profile for a finite volume of the sample, at the specified shear rate. At low shear rates (Fig. 6.9 (a) to (c)), the material is divided into two discontinuous shear bands. A high shear band 
is present near the inner rotor, and a low shear band exists toward the outer stator. The occurrence of this shear-banded state is consistent with the existence of flow inhomogeneity hinted by the stress plateau in the flow curve (Fig. 6.2). At applied shear rates of $2.14 \mathrm{~s}^{-1}$ (Fig. 6.9 (d)) the shear flow is homogeneous across the gap with a Newtonian flow profile; this corresponds to region IV in Fig. 6.1. The inner wall velocity corresponds well with the applied shear rate, an indication that there is little slip.

(a)

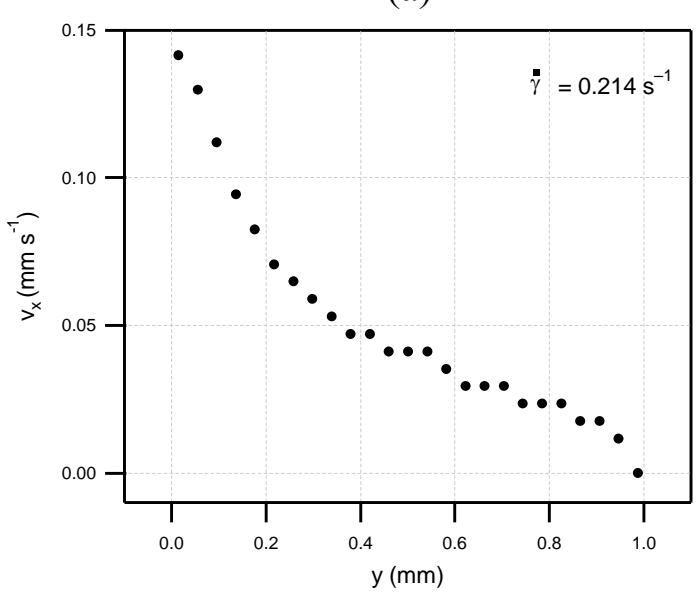

(c)

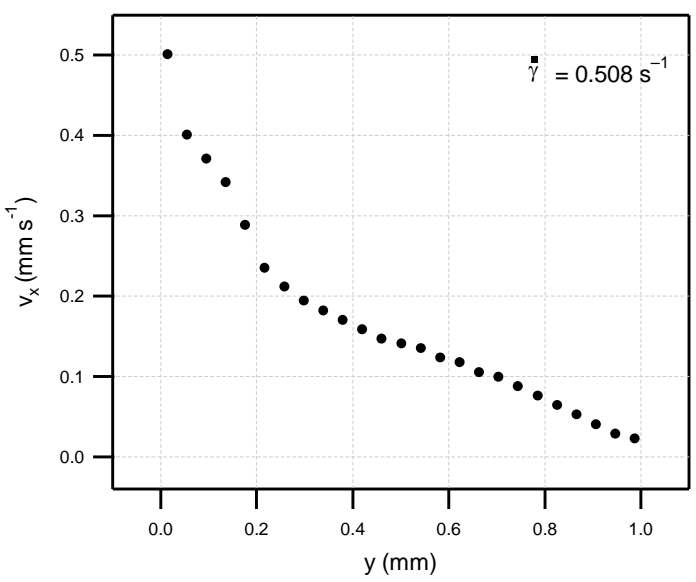

(b)

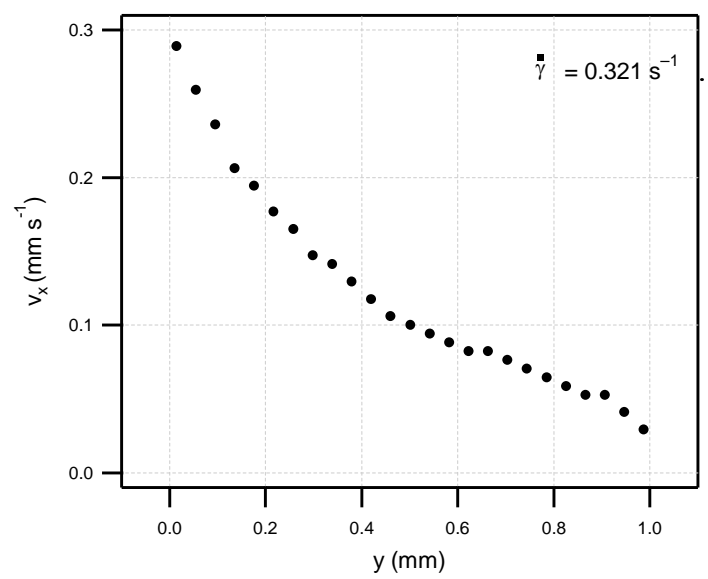

(d)

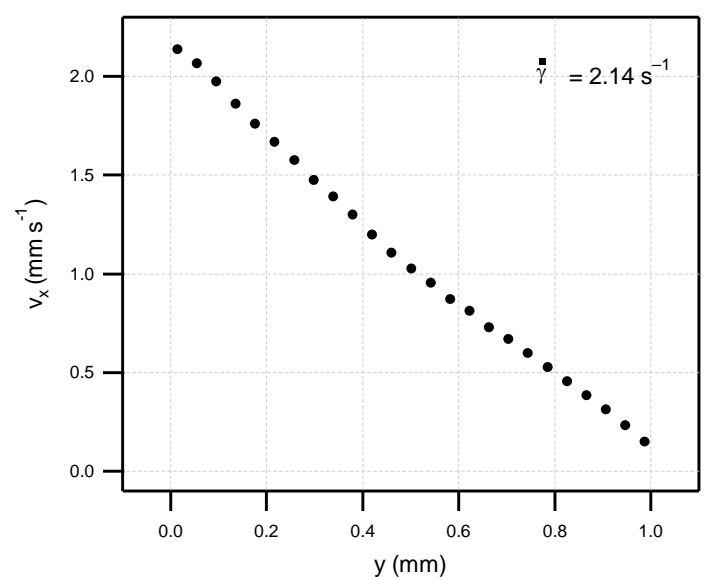

Figure 6.9: Time-averaged one-dimensional velocity profiles for $55 \mathrm{wt} \%$ soybean oil $/ 3 \mathrm{wt} \% \mathrm{Na}$-caseinate $/ 42$ $\mathrm{wt} \%$ water emulsion over a range of applied shear rates. $\mathrm{y}$ is the radial distance from the inner rotor to the outer stator.

The variation in the calculated local shear rates for the two bands as a function of applied shear rate within the stress plateau (Fig. 6.2) is shown in Fig. 6.10. The shear rates were obtained directly from the NMR velocity profiles. We note that the increase in local shear rate as a function of applied shear rate is a linear relationship. The corresponding 
ensemble gap-average shear rate is weighted by the volume fractions of the two bands.

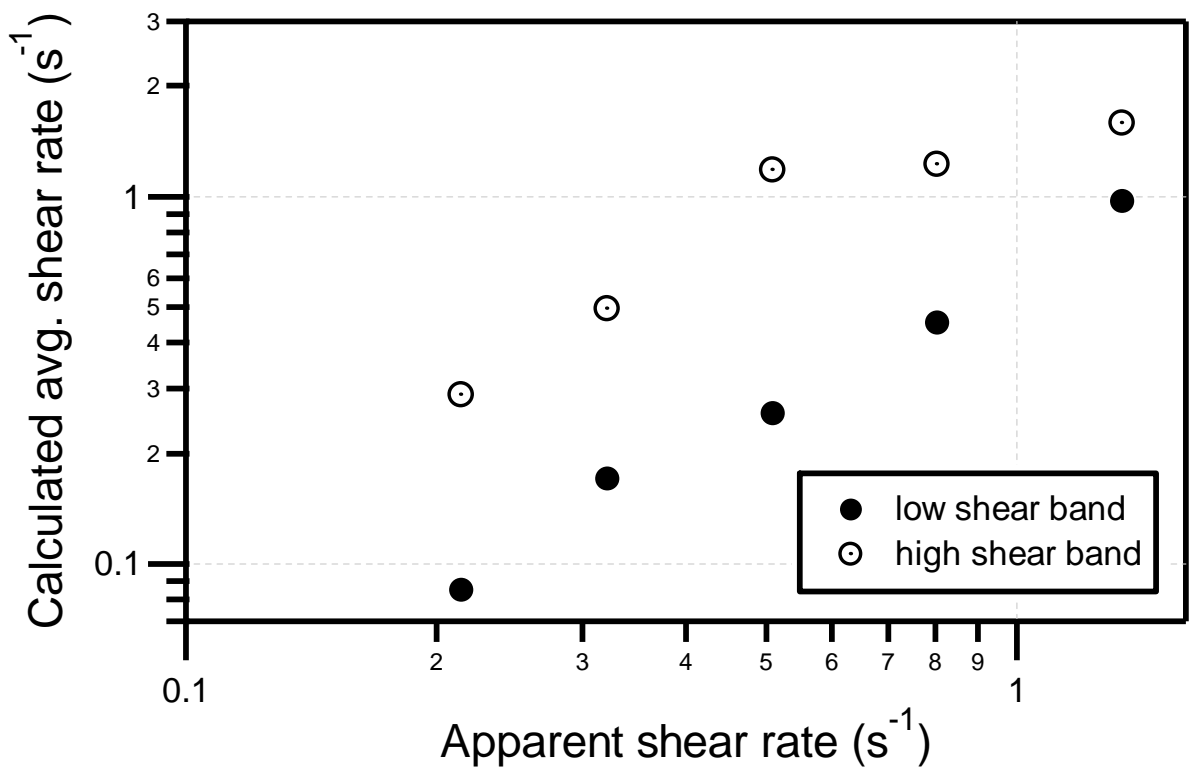

Figure 6.10: Calculated average shear rate for high shear band (near inner rotor) and low shear band (near outer stator) over a range of applied shear rates.

Fig. 6.11 shows the estimated radial position corresponding to the interface between the two shear bands, $\mathrm{y}_{\mathrm{c}}$ as a function of the applied shear rate. The position of the interface remains approximately constant for applied shear rates within the stress plateau (Fig. 6.2).

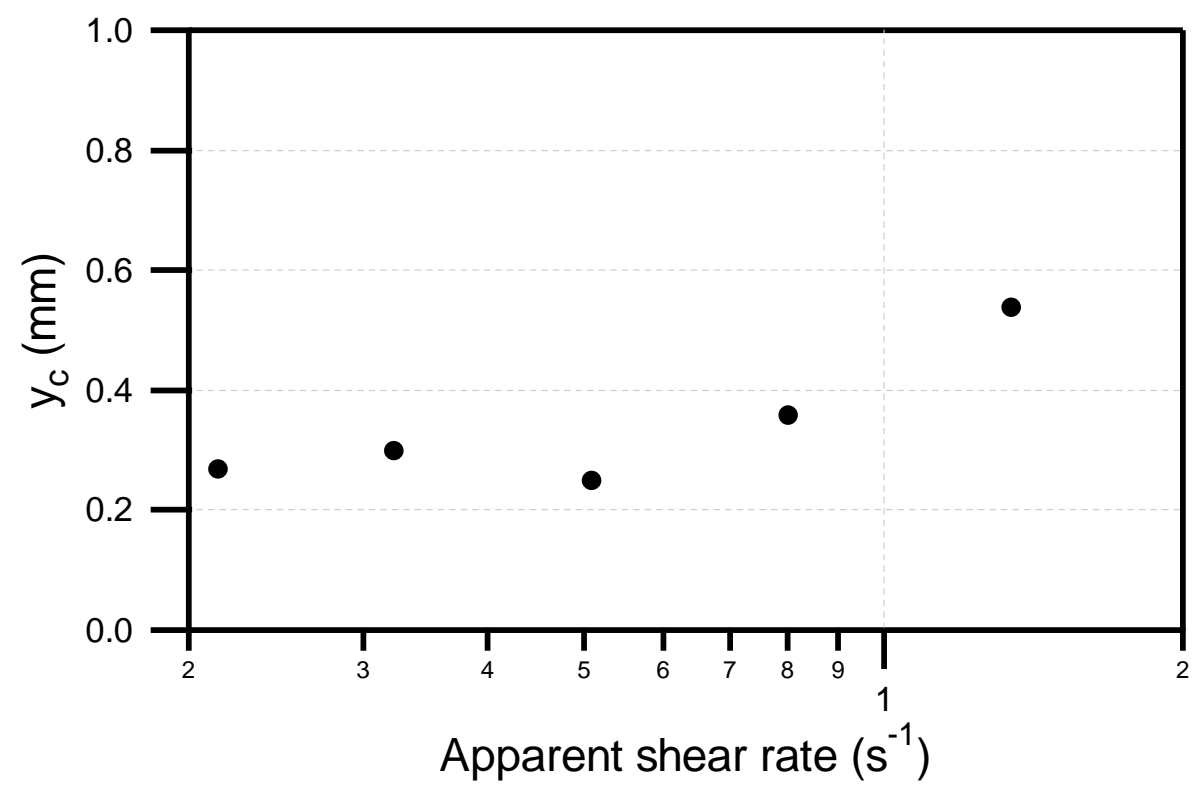

Figure 6.11: Estimated values of $y_{c}$, the interface between the two shear bands, as a function of applied shear rates. 


\subsection{Discussion}

Despite different emulsion domains being evident within the phase space spanned a single steady state stress vs. shear rate response is observed for all emulsions, as exemplified by the data shown in Fig. 6.1, which can be divided into four regions. On increasing shear rate the evolving responses are an apparent yield stress (region I), a shear-thinning region (region II), a stress plateau (region III) and Newtonian flow (region IV). Since these responses occur irrespective of emulsion composition and microstructure, the structure of the interfacial thin film and/or the three-dimensional packing of the emulsion droplets are not fundamental drives for the observed form of the flow curve.

Region I of the shear stress response appears to correspond to yield stress fluid behaviour which is associated with small, irreversible rearrangements, indicative of an elastoplastic property of the sample ${ }^{36,37}$. Slippage in emulsions due to the interactions between the droplets and repulsive interactions of the droplets with the wall leading to the presence of a thin depletion layer adjacent to the wall has been reported previously ${ }^{38-51}$. However, because the corresponding stress in region I is so low the extent of the influence of slippage to the rheological behaviour in region I is not able to be determined. The observed shear-thinning (pseudoplastic) behaviour in region II, on further increasing applied shear rates, is due to the break up of domains of aggregated structure, i.e., a phenomenon occurring on a length scale many times longer than that of the individual droplets. The temporarily trapped continuous phase of the emulsion is subsequently released leading to a reduction in the apparent stress/viscosity ${ }^{30}$. The floc structure is then completely destroyed at much higher applied shear rates, which is indicated by a Newtonian region (region IV) where the apparent viscosity is constant.

For all emulsion samples the stress plateau occurs over approximately two decades of shear rate $\left(\sim 10^{-2}\right.$ to $\left.1 \mathrm{~s}^{-1}\right)$. As such, the critical shear rates defining the plateau are not dependent on oil volume fraction or interdroplet interactions. Probing the dependence of the plateau on droplet size is not possible within the soybean oil system as the distribution is invariant.

The palm oil and tetradecane data confirm that interdroplet interactions, emulsion microstructure and the structure of the interfacial thin film are not the primary driving forces behind the observed stress plateau, since all of these are altered on switching oil. The onset 
of the stress plateau is therefore set by a specific switch in the flow characteristics of the system as suggested by Ovarlez et al. ${ }^{34}$. The dominance of two or more effects is changed under particular flow conditions. For the systems investigated here this corresponds to a particular shear rate and applied shearing time.

The ageing of a system under flow and therefore the dependence of the flow curve on the time of the applied shear rate, coupled with the existence of a stress plateau, as seen for the concentrated emulsions investigated here, has recently been described for another soft glassy material, namely star polymers above their critical concentration ${ }^{35}$. The flow curves of the star polymers for short shearing times display a near Newtonian response for all investigated shear rates. On increasing the shearing time up to $30000 \mathrm{~s}$ (as compared with our $900 \mathrm{~s}$ ) the stress response evolved yielding a stress plateau at low shear rates and then the expected Newtonian response. Competition between ageing and rejuvenation, which could be readily altered in this thermodynamically stable system, as compared with kinetically controlled emulsions investigated here, was shown in the star polymer system to control the observed flow curve evolution. Because concentrated emulsions undergo macroscopic destabilisation over time, full manipulation of the system to explore this as the origin of the time evolution of the stress response could not be fully investigated. However given the close similarity of the observed evolution in the two soft glassy systems it is likely that this is also the origin here.

While the time constant for the formation of the stress plateau is not influenced by the local features of the emulsions, the stress response of the materials is a function of Nacaseinate concentration (Fig. 6.3), though not oil volume fraction. The dependence is a monotonically increasing function. The underlying microstructure changes are however not evident in the dependence.

High volume fractions are well known to induce thixotropic effects. This means that when the structure formed via droplet packing is disrupted through an applied shear deformation, it is restored some time after the deformation has ceased ${ }^{52}$. However, a thixotropic effect was not observed in our system because a similar stress plateau is absent in the down-ramping sweep (Fig. 6.4). Moving down from the high shear rates branch toward the stress plateau, the shear rate is always maintained above the critical shear rate $\left(\dot{\gamma}_{2}\right)$, thereby acting as a pre-shear, or stress plateau inhibitor. We note that for shearing times as long as 15 minutes a stress plateau is almost evident. Unfortunately due to the kinetic nature of the emulsion systems studied herein, the extent of shearing in a down-ramping sweep for 
such long shearing times begins to degrade the sample. Thus, reliable data cannot be obtained below $\sim 5 \times 10^{-2} \mathrm{~s}^{-1}$. Furthermore, for longer shearing times, reliable data outside the Newtonian region cannot be obtained. On a down-ramping sweep, if the shearing time is insufficient at a particular shear rate to obtain a steady state response before proceeding to a lower shear rate, a dip in the flow curve is observed (i.e., an undershoot occurs), since the stress tends to rise toward the stress plateau value. We note that this undershoot is modified by ageing effects. It would be interesting to carry out stress relaxation experiment before and at the plateau region to explore the correlation between the shear response and the microstructure of the samples.

Fig. 6.7 reflects the stress evolution of fresh samples at different shear rates within region II and III, as defined in Fig. 6.1. Although direct comparison between this data and the response of the material as shown in Fig. 6.1 is not strictly applicable (since Fig. 6.1 exhibits a complex nature of the interplay between shear effects and ageing), we found that there is a long-time constant (thousands of seconds) for the resulting stress before settling down to an equilibrium value for shear rates within the stress plateau. In contrast, within the shearthinning region equilibrium is achieved within a few hundred seconds. It is difficult to interpret the competition between the restructuring and destruction rates over such long times and constant shearing. Local and collective rearrangements may lead to continual break up and renewal of long length scale aggregates of oil domains, which will thereby be responsible for the observed stress fluctuations as a function of time, while the effect of continual flow of the sample will result in deformation of the oil droplets leading to a decrease in the observed stress $^{37}$.

The stress plateau (region III), which lies between a low and a high shear rates branch of the stress profile, has been suggested as developing from an underlying flow curve with a negative slope $\mathrm{e}^{31}$. Such a stress plateau is often indicative of a shear banding phenomenon. The data may be fit using a number of different phenomenological models (e.g., the Cross model $^{53}$ and DJS model ${ }^{54,55}$ ) however while good fits to the data may be obtained, nonphysical constants are returned, or if physically appropriate values are applied the critical shear rates are poorly defined. One of the plausible explanations for the formation of a stress plateau is the existence of unstable flow $^{32}$, which might intrinsically arise from the interparticle interactions. The transition between the stress plateau and Newtonian regions indicates a significant change in the flow behaviour, with evidence obtained by the velocity profiles shown in Fig. 6.9. In Fig. 6.9, we observed a transition from fluids subdivided into 
two distinct bands to an extension of uniform fluidised behaviour throughout the gap. This transition in the velocity profile occurs at the applied shear rates in the region where the flow curve exhibits an upturn in stress (see Fig. 6.2). Rejuvenation is likely to happen in this high shear rates branch ${ }^{56}$. The discontinuous velocity profiles therefore confirm that the stress plateau is due to the presence of a shear-banded state.

With the existence of shear banding established our data support the proposal of Ovarlez et al. that shear banding is a universal phenomenon that should be observed in complex structured materials and/or those exhibiting non-linear rheological behaviour ${ }^{34}$. And that furthermore the origin of the shear-banded state is due to the switch in dominance between at least two competing effects occurring under specific flow conditions.

In order to understand the origin of the shear banding in our complex material we consider the model proposed by Coussot and co-workers (see 22 for detailed description). This model is based on competition between restructuring and destruction rates, used to generally describe the response of the materials studied over the whole range of applied shear rates. The shear-banded state is manifested due to the switching dominance of the two rates occurring at a particular shear rate correlating to the onset of the banded state. In a simplistic way, one can attribute the viscosity of a material to its instantaneous structure. When subjected to flow, this will result in the difference between the restructuring rate (mainly material dependent) and the destruction rate (mainly depending on the instantaneous shear rates). At low applied shear rates, the restructuring rate dominates. Here the emulsion optimises its flow response by rearranging the large length scale oil droplet aggregates, thereby changing the emulsion macrostructure but its microstructure remains unchanged. The rate of destruction dominates at higher shear rates. This results in the acceleration of the flow and the corresponding viscosity. Macroscopic domains begin to be broken up, this finally leading to a Newtonian response where complete macroscopic destruction of the material has occurred.

\subsection{Conclusions}

A shear-banded state is achieved in polydisperse emulsions within the concentrated domain of the soybean oil, palm oil and tetradecane/Na-caseinate/water systems. This banded state arises independently of macroscopic phase behaviour. The stress plateau is bounded by a 
shear-thinning region at lower shear rates, and Newtonian behaviour at higher shear rates. Shearing time is an important parameter in achieving a truly steady state response with the data presented here indicating that emulsions may in general display more complex flow behaviour than has hither too been indicated, more closely resembling those of soft glassy materials. The shear banding is the result of competition between restructuring and destruction of macroscopic domains of oil droplet aggregates. The onset of the shear-banded state is independent of oil volume fraction, droplet size, interdroplet interactions and emulsion microstructure. Our evidence for the occurrence of shear banding in concentrated emulsions lends credence to the possible universality of this phenomenon in complex soft glassy materials. 


\subsection{References}

1. J. W. Goodwin and W. B. Russel, Curr. Opin. Colloid Interface Sci. 2, 409-410 (1997).

2. T. D. Dimitrova and F. Leal-Calderon, Adv. Colloid Interface Sci. 108-109, 49-61 (2004).

3. D. Gabriele, M. Migliori, R. Di Sanzo, C. O. Rossi, S. A. Ruffolo and B. de Cindio, Food Hydrocolloids 23, 619-628 (2008).

4. E. Dickinson and M. Golding, J. Colloid Interface Sci. 197, 133-141 (1998).

5. C. G. Quintero, C. Noik, C. Dalmazzone and J.-L. Grossiord, Rheol. Acta 47, 417-424 (2008).

6. A. R. Taherian, P. Fustier and H. S. Ramaswamy, Int. J. Food Prop. 11, 24-43 (2008).

7. G. B. Thurston and S. S. Davis, J. Colloid Interface Sci. 69, 199-208 (1979).

8. E. Dickinson and J. Chen, J. Agric. Food Chem. 46, 91-97 (1998).

9. S. R. Derkach, S. M. Levachev, A. N. Kukushkina, N. V. Novoselova, A. E. Kharlov and V. N. Matveenko, Colloid J. 68, 700-706 (2006).

10. S. M. Levachev, A. E. Harlov, A. N. Kukuchkina, N. V. Novoselova, V. N. Matveenko and S. R. Derkach, Vestn. Mosk. Univ., Ser. 2: Khim. 47, 218-222 (2006).

11. A. Y. Malkin, I. Masalova, P. Slatter and K. Wilson, Rheol. Acta 43, 584-591 (2004).

12. I. Masalova and A. Y. Malkin, Colloid J. 69, 185-197 (2007).

13. M. F. San Martin-Gonzalez, A. Roach and F. Harte, Food Sci. Tech. 42, 301-311 (2009).

14. J. Mewis and A. J. B. Spaull, Adv. Colloid Interface Sci. 6, 173-200 (1976).

15. R. B. Bird, D. Gance and B. J. Yarusso, Rev. Chem. Eng. 1, 1-70 (1983).

16. B. P. Binks, J. H. Clint and C. P. Whitby, Langmuir 21, 5307-5316 (2005).

17. F. Pignon, A. Magnin and J.-M. Piau, J. Rheol. 40, 573-587 (1996).

18. A. J. Liu and S. R. Nagel, Nature 396, 21-22 (1998).

19. D. M. Mueth, G. F. Debregeas, D. F. Karczmar, P. J. Eng, S. R. Nagel and H. M. Jaeger, Nature 406, 385-389 (2000).

20. W. Losert, L. Bocquet, T. C. Lubensky and J. P. Gollub, Phys. Rev. Lett. 85, 14281431 (2000). 
21. G. Debrégeas, H. Tabuteau and J.-M. di Meglio, Phys. Rev. Lett. 87, 178305-178308 (2001).

22. P. Coussot, J. S. Raynaud, F. Bertrand, P. Moucheront, J. P. Guilbaud and H. T. Huynh, Phys. Rev. Lett. 88, 218301-218304 (2002).

23. S. A. Rogers, D. Vlassopoulos and P. T. Callaghan, Phys. Rev. Lett. 100, 128304128307 (2008).

24. T. Gibaud, C. Barentin and S. Manneville, Phys. Rev. Lett. 101, 258302-258305 (2008).

25. L. Bécu, S. Manneville and A. Colin, Phys. Rev. Lett. 96, 138302-138305 (2006).

26. L. Isa, R. Besseling and W. C. K. Poon, Phys. Rev. Lett. 98, 198305-198308 (2007).

27. J. Goyon, A. Colin, G. Ovarlez, A. Ajdari and L. Bocquet, Nature 454, 84-87 (2008).

28. J. P. Decruppe, R. Cressely, R. Makhloufi and E. Cappelaere, Colloid Polym. Sci. 273, 346-351 (1995).

29. R. W. Mair and P. T. Callaghan, Europhys. Lett. 36, 719-724 (1996).

30. S. M. Fielding, Phys. Rev. E 76, 016311-016318 (2007).

31. N. A. Spenley, M. E. Cates and T. C. B. McLeish, Phys. Rev. Lett. 71, 939-942 (1993).

32. J. Yerushalmi, S. Katz and R. Shinnar, Chem. Eng. Sci. 25, 1891-1902 (1970).

33. P. C. F. Møller, S. Rodts, M. A. J. Michels and D. Bonn, Phys. Rev. E 77, 041507$041511(2008)$.

34. G. Ovarlez, S. Rodts, X. Chateau and P. Coussot, Rheol. Acta 48, 831-844 (2009).

35. S. A. Rogers, P. T. Callaghan, G. Petekidis and D. Vlassopoulos, J. Rheol. 54, 133$158(2010)$.

36. J. N. Roux and G. Combe, C.R. Physique 3, 131-140 (2002).

37. F. D. Cruz, F. Chevoir, D. Bonn and P. Coussot, Phys. Rev. E 66, 051305-051307 (2002).

38. R. Buscall, J. I. McGowan and A. J. Motron-Jones, J. Rheol. 37, 621-641 (1993).

39. R. Pal, Colloids Surfs. A 162, 55-66 (2000).

40. M. C. Sánchez, C. Valencia, J. M. Franco and C. Gallegos, J. Colloid Interface Sci. 241, 226-232 (2001).

41. J. M. Franco, C. Gallegos and H. A. Barnes, J. Food Eng. 36, 89-102 (1998).

42. A. Yoshimura and R. K. Prud'homme, J. Rheol. 32, 53-67 (1988).

43. C. Gallegos and J. M. Franco, Curr. Opin. Colloid Interface Sci. 4, 288-293 (1999). 
44. S. P. Meeker, R. T. Bonnecaze and M. Cloitre, J. Rheol. 48, 1295-1320 (2004).

45. J.-B. Salmon, L. Bécu, S. Manneville and A. Colin, Eur. Phys. J. E 10, 209-221 (2003).

46. W. B. Russel and M. C. Grant, Colloids Surfs. A 161, 271-282 (2000).

47. U. Yilmazer and D. M. Kalyon, J. Rheol. 33, 1197-1212 (1989).

48. M. M. Robins, A. D. Watson and P. J. Wilde, Curr. Opin. Colloid Interface Sci. 7, 419-425 (2002).

49. A. F. Méndez-Sánchez, M. R. López-González, V. H. Rolón-Garrido, J. PérezGonzález and L. de Vargas, Rheol. Acta 42, 56-63 (2003).

50. P. J. A. Hartman Kok, S. G. Kazarian, B. J. Briscoe and C. J. Lawrence, J. Colloid Interface Sci. 280, 511-517 (2004).

51. H. J. Walls, S. B. Caines, A. M. Sanchez and S. A. Khan, J. Rheol. 47, 847-868 (2003).

52. P. Coussot, H. Tabuteau, X. Chateau, L. Tocquer and G. Ovarlez, J. Rheol. 50, 975994 (2006).

53. M. M. Cross, J. Colloid Sci. 20, 417-437 (1965).

54. M. Johnson and D. Segalman, J. Non-Newtonian Fluid Mechanics 2, 255-270 (1977).

55. P. D. Olmsted, O. Ratulescu and C.-Y. D. Lu, J. Rheol. 44, 257-275 (2000).

56. H. Wassenius and P. T. Callaghan, Eur. Phys. J. E 18, 69-84 (2005). 


\section{Chapter 7}

\section{Palm OilEmulsions}

\subsection{Introduction}

In the previous chapters the microstructural and rheological properties of emulsions in the soybean oil/Na-caseinate/water system were discussed. PGSTE-NMR results indicate that water molecules undergo unrestricted diffusion at all observation times. Each diffusion peak for the dispersed phase corresponds to a different type of motion or combination of motions: unrestricted and restricted diffusion of the oil droplets themselves and restricted diffusion of the oil molecules in the oil droplets. Complex flow behaviour was also evident. A stress plateau arises in the steady state flow curve irrespective of the observed macroscopic phase behaviour. In this chapter we investigate the effects on the emulsion characteristic by changing to another food grade oil which is more temperature sensitive: palm oil.

As a food product, refined and/or fractionated palm oil is usually present as a dispersing medium or as the dispersed phase to achieve certain functional purposes. To date, the majority of palm oil emulsions manufactured are of the water-in-oil type due to its commercial application in margarines and spreads ${ }^{1}$. In comparison, palm oil-in-water emulsions receive very little attention. The studies on properties of palm oil-based emulsions, however, have been reported by a few authors. Among others, are the works of Chow et al. ${ }^{2}$ and Ahmad et al. ${ }^{3}$ where stability, droplet size distribution and interfacial tension of palm oil-in-water emulsion were monitored in the presence of the emulsifier mixtures.

The emulsion phase diagram for the palm oil/Na-caseinate/water system described in Chapter 4 is very similar to that of the soybean oil/Na-caseinate/water system. As such, one might expect palm oil emulsions to be directly comparable with soybean oil emulsions. Despite the strong similarities of the two oils as commercial blends, their exact mix is different and hence there are corresponding changes in the emulsion domains formed on an 
oil/water continuum. The microstructure and responses of palm oil/Na-caseinate/water emulsions with respect to flow will be detailed in the next sections.

\subsection{Results}

The phase diagram for palm oil concentrations $\geq 50 \mathrm{wt} \%$ was reported in Chapter 4 . The following presents only results for the study of emulsions in domains I, II and III.

\subsubsection{Laser Diffraction Particle Sizing}

In Fig. 7.1 is shown the droplet size distributions of freshly prepared $50 \mathrm{wt} \% \mathrm{palm}$ oil/ $\mathrm{Na}-$ caseinate/water emulsions from 1 to $3 \mathrm{wt} \%$ Na-caseinate. The lognormal form of the distributions for all samples is very similar and compare well with the soybean oil system. The bimodal distribution, remains unchanged on increasing Na-caseinate concentration.

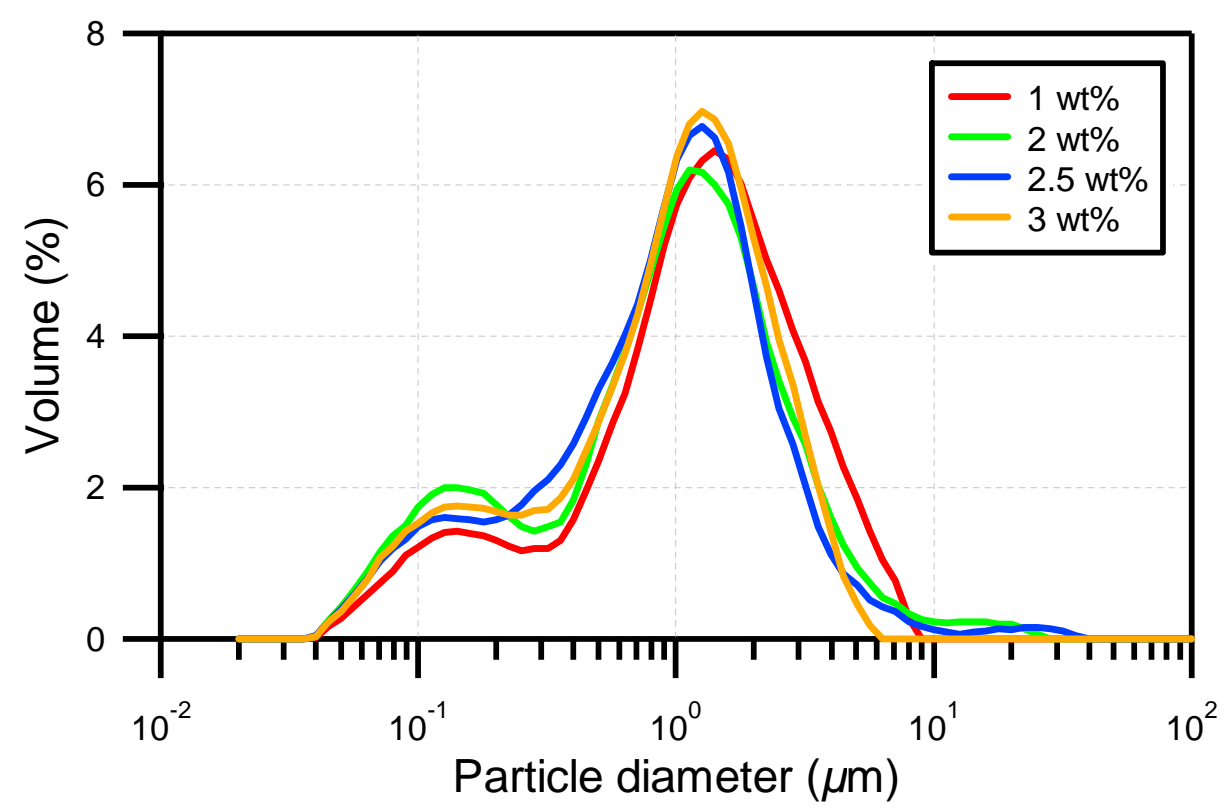

Figure 7.1: Droplet size distributions for freshly prepared $50 \mathrm{wt} \%$ palm oil/Na-caseinate/water emulsions with 1 , 2, 2.5 and $3 \mathrm{wt} \%$ Na-caseinate.

The emulsions are very polydisperse, spanning from $\sim 0.04$ to $9 \mu \mathrm{m}$ in diameter. $D[3,2]$ ranges from 0.39 to $0.50 \mu \mathrm{m}$ while $D[4,3]$ ranges from 1.22 to $1.63 \mu \mathrm{m}$. The two droplet mode diameters are centred around 0.14 and $1.3 \mu \mathrm{m}$, respectively. The third mode is 
occasionally seen at $\sim 18 \mu$ m which may be due to a few large oil droplets or small trapped air bubbles.

In Fig. 7.2 is shown the droplet size distributions of fresh emulsions consisting of 50, 52, 54, 56 and $58 \mathrm{wt} \%$ palm oil/2.5 wt\% Na-caseinate/water. All droplet size distributions for emulsions prepared at a fixed $\mathrm{Na}$-caseinate content but increasing palm oil concentration are very similar. Oil droplets from $\sim 0.04$ to $9 \mu \mathrm{m}$ in diameter exist. The distribution is basically bimodal with peaks at $\sim 0.13$ and $1.3 \mu \mathrm{m}$, respectively. The small right hand side peak at $\sim 18 \mu \mathrm{m}$ is seen consistently for this concentration of Na-caseinate. $D[3,2]$ and $D[4,3]$ values range from 0.41 to $0.59 \mu \mathrm{m}$ and from 1.22 to $1.79 \mu \mathrm{m}$, respectively.

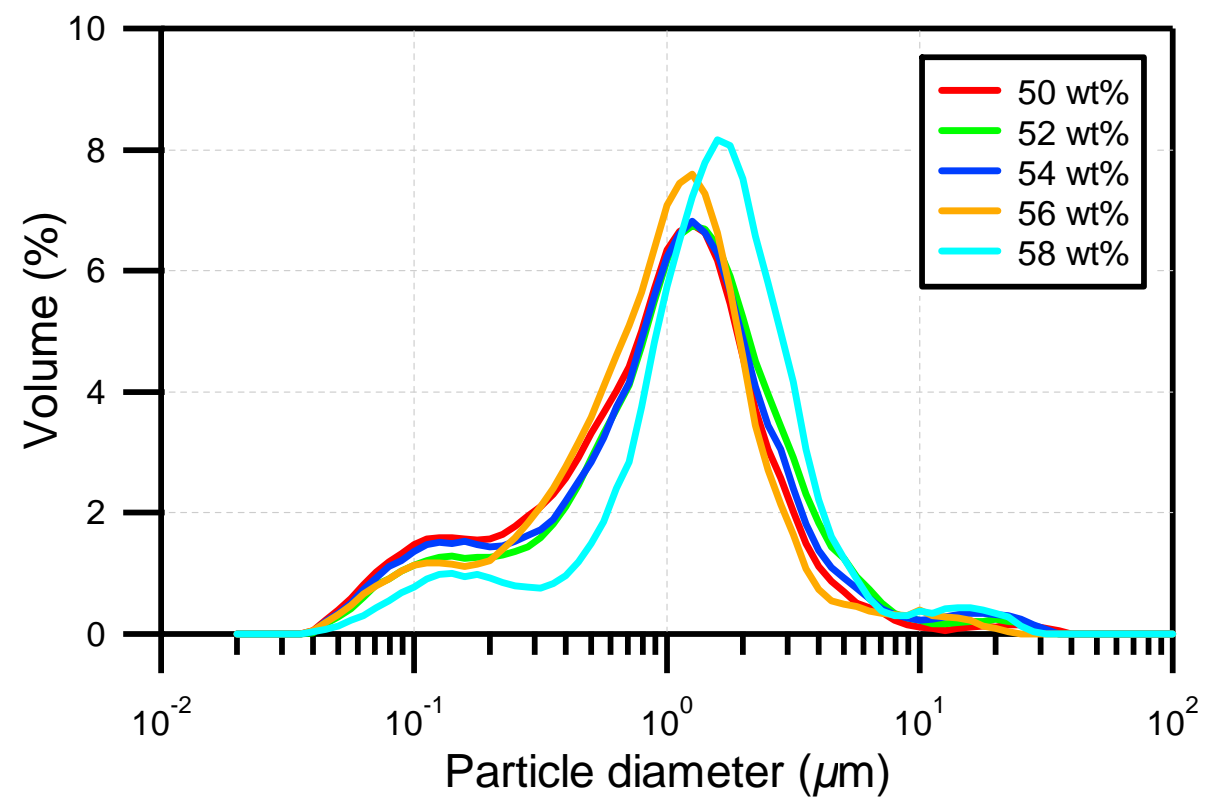

Figure 7.2: Droplet size distributions for freshly prepared palm oil/2.5 wt\% Na-caseinate/water emulsions with $50,52,54,56$ and $58 \mathrm{wt} \%$ palm oil.

\subsubsection{PGSTE-NMR}

For this palm oil system, only the diffusion of water and oil were investigated. The study of Na-caseinate diffusion was not able to be carried out as the proton signals for Na-caseinate are too weak and overlap with those of the proton signals for palm oil.

The attenuation data for water diffusion follow a single exponential decay for all palm oil and Na-caseinate concentrations. In Fig. 7.3 is displayed representative measured diffusion coefficients for water, in the case of $2 \mathrm{wt} \% \mathrm{Na}$-caseinate emulsions, at the observation time, $\Delta$ of $20 \mathrm{~ms}$ upon increasing palm oil concentration. The trend of the 
measured water diffusion coefficient is very similar to that of the soybean oil system. It is a decreasing function of increasing palm oil concentration. Little variation in the diffusion coefficient as compared to Fig. 7.3 has been observed for each concentration of palm oil upon incrementing the values of $\Delta$ up to $200 \mathrm{~ms}$. This reveals that water undergoes unrestricted diffusion for all palm oil concentrations.

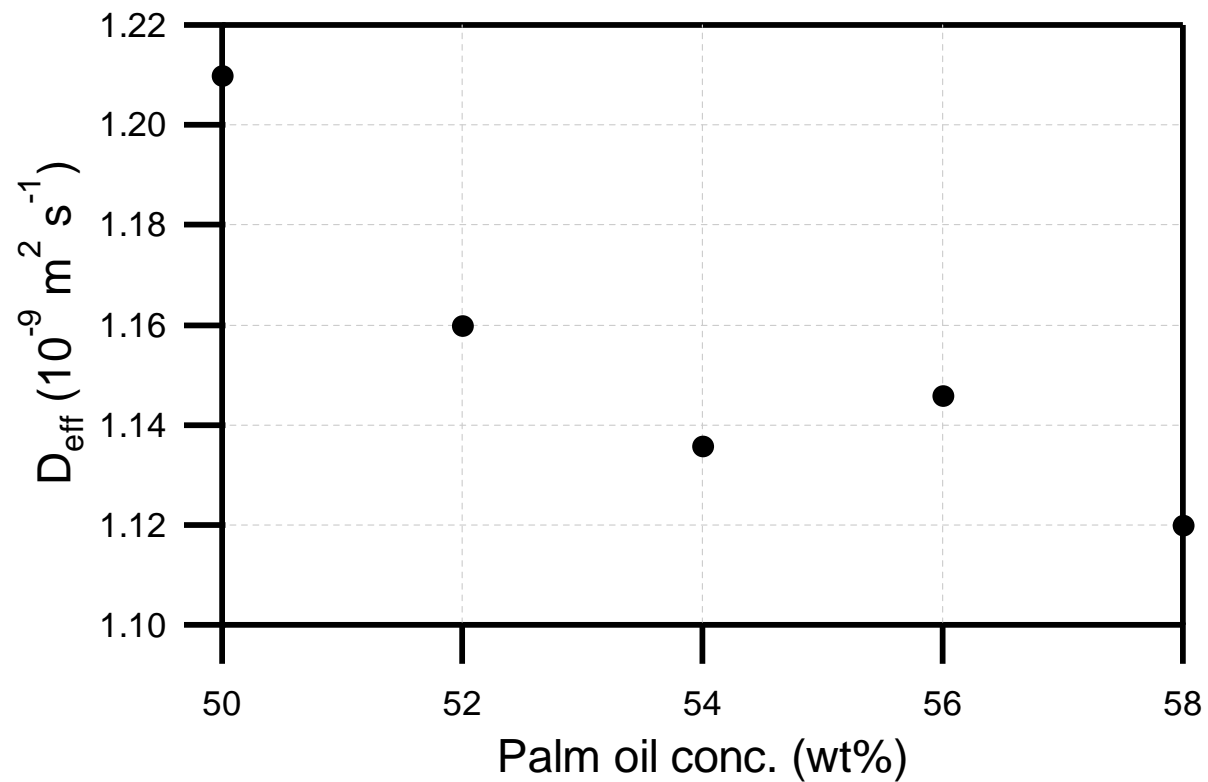

Figure 7.3: Diffusion coefficient of water as a function of increasing palm oil concentration, $\Delta=20 \mathrm{~ms}$, for freshly prepared palm oil $/ 2 \mathrm{wt} \% \mathrm{Na}$-caseinate/water emulsions.

In Fig. 7.4 is shown the diffusion coefficient of water as a function of Na-caseinate concentration, for $50 \mathrm{wt} \%$ palm oil/Na-caseinate/water emulsions at $\Delta=20 \mathrm{~ms}$. The near constant water diffusion coefficient observed for Na-caseinate concentrations $\leq 2.25 \mathrm{wt} \%$ strongly suggest the travelling pathway of water is not altered. This is consistent with the additional Na-caseinate all going to the oil droplet surface and not participating in other structural changes. On increasing Na-caseinate concentrations from $2.25 \mathrm{wt} \%$, the dramatic decrease in measured diffusion coefficient of water correlates well with the macroscopic phase transition, i.e., from domain I to II in the phase diagram of this palm oil system (see Chapter 4) with the additional Na-caseinate now inducing enhanced interactions within the system impeding water diffusion. As the measured diffusion coefficient of water for all samples remains on the same order of magnitude as that of bulk water, this indicates that they are oil-in-water emulsions. 


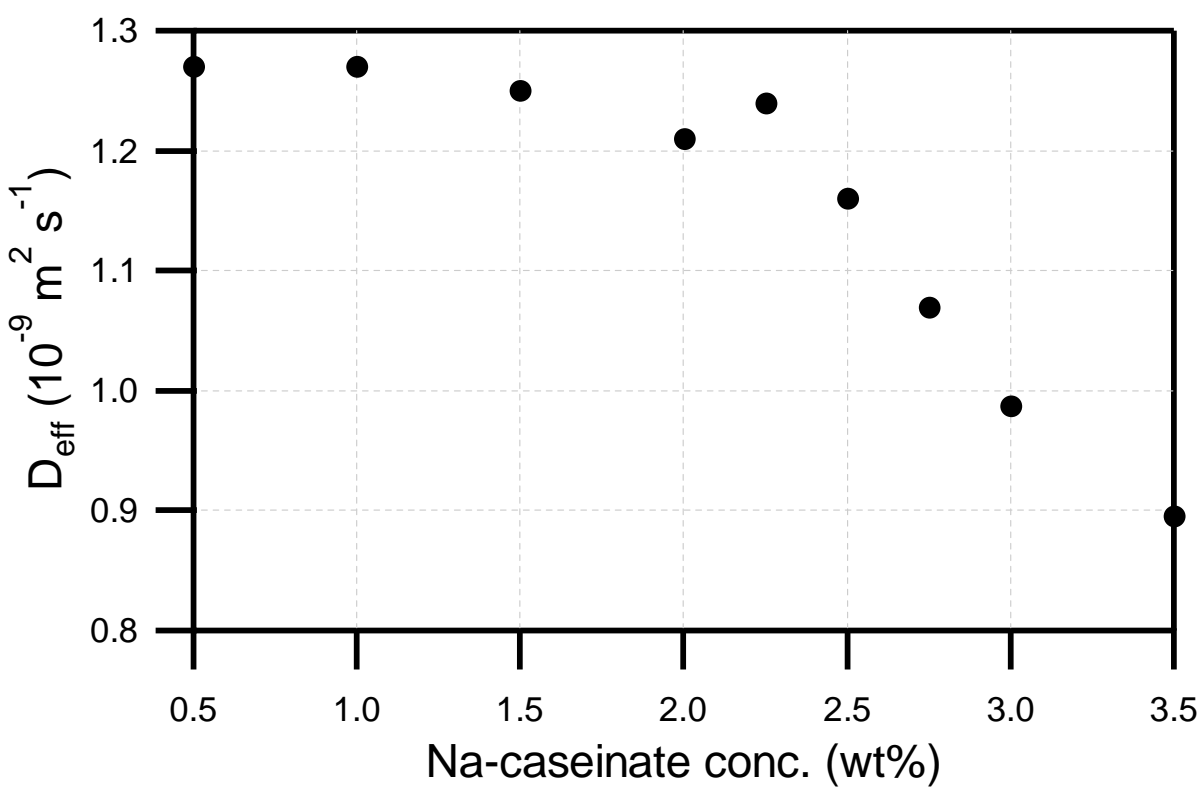

Figure 7.4: Diffusion coefficient of water as a function of increasing Na-caseinate concentration, $\Delta=20 \mathrm{~ms}$, for freshly prepared $50 \mathrm{wt} \%$ palm oil/Na-caseinate/water emulsions.

For the diffusion of oil droplets, the obtained attenuation data showed a multiexponential decay as was shown in Fig. 5.7. In Fig. 7.5 is shown the diffusion spectrum obtained by performing an inverse Laplace transformation on the attenuation data, here for a $50 \mathrm{wt} \%$ palm oil $/ 2 \mathrm{wt} \% \mathrm{Na}$-caseinate $/ 48 \mathrm{wt} \%$ water emulsion at $\Delta=200 \mathrm{~ms}$. The diffusion coefficients span from approximately $7 \times 10^{-14}$ to $7.7 \times 10^{-12} \mathrm{~m}^{2} \mathrm{~s}^{-1}$ compared with approximately $6.7 \times 10^{-14}$ to $1.7 \times 10^{-11} \mathrm{~m}^{2} \mathrm{~s}^{-1}$ for the soybean oil system (Fig. 5.8). We denote the slowest to fastest oil diffusion peaks as peaks 1 to 4 .

In Fig. 7.6 is shown the diffusion coefficient extracted at the maximum of each of the four oil diffusion peaks shown in Fig. 7.5 as a function of increasing observation time, $\Delta$, for $50 \mathrm{wt} \%$ palm oil $/ 2 \mathrm{wt} \% \mathrm{Na}$-caseinate $/ 48 \mathrm{wt} \%$ water emulsions following the same line of analysis as undertaken in Chapter 5. For peak 1, only $\Delta$ values up to a maximum of $800 \mathrm{~ms}$ could be used (longer $\Delta$ reaches the lower diffusion coefficient limit of the instrument). The diffusion coefficient decayed continuously with incrementing $\Delta$. The corresponding product of $D \cdot \Delta$ is seen to be constant; this coupled with the disappearance of this oil diffusion peak upon dilution confirm that peak 1 is due to the restricted diffusion of oil droplets in the bimodal droplet size distribution.

The diffusion coefficient for peak 2 also decreased continuously on increasing observation time with the cut off at $\Delta=800 \mathrm{~ms}$. For $\Delta>800 \mathrm{~ms}$, the diffusion coefficient remains approximately constant. The product of $D \cdot \Delta$ however increases on incrementing $\Delta$. 
Taking into consideration both $D$ vs. $\Delta$ and $D \cdot \Delta$ vs. $\Delta$ plots at $\Delta=200 \mathrm{~ms}$ (Fig. 7.6), $D$ and $D \cdot \Delta$ is in a decreasing and increasing trend, respectively. Hence, peak 2 reflects a combination of different types of diffusion, i.e., restricted and unrestricted diffusion of the oil droplets.

For peak 3, the measured diffusion coefficient appears to be constant for $\Delta<1000 \mathrm{~ms}$ and a decreasing function of $\Delta$ for longer times. On the other hand, the product of $D \cdot \Delta$ increases up to $\Delta=1000 \mathrm{~ms}$. On further increasing $\Delta, D \cdot \Delta$ is approximately constant. Hence, below $1000 \mathrm{~ms}$ unrestricted diffusion of the oil droplets is being probe and the motion becomes increasingly restricted at longer times.

The diffusion coefficient for peak 4 remains approximately constant whereas $D \cdot \Delta$ increased for all values of $\Delta$ used indicating that this fastest oil diffusion peak is due to unrestricted diffusion of the smallest oil droplets in the bimodal droplet size distribution. Again, we observed the disappearance of peak 4 upon dilution.

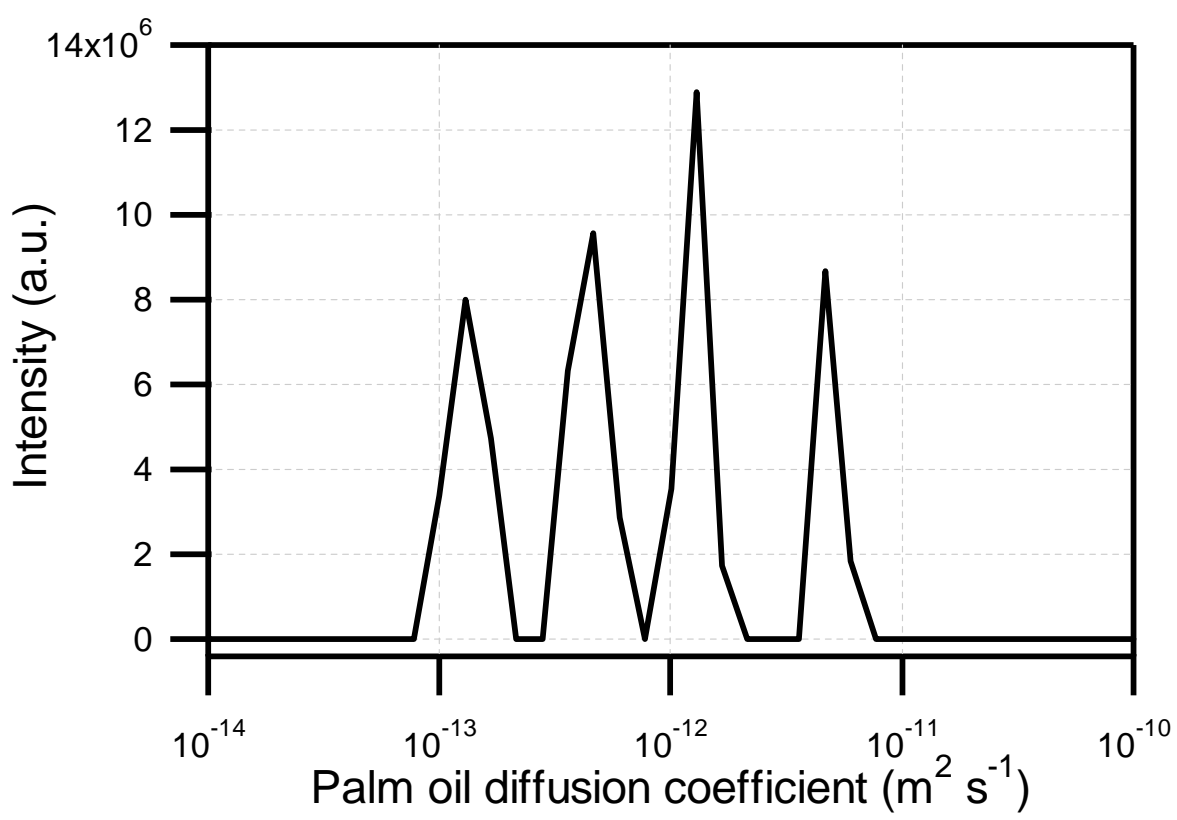

Figure 7.5: Palm oil diffusion coefficient distribution for $50 \mathrm{wt} \%$ palm oil $/ 2 \mathrm{wt} \% \mathrm{Na}$-caseinate $/ 48 \mathrm{wt} \%$ water emulsion, $\Delta=200 \mathrm{~ms}$. 

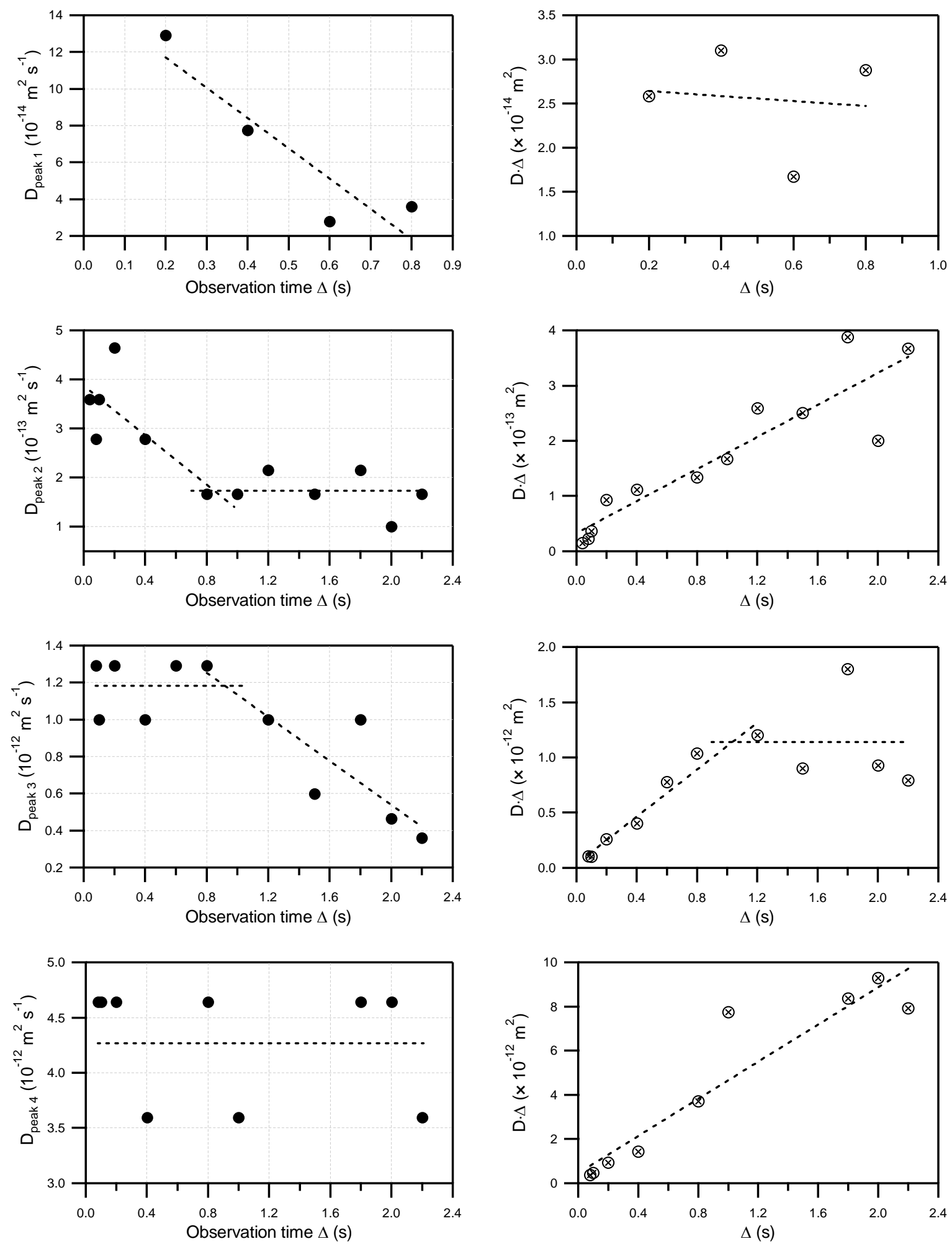

Figure 7.6: Palm oil diffusion coefficients as a function of increasing observation time, $\Delta$, for $50 \mathrm{wt} \% \mathrm{palm}$ oil $/ 2$ $\mathrm{wt} \% \mathrm{Na}$-caseinate $/ 48 \mathrm{wt} \%$ water emulsion obtained using an inverse Laplace transformation. Each figure represents the diffusion coefficient of one of the four different oil diffusion peaks shown in Fig. 7.5 at its maximum with their corresponding product of $D \cdot \Delta$ shown on the right hand side. 
The effect of palm oil and Na-caseinate concentration on the slowest oil diffusion peak seen in Fig. 7.5, due to the restricted diffusion of the oil droplets, was investigated. Since the relative intensity of pure restricted diffusion of the oil droplets on incrementing oil concentration was investigated for soybean oil $/ 2 \mathrm{wt} \% \mathrm{Na}$-caseinate/water emulsions (see Fig. 5.11 (b)), it would be interesting to compare this with that of palm oil system. As is observed in Fig. 7.7 (a), the relative intensity of the slowest oil diffusion peak extracted from the inverse Laplace transformation also increases linearly on increasing palm oil concentration. In Fig. 7.7 (b) is shown the relative intensity of the same oil diffusion peak on increasing Nacaseinate concentration for $50 \mathrm{wt} \%$ palm oil/ $\mathrm{Na}$-caseinate/water emulsions. At Na-caseinate concentration $\leq 2 \mathrm{wt} \%$ the relative intensity of the slowest oil diffusion peak is constant followed by a sudden jump at higher Na-caseinate concentration before becoming near constant again at approximately $2.75 \mathrm{wt} \%$ of Na-caseinate. These observed transition coincide with the variation of macroscopic stability in the phase diagram on traversing from domain I to II to III in Chapter 4.

\subsubsection{Rheology}

The form of the strain amplitude dependence of the storage and loss moduli, i.e., $G^{\prime}$ and $G^{\prime \prime}$ for all freshly prepared palm oil/Na-caseinate/water emulsions is very similar to that presented in Fig. 5.13. Both $G^{\prime}$ and $G^{\prime \prime}$ are parallel to each other at small strains with $G^{\prime}$ being larger than $G^{\prime \prime}$. This linear regime is separated from the non-linear regime by a cross over at the strain amplitude $>10 \%$. Beyond the cross over point, $G^{\prime \prime}$ lies above $G^{\prime}$.

In Fig. 7.8 is shown the response of palm oil $/ 2 \mathrm{wt} \% \mathrm{Na}$-caseinate/water emulsions in the linear regime. $G^{\prime}$ is always greater than $G^{\prime \prime}$ and they are separated by about an order of magnitude. Moreover, they are unaffected by incrementing palm oil concentration. Linear viscoelastic measurements at a fixed palm oil concentration, in this case $50 \mathrm{wt} \%$ but increasing Na-caseinate concentration is shown in Fig. 7.9. Over the applied frequency range, $G^{\prime}$ and $G^{\prime \prime}$ are also separated by about an order of magnitude but here they increase on increasing Na-caseinate concentration.

The steady state flow curves for $53 \mathrm{wt} \%$ palm oil $/ 2.5,3.5$ and $5 \mathrm{wt} \% \mathrm{Na}-$ caseinate/water emulsions at different shearing times are given in Fig. 7.10. The four rheological responses observed are very similar to those of the soybean oil system, corresponding to successive deformation, shear-thinning ${ }^{4,5}$, shear banding and Newtonian 
behaviours as identified in Fig. 6.1. The stresses at which the four regions occur are increasing functions of Na-caseinate concentration. $\dot{\gamma}_{1}$ and $\dot{\gamma}_{2}$ values extracted from Fig. 7.10 are summarised in Table 7.1. These limiting shear rates span approximately two orders of magnitude, irrespective of palm oil and Na-caseinate concentration, and despite the macroscopic stability variation of the emulsions. No specific trend is observed for $\dot{\gamma}_{1}$ and $\dot{\gamma}_{2}$. This is identical to the soybean oil system.
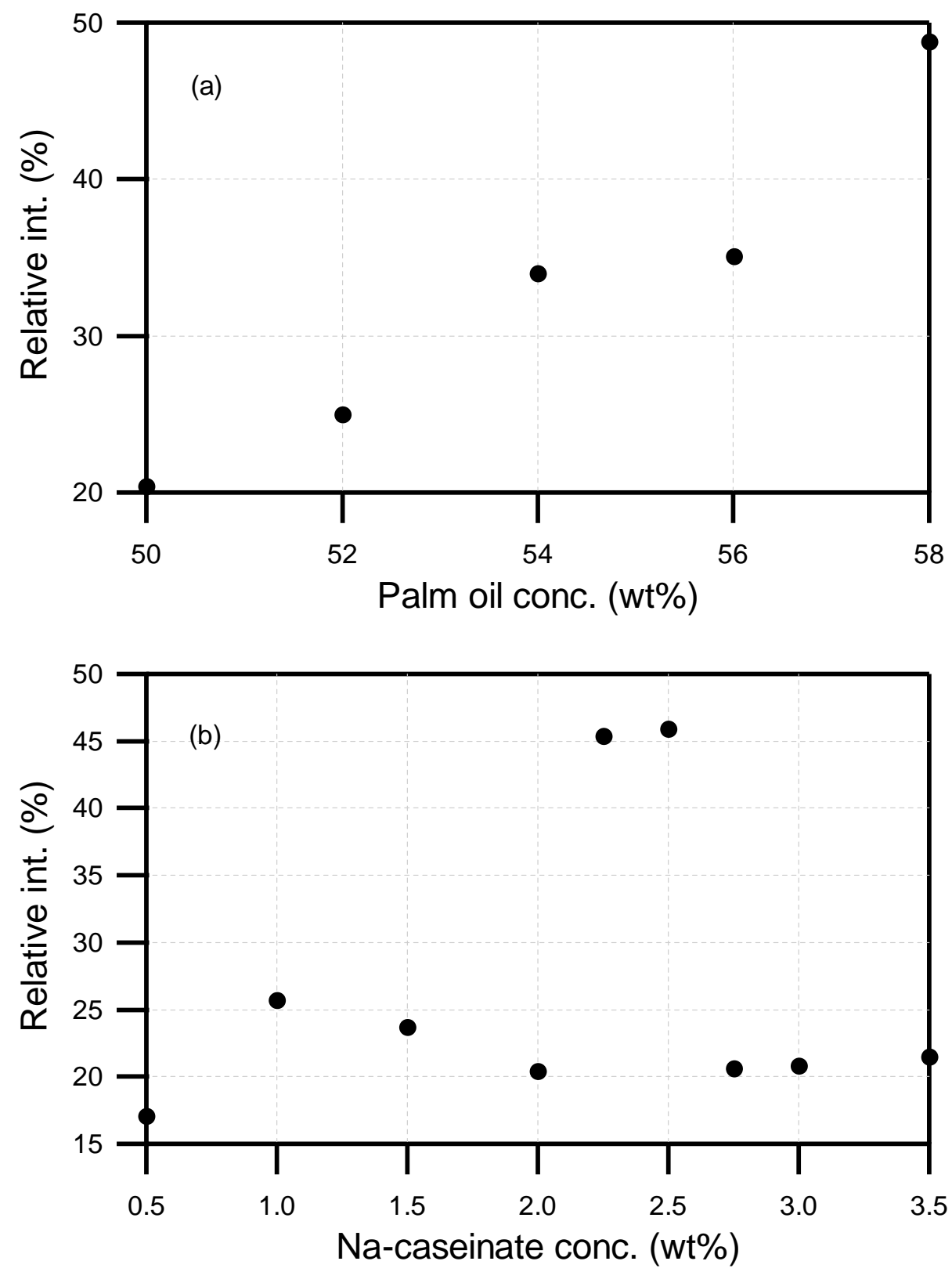

Figure 7.7: Relative intensity of the slowest oil diffusion peak (peak 1) as a function of increasing (a) palm oil concentration, for freshly prepared palm oil/2 wt\% Na-caseinate/water emulsion and (b) Na-caseinate concentration, for freshly prepared $50 \mathrm{wt} \%$ palm oil $/ \mathrm{Na}$-caseinate/water emulsions, $\Delta=200 \mathrm{~ms}$. 


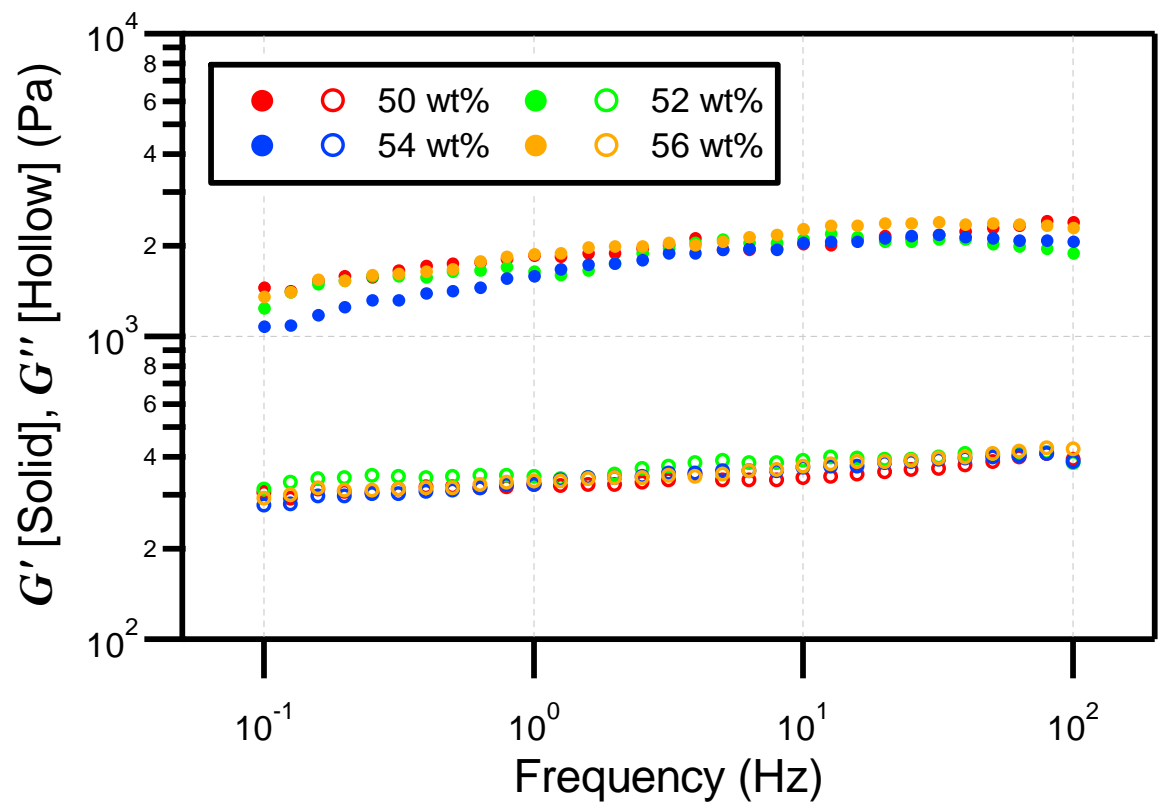

Figure 7.8: Log-log plot of the frequency dependence of $G^{\prime}$ and $G^{\prime \prime}$ at a strain amplitude of $2 \%$, for freshly prepared palm oil $/ 2.5 \mathrm{wt} \% \mathrm{Na}$-caseinate/water emulsions.

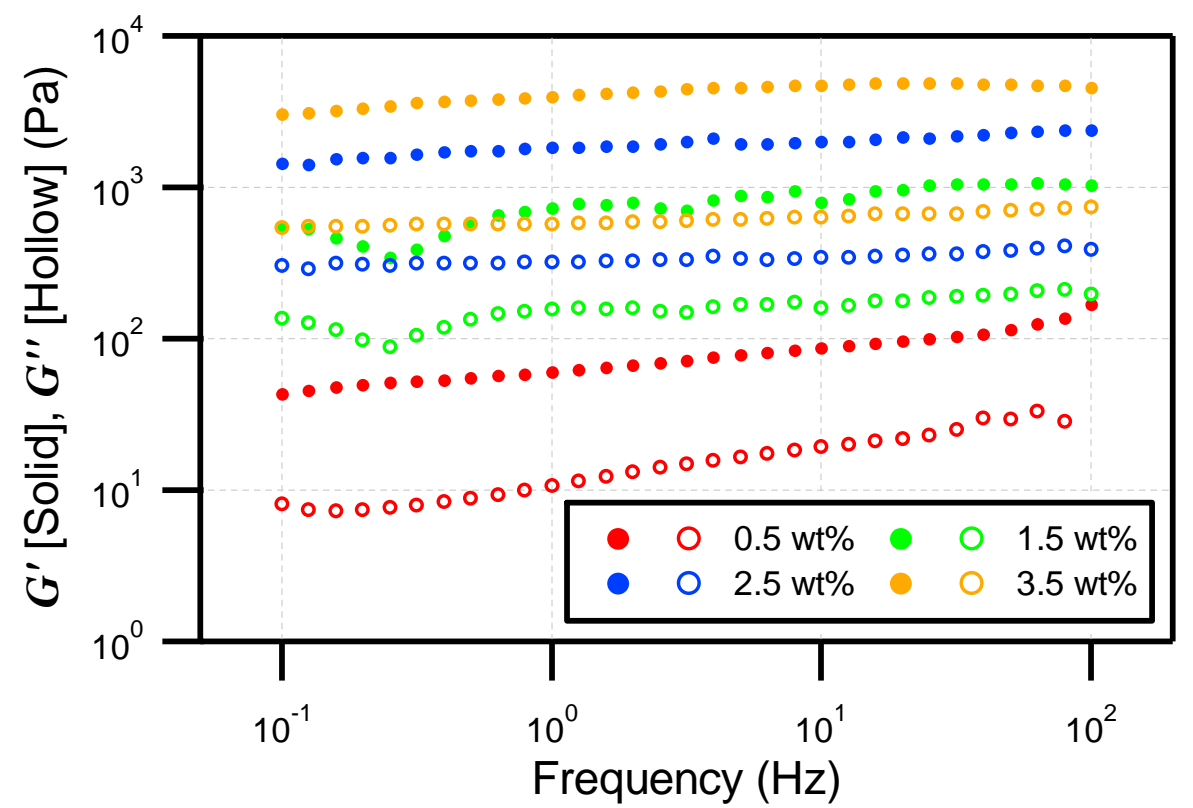

Figure 7.9: $\log -\log$ plot of the frequency dependence of $G^{\prime}$ and $G^{\prime \prime}$ at a strain amplitude of $2 \%$ on increasing $\mathrm{Na}$-caseinate concentration, for freshly prepared $50 \mathrm{wt} \% \mathrm{palm}$ oil/Na-caseinate/water emulsions.

\subsubsection{Cryo-SEM}

Fig. 7.11 represents the microstructure of $50 \mathrm{wt} \%$ palm oil/ $/ 0.5,2.5$ and $4 \mathrm{wt} \% \mathrm{Na}-$ caseinate/water emulsions. These emulsions encompass domains I, II and III in the phase diagram of the palm oil system (see Chapter 4). All micrographs show the polydispersity in 
the size of the oil droplets with water existing as the continuous phase throughout the system, correlating well with predictions from the light scattering and the PGSTE-NMR results. For the $0.5 \mathrm{wt} \% \mathrm{Na}$-caseinate system, it is clearly noticeable in Fig. 7.11 (a) that the oil droplets bridge with the neighbouring oil droplets leaving a narrower space after sublimation of water as compared to $2.5 \mathrm{wt} \%$ Na-caseinate system (e.g., see red line bounded domain in Fig. 7.11 (d)). The above bridging argument is supported by Fig. 7.11 (b). Considering the fracture plane, oil droplets are joined to each other leading to a loss in identity of single discrete droplets.

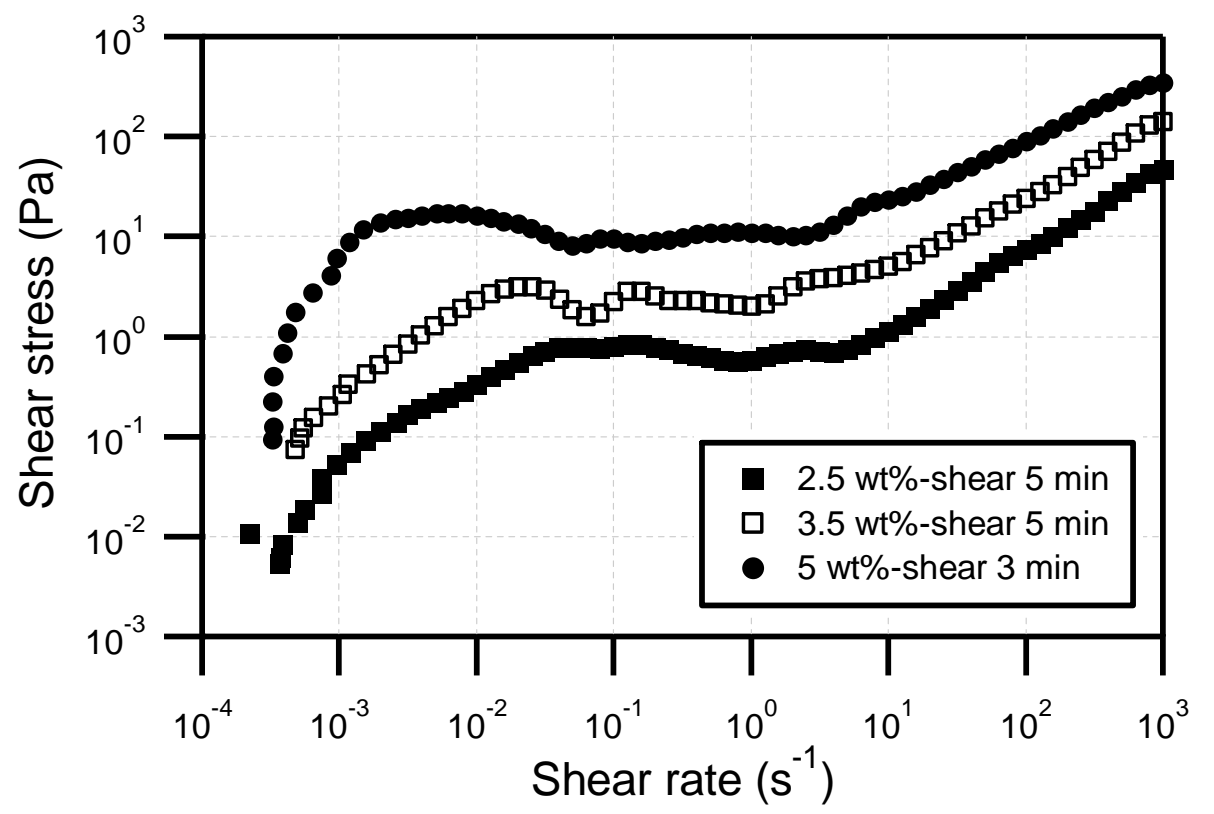

Figure 7.10: Stress vs. shear rate curves for $53 \mathrm{wt} \%$ palm oil/2.5, 3.5 and $5 \mathrm{wt} \%$ Na-caseinate/water emulsions during up-stress ramping: 1 min rest between each shear rate, from 0.0001 to $1000 \mathrm{~s}^{-1}$. Shearing times required to yield a steady state response varied with Na-caseinate concentration and are given in the legend. $\dot{\gamma}_{1}$ and $\dot{\gamma}_{2}$ values (see definition in Fig. 6.1) remain similar and span approximately two orders of magnitude on increasing Na-caseinate concentration.

Table 7.1: $\dot{\gamma}_{1}$ and $\dot{\gamma}_{2}$ values extracted from steady state flow curves for $53 \mathrm{wt} \%$ palm oil emulsions plotted in Fig. 7.10

\begin{tabular}{cccc}
\hline Critical shear rate $\left(\mathrm{s}^{-1}\right)$ & \multicolumn{3}{c}{ Na-caseinate concentration $(\mathrm{wt} \%)$} \\
& 2.5 & 3.5 & 5 \\
\hline$\dot{\gamma}_{1}$ & 0.0398 & 0.0316 & 0.0398 \\
$\dot{\gamma}_{2}$ & 3.98 & 1.26 & 2.51 \\
\hline
\end{tabular}



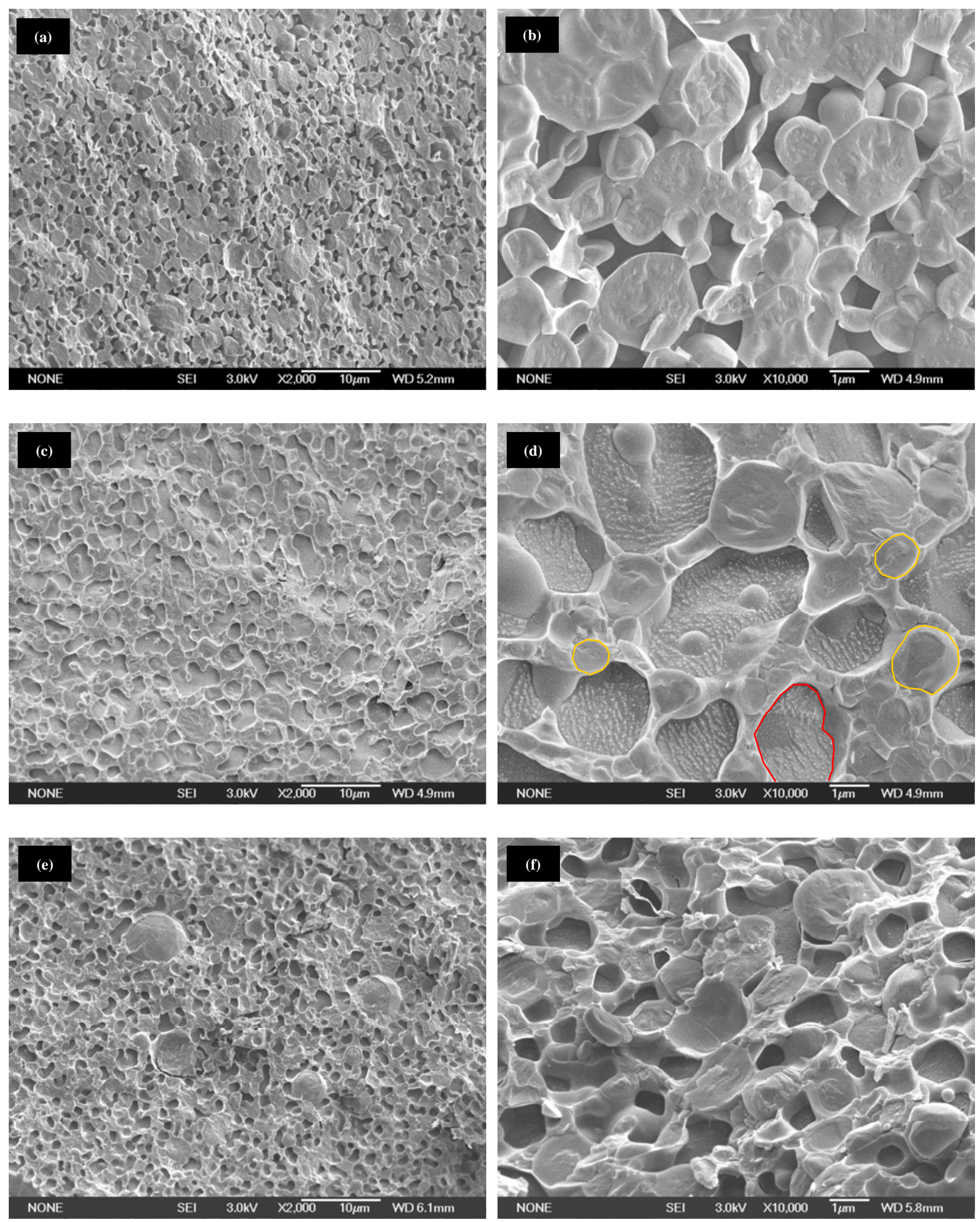

Figure 7.11: Cryo-SEM micrographs at 2000× magnification of freshly prepared $50 \mathrm{wt} \%$ palm oil/(a): 0.5, (c): 2.5 and (e): $4 \mathrm{wt} \%$ Na-caseinate/water emulsions after 30 minutes sublimation at $-110^{\circ} \mathrm{C}$ and their corresponding micrographs ((b), (d) and (f)) at 10000 $\times$ magnification. Examples of water and oil domains are indicated by red and yellow circles, respectively.

For $2.5 \mathrm{wt} \%$ Na-caseinate system, bridging is less obvious. In Fig. 7.11 (d), discrete oil droplets can be easily discriminated, e.g., those circled in yellow and the identity of 
individual oil droplets is largely maintained. Fig. 7.11 (e) presents a more or less spongy property where distant oil droplets seem to be connected in a network via a weaving method. The micrograph at $10000 \times$ magnification, i.e., Fig. 7.11 (f) further indicates the high porosity of $4 \mathrm{wt} \%$ Na-caseinate system. This is in contrast with 0.5 and $2.5 \mathrm{wt} \%$ Na-caseinate systems. In Fig. 7.11 (b) and (d), sublimation revealed the underneath structure where oil droplets are linked in three-dimension.

These microstructures of domain I, II and III in the palm oil system are directly comparable with those in the same domains in the soybean oil system.

\subsection{Discussion}

\subsubsection{PGSTE-NMR}

The analysis of the diffusion NMR data presented in Chapter 5, exemplified for $50 \mathrm{wt} \%$ soybean oil $/ 2 \mathrm{wt} \% \mathrm{Na}$-caseinate $/ 48 \mathrm{wt} \%$ water emulsion, should be comparable to emulsions in the palm oil system since both oils are triglycerides (mainly $\mathrm{C} 16$ and $\mathrm{C} 18$ fatty acids).

The application of Fourier transformation PGSE-NMR has recently been found in the studies of microemulsions. Skagerlind et al. ${ }^{6}$ and Stark et al. ${ }^{7}$ reported on the enzymatic hydrolysis of palm oil where microemulsions were used as a reaction media. The rate of reaction and the structure of the microemulsions were correlated with water content of the reaction medium via the water diffusion measurements. In particular, a decrease in water diffusion coefficient was believed to reflect a change from rod-like structure, to interconnected tubes to disconnecting water/oil spheres ${ }^{8}$.

In our case, the decrease of water diffusion coefficient on increasing palm oil (Fig. 7.3) and Na-caseinate (Fig. 7.4) concentration is attributed to enhanced interdroplet, water-droplet and/or unbound protein molecules or aggregates interactions. Thus, the travelling pathway of the water molecules is being restricted. It is this increase in tortuosity which decreases the measured diffusion coefficient for water.

Again, as for the soybean oil system the transition from domain I to II is evidenced by a switch from a constant water diffusion coefficient to a decreasing value on increasing Nacaseinate concentration. The transition from domain II to III is not readily distinguished, but is evident based on the obstruction factor. 
For diffusion measurements of oil droplets and molecules, each type of diffusion and its correlation to the experimental data will be discussed separately.

Palm oil has an unrestricted diffusion coefficient on the order of $10^{-10} \mathrm{~m}^{2} \mathrm{~s}^{-1}$. This fastest oil diffusion does not contribute to any measured diffusion coefficient of the palm oil emulsions since the oil droplets are sufficiently small so as to restrict the diffusion of the oil molecules for the values of $\Delta$ used. We will explain the observed diffusion coefficient distribution using the $50 \mathrm{wt} \%$ palm oil $/ 2 \mathrm{wt} \% \mathrm{Na}$-caseinate $/ 48 \mathrm{wt} \%$ water emulsion measured at $\Delta=200 \mathrm{~ms}$ as an example (see Fig. 7.5).

Diffusion peak 1. We see that in Fig. 7.6, $D \cdot \Delta$ vs. $\Delta$ and $D$ vs. $\Delta$ plots for peak 1 is constant and in a decreasing trend, respectively. Moreover, because of the disappearance of peak 1 upon dilution, it is obvious that this oil diffusion peak is due to oil droplets undergoing restricted diffusion among themselves. In Fig. 7.12 is shown the calculated mean square displacements determined using the measured diffusion coefficients at different observation times, $\Delta$. If we consider the calculated mean square displacement for $\Delta$ from 200 to $800 \mathrm{ms,}$ the shortest average mean distance between the oil droplets is $0.21 \pm 0.03 \mu \mathrm{m}$. This means that only oil droplets with a size less than the interdroplet spacing will be able to travel without any obstructions. Therefore, it is the oil droplets in the higher droplet mode of the bimodal distribution (i.e., $>0.24 \mu \mathrm{m}$, see Fig. 7.13) that are being restricted and hence contribute to the measured diffusion coefficient of peak 1 in Fig. 7.5.

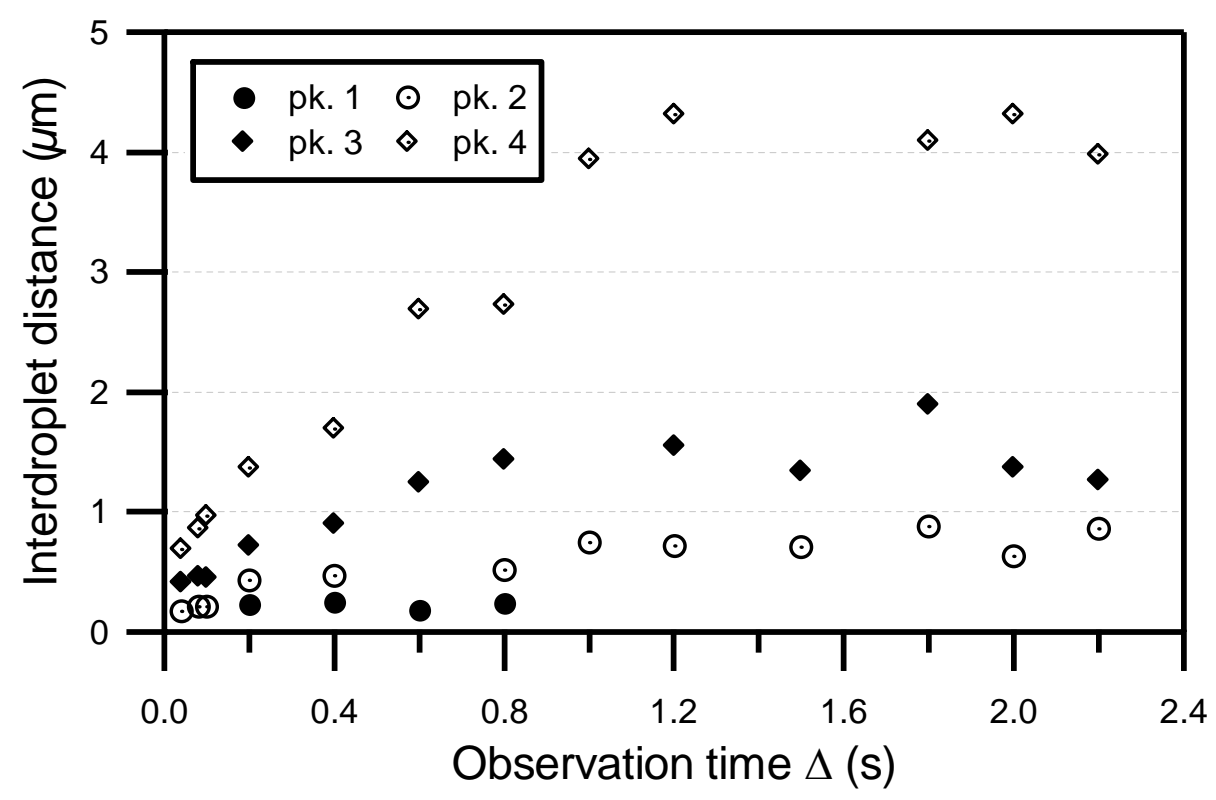

Figure 7.12: The calculated mean square displacement as a function of increasing observation time for diffusion coefficients of the four different oil diffusion peaks shown in Fig. 7.5, respectively. 


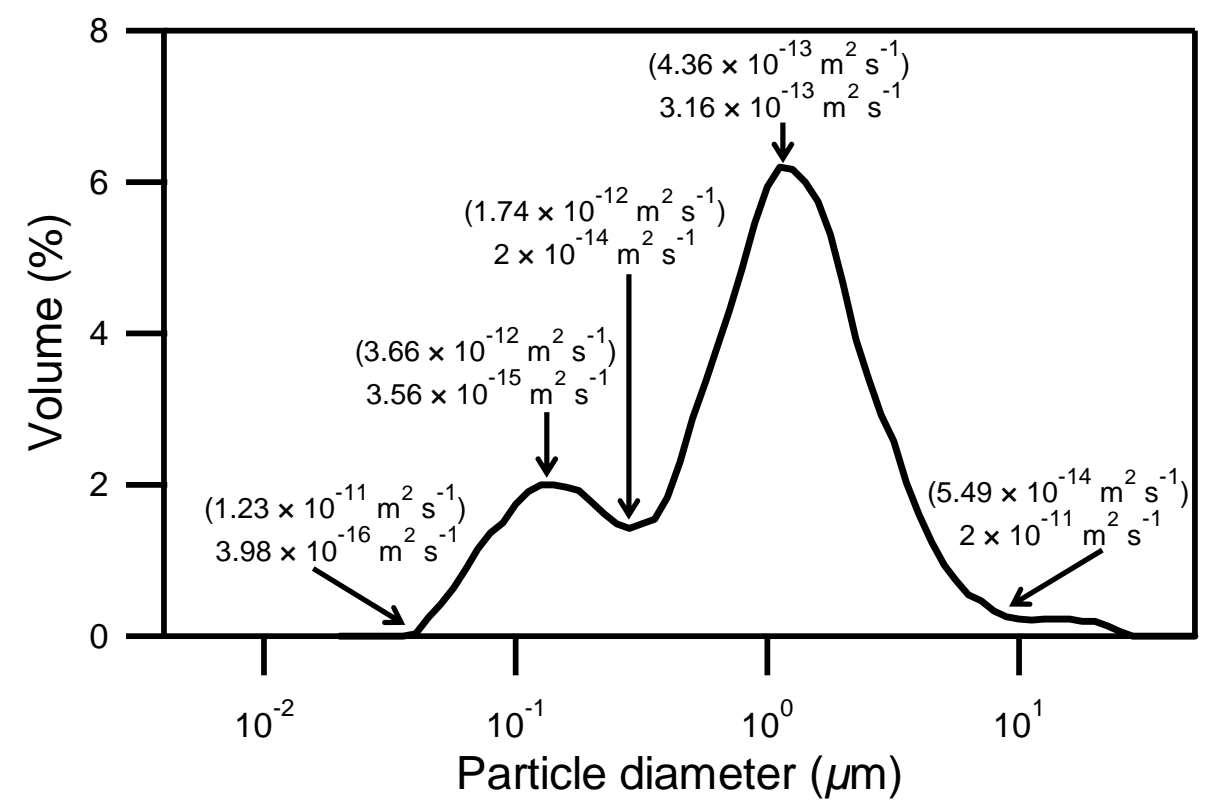

Figure 7.13: Expected (a) unrestricted diffusion coefficients of the oil droplets (in the brackets) and (b) diffusion coefficients of the oil molecules in the oil droplets (indicated by arrows) at five different oil droplet characteristic sizes of the bimodal distribution of freshly prepared $50 \mathrm{wt} \%$ palm oil $/ 2 \mathrm{wt} \% \mathrm{Na}$-caseinate $/ 48 \mathrm{wt} \%$ water emulsion, $\Delta=200 \mathrm{~ms}$.

At a fixed Na-caseinate concentration, upon incrementing the palm oil concentration, the amount of oil droplets formed will increase. Hence, the oil droplets are now more closely packed leading to a more pronounced restriction of the movement of oil droplets. This was indeed found to be the case, Fig. 7.7 (a). In addition, considering Fig. 7.7 (b) together with Fig. 5.12, two transitions occur at different Na-caseinate concentration for the two systems, correlating well with the corresponding phase boundaries distinguished in the phase diagrams between domains I and II and domains II and III hinting that the specific microstructures for emulsions formed with palm oil and soybean oil are in fact different. We note however that the variations are different in the two systems. This is believed to be due to their exact oil mix being different despite both oils being highly branched triglycerides (mainly $\mathrm{C} 16$ and C18 fatty acids).

Diffusion peak 2. Based on $D \cdot \Delta$ vs. $\Delta$ plot for peak 2 in Fig. 7.6, peak 2 is due to unrestricted diffusion of the oil droplets. Taking into consideration the calculated mean distance between the oil droplets for peak 2 at $\Delta=200 \mathrm{~ms}$, which is $0.43 \mu \mathrm{m}$ (see Fig. 7.12), it is clear that peak 2 in Fig. 7.5 corresponds to unrestricted diffusion of the oil droplets in the lower droplet mode of the bimodal distribution. On the other hand, $D$ vs. $\Delta$ plot is in a decreasing trend indicating some kind of restricted diffusion. Restricted diffusion between 
the oil droplets in the higher droplet mode of the bimodal distribution is a possible contribution to the measured diffusion coefficient of peak 2 in Fig. 7.5.

Moreover, restricted diffusion of the oil molecules in the larger oil droplets (i.e., oil droplets in the higher droplet mode of the bimodal distribution in Fig. 7.13) must not be ruled out. The calculated diffusion coefficients of the oil molecules in the oil droplets at $\Delta=200$ ms using equation 5.6 and the droplet size at five different points (guided by arrows) shown in Fig. 7.13 span from $10^{-16}$ to $10^{-11} \mathrm{~m}^{2} \mathrm{~s}^{-1}$. The measured diffusion coefficient of peak 2, which is $4.64 \times 10^{-13} \mathrm{~m}^{2} \mathrm{~s}^{-1}$, correlates well with the calculated diffusion coefficient of the oil molecules in the oil droplets using the fourth droplet size from the left in Fig. 7.13.

Upon dilution, this diffusion peak moves slightly to faster diffusion coefficients and the only two contributions are unrestricted diffusion of the oil droplets in the lower droplet mode and restricted diffusion of the oil molecules in the higher droplet mode of the bimodal distribution.

Diffusion peak 3. As can be seen from Fig. 7.6, for $\Delta \leq 1000 \mathrm{~ms}, D$ obtained is on the order of $10^{-12} \mathrm{~m}^{2} \mathrm{~s}^{-1}$ and the corresponding $D \cdot \Delta$ is in an increasing trend. These coupled with the average mean distance between the oil droplets which is $\sim 0.21 \mu \mathrm{m}$ suggest that unrestricted diffusion of the oil droplets in the lower droplet mode of the bimodal distribution in Fig. 7.13 is predominantly being measured. At longer observation time, i.e., for $\Delta>1000$ $\mathrm{ms}$, the motion of the oil droplets begins to be restricted and hence as was shown in Fig. 7.6, $D$ and $D \cdot \Delta$ decreases and remains approximately constant, respectively. Moreover, at $\Delta>$ $1000 \mathrm{~ms}, D$ measured for peak 3 span from $3.6 \times 10^{-13}$ to $1 \times 10^{-12} \mathrm{~m}^{2} \mathrm{~s}^{-1}$. Therefore, restricted diffusion of the oil molecules in the largest oil droplets (i.e., near the larger diameter tail of the higher droplet mode in Fig. 7.13) also contribute to the measured diffusion coefficient for peak 3 (see Fig. 7.13).

Returning to peak 3 in Fig. 7.5, as $D$ was measured as $1.29 \times 10^{-12} \mathrm{~m}^{2} \mathrm{~s}^{-1}$, this corresponds to unrestricted diffusion of the oil droplets with a size of $0.19 \mu \mathrm{m}$.

Diffusion peak 4. In contrast to peak 1 , for peak $4, D$ vs. $\Delta$ and $D \cdot \Delta$ vs. $\Delta$ plots in Fig. 7.6 is constant and in an increasing trend, respectively reflecting peak 4 in Fig. 7.5 is due to pure unrestricted diffusion of the oil droplets. All measured diffusion coefficients are on the order of $10^{-12} \mathrm{~m}^{2} \mathrm{~s}^{-1}$. Using the Stokes-Einstein relation (equation 3.5) and the droplet size at five different points in Fig. 7.13, the estimated unrestricted diffusion coefficients are given in Fig. 7.13 (see in the brackets). Mapping $D$ measured $\left(4.64 \times 10^{-12} \mathrm{~m}^{2} \mathrm{~s}^{-1}\right)$ with the values shown in the bracket, unrestricted diffusion of the smallest oil droplets in the lower 
droplet mode of the bimodal distribution in Fig. 7.13 is being measured.

Upon dilution, the disappearance of peak 4 in Fig. 7.5 could be attributed to two reasons: (1) upon dilution, the probability of having very small oil droplets in the NMR tube is reduced and thus the signal contribution is small. PGSTE-NMR technique and the inverse Laplace transformation and fitting are blind to the smallest oil droplets in the emulsion. (2) the signal contribution from unrestricted diffusion of the smallest oil droplets in the lower droplet mode of the bimodal distribution is combined with those for peak 3 and appears as one diffusion peak.

\subsubsection{Rheology}

Studies on the linear rheological properties of palm oil-in-water emulsions as compared to other types of oils (oleic oil, hydrogenated and refined coconut oils) was conducted by Granger et al. ${ }^{9}$. They attributed the stability of emulsions to interfacial interactions among the oil phase, emulsifier fatty acids and the adsorbed protein. Hayati et al. found that by substituting a certain amount of soybean oil to palm kernel olein caused an alteration of the linear rheology and hence the stability of the evaluated emulsions ${ }^{10}$.

From the data shown in Fig. 7.8, the overlapping of both $G^{\prime}$ and $G^{\prime \prime}$ implies that the microstructure of the studied emulsions is independent of the palm oil concentration. That is, the microstructure is dominated and defined by the Na-caseinate concentration. As observed in Fig. 7.9, on increasing Na-caseinate concentration from 0.5 to $3.5 \mathrm{wt} \%, G^{\prime}$ (and also $G^{\prime \prime}$ ) increased by nearly two orders of magnitude suggesting a more elastic-like behaviour. Incrementing Na-caseinate concentration results in a decreased mobility of the oil droplets ${ }^{11}$ and an increase of the viscosity of the continuous phase ${ }^{12}$. All emulsions are still in an undisturbed state (i.e., the microstructure has not been altered) as $G^{\prime}$ and $G^{\prime \prime}$ are constant over the applied frequency range; we are still in the linear regime.

Reports on non-linear rheology of Na-caseinate emulsions using palm oil as the dispersed phase can be hardly found. Nonetheless, Abd El-Salam et al. studied the flow properties of emulsions prepared using different whey protein concentrates/palm oil ratios and homogenisation pressures ${ }^{13}$. Heating of these emulsions at $80^{\circ} \mathrm{C}$ for 5 or 15 minutes markedly increased their viscosity. However, the viscosity of the heated emulsions decreased irreversibly with the increase in shearing.

From the phase diagram shown in Fig. 4.4 three phase boundaries are met for $50 \mathrm{wt} \%$ 
palm oil concentration on increasing Na-caseinate concentration. However, the four evolving rheological responses given in Fig. 7.10 occur irrespective of the palm oil and Na-caseinate concentration, i.e., despite the phase variation of the emulsions. This means that the differences in the aggregated state of the proteins ${ }^{14}$, the differences in the structure of the interfacial domain ${ }^{15,16}$ and therefore the interdroplet interactions ${ }^{17}$ are not manifested in the observed steady state flow curve other than in setting the value of the stress response. While the studied emulsions might undergo macroscopic destabilisation over time, competition between ageing and rejuvenation is believed to be the origin in controlling the observed flow curve evolution.

The continual variation in ageing and rejuvenation of sheared emulsion droplets defines the shear stress over the range of shear rates investigated, though the local features and the underlying microstructure change are not evident in the measured flow curves. As is seen in Fig. 7.10, the shear stress is obviously sensitive to the concentration of Na-caseinate. Considering the stress plateau, for example, the onset of plateau is not dependent on oil volume fraction (Table 7.1) but the corresponding shear stress increases on incrementing of Na-caseinate concentration.

Referring to Fig. 6.1 for the assignment of the four rheological responses in Fig. 7.10, regions I is associated with yield stress behaviour. Whereas region II and IV correlate to the break up and then the complete destruction of the macroscopic domains of aggregated structure and/or oil droplets, respectively. In between the shear-thinning and Newtonian regions, a shear-banded state indicated by a stress plateau has also been observed in the palm oil system. The existence of a stress plateau signifies an unstable flow ${ }^{18}$ which intrinsically arises from the interdroplet interaction ${ }^{19,20}$. The reader is directed to Chapter 6 for a fuller discussion.

\subsubsection{Cryo-SEM}

In Fig. 7.11 is shown the electron micrographs of emulsions at three different phase boundaries in the phase diagram for the palm oil system. In concentrated emulsions, the oil droplets are crowded together causing the interaction between the oil droplets to become important $^{21}$. For example, in our case with emulsions consisting of $0.5 \mathrm{wt} \% \mathrm{Na}$-caseinate, i.e., emulsions of domain I in the phase diagram, bridging flocculation is evident in Fig. 7.11

(a) and (b). Bridging flocculation occurs at low protein/oil ratios where the adsorbed protein 
tends to be shared resulting in the oil droplets bridging with adjacent droplets ${ }^{17}$ and hence the emulsions destabilise within 1 day. The microstructure difference between the soybean oil and palm oil systems hinted by the oil diffusion data (see Fig. 5.12 and Fig. 7.7) is evident in Fig. 7.11 (b) when comparing the micrograph with that of soybean oil system, i.e., Fig. 5.17 (a).

The $2.5 \mathrm{wt} \%$ Na-caseinate system (domain II) supports the above argument where the oil droplets are seen to be squeezed together leaving a large water domain. Nevertheless, as is shown in Fig. 7.11 (d), the oil droplets are discrete and easy-detectable. This is due to the system having sufficient protein available to cover the oil droplet surfaces ${ }^{16,22-25}$, emulsion stability is greatly enhanced.

On further increasing the Na-caseinate concentration up to $4 \mathrm{wt} \%$ (domain III in the phase diagram), flocculation is aided by the excess of unbound protein molecules or aggregates which are believed to create a cross-linked three-dimensional network ${ }^{17,26-28}$ as is presented in Fig. 7.11 (e) and (f) and was previously discussed in Chapter 3, resulting in a reduction in emulsion stability. The microstructure shown in Fig. 7.11 (e) and (f) appear to be much more similar with that observed in Fig. 5.17 (c). Gohtani et al. have reported the microstructure of monodispersed oil-in-water emulsion gel using cryo-SEM. The electron micrographs revealed that oil droplets are aggregated in the emulsion gel and that the gel has some void spaces between the gel network and the oil droplet aggregate ${ }^{29}$. This observation has similarity with our finding.

While we incorporated sublimation of water to reveal the network formation in the emulsions containing high Na-caseinate concentration, Ruis et al. studied the formation and collapse of the initial network (due to excess of Na-caseinate at neutral $\mathrm{pH}$ or acidification) of palmfat/Na-caseinate/water emulsions over time using diffusing wave spectroscopy (DWS) ${ }^{30}$. The emulsion droplets in an emulsion with and without an excess of Na-caseinate formed a network of oil droplets at neutral $\mathrm{pH}$ and upon acidification, respectively. Upon acidification of the earlier one, the initial network of oil droplets fell apart and eventually a network of Nacaseinate in which the oil droplets were embedded, was formed. Marumaya et al. on the other hand observed the deterioration of hardening palm oil during storage at different temperature under scanning electron microscopy ${ }^{31}$. To the best of our knowledge, the application of cryo-SEM on the study of Na-caseinate emulsion using palm oil as the dispersed phase has not been reported in the literature. 


\subsection{Conclusions}

Emulsions formed in domains I, II and III are oil-in-water emulsions with similar droplet size distributions upon preparation. PGSTE-NMR results show that at a fixed Na-caseinate concentration but increasing palm oil concentration, the diffusion coefficient of water decreases. This is due to the enhanced packing of the oil droplets and hence the tortuosity in the water diffusion pathway is being increasingly restricted. At a fixed palm oil concentration, the water diffusion coefficient remained constant followed by a decrease at $\sim 2.25 \mathrm{wt} \%$ of Na-caseinate. This transition corresponds to the observed macroscopic stability change presented in the phase diagram, i.e., from domain I to II (see Fig. 4.4).

For the palm oil diffusion, at a fixed Na-caseinate concentration, there is an increment in the relative intensity of the slowest oil diffusion peak that is due to pure restricted diffusion between oil droplets when the palm oil concentration is increased. At a fixed palm oil concentration, the relative intensity of the slowest oil diffusion peak remained unchanged, followed by a sudden jump before becoming nearly constant again at approximately 2.75 $\mathrm{wt} \%$ of Na-caseinate. The three transitions observed are in good agreement with the phase boundaries observed in the phase diagram, i.e., from domain I to II to III.

At a fixed Na-caseinate concentration, the emulsions showed identical viscoelastic properties on increasing palm oil concentration indicating the non-linear rheology of the emulsions is oil independent. However, at a fixed palm oil concentration, $G^{\prime}$ and $G^{\prime \prime}$ increased on increasing Na-caseinate concentration. The emulsions are predominantly solidlike. Shear banding is observed in the steady state flow curve irrespective of palm oil and Na-caseinate concentration, and despite the macroscopic stability variation of the emulsions. Cryo-SEM micrographs provide evident proof for the observed variation of macroscopic stability in the phase diagram of this palm oil system in Chapter 4. 


\subsection{References}

1. D. P. J. Moran, in PORIM Technology Paper (Palm Oil Research Institute of Malaysia (PORIM), 1993).

2. M. C. Chow and C. C. Ho, J. American Oil Chemists' Soc. 73, 47-53 (1996).

3. K. Ahmad, C. C. Ho, W. K. Fong and D. Toji, J. Colloid Interface Sci. 181, 595-604 (1996).

4. F. D. Cruz, F. Chevoir, D. Bonn and P. Coussot, Phys. Rev. E 66, 051305-051307 (2002).

5. J. N. Roux and G. Combe, C.R. Physique 3, 131-140 (2002).

6. P. Skagerlind and K. Holmberg, J. Dispersion Sci. Tech. 15, 317-332 (1994).

7. M.-B. Stark, P. Skagerlind, K. Holmberg and J. Carlfors, Colloid Polym. Sci. 268, 384-388 (1990).

8. S. Chen, D. Evans, B. Ninham, D. Mitchell, F. Blum and S. Pickup, J Phys. Chem. 90, 842-847 (1986).

9. C. Granger, P. Barey, P. Veschambre and M. Cansell, Colloids Surfs. B 42, 235-243 (2005).

10. I. Nor Hayati, Y. bin Che Man, C. P. Tan and I. Nor Aini, Food Res. Int. 40, 10511061 (2007).

11. A. J. Vasbinder, P. J. J. M. van Mil, A. Bot and K. G. de Kruif, Colloids Surfs. B 21, 245-250 (2001).

12. H. G. M. Ruis, P. Venema and E. van der Linden, Food Hydrocolloids 21, 545-554 (2007).

13. M. H. Abd El-Salam, S. El-Shibiny and H. M. El-Etriby, Egy. J. Dairy Sci. 22, 131143 (1994).

14. D. G. Dalgleish, Food Res. Int. 29, 541-547 (1996).

15. D. F. Evans and H. Wennerström, The colloidal domain: where physics, chemistry, biology and technology meet. (Wiley VCH, New York, 1994).

16. Y. Fang and D. G. Dalgleish, J. Colloid Interface Sci. 156, 329-334 (1993).

17. E. Dickinson, M. Golding and J. W. P. Malcolm, J. Colloid Interface Sci. 185, 515529 (1997).

18. J. Yerushalmi, S. Katz and R. Shinnar, Chem. Eng. Sci. 25, 1891-1902 (1970). 
19. J. W. Goodwin and W. B. Russel, Curr. Opin. Colloid Interface Sci. 2, 409-410 (1997).

20. L. Bécu, S. Manneville and A. Colin, Phys. Rev. Lett. 96, 138302-138305 (2006).

21. R. Pal, Chem. Eng. J. 67, 37-44 (1997).

22. E. Dickinson, J. Chem. Soc., Faraday Trans. 88, 2973-2983 (1992).

23. E. Dickinson, E. W. Robson and G. Stainsby, J. Chem. Soc., Faraday Trans. 79, 29372952 (1983).

24. A. R. Mackie, J. Mingins and A. N. North, J. Chem. Soc., Faraday Trans. 87, 30433049 (1991).

25. D. G. Dalgleish, in Food macromolecules and colloids, edited by E. Dickinson and D. Lorient (Royal Society of Chemistry, Cambridge, 1995), pp. 23.

26. C. L. A. Berli, D. Quemada and A. Parker, Colloids Surfs. A 203, 11-20 (2002).

27. E. Dickinson, S. J. Radford and M. Golding, Food Hydrocolloids 17, 211-220 (2003).

28. S. J. Radford and E. Dickinson, Colloids Surfs. A 238, 71-81 (2004).

29. S. Gohtani, K.-H. Kim and Y. Yamano, in Hydrocolloids, edited by K. Nishinari (Elsevier Science B.V., Amsterdam, 2000), pp. 91-96.

30. H. G. M. Ruis, K. van Gruijthuijsen, P. Venema and E. van der Linden, Langmuir 23, 1007-1013 (2007)

31. T. Maruyama, I. Niiya, M. Tsukamoto, S. Tokairin and T. Matsumoto, Yukagaku 35, 656-658 (1986). 


\section{Chapter 8}

\section{Tetradecane Emulsions}

\subsection{Introduction}

The nature of the oil, often rendered through the alkane carbon number $(\mathrm{ACN})$, or the equivalent alkane carbon number (EACN) when the oil phase is not an alkane, is important in defining the physicochemical characteristics of emulsifier-oil-water systems ${ }^{1,2}$. For microemulsions, a decrease in ACN (the so-called formulation variable) has been reported to contribute to an increase in the interfacial interaction between the emulsifier and the oil ${ }^{3}$. In addition, the chain length and the oil structure is also found to affect the emulsion properties.

In this chapter, emulsions prepared with tetradecane as the dispersed phase will be discussed. This change in the chemical nature of the oil used induces a significant difference in the phase behaviour and stability of the emulsions formed. In the soybean oil and palm oil systems, dispersed droplet oil-in-water emulsions are realised. The phase space of these systems is very rich and the continuous phase characteristics are manipulated by altering the amount of Na-caseinate present. The self-assembled state of the Na-caseinate defines the macroscopic stability of the emulsions. Switching from the commercial oils to tetradecane changes the inherent hydrophobicity of the oil which modifies both the interfacial tension and the curvature of the interface between the oil and water domains, due to the oil now interacting with the Na-caseinate aggregates and individual proteins differently from both physical and chemical perspectives.

Tetradecane has poor solvent properties compared to soybean oil and palm oil. It is not soluble in water. Hence more energy input is required to produce an emulsion. Oil penetration of tetradecane is expected to be less effective which results in an increase in the bending elasticity ${ }^{4,5}$, leading to a thinner interfacial layer ${ }^{6,7}$. Excess energy input causes shear-induced flocculation during homogenisation as the balance between Gaussian curvature, interfacial tension and Gibbs elasticity is very delicate. In using tetradecane as the 
oil, the hydrophobic effect will be investigated further with respect to the stability and microstructure of Na-caseinate emulsions and information will be gained on how, through oil choice alone, emulsion characteristics can be controlled and manipulated.

\subsection{Results}

The phase diagram of this system has been discussed in Chapter 4. Within the phase space, two emulsion domains are distinguished plus a gel-like paste. All have water as the continuous phase. For what follows, only results for the study of emulsions in domain I will be presented unless specified otherwise.

\subsubsection{Laser Diffraction Particle Sizing}

The droplet size distributions of freshly prepared tetradecane $/ 50 \mathrm{wt} \%$ Na-caseinate/water emulsions in domain I of the phase diagram (see Fig. 4.6) were determined by static light scattering. The distributions, shown in Fig. 8.1, for all samples exhibit a similar lognormal shape. As was reported for the soybean oil and palm oil systems, the distribution for the tetradecane system is bimodal and does not significantly vary on increasing Na-caseinate concentration. Hence, a near identical droplet size distribution exists despite, for example, the macroscopic stability changing dramatically in each of the three systems (i.e., soybean oil, palm oil and tetradecane systems).

From Fig. 8.1, the oil droplet diameters of the emulsions formed measure from $\sim 0.04$ to $5.64 \mu \mathrm{m}$. The values of $D[3,2]$ and $D[4,3]$ span from 0.48 to $0.51 \mu \mathrm{m}$ and from 1.78 to 1.93 $\mu \mathrm{m}$, respectively. The two droplet modes are $\sim 0.14$ and $\sim 1.59 \mu \mathrm{m}$, respectively. A few large oil droplets and air bubbles created during emulsion formulation manifest themselves as a third droplet mode centred around $8 \mu \mathrm{m}$ in the distribution.

In Fig. 8.2 are shown the droplet size distributions of freshly prepared tetradecane/2 $\mathrm{wt} \% \mathrm{Na}$-caseinate/water emulsions for a fixed Na-caseinate concentration upon increasing tetradecane concentration. The distributions are, as described above, essentially bimodal. The oil droplet diameters measure from $\sim 0.04$ to $5.64 \mu \mathrm{m}$. $D[3,2]$ and $D[4,3]$ range from 0.43 to $0.53 \mu \mathrm{m}$ and from 1.72 to $1.83 \mu \mathrm{m}$, respectively. The bimodal distribution corresponds to 0.13 and $1.50 \mu \mathrm{m}$ for lower and higher droplet modes, respectively. The third 
droplet mode that is most likely due to the existence of a few large oil droplets is observed at $\sim 8 \mu \mathrm{m}$.

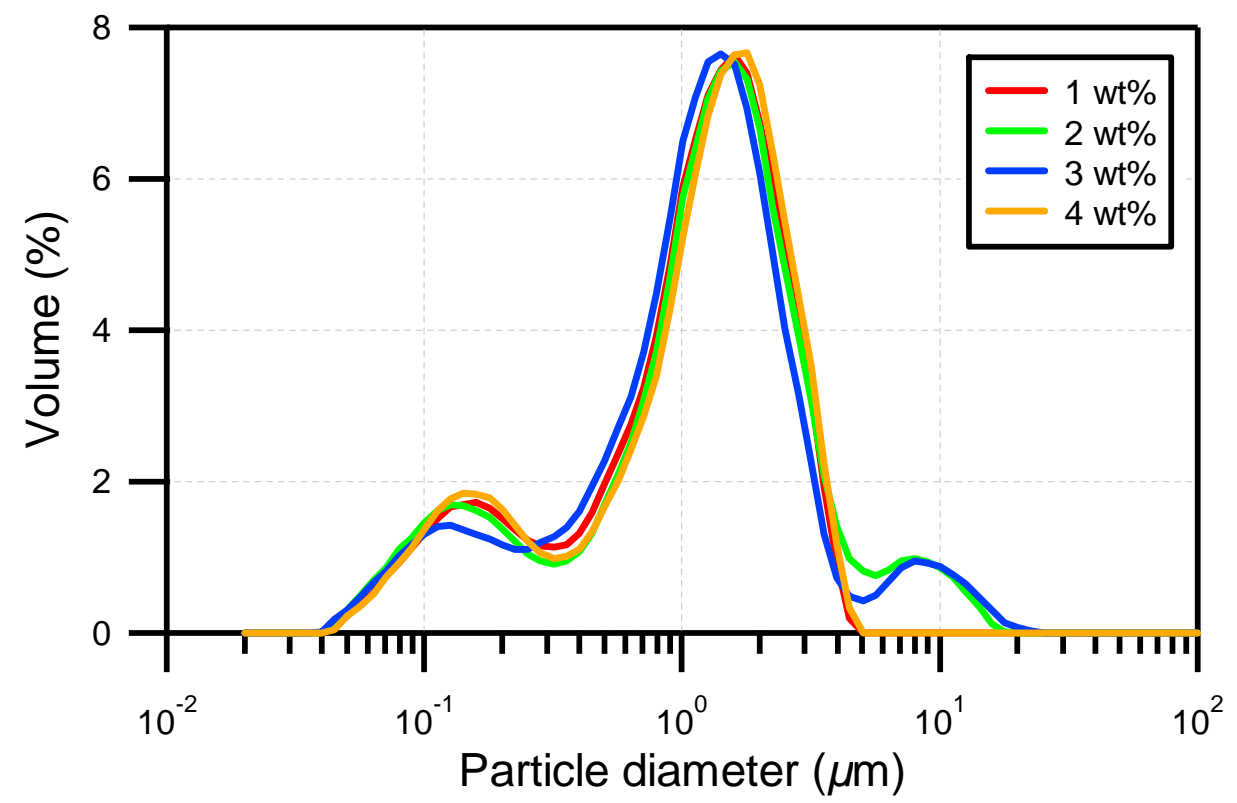

Figure 8.1: Droplet size distribution for freshly prepared $50 \mathrm{wt} \%$ tetradecane/Na-caseinate/water emulsions with $1,2,3$ and $4 \mathrm{wt} \%$ Na-caseinate.

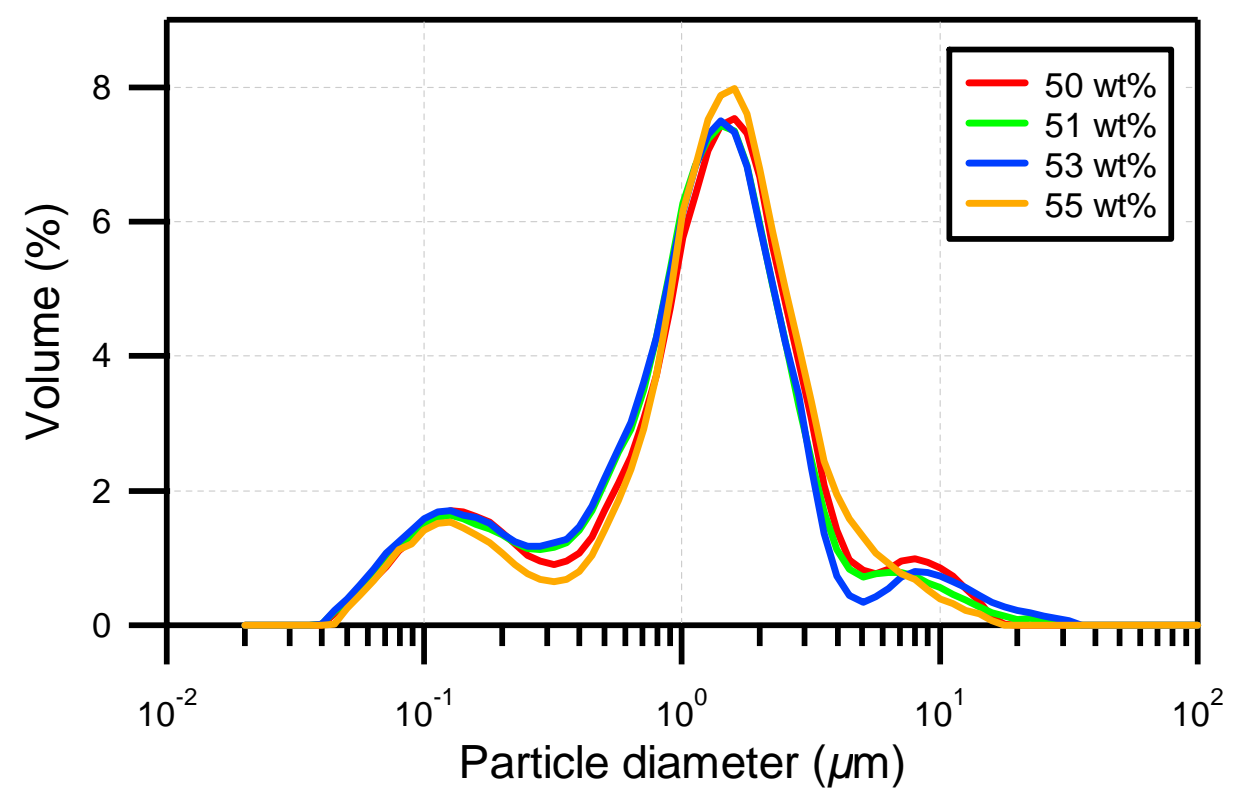

Figure 8.2: Droplet size distributions for freshly prepared tetradecane $/ 2 \mathrm{wt} \%$ Na-caseinate/water emulsions with $50,51,53$ and $55 \mathrm{wt} \%$ tetradecane. 


\subsubsection{PGSTE-NMR}

As in the case of soybean oil and palm oil systems, the proton signals for Na-caseinate also overlap with those of tetradecane. Hence, only results for the oil and water domains will be reported.

The attenuation data of water diffusion exhibit a single exponential decay. In Fig. 8.3 are shown the diffusion coefficients obtained for water for an observation time, $\Delta$ of $20 \mathrm{~ms}$ as a function of tetradecane concentration for tetradecane/1, $2 \mathrm{wt} \%$ Na-caseinate/water emulsions. We note that at higher Na-caseinate and tetradecane concentrations, the viscosity of the emulsions is greatly increased and it was not possible to fill the NMR tube. The diffusion coefficient of water is reduced on incrementing tetradecane concentration irrespective of Na-caseinate concentration. For the system with $1 \mathrm{wt} \% \mathrm{Na}$-caseinate, the measured water diffusion coefficient decreases from 1.22 to $1.12 \times 10^{-9} \mathrm{~m}^{2} \mathrm{~s}^{-1}$, while in the 2 $\mathrm{wt} \% \mathrm{Na}$-caseinate system it changes from 1.15 to $1.04 \times 10^{-9} \mathrm{~m}^{2} \mathrm{~s}^{-1}$. The decrease in the water diffusion coefficient when the water volume fraction is decreased can be ascribed to the obstruction effect due to the enhanced packing of the oil droplets, which hinders the diffusion path of the water as discussed previously ${ }^{8,9}$.

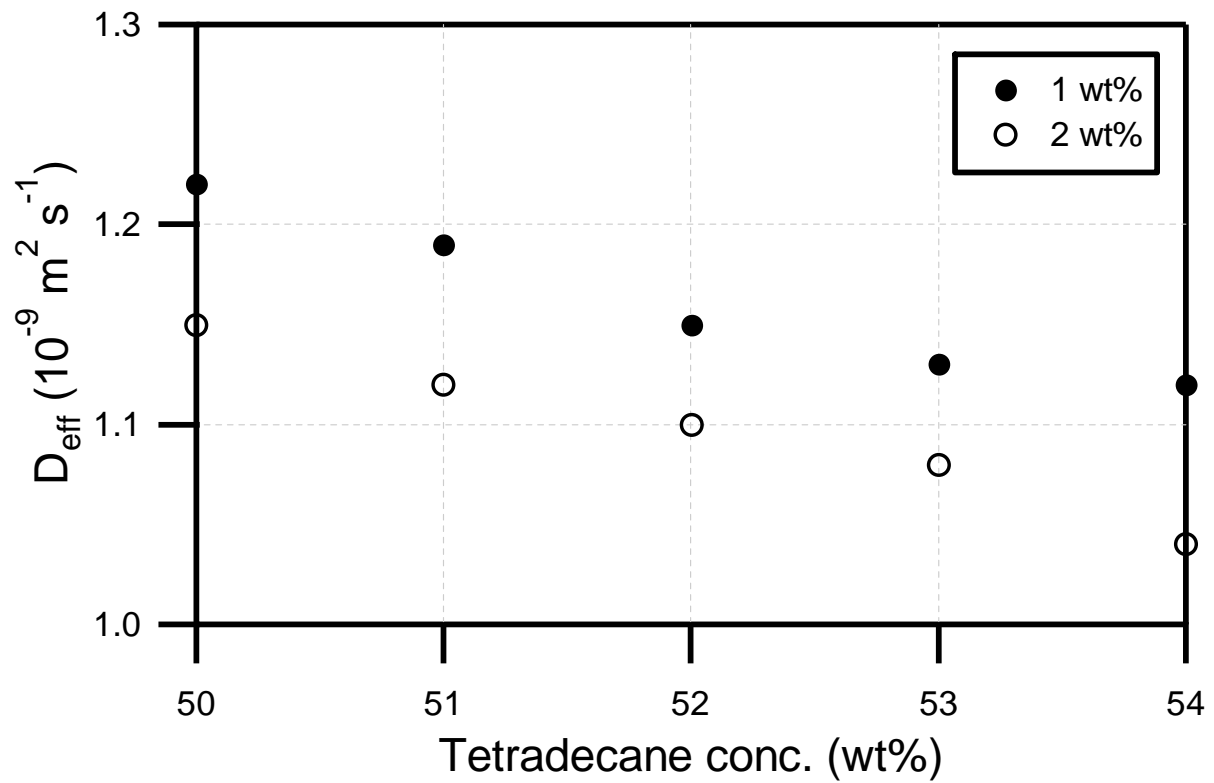

Figure 8.3: Diffusion coefficient of water as a function of increasing tetradecane concentration, $\Delta=20 \mathrm{~ms}$, for freshly prepared tetradecane/1, $2 \mathrm{wt} \% \mathrm{Na}$-caseinate/water emulsions.

The corresponding variation of the water diffusion coefficients as a function of 
increasing Na-caseinate concentration for $50 \mathrm{wt} \%$ tetradecane/Na-caseinate/water emulsions at $\Delta=20 \mathrm{~ms}$ is shown in Fig. 8.4. It is obvious that the diffusion coefficient of water decreases monotonically. This correlates to water diffusion of emulsions which have single phase behaviour, i.e., only one domain has been met in the phase diagram (see Fig. 4.6) on increasing the concentration of Na-caseinate from 0.5 to $2.5 \mathrm{wt} \%$. Again, due to high sample viscosity it was not possible to obtain data above $2.5 \mathrm{wt} \%$ Na-caseinate. As the measured water diffusion coefficients presented in Fig. 8.3 and Fig. 8.4 remain on the same order of magnitude as that of bulk water and for $\Delta$ values up to $200 \mathrm{~ms}$, this confirms that the emulsions studied are of the oil-in-water type and that the water undergoes unrestricted diffusion at all tetradecane and Na-caseinate concentrations.

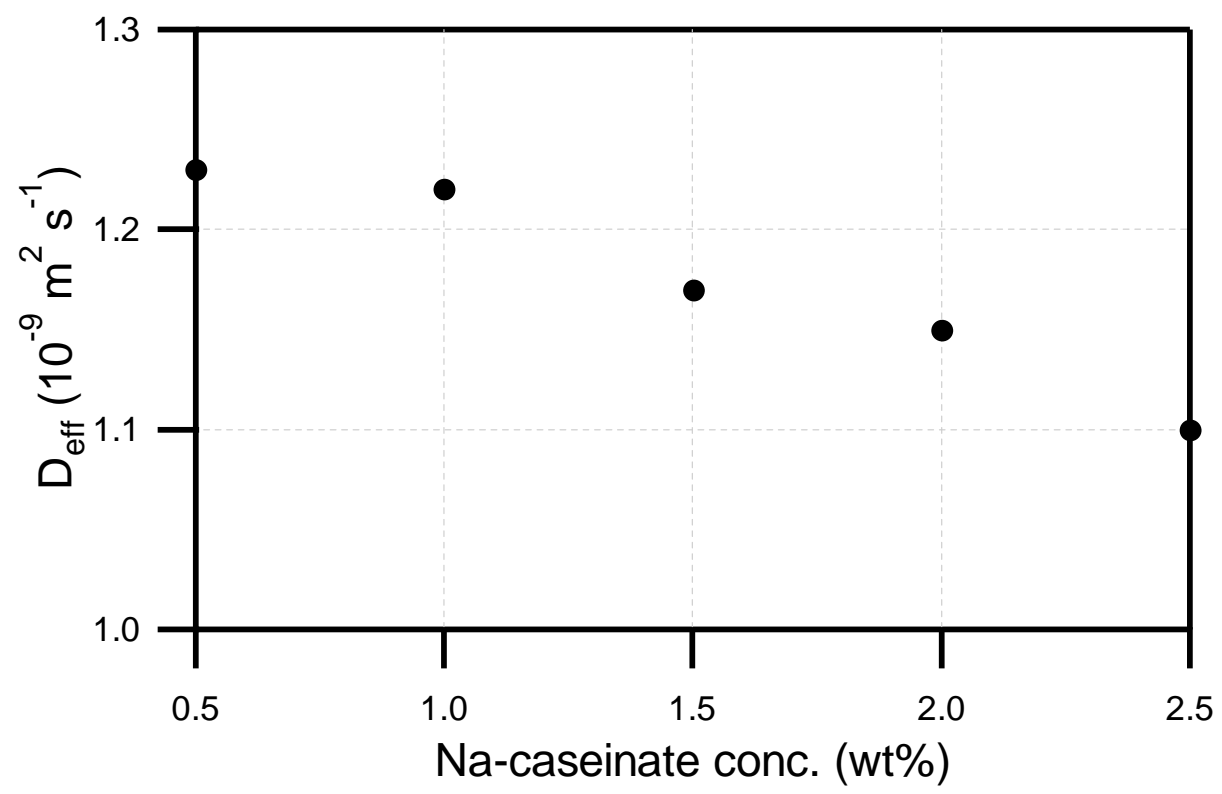

Figure 8.4: Diffusion coefficient of water as a function of increasing Na-caseinate concentration, $\Delta=20 \mathrm{~ms}$, for freshly prepared $50 \mathrm{wt} \%$ tetradecane/Na-caseinate/water emulsion.

As has been discussed in Chapter 5 and Chapter 7, the polydispersity and the existence of different types of oil-based diffusion in Na-caseinate emulsions should be mirrored by a multiexponential decay in the measured oil attenuation data as a function of gradient strength. In Fig. 8.5 is shown a multiexponential decay attenuation curve for a $50 \mathrm{wt} \%$ tetradecane/2 $\mathrm{wt} \% \mathrm{Na}$-caseinate $/ 48 \mathrm{wt} \%$ water emulsion acquired at $\Delta=200 \mathrm{~ms}$.

In Fig. 8.6 is shown the characteristic distribution of diffusion coefficients obtained at $\Delta$ $=100 \mathrm{~ms}$ via application of an inverse Laplace transform to the integrated data, i.e., a nonnegative least squares fit of the exponentially decaying signal, weighted by an additional 
regularisation function. Only two oil diffusion peaks exist in the distribution of diffusion coefficients for the undiluted emulsion with the diffusion coefficients ranging from approximately $6 \times 10^{-14}$ to $8 \times 10^{-12} \mathrm{~m}^{2} \mathrm{~s}^{-1}$. We denote the slowest and fastest oil diffusion peaks as peaks 1 and 2, respectively.

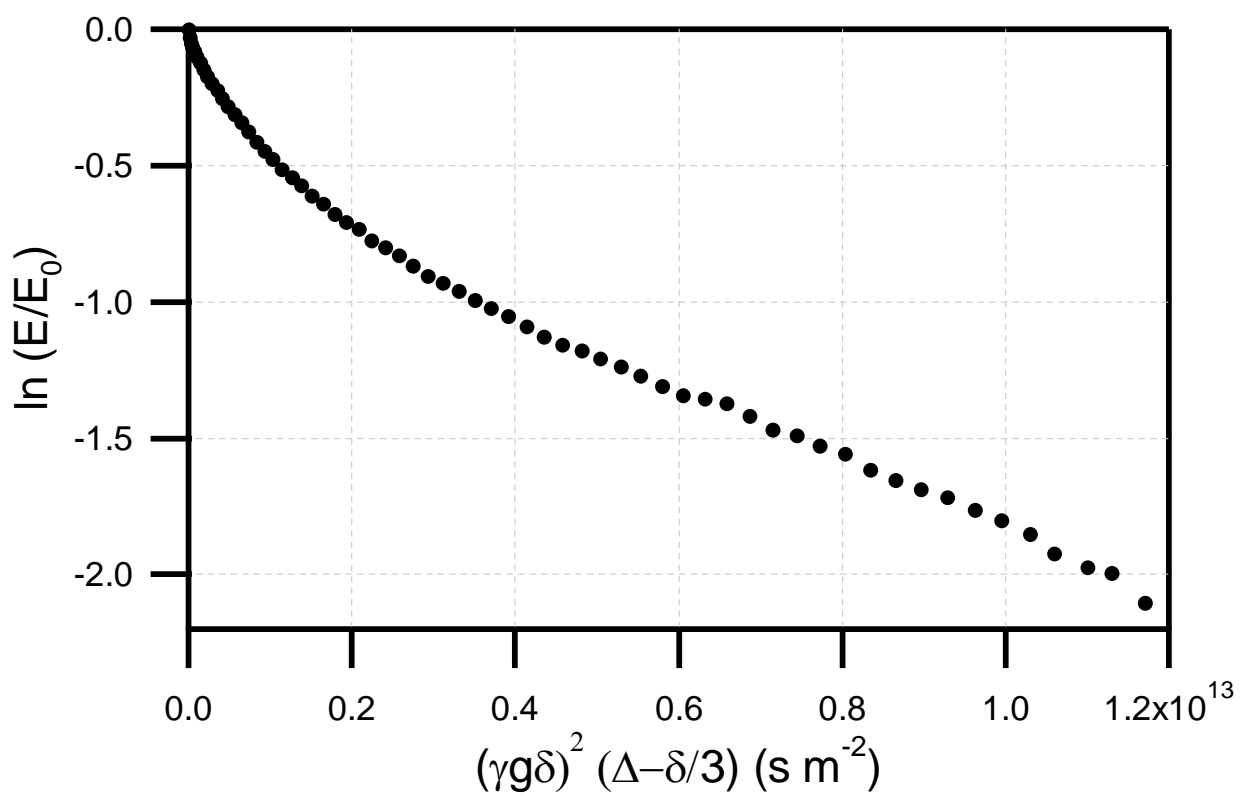

Figure 8.5: Echo attenuation plot for tetradecane diffusion in a freshly prepared $50 \mathrm{wt} \%$ tetradecane $/ 2 \mathrm{wt} \% \mathrm{Na}-$ caseinate $/ 48 \mathrm{wt} \%$ water emulsion, $\Delta=200 \mathrm{~ms}$.

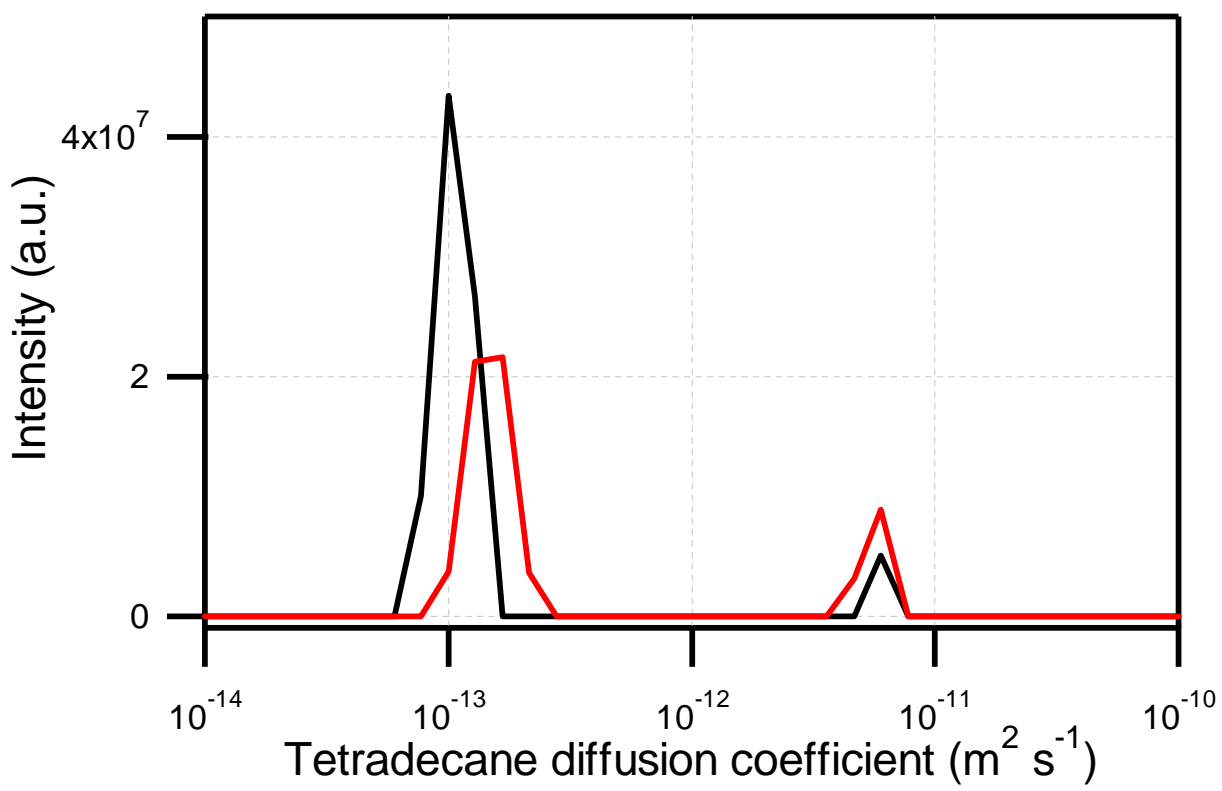

Figure 8.6: Tetradecane diffusion coefficient distribution for freshly prepared (black) and diluted (red) $50 \mathrm{wt} \%$ tetradecane $/ 2 \mathrm{wt} \%$ Na-caseinate/48 $\mathrm{wt} \%$ water emulsion obtained via application of an inverse Laplace transform ${ }^{10}$ to the attenuation data, $\Delta=100 \mathrm{~ms}$. 
Dilution was incorporated to in freshly prepared emulsions prior to measurement in order to resolve the oil diffusion peaks that are due to the restricted diffusion of the oil droplets. The data in red in Fig. 8.6 is the distribution of diffusion coefficients acquired for an emulsion to aqueous phosphate buffer volume ratio of 1:4. Upon dilution, the two oil diffusion peaks observed for the undiluted emulsion are still present and closer inspection reveals that the diffusion coefficients span from approximately $8 \times 10^{-14}$ to $8 \times 10^{-12} \mathrm{~m}^{2} \mathrm{~s}^{-1}$, a similar range to that obtained for the undiluted emulsion. This confirms that restricted diffusion of the oil droplets is absent from the measured diffusion distribution. This is in stark contrast to the situation for soybean oil and palm oil emulsions and at fast consideration is perplexing given that the droplet size distributions are nearly identical for all three systems. This will be addressed in discussion.

To account for the contributions of the different types of diffusion to each of the two oil diffusion peaks evident in Fig. 8.6, we studied the tetradecane diffusion coefficients as a function of incrementing $\Delta$. The results are shown in Fig. 8.7 where the diffusion coefficients are extracted at the maximum of each of the two oil diffusion peaks in the diffusion spectra obtained at different $\Delta$. The corresponding product of $D \cdot \Delta$ is presented on the right hand side of each figures.

As the observation time is increased the diffusion coefficients for peak 1 (the slowest oil diffusion peak), within uncertainties, are approximately constant, i.e., $D$ ranges from $4 \times$ $10^{-14}$ to $1 \times 10^{-13} \mathrm{~m}^{2} \mathrm{~s}^{-1}$, indicating that we are measuring some kind of unrestricted diffusion. The product of $D \cdot \Delta$ increases for all values of $\Delta$, confirming that unrestricted diffusion is probed. For peak 2, the diffusion coefficient continuously decreases with incrementing observation time, varying within the range of $3.2 \times 10^{-13}$ and $6 \times 10^{-12} \mathrm{~m}^{2} \mathrm{~s}^{-1}$. The product of $D \cdot \Delta$ is seen to be constant.

\subsubsection{Rheology}

The form of the oscillatory response of the tetradecane system on increasing strain amplitude is very similar to that of the soybean oil and palm oil systems. As is shown in Fig. 8.8, the viscoelastic properties of the tetradecane system were measured as a linear region separated from the non-linear region at a value of strain amplitude around $10 \% . G^{\prime}$ and $G^{\prime \prime}$ in the linear region for soybean oil and tetradecane emulsions in domain I fall within an order of magnitude of each other, suggesting that despite the difference in Na-caseinate content the 
local structure of the tetradecane emulsions is very similar to that of the soybean oil emulsion in the same domain for emulsions with identical volume fractions.
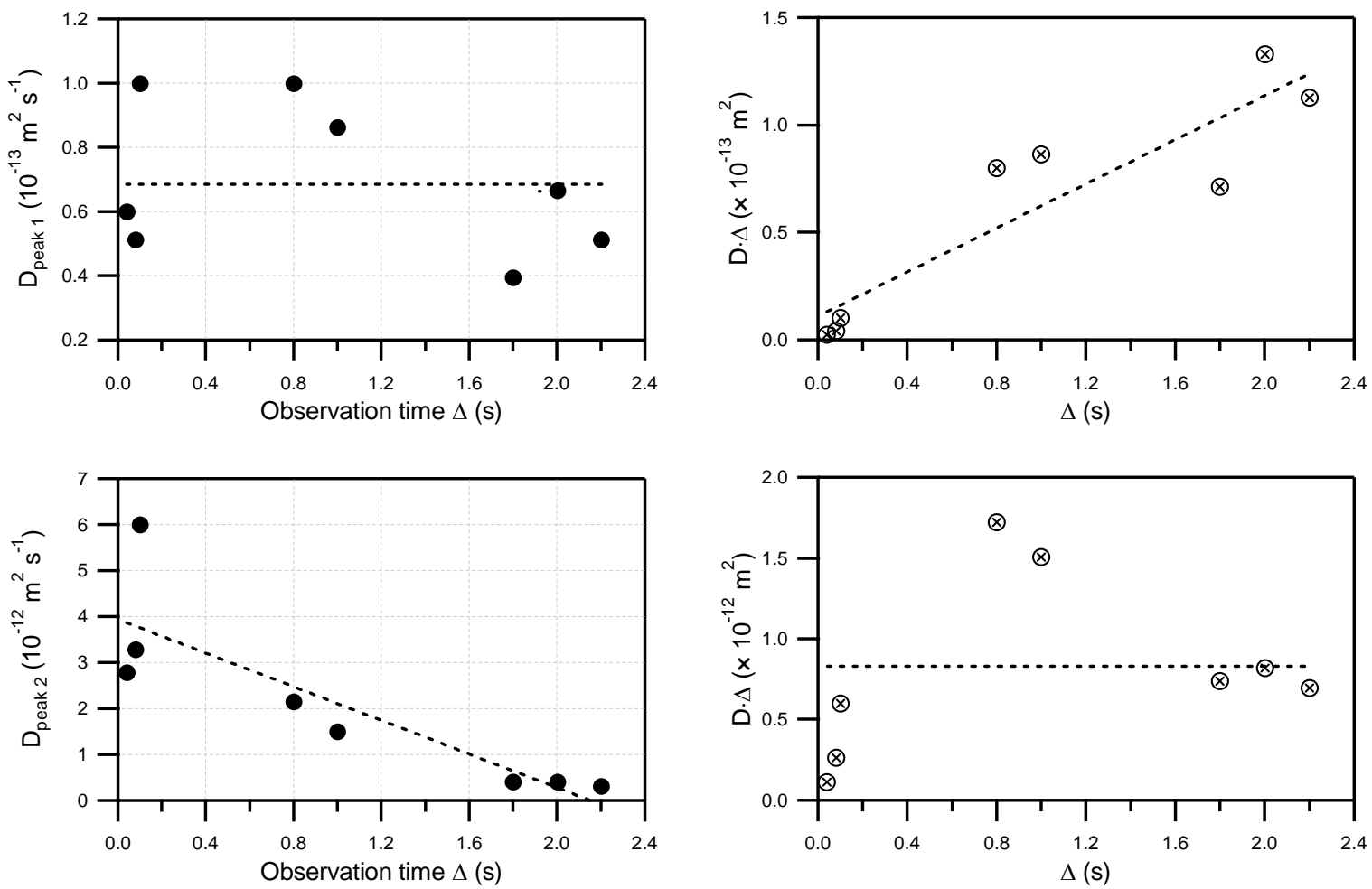

Figure 8.7: Tetradecane diffusion coefficients as a function of increasing observation time, $\Delta$, for 50 wt $\%$ tetradecane $/ 2 \mathrm{wt} \% \mathrm{Na}$-caseinate $/ 48 \mathrm{wt} \%$ water emulsion obtained by performing an inverse Laplace transform. The two figures on the left hand side represents the diffusion coefficient of one of the two different oil diffusion peaks at its maximum with the corresponding product of $D \cdot \Delta$ on the right hand side.

The effect of incrementing tetradecane and Na-caseinate concentrations on the linear viscoelastic response of the emulsions are given in Fig. 8.9 and Fig. 8.10, respectively. In both figures, it is seen that $G^{\prime}$ always lies above $G^{\prime \prime}$ over the entire range of frequencies investigated with $G^{\prime}$ and $G^{\prime \prime}$ separated by about an order of magnitude. Both $G^{\prime}$ and $G^{\prime \prime}$ increase on increasing either tetradecane or Na-caseinate concentrations. This oil dependent viscoelastic response observed in the tetradecane system (Fig. 8.9) is absent in soybean oil (see Fig. 5.14) and palm oil emulsions (see Fig. 7.8). 


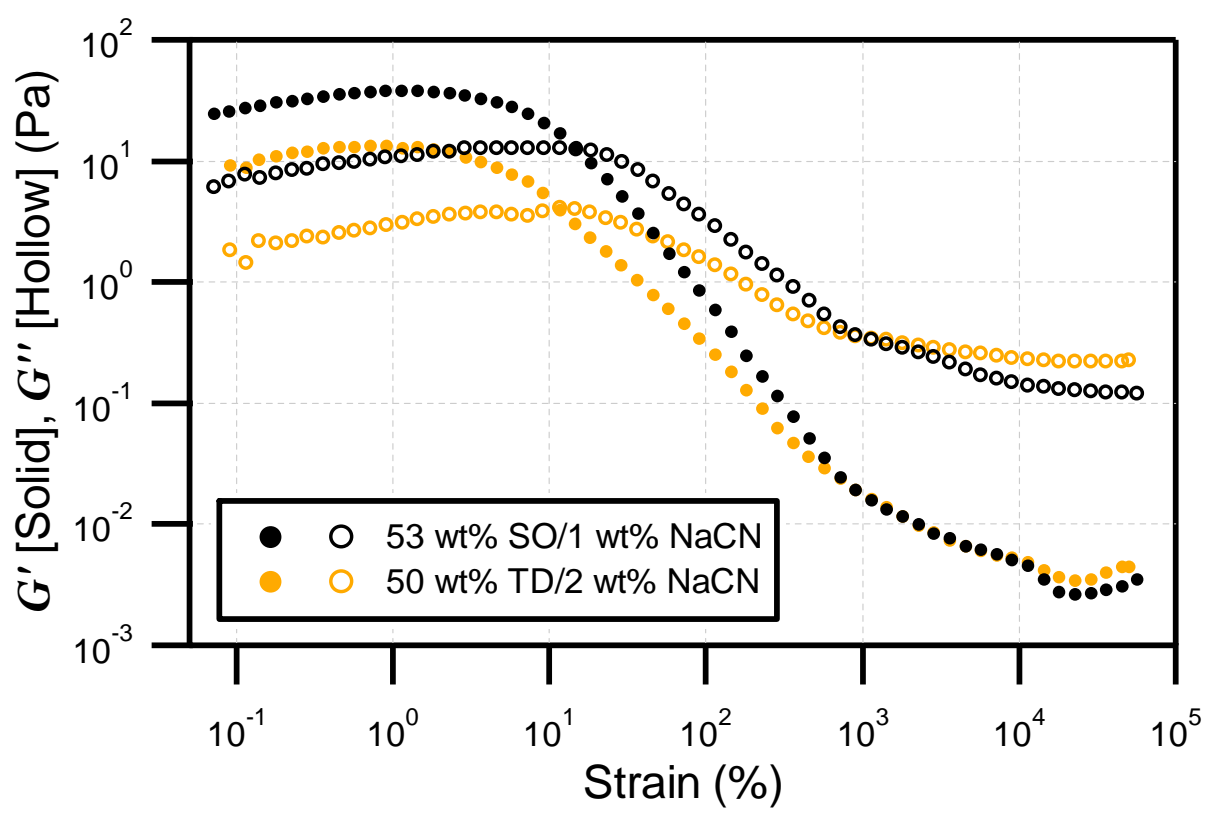

Figure 8.8: A representative log-log plot of the strain amplitude dependence of the storage and loss moduli, $G^{\prime}$ and $G^{\prime \prime}$ at $1 \mathrm{rad} \mathrm{s}^{-1}$, for freshly prepared $50 \mathrm{wt} \%$ tetradecane $/ 2 \mathrm{wt} \% \mathrm{Na}$-caseinate $/ 48 \mathrm{wt} \%$ water emulsion. We denote $\mathrm{SO}$ as soybean oil, TD as tetradecane and $\mathrm{NaCN}$ as Na-caseinate.
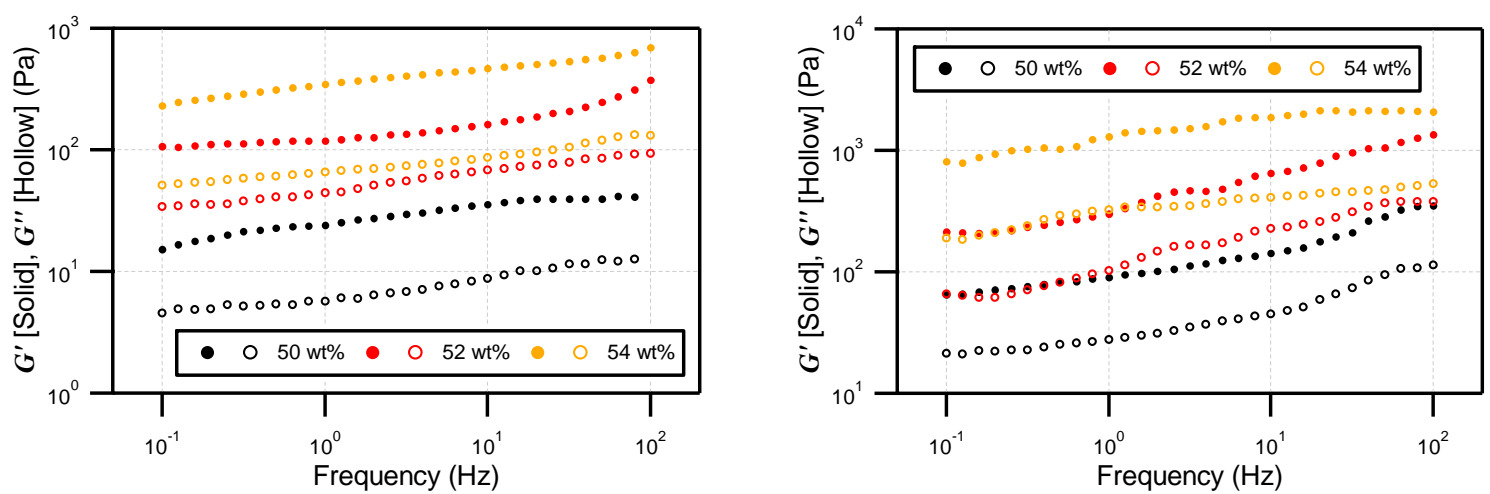

Figure 8.9: Log-log plot of the frequency dependence of $G^{\prime}$ and $G^{\prime \prime}$ at a strain amplitude of $2 \%$, for freshly prepared tetradecane/1 (left), $2 \mathrm{wt} \%$ (right) Na-caseinate/water emulsions.

In Fig. 8.11 is shown the steady state flow curves for $55 \mathrm{wt} \%$ tetradecane/3, 4 and 5 $w t \%$ Na-caseinate/water emulsions at different shearing times. The four rheological responses, as found for the soybean oil and palm oil systems (see Fig. 6.1 for identification) are also observed in the tetradecane system despite the macroscopic stability and microstructure variation of the emulsions in domains I and II being probed. On increasing Na-caseinate concentration, the stress response of the system increases. $\dot{\gamma}_{1}$ and $\dot{\gamma}_{2}$ values span approximately two orders of magnitude, i.e., from $\sim 0.03$ to $2 \mathrm{~s}^{-1}$ (Table 8.1). No trend is seen upon varying $\mathrm{Na}$-caseinate concentration. 


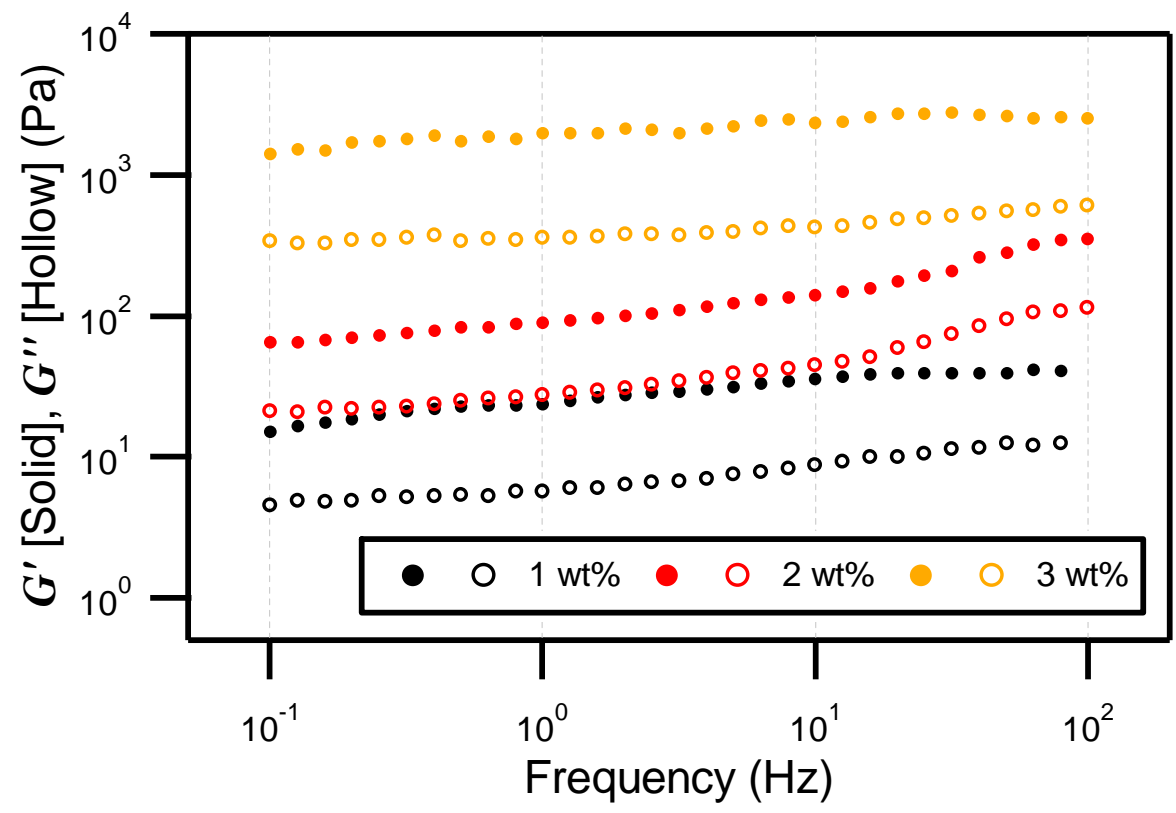

Figure 8.10: Log-log plot of the frequency dependence of $G^{\prime}$ and $G^{\prime \prime}$ at strain amplitude of $2 \%$ on increasing Nacaseinate concentration, for freshly prepared $50 \mathrm{wt} \%$ tetradecane/Na-caseinate/water emulsions.

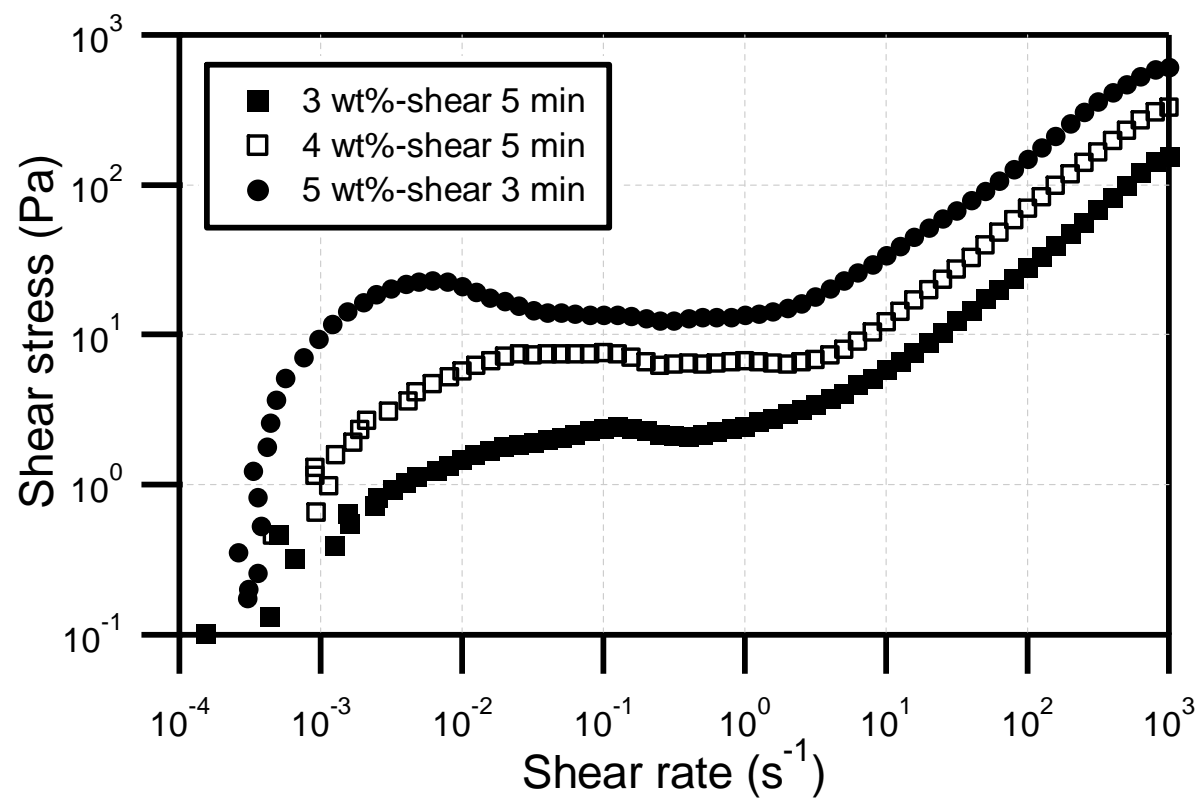

Figure 8.11: Stress vs. shear rate curves for $55 \mathrm{wt} \%$ tetradecane/3, 4 and $5 \mathrm{wt} \% \mathrm{Na}$-caseinate/water emulsions (bottom to top) during up-stress ramping: 1 min rest between each shear rate, from 0.0001 to $1000 \mathrm{~s}^{-1}$. Shearing times required to yield a steady state response varied with concentration and are given in the legend. $\dot{\gamma}_{1}$ and $\dot{\gamma}_{2}$ values (see definition in Fig. 6.1) span approximately two orders of magnitude on increasing Na-caseinate concentration. 
Table 8.1: $\dot{\gamma}_{1}$ and $\dot{\gamma}_{2}$ values extracted from steady state flow curves for 55 $\mathrm{wt} \%$ tetradecane emulsions plotted in Fig. 8.11

\begin{tabular}{c|ccc}
\hline Critical shear rate $\left(\mathrm{s}^{-1}\right)$ & \multicolumn{3}{|c}{ Na-caseinate concentration (wt\%) } \\
& 3 & 4 & 5 \\
\hline$\dot{\gamma}_{1}$ & 0.0631 & 0.0251 & 0.0398 \\
$\dot{\gamma}_{2}$ & 0.794 & 2.00 & 1.26 \\
\hline
\end{tabular}

\subsubsection{Cryo-SEM and Confocal Microscopy}

Cryo-SEM allows a single time image of the internal structure of a fluid sample to be obtained. Here the rapidly frozen fluid sample is fractured and then coated with platinum to ensure high resolution imaging is possible. The microstructures of a $52 \mathrm{wt} \%$ tetradecane/2 $\mathrm{wt} \%$ Na-caseinate $/ 46 \mathrm{wt} \%$ water emulsion acquired using secondary electrons and backscattered electrons are shown in Fig. 8.12. Fig. 8.12 (a) is a characteristic representation of the micrograph obtained using secondary electrons for the tetradecane system. While both water and oil domains are apparent with water presenting as smooth regions (e.g., see red circle bounded regions) the oil domains do not. The freezing rate is insufficient and crystallisation of the tetradecane occurs which occasionally ruptures the thin interfacial film causing the microstructure to be altered (clearly evident in Fig. 8.12 (a)). This hinders interpretation of the micrographs. This was not observed in the soybean oil and palm oil systems. As a result, it was not possible to determine the effect of incrementing Na-caseinate concentration on the microstructure of tetradecane emulsions.

The polydispersity of the oil droplet size could however be seen using the backscattered electron imaging mode. This is illustrated in Fig. 8.12 (b). A few very large oil droplets are seen mirroring the light scattering data. The droplet size distribution was also probed by staining emulsions with a hydrophobic fluorescing dye 4-(4methoxybenzylamino)-7-nitrobenzofurazan (green) and observing the emulsions with laser scanning confocal microscopy ${ }^{11}$. The dye was pre-dissolved in the tetradecane before homogenisation. From the light scattering data, the droplet size distribution of the dyed sample did not produce any significant deviation from that of fresh sample with no dye solubilised.

Considering the confocal image shown in Fig. 8.13, oil droplets are readily apparent with the dye confined within the oil droplets. The droplet size distribution corresponds well 
with static light scattering data. Network formation cannot at this stage be distinguished as data at one particular scanning plane over time would be required. However, what is evident from this data is that the average spacing between the oil droplets is much smaller than the average size of the oil droplets and as such restricted diffusion of the oil droplets would be expected to be probed in the PGSTE-NMR experiment. This was not the case. Note also that due to resolution limitations confocal microscopy is blind to oil droplets with sizes below $\sim 200 \mathrm{~nm}$.
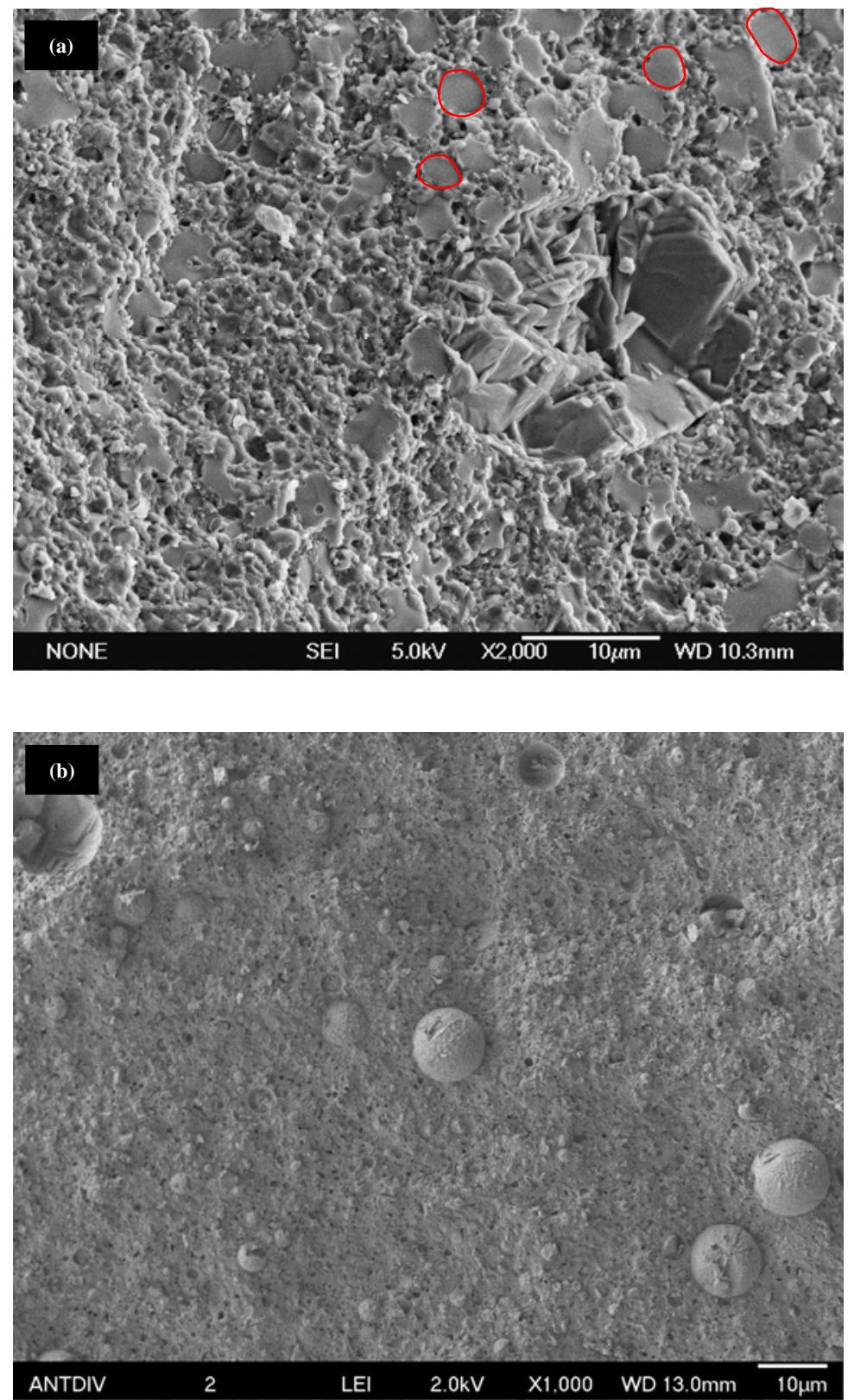

Figure 8.12: Cryo-SEM micrographs of (a) $2000 \times$ and (b) $1000 \times$ magnification for freshly prepared $52 \mathrm{wt} \%$ tetradecane $/ 2 \mathrm{wt} \%$ Na-caseinate $/ 46 \mathrm{wt} \%$ water emulsion obtained using (a): secondary electrons and (b): backscattered electrons, respectively. 


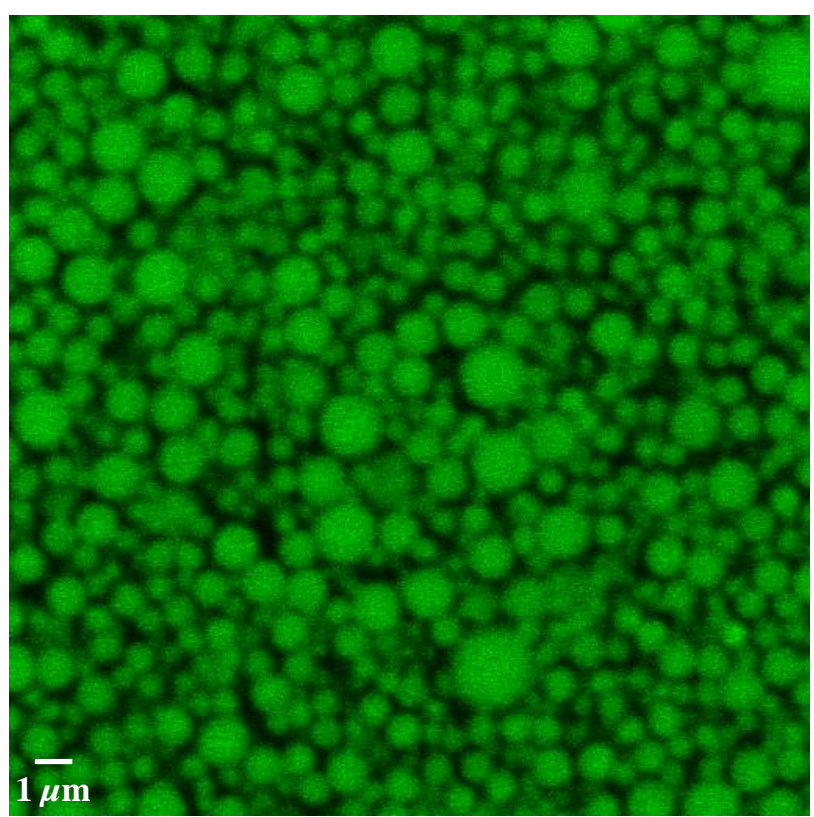

Figure 8.13: $2 \mathrm{D}(512 \times 512)$ confocal snapshot $(100 \times$ objective $)$ of freshly prepared $52 \mathrm{wt} \%$ tetradecane $/ 2 \mathrm{wt} \%$ Na-caseinate/46 wt\% water emulsion labelled with 4-(4-methoxybenzylamino)-7-nitrobenzofurazan (green).

\subsection{Discussion}

\subsubsection{Laser Diffraction Particle Sizing}

In order to compare the droplet size distribution of tetradecane emulsions with the other two oil systems, the oil droplet size was extracted at the trough between the lower and higher droplet modes and at the higher droplet mode as a function of oil concentration for the three different oil systems. The lower droplet mode consists of a mixture of small oil droplets and Na-caseinate aggregates while above this limit, consists purely of oil droplets and thereby gives a more direct comparison of the effect of changing oil. The data are summarised in Fig. 8.14 while in Fig. 8.15 are shown the droplet size distributions of freshly prepared soybean oil, palm oil and tetradecane $/ 2 \mathrm{wt} \% \mathrm{Na}$-caseinate/water emulsions for ease of comparison. Note that in Fig. 8.14 the extracted oil droplet size at the higher droplet mode for soybean oil and palm oil emulsions overlap with each other at the oil concentration of $52 \mathrm{wt} \%$.

As can be noticed in Fig. 8.14, within uncertainty, the oil droplet sizes obtained at the trough and from the higher droplet mode of the bimodal distribution for all three emulsion systems are identical. The major difference between the three oil systems is in the larger diameter tail of the higher droplet mode. The distribution is narrowest for soybean oil 
emulsions. For both palm oil and tetradecane emulsions, large oil droplets commonly form, these alter the number of oil droplets in the distribution and the interactions between the oil droplets and the average packing of the oil droplets. These changes are seen to manifest themselves in the rheological response of the emulsions and their specific microstructure.

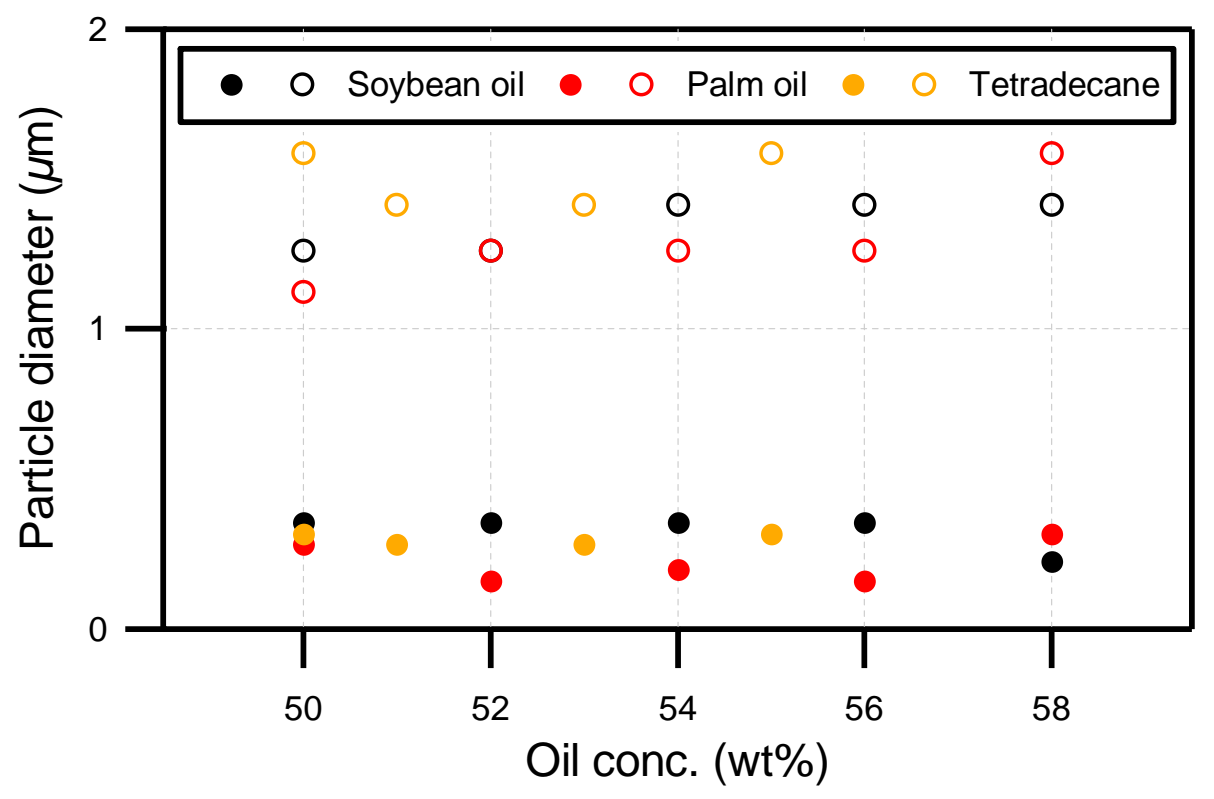

Figure 8.14: Extracted oil droplet size at the trough between the lower and higher droplet modes (solid circles) and at the higher droplet mode (open circles) as a function of increasing oil concentration for freshly prepared soybean oil, palm oil and tetradecane $/ 2 \mathrm{wt} \% \mathrm{Na}$-caseinate/water emulsions.

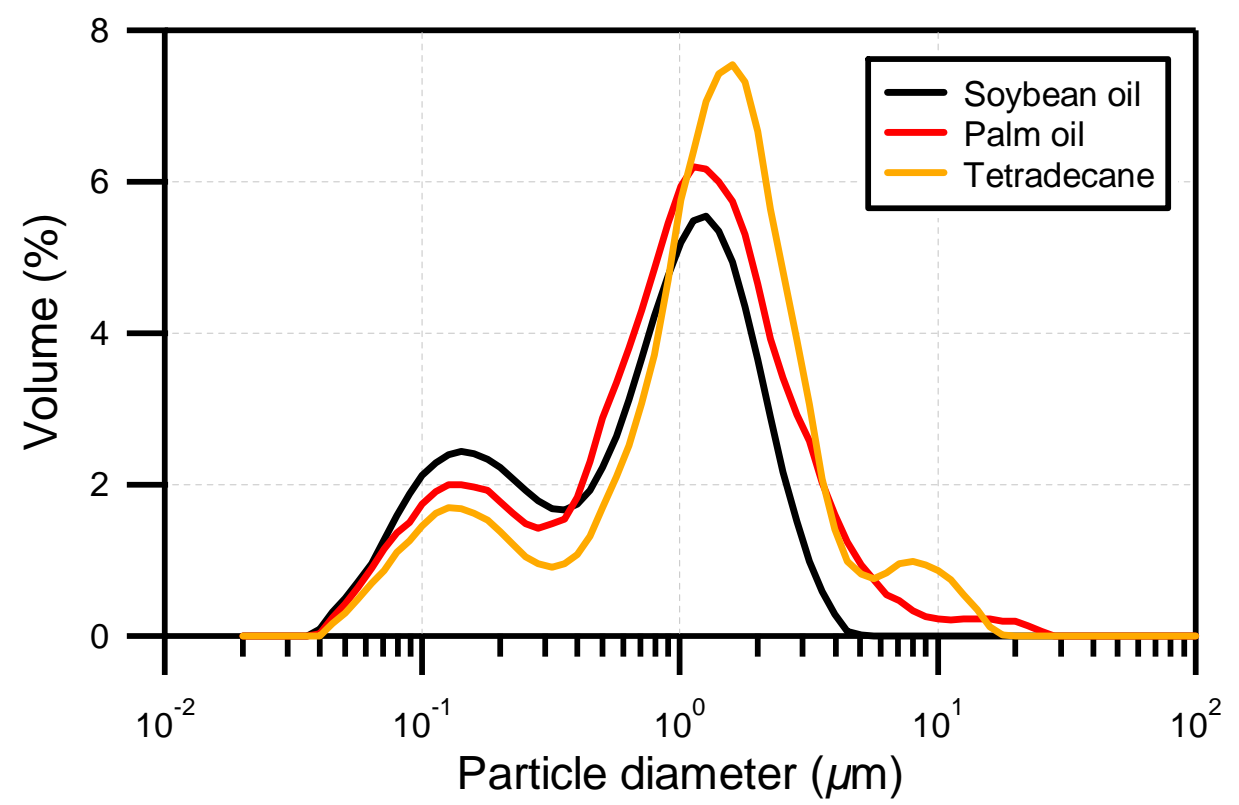

Figure 8.15: Droplet size distributions for freshly prepared $50 \mathrm{wt} \%$ soybean oil, palm oil and tetradecane $/ 2 \mathrm{wt} \%$ Na-caseinate $/ 48$ wt $\%$ water emulsions. 


\subsubsection{PGSTE-NMR}

As emulsions are dynamic systems, diffusion within oil droplets may occur in addition to the self-diffusion of the discrete ${ }^{12,13}$ and continuous phases over a certain time scale. This convolution is further complicated by the inherent polydisperse nature of the emulsions. By performing PGSTE-NMR ${ }^{14-16}$ the dynamic motion of water and the oil droplets can be both monitored and quantitatively determined. Water molecules are able to reside in three different domains of the system, i.e., the oil domain, the water domain and the interfacial domain. Since tetradecane is totally water-insoluble, only the diffusion of water molecules in the water and the interfacial domains is being measured on an ensemble average basis.

From our diffusion measurements of water, the straight decrease in diffusion coefficient on increasing tetradecane (Fig. 8.3) concentration and for those observed in soybean oil (see Fig. 5.5) and palm oil (see Fig. 7.3) systems at all values of $\Delta$ investigated hints the increasing tortuosity in the travelling pathway of the water molecules driven by the increasing number of oil droplets. Indeed, the concept of tortuosity of molecules has been widely utilised in porous media to probe the pore size, structure and connectivity through the applications of PGSTE-NMR theory ${ }^{17}$ and measurements ${ }^{18}$.

In Fig. 8.16 are shown the water diffusion coefficients at three different oil concentrations in wt\%, extracted from Fig. 5.5, Fig. 7.3 and Fig. 8.3 for freshly prepared soybean oil, palm oil and tetradecane $/ 2 \mathrm{wt} \%$ Na-caseinate/water emulsions, respectively. Switching from soybean oil to palm oil to tetradecane as the dispersed phase in emulsions containing $2 \mathrm{wt} \%$ Na-caseinate, the diffusion coefficient of water decreases. That is, for all three oil concentrations, the value of the water diffusion coefficients for soybean oil and tetradecane emulsions is the highest and the lowest, respectively. This reveals that the tortuosity of water molecules in the soybean oil emulsions containing $2 \mathrm{wt} \% \mathrm{Na}$-caseinate is lower than those in the palm oil and tetradecane emulsions. Through using the $\mathrm{wt} \% \mathrm{basis}$ it would appear that the movement of water molecules in the tetradecane system is affected the most. This suggests an enhancement of the droplet-droplet, water-droplet and/or unbound protein molecules occur in the tetradecane emulsions leading to a more apparent reduction in the diffusion of water. However, given the density difference between the oils (soybean oil, palm oil and tetradecane: $0.850,0.866$ and $0.767 \mathrm{~g} \mathrm{~cm}^{-3}$, respectively) we reconsider the data on a volume fraction basis. This provides different clues; all of which reveal that the interaction between the oil ${ }^{19,20}$ and the emulsifier ${ }^{21,22}$ which dictates curvature and interfacial 
film structure ${ }^{23-26}$ and therefore influences the diffusion of water, defines the stability of emulsions.

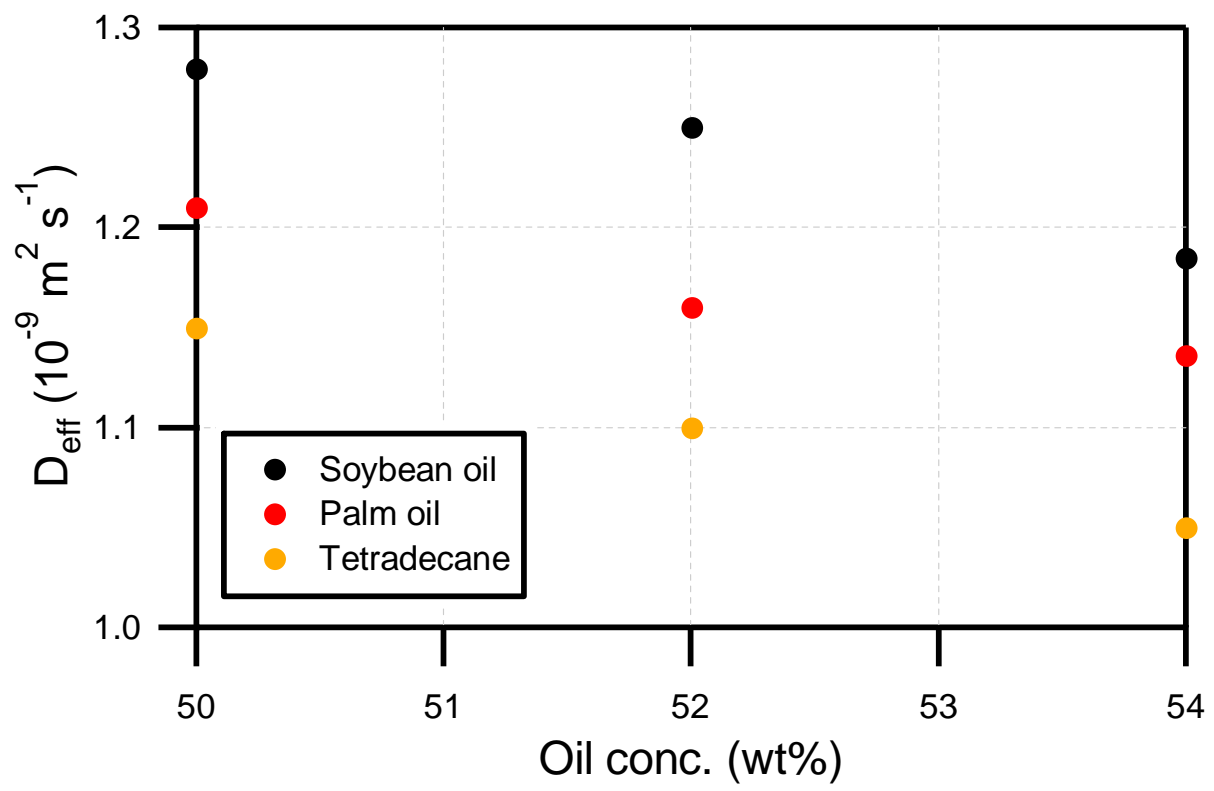

Figure 8.16: Diffusion coefficient of water as a function of increasing oil concentration in wt $\%$, extracted from Fig. 5.5, Fig. 7.3 and Fig. 8.3 for freshly prepared soybean oil, palm oil and tetradecane/2 wt $\%$ Nacaseinate/water emulsions, respectively.

Consider again the $2 \mathrm{wt} \%$ Na-caseinate water diffusion coefficient data (Fig. 8.17 right). On a volume basis the water diffusion coefficients in palm oil and tetradecane emulsions now fall on a continuum, while those in the soybean oil system remain faster. We note however that at $2 \mathrm{wt} \% \mathrm{Na}$-caseinate while both the palm oil and tetradecane emulsions are in domain I, those in the soybean oil system are in domain II. In domain I of the palm oil system, bridging flocculation is evident whereas in domain II of the soybean oil system individual oil droplets exist in a water continuum. This indicates that at $2 \mathrm{wt} \% \mathrm{Na}$-caseinate the microstructure of tetradecane emulsions correlates most directly with that of palm oil emulsions and bridging flocculation dominates the system. To confirm this, the water diffusion coefficients obtained for soybean oil emulsions containing $1 \mathrm{wt} \% \mathrm{Na}$-caseinate (domain I) are compared with that of tetradecane emulsion containing $1 \mathrm{wt} \% \mathrm{Na}$-caseinate (domain I), Fig. 8.17 left. While the match is not as good as for the $2 \mathrm{wt} \%$ Na-caseinate data we again find that within experimental uncertainty the two represent continuum data. This strengthens the argument that emulsions in domain I for all three oil systems have the same microstructure and are stabilised by bridging flocculation. 

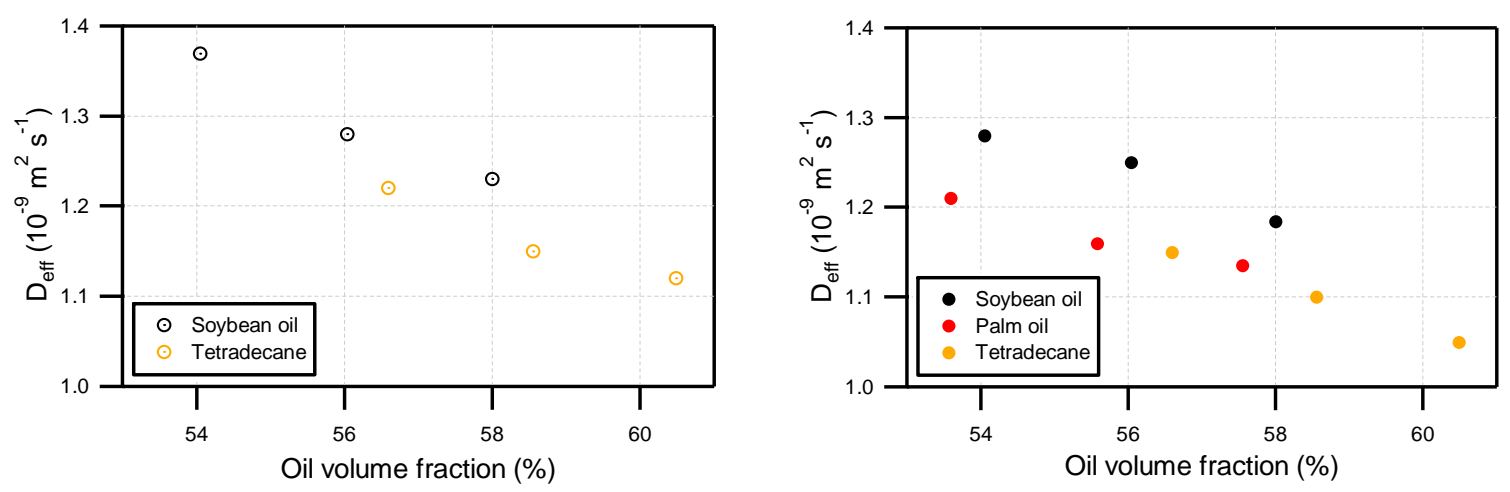

Figure 8.17: Diffusion coefficient of water as a function of increasing oil concentration in vol\%, extracted from Fig. 5.5, Fig. 7.3 and Fig. 8.3 for freshly prepared soybean oil, palm oil and tetradecane/left: 1 and right: $2 \mathrm{wt} \%$ Na-caseinate/water emulsions, respectively.

With regard to the use of PGSTE-NMR to probe tetradecane-Na-caseinate interactions, no investigations have dealt with this topic.

For the case of oil diffusion measurements, seemingly the tetradecane system is less complicated as compared with the soybean oil and palm oil systems, since only two, as opposed to four, diffusion peaks are evident. Before proceeding to the analysis of the diffusion NMR data of oil droplets and molecules based on its dependence on the observation time, $\Delta^{27}$, we first note that the unrestricted diffusion coefficient of tetradecane is $5.3 \times 10^{-10}$ $\mathrm{m}^{2} \mathrm{~s}^{-1}$. This fastest oil diffusion does not contribute to any of the measured oil diffusion peaks for the values of $\Delta$ used. We will explain the observed diffusion coefficient distribution using the $50 \mathrm{wt} \%$ tetradecane $/ 2 \mathrm{wt} \%$ Na-caseinate $/ 48 \mathrm{wt} \%$ water emulsion measured at $\Delta=100 \mathrm{~ms}$ as an example (see Fig. 8.6).

Based on the $D$ vs. $\Delta$ plot for peak 1 in Fig. 8.7, the measured diffusion coefficients remain approximately constant on varying $\Delta$. Moreover, the product of $D \cdot \Delta$ increased with increasing observation time. Peak 1 in Fig. 8.6 thus corresponds to unrestricted diffusion of the oil droplets. Using equation 3.1, the calculated mean square displacements for $\Delta=40$ to $2200 \mathrm{~ms}$ range from 0.07 to $0.52 \mu \mathrm{m}$ indicating that unrestricted diffusion of the oil droplets in the lower droplet mode of the bimodal droplet size distribution (Fig. 8.1) contributes to the measured diffusion coefficient for peak 1 in Fig. 8.6. This type of diffusion is not removed upon dilution, which correlates directly with the minimal shift of peak 1 upon dilution (Fig. 8.6). The slight shift in this peak upon dilution is most likely due to the removal of a minor contribution from restricted diffusion of larger oil droplets in the distribution.

For peak $2, D \cdot \Delta$ vs. $\Delta$ and $D$ vs. $\Delta$ plots exhibit a constant (when considering uncertainties) and decreasing trends, respectively, thus revealing restricted diffusion being the 
major contributor. Upon dilution (Fig. 8.6), peak 2 does not disappear, hence, it is not due to restricted diffusion of oil droplets but rather restricted diffusion of the oil molecules in the oil droplets. By inserting the measured diffusion coefficient for peak 2 in Fig. 8.6, i.e., $6 \times 10^{-12}$ $\mathrm{m}^{2} \mathrm{~s}^{-1}$ and the $\Delta$ used, i.e., $100 \mathrm{~ms}$ into equation 5.6, this corresponds to restricted diffusion of the oil molecules in the $3.5 \mu \mathrm{m}$ oil droplets, i.e., near the larger diameter tail of the higher droplet mode in Fig. 8.1.

During initial passthroughs of homogenisation, it is visibly evident that shear-induced flocculation occurs. Large flocs exist and emulsions are strongly resistant to flow. The occurrence of this phenomenon is primarily due to weak penetration of tetradecane into the interfacial domain which thereby enhances interdroplet interactions via bridging between the oil droplets. The average oil droplet spacing as estimated from the PGSTE-NMR data is less than $0.5 \mu \mathrm{m}$, this is supported by the confocal data. As such, it is expected that restricted diffusion of the larger oil droplets in the distribution should be probed, as was the case in the soybean oil and palm oil systems. However, this was not the case for the tetradecane system. This can be explained if we assume that weakly bound flocs are formed by the large oil droplets in the distribution. Hence, the large oil droplets diffuse collectively as the flocs and therefore their movement is slower than that detectable by the PGSTE-NMR technique, and as such we are now blind to these oil droplets. Owing to this, restricted diffusion between the discrete oil droplets is absent in the diffusion data. The formation of such flocs does not impede significantly the diffusion of the small oil droplets which still experience unrestricted diffusion (peak 1 in Fig. 8.6) though their diffusion coefficient is reduced compared to if they were just moving in an aqueous continuum due to the marked increase in the "continuous phase" viscosity. For oil droplets of $500 \mathrm{~nm}$ and smaller, a maximum diffusion coefficient of $\sim 1 \times 10^{-12} \mathrm{~m}^{2} \mathrm{~s}^{-1}$ is expected if the continuous phase is water having a viscosity of $0.89 \mathrm{mPa}$ s. However, the measured unrestricted diffusion is $\sim 6 \times 10^{-14} \mathrm{~m}^{2} \mathrm{~s}^{-1}$. Thus, the diffusion of the smaller oil droplets while being unrestricted over the range of $\Delta$ investigated are obstructed and travel through a higher viscosity medium resulting in a reduced average diffusion coefficient.

\subsubsection{Rheology}

The increasing function of both $G^{\prime}$ and $G^{\prime \prime}$ on increasing tetradecane and Na-caseinate concentrations as shown in Fig. 8.9 and Fig. 8.10 is the first direct evidence of an oil 
concentration effect. These data imply that the local structure of the tetradecane emulsions studied is dominated and defined by both tetradecane and Na-caseinate concentrations. Since the local structure is probed, variation in the interfacial domain accounts for the observed deviations of the oscillatory data of the soybean oil and palm oil systems. Insolubility of tetradecane causes reduced penetration at the interfacial domain. As such, in order to achieve a homogeneous emulsion the structural form and assembly of Na-caseinate at the interface with tetradecane as the dispersed phase is more finely manipulated compared to the much higher tolerance of oil at the interfacial domain in the soybean oil and palm oil systems. That is, upon increasing tetradecane concentration the interfacial domain is modified to a greater extent in order to produce stable emulsions. These variations in local structure manifest themselves as an increment in $G^{\prime}$ and $G^{\prime \prime}$. Nonetheless, $G^{\prime}$ (and also $G^{\prime \prime}$ ) remained approximately constant over the applied frequency range for all emulsions studied indicating the microstructure has not been altered in situ.

In the literature, the oscillatory rheological response of Na-caseinate stabilised oil-inwater emulsions has been reported by Dickinson et al. ${ }^{28}$. Their work focussed on the dilute region, i.e., $<45$ vol\% tetradecane. A high stress pre-shear was incorporated in the shearing protocol followed by a time dependent oscillatory measurement to explore the viscoelastic consequences of the developing particle network. The initial shearing at high stress breaks up the existing flocs and emulsions containing $2 \mathrm{wt} \%$ Na-caseinate appeared to be predominantly viscous $\left(G^{\prime \prime}>G^{\prime}\right)$ with $G^{\prime}$ and $G^{\prime \prime}$ being constant over the whole course of the experiment $(8 \mathrm{hrs})$. These data support our supposition that tetradecane emulsions are inherently comprised of flocs resulting in an elastic material. No pre-shear treatment was applied and our emulsions are predominantly solid-like $\left(G^{\prime}>G^{\prime \prime}\right)$. On the other hand, an initial drop followed by a steady increase in both $G^{\prime}$ and $G^{\prime \prime}$ with $G^{\prime \prime}>G^{\prime}$ and finally a cross over after $\sim 3 \times 10^{4} \mathrm{~s}$ corresponding to a gel-type network structures were observed for emulsion containing $6 \mathrm{wt} \% \mathrm{Na}$-caseinate ${ }^{28}$. In fact, this coincides with our findings where network formation has been shown to occur in Na-caseinate dispersions for Na-caseinate concentrations as low as $6 \mathrm{wt} \%$ (see Chapter 3). A sufficiently high protein concentration and high dispersed phase volume fraction are major factors affecting the formation of a threedimensional network structure. If the volume fraction of dispersed phase is not sufficiently high, the network will collapse under its own weight ${ }^{29}$. Despite the weak penetration of tetradecane at the interfacial domain, the volume fraction of tetradecane in our emulsions is much higher ( $>56 \mathrm{vol} \%$ ) leading to the formation of a floc network, this coupled with the 
existence of $\mathrm{Na}$-caseinate aggregates and individual protein in the continuous phase promotes the protein network and thus resulting in an elastic response.

\subsection{Conclusions}

All emulsions studied using tetradecane as the dispersed phase are of the oil-in-water type and have similar droplet size distributions. Extracted values from the trough and the higher droplet mode of the droplet size distributions show that the oil droplet sizes for tetradecane emulsions are very similar to those of soybean oil and palm oil emulsions.

PGSTE-NMR results show that the diffusion coefficient of water is a decreasing function on increasing both tetradecane and Na-caseinate concentrations indicating the travelling pathway of water molecules has been restricted. When volume fraction is considered, the data are consistent with the microstructure of tetradecane emulsions being similar to those of soybean oil and palm oil emulsions in domain I. Enhanced interdroplet interaction aided by the proteins accounts for the decrease in the values of the measured diffusion coefficients for tetradecane emulsions.

From the diffusion measurements of tetradecane, a seemingly less complicated system exists where only two oil diffusion peaks corresponding to different types of diffusion on increasing $\Delta$ were observed, as compared to four for soybean oil and palm oil systems. The absence of restricted diffusion of individual oil droplets in the diffusion data is speculatively assigned to the formation of large floc structures.

Comparing to soybean oil and palm oil emulsions, for the first time, the linear viscoelastic measurements show that $G^{\prime}$ and $G^{\prime \prime}$ both increased together on increasing tetradecane concentration. The weak penetration of tetradecane at the interfacial domain and the self-assembly state of $\mathrm{Na}$-caseinate results in an increasingly elastic response. 


\subsection{References}

1. M. Clausse, J. Heil, J. Peyrelasse and C. Boned, J. Colloid Interface Sci. 87, 584-586 (1982).

2. S. Y. Shiao, V. Chhabra, A. Patist, M. L. Free, P. D. T. Huibers, A. Gregory, S. Patel and D. O. Shah, Adv. Colloid Interface Sci. 74, 1-29 (1998).

3. F. Ysamberttb, R. Antona and J.-L. Salager, Colloids Surfs. A 125, 131-136 (1997).

4. L. T. Lee, D. Langevin, J. Meunier, K. Wonk and B. Cabane, Prog. Colloid Polym. Sci. 81, 209-214 (1990).

5. M. Gradzielski, D. Langevin and B. Farago, Phys. Rev. E 53, 3900-3919 (1996).

6. B. P. Binks, H. Kellay and J. Meunier, Europhys. Lett. 16, 53-58 (1991).

7. S. Chaieb, B. P. Binks and J. Meunier, J. Phys. II 5, 1289-1296 (1995).

8. M. Nydén and O. Söderman, Langmuir 11, 1537-1545 (1995).

9. E. Dickinson, in Food structure-its creation and evaluation, edited by J. M. V. Blanshard and J. R. Mitchell (Butterworths, London, 1988), pp. 41.

10. S. W. Provencher, Comput. Phys. Commun., 229-242 (1982).

11. D. Semwogerere and E. R. Weeks, in Encyclopedia of biomaterials and biomedical engineering, edited by G. E. Wnek and G. L. Bowlin (Dekker, New York, 2004).

12. T. Garasanin, T. Cosgrove, L. Marteaux, A. Kretschmer, A. Goodwin and K. Zick, Langmuir 18, 10298-10304 (2002).

13. K. G. Hollingworth, A. J. Sederman, C. Buckley, L. F. Gladden and M. L. Johns, J. Colloid Interface Sci. 274, 244-250 (2004).

14. Y. Cohen, L. Avram and L. Frish, Angew. Chem. 44, 520-554 (2005).

15. E. O. Stejskal and J. E. Tanner, J. Chem. Phys. 42, 288-292 (1965).

16. J. E. Tanner, J. Chem. Phys. 52, 2523-2526 (1970).

17. P. P. Mitra, P. N. Sen and L. M. Schwartz, Phys. Rev. B 47, 8565-8574 (1993).

18. R. W. Mair, G. P. Wong, D. Hoffmann, M. D. Hürlimann, S. Patz, L. M. Schwartz and R. L. Walsworth, Phys. Rev. Lett. 83, 3324-3327 (1999).

19. G. A. van Aken, Colloids Surfs. A 213, 209-219 (2003).

20. V. Rampon, C. Brossard, N. Mouhous-Riou, B. Bousseau, G. Llamas and C. Genot, Adv. Colloid Interface Sci. 108-109, 87-94 (2004). 
21. V. K. Bansal, D. O. Shah and J. P. O’Connell, J. Colloid Interface Sci. 75, 462-475 (1980).

22. R. Leung, D. O. Shah and J. P. O’Connell, J. Colloid Interface Sci. 111, 286-287 (1986).

23. S. J. Chen, D. F. Evans and B. W. Ninham, J. Phys. Chem. 88, 1631-1634 (1984).

24. D. J. Mitchell and B. W. Ninham, J. Chem. Soc., Faraday Trans. 77, 601-629 (1981).

25. B. W. Ninham, D. F. Evans and S. J. Chen, J. Phys. Chem. 88, 5855-5857 (1984).

26. D. F. Evans, D. J. Mitchell and B. W. Ninham, J. Phys. Chem. 90, 2817-2825 (1986).

27. E.-H. Liu, H. Egger and K. M. McGrath, J. Phys. Chem. B 109, 13332-13341 (2005).

28. E. Dickinson and M. Golding, J. Colloid Interface Sci. 191, 166-176 (1997).

29. H. Luyten, M. Jonkman, W. Kloek and T. van Vliet, in Food colloids and polymers: stability and mechanical properties, edited by E. Dickinson and P. Walstra (Royal Society of Chemistry, Cambridge, 1993), pp. 224. 


\section{Chapter 9}

\section{Conclusions and Future Work}

\subsection{Conclusions}

In this thesis are presented studies on Na-caseinate dispersions and three emulsion systems using two different food grade oils: commercial soybean oil and commercial palm oil and one laboratory straight chain hydrocarbon: tetradecane with commercial Na-caseinate as the controlling agent.

In Na-caseinate dispersions, it was found that concentration effects define the interactions between casein aggregates. This was supported by the diffusion data where enhanced packing interaction induces formation of aggregates at low Na-caseinate concentration and causes a decrease in the protein diffusion coefficient. At sufficiently high protein concentration, a macroscopic gel is formed, the result of interaggregate assembly. Upon shearing at a constant shear rate, an irreversible gel formation occurs at Na-caseinate concentrations well below those required for microscopic gel formation. The gelation process occurs at significantly reduced times on increasing Na-caseinate concentration or shear rate. This gelled state is believed to be the result of protein self-assembly as opposed to interaggregate interactions. All samples, including the macroscopic gels respond locally as fluids.

From the perspective of emulsion technology, the above findings suggest that confining the casein proteins to a smaller volume, such as in the continuous phase of a concentrated emulsion or a high local protein concentration at the oil/water interface, enhances the protein interaction and the ability to form a three-dimensional gel in the continuous phase of the emulsion thereby having the potential to control the response of the emulsion. Furthermore, an irreversible gel system can be induced within the continuous phase by shearing for a long period of time. 
Oil-in-water emulsions form over nearly the entire range of oil concentrations investigated (50 to $70 \mathrm{wt} \%$ ) in all three systems except for those at the phase boundaries where a highly gelled system was able to be realised. These emulsions are polydisperse. All of the emulsions formed exhibit similar droplet size distributions upon preparation. The distribution is essentially bimodal. Thus, droplet size is not a control factor in defining emulsion stability. Although emulsions formed in all three systems appeared as visually homogeneous white suspensions, they are rather complex systems. The emulsion stability is not only a function of different oils but a function of $\mathrm{Na}$-caseinate concentration indicates that the macroscopic phase separation must depend, in addition to the concentration of the dispersed phase, on the specific nature of the interfacial film, droplet-droplet and oilemulsifier-water interactions; all of which can lead to modification of the interfacial domain and hence different degrees of destabilisation.

The phase spaces in the phase diagrams of the water soluble, high interfacial penetration oils; soybean oil and palm oil, are very rich and similar with emulsion microstructure being strongly influenced by the Na-caseinate content. On increasing Nacaseinate concentration bridging flocculation, optimal surface coverage, depletion flocculation and the formation of a three-dimensional network are evidenced in cryo-SEM micrographs, manipulating the stability of the emulsions. True gel formation in the kinetic phase space leading to the formation of stable emulsions is determined by the Na-caseinate concentration. The two gel states obtained are different. At high Na-caseinate concentration and at low oil content, it is initiated by the inherent nature of the protein self-assembly and enhanced interaggregate interaction. At low Na-caseinate concentration and at high oil content is induced by enhanced interdroplet interaction.

Reducing interfacial penetration with the introduction of tetradecane dramatically manipulates the interfacial domain and restricts the diversity of emulsions formed. The phase diagram is less extended and emulsion formation is found to be energy input sensitive as a result of the reduced in the extent of penetration of tetradecane to the interface and hence a less rigid oil/water interface. Enhanced flocculation occurs at high concentrations of Nacaseinate and/or oil. Dilution prior to oil diffusion measurement confirmed the formation of weakly bound flocs that was not observed in the soybean oil and palm oil systems. When the water diffusion data is considered on a volume basis rather than a weight basis, tetradecane emulsions studied have the same microstructure as the soybean oil and palm oil emulsions which are stabilised by bridging flocculation. 
The addition of oil to Na-caseinate dispersion which is locally fluid-like, to form an emulsion, caused the formation of viscoelastic layer due to protein adsorption at the interface leading to a locally solid-like emulsion. The microstructural change due to colloidal interactions between adsorbed protein layers on different droplets manifest itself in the smalldeformation rheology. Higher oil and Na-caseinate concentration promote the packing of the oil droplets and hence results in the enhancement of viscoelastic properties of the system. The slight increase in the storage and loss moduli on incrementing the frequency in all emulsions further support the formation of a gel-like structure and correlates well with the self-assembly nature of Na-caseinate to form a three-dimensional network within the continuous phase.

Shear banding which appears to be ubiquitous in complex soft matter systems was observed in the three emulsion systems irrespective of oil and Na-caseinate concentrations. This means that the differences in the aggregated state of the proteins, the differences in the structure of the interfacial domain and therefore the interdroplet interactions are not probed. Inducement of the shear-banded state is not due to the occurrence of the emulsion phase transitions but rather the competition and dominance of two underlying physical effects, i.e., restructuration and destruction which are functions of shear rate and shearing time and change the emulsion macrostructure but not microstructure. The overall flow curve evolution is thereby greatly influenced by the local and collective rearrangements leading to continual break up and renewal of long length scale aggregates of oil domains. Due to the complex nature of the interplay between shear effects and ageing, concentrated emulsions may be better classified as soft glassy material such as gels, foams, pastes and suspensions as compared to a traditional emulsion which are generally shear-thinning fluids.

\subsection{Future Work}

The thermodynamic phase diagrams of the three ternary systems investigated in this thesis have only been qualitatively determined. As such a quantitative study of these phase diagrams is worthwhile. On the other hand, the relationship between the microstructures and the interfacial domains has not been fully probed. An immediate extension to the work outlined in this thesis would be to carry out in situ measurement to explore the interfacial physics and properties of the Na-caseinate emulsifier in each of the three systems for each of 
the different emulsion microstructures. Further, the region in the phase diagrams where there is a switch from coarse emulsions to gels should be explored in addition to the determination of the factors that induce the change in the system. It would be interesting to study other oil systems and also how the characteristics of emulsions formed change on varying the chain length of the oil would aid in the understanding of controlling and manipulating the interfacial domain.

The amount and the way energy input is delivered during emulsion formulation have been found to be able to define and affect the emulsion properties and microstructures. A detailed investigation of this should be performed.

It is known that Ostwald ripening is a contributing process in the destabilisation of Nacaseinate emulsions. Additionally, the rate of Ostwald ripening depends on the solubility of the dispersed phase ${ }^{1-4}$. But, how does the rate of Ostwald ripening vary as a function of different oils? Is the rate of Ostwald ripening influenced by droplet size? To address these questions, an investigation of the relationship between the rate of Ostwald ripening and different types of oils should be undertaken.

Currently we do not have additional proof of the different rheological behaviours obtained with respect to the application of continuous shear in the three systems apart from the stress response for each peak hold which form a flow curve and velocity profiles from NMR velocimetry. The shear-banded state bounded by a shear-thinning region at lower shear rates, and Newtonian behaviour at higher shear rates requires further evidence. Other imaging techniques could be incorporated in conjunction with rheometry to obtain direct and concrete hints on the structure formed in the sheared region.

While this thesis focuses on the study of concentration to the physicochemical properties of Na-caseinate emulsions, three other important aspects, i.e., the $\mathrm{pH}$, ionic strength and temperature should be emphasised in future works to gain a better understanding of their effects on defining the interfacial domain and the aggregation of casein which thereby directs the microstructure and stability of concentrated emulsions.

This should finally lead to an investigation of the influence of calcium ions to caseinatebased concentrated emulsions. As is known, in bovine milk, $\alpha_{\mathrm{s} 1^{-}}, \alpha_{\mathrm{s} 2^{-}}$and $\beta$-casein are the three calcium-sensitive members ${ }^{5,6}$. The influence of calcium ions on the stability of caseinate emulsions was recently reported. Emulsions containing sufficient ionic calcium were found to aggregate rapidly under quiescent conditions ${ }^{7-10}$ leading to a strong cross-link via enhanced protein-protein interaction ${ }^{11-14}$ but when emulsions were placed in a shear field 
the aggregation was observed to be initially slow, becoming more rapid as the shearing time was increased ${ }^{15,16}$. The work from Dickinson et al. ${ }^{9}$, for example, showed that the addition of 5 or $8 \mathrm{mM} \mathrm{CaCl}_{2}$ enhanced greatly the stability of Na-caseinate emulsions (10 or $35 \mathrm{vol} \%$ oil). However, Na-caseinate emulsions (25 vol\% oil) containing at least $10 \mathrm{mM}$ ionic calcium added before or after emulsification were found to exhibit an increasing average droplet size with time and a developing bimodal droplet size distribution ${ }^{13}$. Under turbulent conditions of intense shearing, the emulsions displayed time dependent flocculation and coalescence.

The shear-thinning behaviour of the caseinate-stabilised emulsions is typical of systems undergoing flocculation. Radford et al. ${ }^{17}$ found that the addition of calcium ions prior to homogenisation leads to a pronounced reduction in viscosity and the onset of Newtonian flow in Na-caseinate-stabilised emulsion (30 vol\% oil). Addition of calcium ions reduces the submicelle number density via specific calcium-caseinate binding in the aqueous phase and increases the amount of caseinate associated with the oil/water interface (increased surface coverage).

In contrast to relatively dilute emulsions, little information is available on the details of influence of calcium ions on the adsorption behaviour of caseinate in concentrated emulsions. Useful insights will be able to be gained through the study of the effect of calcium ion as the fourth component to the soybean oil, palm oil or tetradecane/Na-caseinate/water emulsions. 


\subsection{References}

1. A. Kabalnov, J. Dispersion Sci. Technol. 22, 1-12 (2001).

2. A. S. Kabalnov, K. N. Makarov, A. V. Pertsov and E. D. Shchukin, J. Colloid Interface Sci. 138, 98-104 (1990).

3. P. Taylor, Adv. Colloid Interface Sci. 75, 107-163 (1998).

4. P. Taylor, Adv. Colloid Interface Sci. 106, 261-285 (2003).

5. D. G. Dalgleish and T. G. Parker, J. Dairy Res. 47, 113-122 (1980).

6. T. G. Parker and D. G. Dalgleish, J. Dairy Res. 48, 71-76 (1981).

7. M. Srinivasan, H. Singh and P. A. Munro, J. Agric. Food Chem. 44, 3807-3811 (1996).

8. E. Dickinson, J. A. Hunt and D. S. Horne, Food Hydrocolloids 6, 359-370 (1992).

9. $\quad$ E. Dickinson and M. Golding, Colloids Surfs. A 144, 167-177 (1998).

10. E. P. Schokker and D. G. Dalgleish, Colloids Surfs. A 145, 61-69 (1998).

11. J. A. Hunt, E. Dickinson and D. S. Horne, Colloids Surfs. A 71, 197-203 (1993).

12. E. Dickinson, R. H. Whyman and D. G. Dalgleish, in Food emulsions and foams, edited by E. Dickinson (Royal Society of Chemistry, London, 1987), pp. 40.

13. E. Dickinson and E. Davies, Colloids Surfs. B 12, 203-212 (1999).

14. O. D. Velev, B. E. Campbell and R. P. Borwankar, Langmuir 14, 4122-4130 (1998).

15. S. O. Agboola and D. G. Dalgleish, J. Food Sci. 60, 399-404 (1995).

16. J. Chen, E. Dickinson and G. Iveson, Food Structure 12, 135-146 (1993).

17. S. J. Radford, E. Dickinson and M. Golding, J. Colloid Interface Sci. 274, 673-686 (2004). 


\section{Appendix}

Aggregate size distribution for 2, 6, 14 and $18 \mathrm{wt} \%$ Na-caseinate dispersions, indicating aggregate formation across the size range of $\sim 40 \mathrm{~nm}$ to $\sim 1 \mu \mathrm{m}$ :

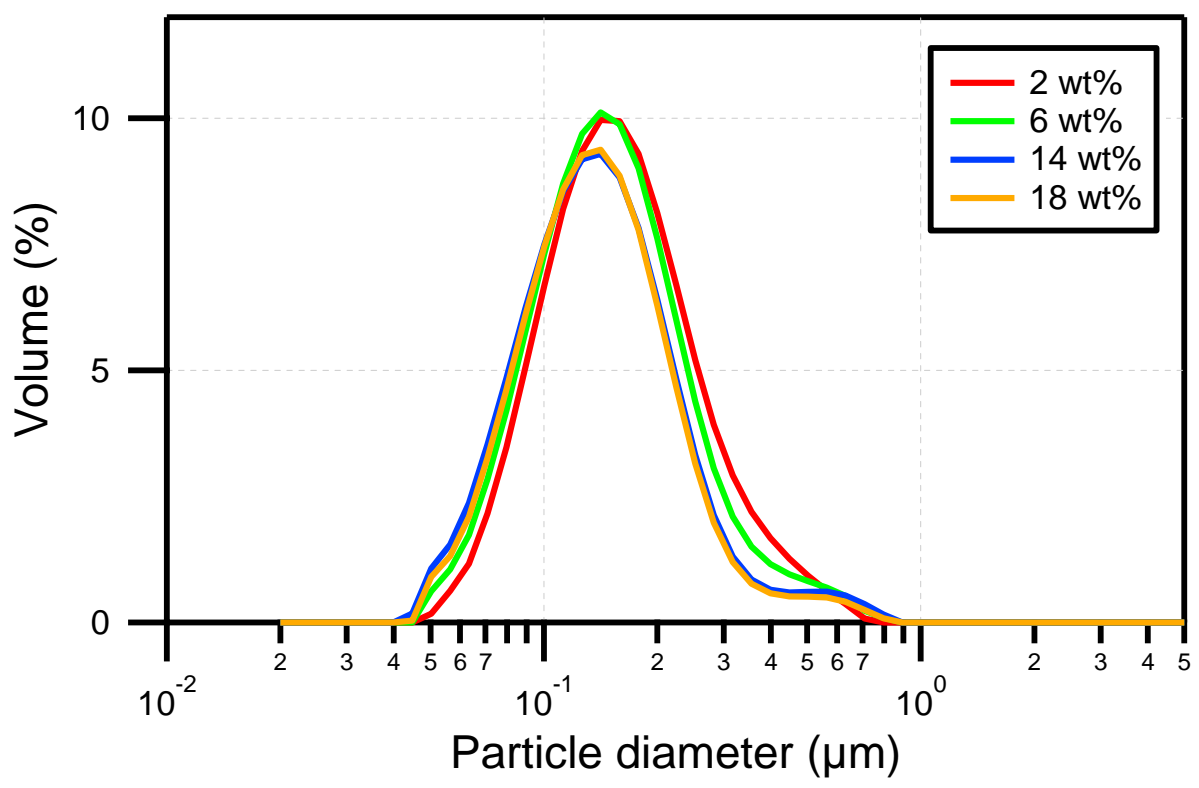

